\title{
BIOMECHANICS OF A PARASITIC WASP OVIPOSITOR _ Probing for answers
}

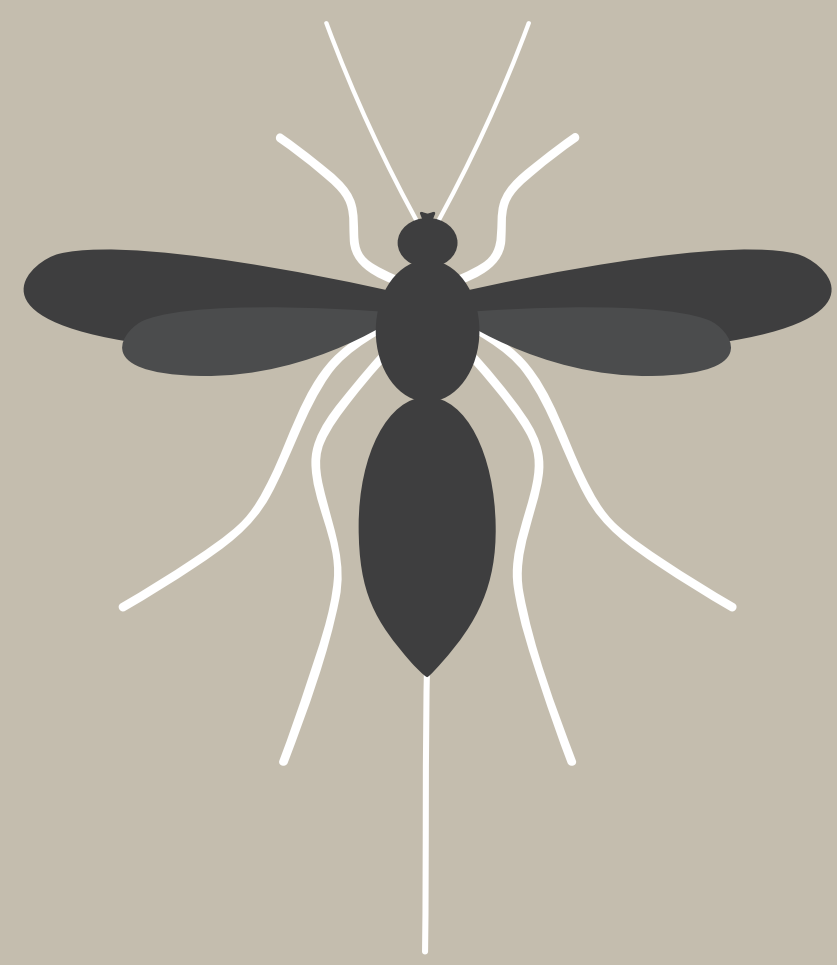

Uroš Cerkvenik 



\section{Biomechanics of a parasitic wasp ovipositor}

Probing for answers

Uroš Cerkvenik 


\section{Thesis committee}

\section{Promotor}

Prof. Dr J.L. van Leeuwen

Professor of Experimental Zoology

Wageningen University \& Research

\section{Co-promotor}

Dr S.W.S. Gussekloo

Assistant professor, Experimental Zoology

Wageningen University \& Research

\section{Other members}

Prof. Dr M. Dicke, Wageningen University \& Research

Prof. Dr P. Aerts, University of Antwerp, Belgium

Prof. (Em.) Dr E.J. Stamhuis, University of Groningen

Dr W. Federle, Cambridge University, UK

This research was conducted under the auspices of the Graduate School Wageningen Institute of Animal Sciences (WIAS) 


\title{
Biomechanics of a parasitic wasp ovipositor
}

\author{
Probing for answers
}

Uroš Cerkvenik

Thesis

submitted in fulfilment of the requirements for the degree of doctor at

Wageningen University

by the authority of the Rector Magnificus

Prof. Dr A.P.J. Mol,

in the presence of the

Thesis Committee appointed by the Academic Board

to be defended in public

on Tuesday 15 January 2019

at 4 p.m. in the Aula. 
Uroš Cerkvenik

Biomechanics of a parasitic wasp ovipositor: Probing for answers, 220 pages.

PhD thesis, Wageningen University, Wageningen, the Netherlands (2019)

With references, with summary in English, Slovenian, and Dutch

ISBN 978-94-6343-534-5

DOI $10.18174 / 462755$ 




\section{Table of Contents}

Chapter 1 General introduction 99

Chapter 2 Functional principles of steerable multi-element probes in insects $\quad 23$

Chapter 3 Mechanisms of ovipositor insertion and steering of a parasitic wasp

Chapter 4 Stiffness gradients facilitate ovipositor bending and spatial probing control in a parasitic wasp

Chapter 5 The ovipositor actuation mechanism of a parasitic wasp

Chapter 6 General discussion

Summaries

Summary

Povzetek (Slovenian)

Samenvatting (Dutch)

Acknowledgements

About the author

Biography

List of publications

Overview of completed training activities 

$\Upsilon_{\text {chapter }} 1$

General introduction 



\section{Introduction}

Many insects are capable of extracting or inserting liquids and eggs in different types of substrates. Female mosquitoes (family Culicidae) and true bugs (order Hemiptera) are specialised for the uptake of liquid food, ${ }^{1,2}$ whereas injecting eggs deep inside a substrate is often done by parasitic wasps (order Hymenoptera). ${ }^{3}$ These animals search for targets in diverse substrates ranging from viscoelastic vertebrate skin (mosquitoes and some hemipterans) ${ }^{4}$ to stiff plant tissues such as seeds, galls, or even wood (hemipterans and parasitic wasps) $)^{5-7}$. Entering a solid substrate and finding a target within is challenging and special mechanical, motor, and sensory adaptations evolved in insects that allow them to effectively probe in various substrates. In this thesis, I only briefly discuss the sensory aspect of probing, while investigating the mechanical and motor adaptations of probing in insects in depth.

Probing for resources seems to be advantageous as it evolved separately in disparate insect taxa. The probes of hemipterans and hymenopterans presumably made it possible to adapt to a wide range of substrates leading to a high diversification of these taxa. ${ }^{8-10}$ Both, hemipterans and hymenopterans are currently some of the most abundant insect orders and number around $180000^{11}$ and $145000^{12}$ species, respectively. Their probes can therefore be considered as key evolutionary innovations. $^{8}$

\section{Functional morphology of insect probes}

Insect probes are exoskeletal structures originating from different parts of the body. Mosquitoes and hemipterans use their mouthparts, ${ }^{13,14}$ while parasitic wasps probe with ovipositors, tubular structures originating from their abdomens. The ovipositors are used for targeted deposition of eggs in/on host larvae (Fig. 1A, B). ${ }^{15-17}$ Interestingly, probes across the mentioned taxa share a common structural feature-they consist out of multiple, longitudinally connected elements devoid of musculature. ${ }^{18-20}$ The number of probe elements differs across insect taxa. Six elements, called stylets, form the mouthparts of a female mosquito, ${ }^{18}$ aphid mouthparts contain four stylets, ${ }^{21}$ and parasitic wasp ovipositors generally consist out of three functional elements (Fig. 1C). ${ }^{10}$ The probes have a different developmental origin (i.e. mouthparts and abdominal structures), so it is clear why they are structurally different, although some of the variation might also reflect the different functional requirements across taxa.

The probes are used to explore different substrates and are used in different ways 

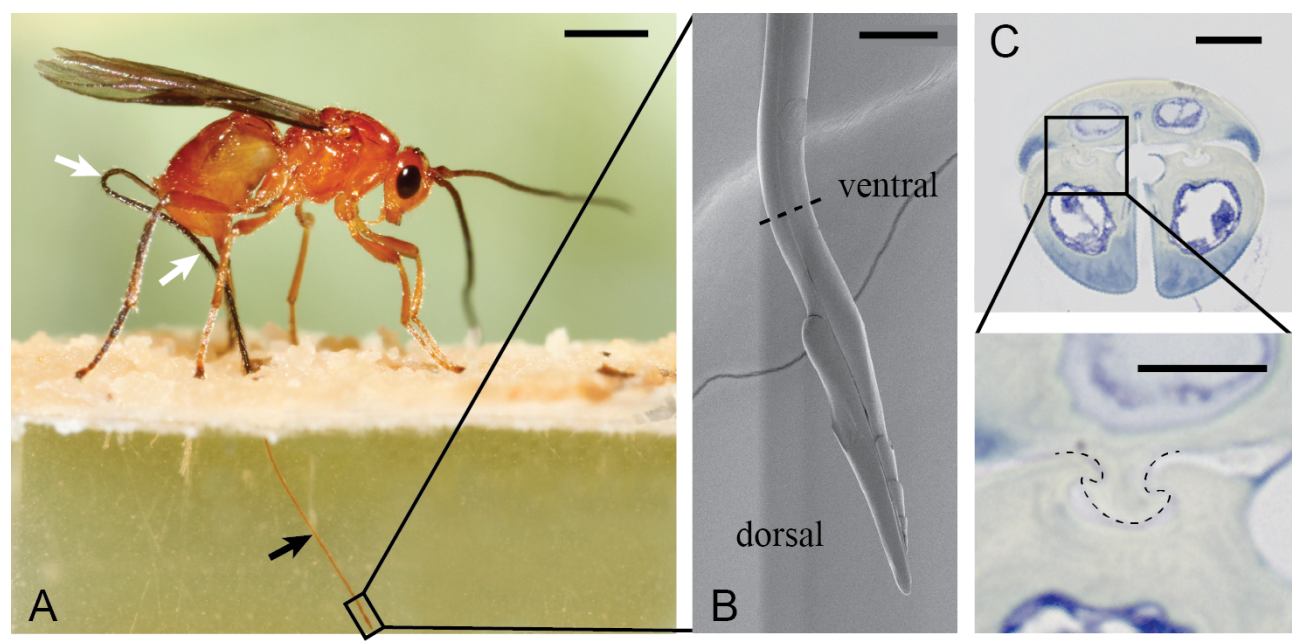

Fig. 1. A female parasitic wasp Diachasmimorpha lonigcaudata Ashmead (Braconidae) probing in the substrate. (A) The wasp is inserting its ovipositor (black arrow) inside a translucent gel ( $2 \%, E \approx 36 \mathrm{kPa})$. The ovipositor is outside the substrate supported by sheaths that peel away from the probe at its base (white arrows). (B) An SEM image of the morphologically distinct distal end (tip) of the ovipositor. The dorsal valve tip is enlarged, while the ventral valve tips bear smaller harpoon-like serrations. Proximal to the tip, the ovipositor is characteristically S-bend. The black, dashed line indicates the approximate location of the cross-section shown in C. (C) The ovipositor consists of three valves: a dorsal valve (top) and two ventral valves (bottom). The valves are interconnected with rail-like mechanism (square denotes the magnified region shown below) that allow for sliding but prevent valve separation. Scale bars: $1 \mathrm{~mm}$ (A), $100 \mu \mathrm{m}(\mathrm{B}), 10 \mu \mathrm{m}$ (C, top), and $5 \mu \mathrm{m}$ (C, bottom).

across insects. The substrate might have affected the material composition of the probes during evolution of probing insects. For example, fig wasps that probe in stiff fruits have more sclerotized (and therefore presumably stiffer) ovipositors than those probing in soft fruits. ${ }^{16}$ Additionally, the ovipositor stiffness in two fig wasp species, a pollinator and its parasitoid, has been measured. ${ }^{22}$ The ovipositor shafts of both species had similar Young's moduli, namely 0.92 GPa (pollinator) and $0.73 \mathrm{GPa}$ (parasitoid), but the tip of the parasitoid ovipositor was much stiffer $(1.42 \mathrm{GPa})$ than the shafts in either of the species. ${ }^{22}$ The stiffness of the ovipositor tip in the pollinator species was not reported. This indicates that insect probes are structurally and materially complex and could be adapted to the substrates.

An indication on how insects use their probes differently can be observed in their anatomical, such as the location of the transport channels. For example, in mosquitoes the extraction of food and injection of saliva is done with two different channels located in two individual and separate stylets. ${ }^{23}$ In contrast, both channels are in aphids formed by combining two of the probe stylets. ${ }^{24}$ Parasitic wasp ovipositors possess a single channel that is formed by all three functional elements, ${ }^{10}$ which can be used for both injecting liquids (venoms) ${ }^{25}$ and laying eggs. ${ }^{26}$ Additional to the transport function, the probes presumably also differ in the mode of oper- 
ation. Certain wasps, for example, use their ovipositors to physically restrict the host movements during egg-laying ${ }^{27}$ or to repair the probed silk cocoons using a felting-like technique. ${ }^{28}$ It is also likely that the insect differ in how they operate their probes within the substrates, although very little is known about this process. Here, we therefore focused on the biomechanics of probing with multi-element probes.

An existing hypothesis that is explained in detail below, proposes that the multielement nature of the probes makes them effective in solid (and stiff) substrates even at low probe diameters which make them highly flexible. Understanding the biomechanics of multi-element probe insertion in the substrate will elucidate the functional principles of the probes and may explain part of the observed structural variation of insect probes.

The shared anatomical features of multiple, interconnected probe elements indicates common general, mechanical requirements of probing that are elaborated on in chapter 2. This chapter also includes the theoretical framework of multi-element probe insertion and steering mechanism, together with examples and descriptions of insect probes in mosquitoes, hemipterans, and hymenopterans. In all species, the probing process can be divided into three distinct phases: puncturing the substrate, deeper insertion, and retraction of the probe. In this thesis, I will focus mostly on the second, explorative phase of probing.

It is unclear how insects explore the substrates with their long and thin (slender) probes. Probing with such probes is fascinating as slender structures can easily bend or break when subjected to axial loading (Euler buckling). Axial loading is necessary to push the probe in the substrate and it is unclear how insects avoid damage to their probes. Critical buckling load scales linearly with probe stiffness and its cross-sectional area and is inversely proportional to the square of the unsupported length of the probe and its end conditions (i.e. fixed ends allow for higher axial loads than pivoting or free ends). Insect probes are part of the exoskeleton and made out of cuticle-a highly versatile composite material whose stiffness can vary from $0.1 \mathrm{GPa}$ to $\sim 75 \mathrm{GPa} .{ }^{29}$ Despite being built from potentially very stiff materials, the critical buckling load of the ovipositors is low, because of their high slenderness ratios. Many insect therefore support their probes with additional structures, such as sheaths, gripping them with their legs. Additionally, insects evolved a 'smart' system that facilitates the insertion of their probes.

How a slender, multi-element probe avoids buckling has been hypothesized in the so called push-pull mechanism. ${ }^{30}$ According to this mechanism, the individual elements are moved reciprocally during probe insertion. The pulling (retraction) and pushing (protraction) of individual elements potentially reduces the external net 
pushing forces on the probe. These forces therefore stay below the threshold for buckling (breaking) of the probe, particularly the part outside the substrate. ${ }^{30}$ The part of the probe inside the substrate is supported and is less at risk of damage. The hypothesised push-pull insertion mechanism has, however, not been sufficiently quantified in living animals and it is currently still unknown to what extent the animals exploit the theoretical advantages of the push-pull mechanism.

Additional to buckling avoidance, many insects are also capable of steering their probes during insertion. ${ }^{31-33}$ This is beneficial because for at least three reasons. First, the exact location of the target within the substrate can generally not be determined from outside the substrate and the trajectory of probe insertion may need to be adjusted during target finding. This is particularly striking if the animals attack larval hosts that are moving freely within the substrate, such as parasitic wasps. ${ }^{34}$ Second, steering avoids complete reinsertion of the probe and a new puncture event, which poses the highest risk of buckling damage to the probe due to the large unsupported probe length and non-fixed distal end condition. And third, because natural substrates generally consists of hierarchically arranged layers and fibres that results in overall anisotropic material properties, ${ }^{35}$ i.e. are complex and might contain stiffer and less stiff parts, it might be easier and energetically less costly to avoid rather than pierce through stiff parts of the substrates.

\section{Steering}

Steering of the probes during substrate explorations has been observed in all above mentioned insect groups either in vivo ${ }^{36}$ or based on the insertion trajectories in the substrate. ${ }^{31,33,37}$ Anatomical studies of dead samples ${ }^{33,38,39}$ resulted in hypotheses of three major steering mechanisms that potentially explain the observed curving of the probes-all are discussed in detail in chapter 2. Briefly, all mechanism rely on the relative movements of the elements to induce curving of the probe. If elements are connected at their tips such that this limits their sliding, then pushing and pulling on opposing elements presumably induces tension and compression forces in these elements. This potentially results in curving of the probe as observed in mosquitoes and certain parasitic wasps. ${ }^{37,39}$ Alternatively, the probe can near its tip have stiff (more sclerotized) and soft (less sclerotized) regions near its tip, which differ in geometry (are curved or straight). ${ }^{38}$ When such differentially sclerotized elements are offset, the stiff and soft regions of neighbouring elements align and result in bending of the probe. The probe can also consist of pre-bent elements that form a straight probe when aligned. ${ }^{33}$ Because such elements are under stress in the aligned (resting) configuration, we term them pre-loaded. Protraction of such elements presumably leads to their curving. The described steering mechanisms 
are currently all hypothetical and it is not known whether they are present in the animals, nor how the animals might control them during probing.

Despite the evolutionary and ecological importance of probing and numerous working hypotheses of probe insertion, little is known about the probing capabilities in vivo. All suggested probing mechanisms are based on qualitative data and studies quantifying the probes and their usage are rare. ${ }^{\text {e.g. }} 40$ Furthermore, the structural and material properties of insect probes that are necessary to substantiate the above described hypothesized mechanisms, have rarely been measured.

\section{Ecological relevance and biomimetic potential}

Unravelling the biomechanics of probing with multi-element probes is important because it may provide a mechanical explanation for the success of insect probers and the observed variability of their probes. Understanding the probing mechanics can also elucidate the ecological interactions between the insects and their substrates, and in case of parasitic wasps also their hosts. Additionally, studying how slender probes avoid buckling may help in the development of similar man-made probes with similar functional demands. The potential for minimization (increasing probe slenderness) and controlled probing capabilities are of particular interest in biomedical engineering as evident from a growing number of steerable needle designs in the recent decades e.g. $41-44$ Slender and steerable tools inflict less damage and discomfort to the patient, and enables unprecedented control over surgical tools during medical procedures. Such tools could be become indispensable for targeting hard-to-get places deep within the body for delivering drugs or biopsies. Another possible medical application is the development of steerable electrodes that could be accurately placed deep within the patient's central nervous system.

\section{Research questions addressed in this thesis}

We set to answer how insects insert their slender, multi-element probes inside solid substrates using a number of methods explained below and as outlined in Fig. 2. We fine-tuned our research questions by first summarizing and putting into perspective the current knowledge on multi-element probes and their usage (chapter 2, Fig. 2). We pinpointed the functional and mechanical requirements of multi-element probes and elaborated on the previously hypothesized push-pull mechanism. By comparing the probing structures of insects such as mosquitoes, hemipterans, and parasitic wasps, we identified the common operating principles of multi-element probes that evolved in nature. These include interconnection of the elements and the use of tip asymmetry for steering. However, most of the literature on insect 


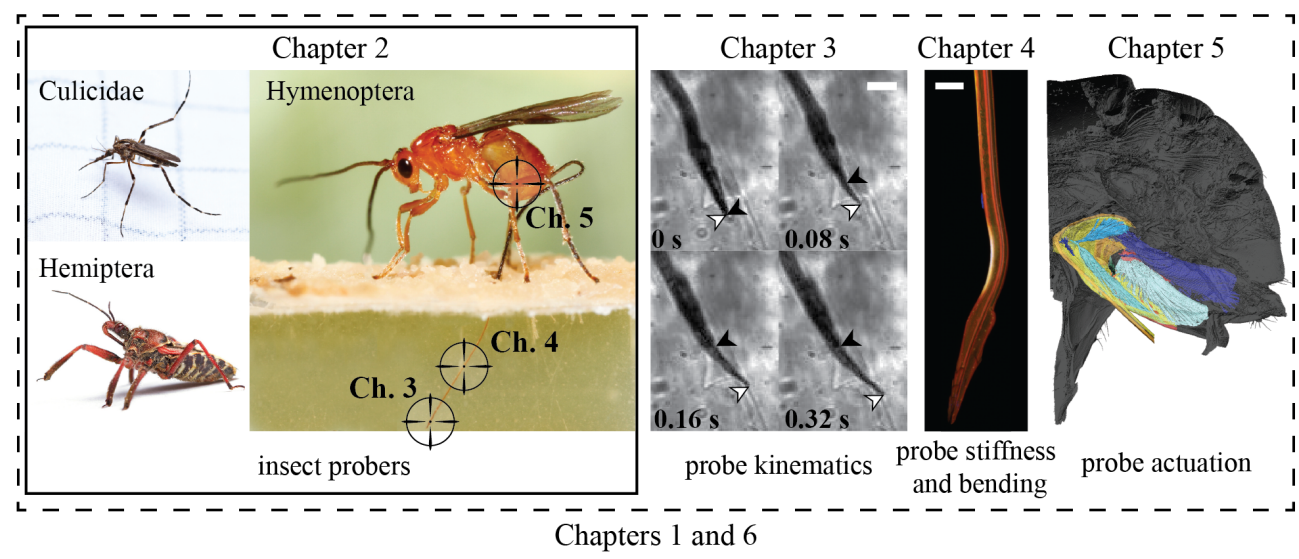

Fig. 2. Overview of the topic covered by thesis chapters. Chapters 1 and 6 are the introduction and discussion and cover all chapters. Chapter 2 is a comprehensive overview of the functional requirements of probing with multi-element with focus on three insect groups: mosquitoes (Culicidae), true bugs (Hemiptera), and parasitic wasps (Hymenoptera). It also includes the findings described in chapter 3, where we investigated the probing capabilities of the parasitic wasp Diachasmimorpha longicaudata in various substrates. Curved insertions were observed when the wasps probed by protracting their ventral valves, which caused shape changes in the ovipositor tip that presumably affected the probing direction. How such shape changes of the tip occur was studied in chapter 4 , where we measured structural and material properties of the ovipositor and its individual valves in D. longicaudata. In chapter 5, we investigated how the musculature and the exoskeletal elements at the base of the valves interact to generate the kinematics observed in chapter 3. Additionally, we quantified the muscle geometry and estimated the potential maximal forces that can be generated during probing to gain further insights into the push-pull mechanism of insertion. Photos of Culicidae and Hemiptera a courtesy of Alejandro Santillana (Insects Unlocked, The University of Texas, Austin, USA).

probes is descriptive or qualitative and quantitative measurements of probing kinematics, the structural and material properties of the probes and of the substrates are limited.

We chose to investigate the braconid parasitic wasp Diachasmimorpha longicaudata (Ashmead), formerly known as Biosteres (Opius) longicaudatus ${ }^{34}$ (Fig. 1A). This is an ideal candidate species because: (i) it possesses a very long $(5.7 \pm 0.6 \mathrm{~mm})^{34}$ and thin $(\sim 30 \mu \mathrm{m})$ ovipositor, which potentially easily buckles and is therefore suitable for studying anti-buckling mechanisms (Fig. 1A); (ii) the ovipositor consists of only three functional elements and is therefore a simpler system to study than the mouthparts of mosquitoes or hemipterans (Fig. 1B,C); (iii) the female wasps search and parasitize fruit-fly larvae hidden in fruits 45,46 and we expect the wasps to have some control over their probing direction (steering), (iv) the wasp probing behaviour can be induced in an experimentally controlled setting by supplying the wasps with translucent gels that enable the study of the probe kinematics within the substrate; and $(v)$ the wasps are easy to rear which is necessary for a constant supply of the experimental animals. Additionally, D. longicaudata wasps are often used as a biological pest control and knowing more about its probing capabilities may be also beneficial for agriculture. ${ }^{15,47}$ 
To test whether the wasps exploit the hypothesized push-pull mechanism during probing and investigate if and how the probing is affected by the substrate, we quantified their probing capabilities (chapter 3, Fig. 2). This is important because the push-pull mechanism is currently the only explanation for the buckling resistance of the probes, but it is unknown whether animals rely on it during probing. Additionally, knowing probing capabilities and how they are affected by the substrate has important implications on the understanding of the ecology of wasps and the development of steerable needles. For example, the wasp exploration range might decrease with substrate stiffness which potentially leads to a lower rate of parasitism and therefore lower fitness. We therefore compared the probing capabilities and steering kinematics of D. longicaudata in soft and a stiff substrates using high-speed videography.

D. longicaudata wasps control the shape of their ovipositor and the direction of probing by adjusting the protraction of specific probe elements. The ovipositor does not contain any musculature and curving occurs exclusively due to structural and material properties of the probe elements, their interactions between themselves and the substrate, or a combination of all these. A detailed analysis of the probe-substrate interactions, although very interesting and relevant for studying probing mechanisms, would require several additional years of study and could not have be done in the time-span of a single $\mathrm{PhD}$. Instead, I rather focused on investigating the structural and material properties of the probes. The structural properties of the ovipositor of $D$. longicaudata were studied using high-resolution synchrotron $\mu \mathrm{CT}$ scans and small-scale three-point bending experiments, while the material properties were qualitatively assessed with confocal laser scanning microscopy (CLSM). The findings presented in chapter 4 (Fig. 2) support a variation of the hypothesized steering mechanism relying on the differentially sclerotization of the elements. Additionally, our results indicate that the ovipositor in D. longicaudata evolved to enable steering during probing in solid substrates.

In chapter 5 (Fig. 2) we focus on how the important reciprocal movements of the valves are achieved. The musculature driving the ovipositor has been extensively described for several species and the operating principle has been hypothesized. However, the strength of the muscles driving the individual valves and their moment arms, both of which are important for testing the above mentioned push-pull mechanisms, have not been studied. We therefore investigated the musculature responsible for the movements of the ovipositor, focusing on both how the individual elements are controlled and what the is the order of magnitude of the forces acting on the elements during probing. The muscle dynamics and their maximal force output were estimated by analysing the configuration, shape, and size of ovipositor 
associated musculature in D. longicaudata. This was done by creating a 3D reconstruction of the relevant structures form high-resolution synchrotron $\mu \mathrm{CT}$ scans of animals in different probing configurations.

This thesis aims to understand the biomechanics of probing with multi-element structures to both answer biologically relevant questions and provide potential biomimetic solutions for development of man-made probes. In chapter 6 (general discussion, Fig. 2), I evaluate our findings, integrate them with the existing knowledge on multi-element probes, and discuss both their biological and engineering implications. I link our biomechanical findings to the ecology and evolution of parasitic wasps and discuss the findings in relation to other probing insects. I link the biological principles to the development of biomimetic probes, focusing on the multi-element and steerable needles for minimally invasive procedures. 


\section{References}

[1] Gordon RM \& Lumsden WHR (1939). A study of the behaviour of the mouth-parts of mosquitoes when taking up blood from living tissue together with some observations on the ingestion of microfiliarie. Annals of tropical medicine and parasitology 33, 259-278.

[2] Goodchild AJP (1966). Evolution of the alimentary canal in the hemiptera. Biological Reviews 41, $97-140$.

[3] Heatwole H, Davis DM, \& Wenner AM (1962). The behaviour of Megarhyssa, a genus of parasitic hymenopterans (Ichneumonidae: Ephialtinae). Zeitschrift fur Tierpsychologie - Journal of Comparative Ethology 19, 652-664.

[4] KrenN HW \& Aspöck H (2012). Form, function and evolution of the mouthparts of blood-feeding Arthropoda. Arthropod Structure \& Development 41, 101-118.

[5] Spradbery JP (1970). Host finding by Rhyssa persuasoria (L.) in ichneumonid parasite of siricid woodwasps. Animal Behaviour 18, 103-114.

[6] Rodrigues D, Sampaio DS, Isaias RMS, \& Moreira GRP (2008). Xylem and seed feeding by two passion vine leaffooted bugs, Holymenia clavigera and Anisoscelis foliacea marginella (Hemiptera: Coreidae: Anisoscelini), with notes on mouthpart morphology. Annals of the Entomological Society of America 100, 907-913.

[7] Polidori C, García AJ, \& Nieves-Aldrey JL (2013). Breaking up the wall: metal-enrichment in ovipositors, but not in mandibles, co-varies with substrate hardness in gall-wasps and their associates. PloS one 8, e70529.

[8] Sharkey MJ (2007). Phylogeny and classification of Hymenoptera. Zootaxa 1668, e548.

[9] СоввеN RH (1978). Evolutionary trends in heteroptera: Part II. Mouthpart-structures and feeding strategies. Wageningen, The Netherlands: H. Veenman \& Zonen B. V.

[10] Quicke DLJ, Fitton MG, Tunstead JR, Ingram SN, \& Gaitens PV (1994). Ovipositor structure and relationships within the Hymenoptera, with special reference to the Ichneumonoidea. Journal of Natural History 28, 635-682.

[11] Hodkinson DI \& CAsson D (1991). A lesser predilection for bugs: Hemiptera (Insecta) diversity in tropical rain forests. Biological Journal of the Linnean Society 43, 101-109.

[12] Huber JT (2009). Biodiversity of Hymenoptera. In RG Foottit \& PH AdLER, eds., Insect biodiversity: sicence and society, 632, Oxford: Blackwell Publishing Ltd., 1st edn.

[13] TAKKen W \& Verhulst NO (2013). Host preferences of blood-feeding mosquitoes. Annual Review of Entomology 58, 433-453.

[14] Raven JA (1983). Phytophages of xylem and phloem: a comparison of animal and plant sap-feeders. Advances in Ecological Research 13, 135-234.

[15] Ovruski S, Aluja M, Sivinski J, \& Wharton R (2000). Hymenopteran parasitoids on fruit-infesting Tephritidae (Diptera) in Latin America and the Southern United States: Diversity, distribution, taxonomic status and their use in fruit fly biological control. Integrated Pest Management Reviews 5, 81-107.

[16] Ghara M, Kundanati L, \& Borges RM (2011). Nature's Swiss Army knives: ovipositor structure mirrors ecology in a multitrophic fig wasp community. PloS one 6, e23642.

[17] Le LANNIC J \& NÉNON JP (1999). Functional morphology of the ovipositor in Megarhyssa atrata (Hymenoptera, Ichneumonidae) and its penetration into wood. Zoomorphology 119, 73-79.

[18] Robinson GG (1939). The mouthparts and their function in the female mosquito, Anopheles maculipennis. Parasitology 31, 212.

[19] Garzo E, Bonani JP, Lopes JRS, \& Fereres A (2012). Morphological description of the mouthparts of the Asian citrus psyllid, Diaphorina citri Kuwayama (Hemiptera: Psyllidae). Arthropod Structure \& Development 41, 79-86. 
[20] Quicke D, Leralec A, \& Vilhelmsen L (1999). Ovipositor structure and function in the parasitic Hymenoptera with an exploration of new hypotheses. Atti dell'Accademia Nazionale Italiana di Entomologia, Rendiconti 47, 197-239.

[21] LABANDEIRA CC (1997). Insect mouthparts: ascertaining the paleobiology of insect feeding strategies. Annual Review of Ecology and Systematics 28, 153-193.

[22] Kundanati L \& Gundiah N (2014). Biomechanics of substrate boring by fig wasps. Journal of Experimental Biology 217, 1946-1954.

[23] Snodgrass RE (1959). Anatomical life of the mosquito. Smithsonian miscellaneous collections 139, $1-87$.

[24] Pollard DG (1973). Plant penetration by feeding aphids (Hemiptera, Aphidoidea): a review. Bulletin of Entomological Research 62, 631.

[25] Robertson PL (1968). A morphological and functional study of the venom apparatus in representatives of some major groups of Hymenoptera. Australian Journal of Zoology 16, 133.

[26] Sмith EL (1970). Evolutionary morphology of the external insect genitalia. 2. Hymenoptera. Annals of the Entomological Society of America 63, 1-27.

[27] VAN Lenteren JC, Isidoro N, \& Bin F (1998). Functional anatomy of the ovipositor clip in the parasitoid Leptopilina heterotoma (Thompson) (Hymenoptera: Eucolidae), a structure to grip escaping host larvae. International Journal of Insect Morphology and Embryology 27, 263-268.

[28] Fritzen NR \& SAAKSJARvi IE (2016). Spider silk feltingfunctional morphology of the ovipositor tip of Clistopyga sp . (Ichneumonidae) reveals a novel use of the hymenopteran ovipositor. Biology letters 12, 20160350.

[29] Vincent JFV \& Wegst UGK (2004). Design and mechanical properties of insect cuticle. Arthropod Structure \& Development 33, 187-199.

[30] VINCENT JFV \& KING MJ (1995). The mechanism of drilling by wood wasp ovipositors. Biomimetics 3, 187-201.

[31] Elias LG, Teixeira SP, Kjellberg F, \& Santinelo Pereira RA (2012). Diversification in the use of resources by Idarnes species: bypassing functional constraints in the fig-fig wasp interaction. Biological Journal of the Linnean Society 106, 114-122.

[32] Leopold RA, Freeman TP, Buckner JS, \& Nelson DR (2003). Mouthpart morphology and stylet penetration of host plants by the glassy-winged sharpshooter, Homalodisca coagulata, (Homoptera: Cicadellidae). Arthropod Structure \& Development 32, 189-199.

[33] Pollard DG (1969). Directional control of the stylets in phytophagous Hemiptera. Proceedings of the Royal Entomological Society of London. Series A, General Entomology 44, 173-185.

[34] Leyva JL, Browning HW, \& Gilstrap FE (1991). Effect of host fruit species, size, and color on parasitization of Anastrepha ludens (Diptera: Tephritidae) by Diachasmimorpha longicaudata (Hymenoptera: Braconidae). Environmental Entomology 20, 1469-1474.

[35] AткINS A \& MAI YW (1985). Elastic and plastic fracture: metals, polymers, ceramics, composites, biological materials. Chichester: Ellis Horwood, Ltd./John Wiley and Sons.

[36] Choumet V, Attout T, Chartier L, Khun H, Sautereau J, Robbe-Vincent A, Brey P, Huerre M, \& BAIN O (2012). Visualizing non infectious and infectious Anopheles gambiae blood feedings in naive and saliva-immunized mice. PloS one 7 , e50464.

[37] Quicke DLJ \& FitTon MG (1995). Ovipositor steering mechanisms in parasitic wasps of the families Gasteruptiidae and Aulacidae (Hymenoptera). Proceedings of The Royal Society: Biological Sciences 261, 98-103.

[38] Quicke DLJ (1991). Ovipositor mechanics of the braconine wasp genus Zaglyptogastra and the ichneumonid genus Pristomerus. Journal of Natural History 25, 971-977.

[39] Waldbauer GP (1962). The mouth parts of female Psorophora ciliata (Diptera, Culicidae) with a new interpretation of the functions of the labral muscles. Journal of Morphology 111, 201-215.

[40] Pollard DG (1970). The mechanism of stylet movement in Psylla mali Schmidberger (Homoptera: Psyllidae). Zoological Journal of the Linnean Society 49, 295-307. 
[41] Sears P \& Dupont P (2006). A steerable needle technology using curved concentric tubes. 2006 IEEE/RSJ International Conference on Intelligent Robots and Systems 2850-2856.

[42] Frasson L, Neubert J, Reina S, Oldfield M, Davies BL, \& Rodriguez Y Baena F (2010). Development and validation of a numerical model for cross-section optimization of a multi-part probe for soft tissue intervention. 32nd Annual International Conference of the IEEE EMBS 32, 3202-3205.

[43] Roesthuis RJ, Abayazid M, \& Misra S (2012). Mechanics-based model for predicting in-plane needle deflection with multiple bends. 2012 4th IEEE RAS \& EMBS International Conference on Biomedical Robotics and Biomechatronics (BioRob) 69-74.

[44] Webster RJ, Romano JM, \& Cowan NJ (2009). Mechanics of precurved-tube continuum robots. IEEE Transactions on Robotics 25, 67-78.

[45] LAWRENCE PO (1981). Host vibration - A cue to host location by the parasite Biosteres longicaudatus. Oecologia 48, 249-251.

[46] Segura DF, Nussenbaum AL, \& Viscarret MM (2016). Innate host habitat preference in the parasitoid Diachasmimorpha longicaudata: functional significance and modifications through learning. PloS One 11, 0152222.

[47] Duan J, Ahmad M, Joshi K, \& Messing R (1997). Evaluation of the impact of the fruit fly parasitoid Diachasmimorpha longicaudata (Hymenoptera: Braconidae) on a nontarget Tephritid, Eutreta xanthochaeta (Diptera: Tephritidae). Biological Control 64, 58-64. 

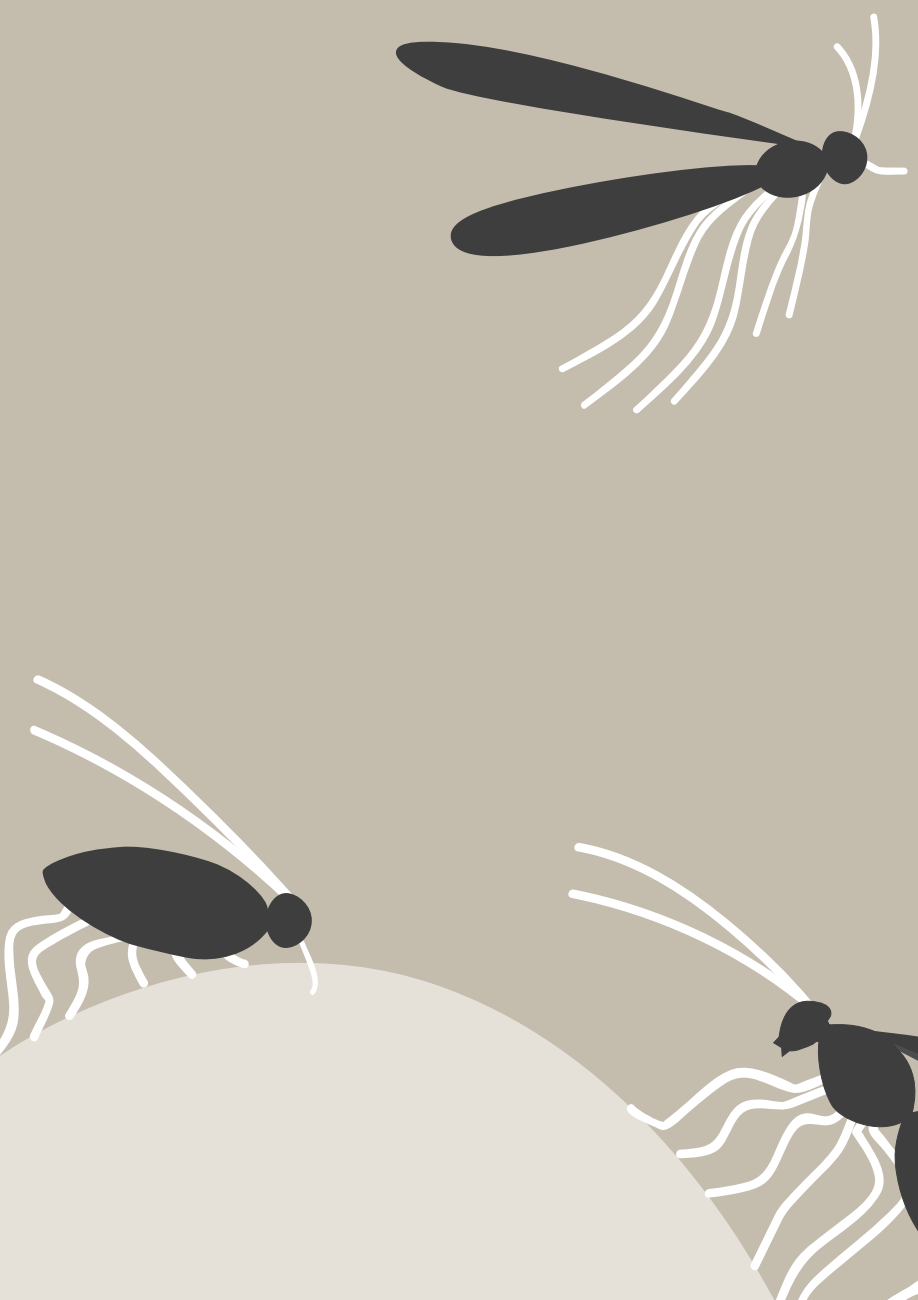


\section{chapter}

\section{Functional principles of steerable multi-element probes in insects}

Uroš Cerkvenik $^{1}$, Dimitra Dodou ${ }^{2}$, Johan L. van Leeuwen ${ }^{1}$, and Sander W.S. Gussekloo ${ }^{1}$

${ }^{1}$ Experimental Zoology Group, Department of Animal Sciences, Wageningen University, De Elst 1, 6708 WD Wageningen, The Netherlands

${ }^{2}$ Department of Biomechanical Engineering, Delft University of Technology, Mekelweg 2, 2628 CD, Delft, The Netherlands

Published as: Cerkvenik U, et al. (2018) Functional principles of steerable multielement probes in insects. Biological Reviews. doi:10.1111/brv.12467 


\section{Abstract}

Hemipterans, mosquitoes, and parasitic wasps probe in a variety of substrates to find hosts for their larvae or food sources. Probes capable of sensing and precise steering enable insects to navigate through solid substrates without visual information and to reach targets that are hidden deep inside the substrate. The probes belong to non-related taxa and originate from abdominal structures (wasps) or mouthparts (hemipterans and mosquitoes), but nevertheless share several morphological characteristics. Although the transport function clearly differs (egg laying and acquisition of liquid food), the functional demands on the mechanical behaviour of the probe within the substrate tend to be similar. The probe needs to be thin to limit substrate deformation, and long, in order to attain substantial path lengths or depths. We linked the morphology across taxa to the different functional requirements, to provide insights into the biology of probing insects and the evolution of their probes.

Current knowledge of insect probes is spread over many taxa, which offers the possibility to derive general characteristics of insect probing. Buckling during initial puncturing is limited by external support mechanisms. The probe itself consist of multiple (3-6) parts capable of sliding along one another. This multi-part construction presumably enables advancement and precise three-dimensional steering of the probe through the substrate with very low net external pushing forces, preventing buckling during substrate penetration. From a mechanical viewpoint, a minimum of three elements is required for 3D steering and volumetric exploration, as realised in the ovipositors of wasps. More elements, such as in six-element probes of mosquitoes, may enhance friction in soft substrates. Alternatively, additional elements can have functions other than 'drilling', such as saliva injection in mosquitoes. Despite the gross similarities, probes show differences in their cross sections, tip morphologies, relative lengths of their elements, and the shape of their interconnections. The hypothesis is that the probe morphology is influenced by the substrate properties, which are mostly unknown. Correlating the observed diversity to substrate-specific functional demands is therefore currently impossible.

We conclude that a multipart probe with sliding elements is highly effective for volumetric substrate probing. Shared functional demands have led to an evolutionary convergence of slender multi-element probes in disparate insect taxa. To fully understand $3 \mathrm{D}$ probing, it is necessary to study the sensory and material properties, as well as the detailed kinematics and dynamics of the various probes in relation to the nature of the selective pressure originating from the species-specific substrates. Such knowledge will deepen our understanding of probing mechanisms and may support the development of slender, bio-inspired probes. 


\section{Introduction}

Several insect taxa such as parasitic wasps (Hymenoptera), true bugs (Hemiptera), mosquitoes and flies (Diptera), and butterflies (Lepidoptera) include effective probers, which can find hosts for their larvae or food sources hidden in various substrates. The probes of these insects allow the delivery of eggs or fluids, such as venom, and/or the withdrawal of fluids, such as phloem sap and blood. Butterflies and flies that probe in solid substrates generally have rather short and stiff probes, with limited insertion depth. ${ }^{1,2}$ The presence of musculature inside the probe in butterflies ${ }^{1}$ presumably limits the length/width ratio $(1 / \mathrm{w})$, also called slenderness. This review focusses on slender probes that are generally present in the parasitic wasps, true bugs, and mosquitoes (Diptera: Culicidae). Parasitic wasps probe into a plethora of substrates, such as fruits, ${ }^{3}$ galls, ${ }^{4}$ and tree trunks, ${ }^{5}$ in search for host larvae. Hemipterans target various plant tissues, including phloem and xylem vessels, ${ }^{6}$ and mosquitoes generally search for blood vessels in the dermis and hypodermis of vertebrates. ${ }^{7}$ The highest ratios are found in species that need to probe deeply, such as the wood-probing wasp Megarhyssa atrata (Fabricius) $(1 / \mathrm{w} \approx 260),{ }^{8}$ and in xylem-feeding hemipterans, such as Stomaphis graffii (Cholodkovsky) $(1 / \mathrm{w} \approx 460) .^{9}$

The probing behaviour of insects raises several questions. How can these animals insert and advance their slender probes into solid and tough substrates with no or minimal damage to the probe? How do they accurately steer their probes towards a target without visual cues? Answers to these questions may offer insight into the life-history traits or life cycles of pests and parasitoids, and could help to improve drilling tools for medical and engineering applications.

Insect probes usually consist of multiple slender elements that are connected along their length but can slide along each other. The egg-laying structures (ovipositors) of parasitic wasps generally consist of three such elements, ${ }^{10}$ the mouthparts of hemipterans consist of four elements, ${ }^{11}$ and those of mosquitoes contain six elements. ${ }^{12}$ These probes are thought to have facilitated strong adaptive radiation of parasitic wasps and hemipterans, enabling the exploitation of a wide variety of niches. ${ }^{13,14}$ Hymenoptera is one of the most numerous insect orders, with about 145000 recognised species. ${ }^{15,16}$ For the order Hemiptera, about 82000 species have so far been described. ${ }^{17}$ The mosquito family Culicidae (Diptera), contains 'only' about 3200 recognised species. ${ }^{18}$ Although feeding on soft-bodied invertebrates occurs to some degree, ${ }^{19}$ mosquitoes primarily obtain their blood meals from vertebrate skin, a substrate that is similar across species. This may be a reason why diversification within mosquitoes is limited. 
We review the relationship between structure and function of multiple-element insect probes in terms of their mechanical and control properties. We discuss the capabilities of these probes in light of their functional demands for insertion and steering, and present an eco-morphological analysis based on basic probe characteristics. Similarities and differences across taxa in terms of functional morphology and mode of operation are critically evaluated.

\section{Function and theory of probing}

\section{Functional demands of probing insects}

Probing into solid substrates is a complex process where several control, transport, and mechanical challenges need to be overcome.

First, sensors and effective motor control are needed to search, reach, and assess a target accurately inside the substrate. ${ }^{20-22}$ Sensors are found, embedded in the cuticle, in almost all probes. ${ }^{12,23-25}$ Because of their relatively small size, we do not expect that sensors have a large impact on the probe morphology and probing mechanics. Muscles for probe motor control are large and thus typically situated outside the probe. Both sensors and muscles are of great importance for the life history of the animals, but as we focus on the insertion and steering of the probe itself we will not elaborate on sensors and muscles herein.

A second challenge of probing is the transport of fluid or eggs. ${ }^{26-28}$ Transport of (Newtonian) fluids through insect probes is often approximated using the HagenPoiseuille law ${ }^{29-32}$ :

$$
Q=\frac{\Delta P \pi R^{4}}{8 \mu L},
$$

where $Q$ is the volumetric flow rate through the probe, $\Delta P$ is the pressure difference over its length $L, R$ is the radius of the probe, and $\mu$ is the dynamic viscosity of the transported fluid. $Q$ is proportional to the fourth power of $R$, making $R$ the most sensitive parameter. A 10-fold increase in $R$ results in a 10000-fold increase in the flow rate if the $\Delta P$ is kept constant.

The transport of eggs through the canal depends on a different set of factors, including the difference in the diameter of the egg and canal, the elasticity of the egg and of the canal, the normal stress of the egg on the wall, and the friction between the two. We are unaware of any measurements of the material properties, deformations of eggs in parasitic wasps, presence of friction-reducing lubricants or of any estimations of the energy needed for passing an egg through the ovipositor. We hypothesise that for canals with a diameter smaller than the size of the unloaded egg, 
the work required for transport increases with decreasing canal diameter, although to our knowledge there are currently no data to confirm this.

Third, insect probers are faced with several additional mechanical challenges when it comes to drilling and steering, including (i) puncturing the substrate without damage to the probe, (ii) advancing the probe into the substrate, (iii) reaching the target, and (iv) extracting the probe from the medium. We briefly introduce these challenges below, and discuss the underlying physical principles and solutions on how to overcome these in 'Function and theory: Theoretical framework'. Other functional demands not directly related to probing and steering, such as the transport of fluids and eggs, will be addressed in 'Mode of operation: Additional considerations'.

Puncturing the substrate can be demanding because of substrate deformation and the presence of protective layers, such as stiff cell walls or viscoelastic structures in the substrate. Inserting a needle into a viscoelastic substrate (bovine liver) revealed that the force at the needle tip increases due to deformation of the substrate, until the substrate surface or internal tissue layers are ruptured, causing the force at the tip to decrease. ${ }^{33,34}$

During further insertion of the probe, the insect should overcome the friction along the length of the probe in addition to the cutting forces at the probe tip. Inserting a single-element probe into a solid substrate requires a pushing force that is large enough to overcome these forces and a sufficiently stiff probe to avoid buckling. 33,35 A probe consisting of multiple reciprocally moving elements may be inserted more easily into a substrate than a single-element probe, because the former can reduce the net friction and pushing forces on the entire probe, as explained in 'Function and theory: Theoretical framework: Advancing in the substrate' ${ }^{36,37}$

A host can be reached by steering the probe in the required direction. Steering requires a flexible probe. Flexibility depends on the stiffness of the probe materials and their geometry. Very little is currently known about the material properties of insect probes, except that they belong to the exoskeleton and consist of chitin and a variable protein matrix. The material stiffness of the cuticle, expressed as the Young's modulus, varies between about $0.1 \mathrm{GPa}$ in intersegmental membranes and $20 \mathrm{GPa}$ in the elytra of beetles. ${ }^{38}$ We found only a few studies that estimated the stiffness of insect probes; they reported a stiffness range of about 1-10 GPa, estimated based on either indentation force or material composition. ${ }^{24,36}$ With respect to geometry, a slender probe is more flexible than a wide probe of the same material and length, because of its smaller second moment of area as explained below (equation 2). 
The final requirement in probing is retraction of the probe from the substrate. Most insects simply pull their probes out of the substrate. ${ }^{24,39,40}$ This motion generates tensile forces within the probe and at its origin within the animal body. Slender probes can support much higher tensile than compressive stresses, and pulling on them is therefore not expected to induce damage to the probe. The probe-body connections are also expected to be capable of sustaining high tensile loads, considering that the probes are generally used multiple times throughout the lifetime of the animal. Because these tensile forces are expected not to damage the animal, we will not elaborate further on probe retraction.

The transport and insertion requirements, which have the greatest impact on the shape of insect probes, are contradictory. A large probe diameter may be advantageous for transport but makes the probe harder to insert and steer, due to reduced flexibility and increased friction. One disadvantage of inserting a probe with a large diameter is the reduced stress at the probe tip, which might hinder the puncturing and cutting of the substrate. This is less relevant for wasps, as all probes, as far as we know, have tapered tips ${ }^{36,41,42}$ that can concentrate the drilling forces onto a small area.

\section{Theoretical framework of the probing mechanics of multi-element probes}

\section{Puncturing the substrate}

Inserting a probe into a substrate requires an axial load, which, when not aligned along the probe axis, generates a bending moment. Even if the load is perfectly aligned with the probe axis, the probe still deforms (e.g. becomes thicker under compression). After structural buckling, the probe can return to its original shape when the load is removed, as long as the stress did not exceed the yield strength of the material. When the maximal load-bearing capabilities of the material (yield stress) are exceeded, plastic deformation occurs and the probe will not return to its original shape. The critical buckling load of an ideal (i.e. straight, homogeneous, and free from initial stress) beam as a function of its material and geometrical properties is given by the Euler equation (also see Fig. 1A):

$$
P_{c r}=\frac{\pi^{2} E I}{(K L)^{2}}
$$

where $P_{c r}$ is the critical load at which buckling occurs, $E$ is the elastic modulus of the material, $I$ is the second moment of area, which reflects the distribution of the crosssectional area around the bending axis (for details on calculating I see, for example, 

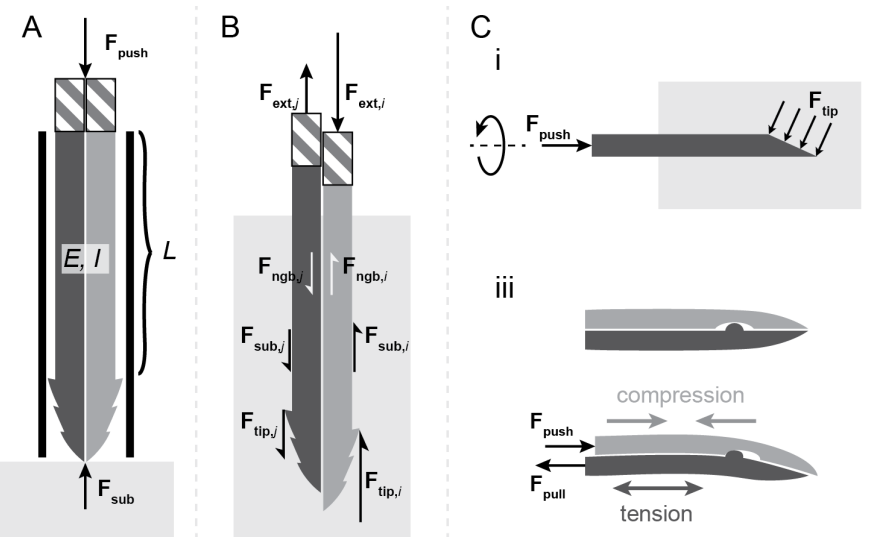

ii

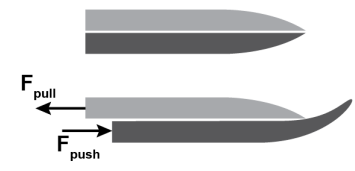

iii

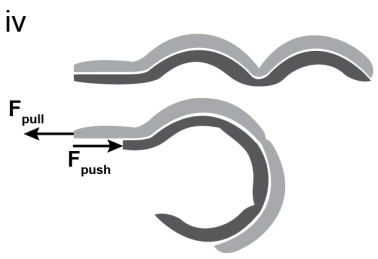

Fig. 1. General principles of puncturing, insertion, and bending of the probe in two dimensions. (A) Puncturing. The probe with Young's modulus E, second moment of area $I$, and length $L$ (real probes are longer than depicted here) is positioned at a suitable location on the substrate and pressed against it $\left(\mathbf{F}_{\text {push }}\right.$ and surface reaction force $\left.\mathbf{F}_{\text {sub }}\right)$. The probe outside the substrate is stabilised along its length (black vertical lines). (B) Advancement within the substrate. Further insertion is achieved using a 'push-pull' mechanism. ${ }^{36} \mathbf{F}_{\text {ext }}$, pushing or pulling force on the valve; $\mathbf{F}_{\text {sub }}$, friction force of the substrate along the probe shaft; $\mathbf{F}_{\mathbf{n g b}}$, inner inter-element friction force; $\mathbf{F}_{\mathbf{t i p}}$, force on the probe tip region. (C) Hypothesised bending mechanisms of insect probes. In all cases, the tip motion depends on the force generated between probe and substrate. The amount of bending (ii-iv) can be controlled by adjusting the amplitude of pro-/retraction of individual elements. Steering (ii-iv) can be achieved by the interplay of at least three elements (for clarity, only two are depicted). (i) An asymmetrical geometry of the probe tip region (bevel) leads to asymmetrical forces acting on it $\left(\mathbf{F}_{\text {tip }}\right)$ during insertion, causing the tip to bend from a straight path. Steering can be achieved via rotation of the probe about the longitudinal axis. ${ }^{43}$ (ii) 'Preloaded' elements ${ }^{44}$ curve towards each other when a low enough opposing force is present. This results in asymmetric forces as in (i). (iii) Longitudinally restricted movement of the elements by 'preapical stops'. ${ }^{45}$ The connections cause the build-up of tension and compression within the elements, thus generating bending moments within the ovipositor. (iv) Differential sclerotisation of elements causes the probe to bend when stiff (arches) and flexible regions (nodes) are aligned with each other. ${ }^{46}$

[47]), $L$ is the unsupported length of the beam, and $K$ is the effective length factor of the beam accounting for its end conditions ('pivoted' or 'fixed'). Beam diameter has a very high impact on the value of $P_{c r}$, as $I$ increases with approximately the fourth power of beam diameter.

\section{Advancing in the substrate}

The second challenge of probing insects is to advance their probe through a solid substrate. To do so, the insect should overcome the friction forces along the length of the probe and the cutting forces at its tip. Consider a cylindrical probe consisting of three or more slender elements that are interconnected longitudinally and can slide along each other Fig. 1B). Vincent and King suggested that advancement of such a multiple-element probe through a solid substrate is achieved by pushing forward and pulling backward the individual elements in an alternating fashion. ${ }^{36}$ The motion of a single element depends on the external force by the animal's body ( $\mathbf{F}_{\text {ext }}$; either pushing: $\mathbf{F}_{\text {ext }}>0$ or pulling: $\mathbf{F}_{\text {ext }}<0$ ), the friction forces along the 
length of the element by its neighbouring elements $\left(\mathbf{F}_{\mathbf{n g b}}\right)$ and by the substrate $\left(\mathbf{F}_{\text {sub }}\right)$, and the cutting force on the tip $\left(\mathbf{F}_{\text {tip }}\right)$. These forces can be quantified using Newton's second law:

$$
\mathbf{F}_{\text {ext }}+\mathbf{F}_{\text {ngb }}+\mathbf{F}_{\text {sub }}+\mathbf{F}_{\text {tip }}=m \mathbf{a},
$$

where $m$ is the mass of the element and a its acceleration. In a probe with $n$ elements the sum of all forces on the probe is:

$$
\sum_{i=1}^{n}\left(\mathbf{F}_{\mathbf{e x t}, i}+\mathbf{F}_{\mathbf{s u b}, i}+\mathbf{F}_{\mathbf{t i p}, i}\right)=\sum_{i=1}^{n} m_{i} \mathbf{a}_{i} .
$$

In equation $4^{*}$, the friction forces between neighbouring elements $\mathbf{F}_{\mathbf{n g b}, i}$ are not shown, because they cancel each other out, according to Newton's third law (action $=-$ reaction). Advancement of the probe into the substrate is even possible with a zero net external force or even a net pulling force $\left(\sum_{i=1}^{n} \mathbf{F}_{\text {ext }, i}<0\right)$ :

$$
\sum_{i=1}^{n} \mathbf{F}_{\mathbf{e x t}, i}=-\sum_{i=1}^{p}\left(\mathbf{F}_{\mathbf{s u b}, i}+\mathbf{F}_{\mathbf{t i p}, i}\right)-\sum_{j=1}^{r}\left(\mathbf{F}_{\mathbf{s u b}, j}+\mathbf{F}_{\mathbf{t i p}, j}\right)+\sum_{i=1}^{n} m_{i} \mathbf{a}_{i} \leq 0,
$$

with $p$ being the number of protracting elements and $r$ the number of retracting or stationary elements. In the case of a negligibly small contribution of the inertial term, this can be achieved by keeping the sum of the external friction and tip forces of the $p$ protracting elements below the reverse of the corresponding sum of the $r$ retracting and stationary elements, that is:

$$
-\sum_{i=1}^{p}\left(\mathbf{F}_{\mathbf{s u b}, i}+\mathbf{F}_{\mathbf{t i p}, i}\right) \leq \sum_{j=1}^{r}\left(\mathbf{F}_{\mathbf{s u b}, j}+\mathbf{F}_{\mathbf{t i p}, j}\right) .
$$

Equation 6 can be satisfied in various ways. First, the external surface area of the protracting element(s) can be smaller than that of the retracting and stationary element(s), which leads to lower friction on the protracting elements as compared to the friction on the retracting and stationary element(s). Second, by advancing only a minority of the elements (keeping $r$ high and $p$ low), the differences in the forces on the retracting and protracting elements are increased. Third, directional serrations on the elements 'anchor' them in the substrate during backwards pulling, whereas forward motion is possible with relatively low friction. ${ }^{36}$ Furthermore, $\mathbf{F}_{\text {tip }}$ may be reduced mechanically by using a sharp tip, or chemically by degrading the substrate. ${ }^{8}$

*In the published version, this equation was erroneously referenced as equation 3. 


\section{Steering the probe in multiple directions}

Steering a single-element probe in 3D space (Fig. 1Ci) ${ }^{48}$ is theoretically possible by combining forward translation, rotation along the longitudinal axis, and one-plane deflection (e.g. by means of a bevel that causes an off-axis reaction force on the tip). Rotation, however, requires a torsional moment on the probe shaft, which leads to a torsional stiffness-dependent angular lag between the tip and base of the probe or even structural failure of the probe. Probe rotation at the base can be avoided with alternative steering mechanisms available in multiple-element probes.

To simplify the discussion on possible steering mechanisms, consider a cylindrical probe consisting of two semi-cylindrical elements joined with little or no friction along the median plane of the probe (Fig. 1Cii-iv). One possible mechanism is based on the presence of a mechanical pre-stress in the distal region of the individual elements when the probe is straight (Fig. 1Cii) and is thus called the mechanism of preloaded elements. When such a distal region extends beyond the tip of the opposing element, the stress is reduced and the element bends inwards, forming a bevelled tip. ${ }^{44}$ The distribution of the pre-stress, the extent of the protraction, and the mechanical interaction with the substrate determine the bevel shape as well as the resulting trajectory of the probe.

Another possible steering mechanism uses 'preapical stops' to build up tension and compression by respectively pulling and pushing at the base of the two elements (Fig. 1Ciii). ${ }^{45}$ The generated tension difference at the opposing sides of the probe causes a bending moment distribution along the probe, which makes it curve. The resulting curvature of the probe depends on the exerted pulling and pushing forces, the second moment of area along the probe, and the interaction with the substrate.

Yet another mechanism relies on regional differences in bending stiffness and the 'stress-free' curvature of sclerotised regions along the two elements (Fig. 1Civ). ${ }^{46}$ The bending stiffness can be reduced by making the elements thinner (thereby decreasing the second moment of area) or by implementing a less-stiff material. Pulling and pushing forces on the two elements realign the mechanical regions of the elements, which results in a change in curvature.

All these proposed mechanisms are explained using two elements and, without rotation or twist along the longitudinal axis of the probe, can only result in bending in a single plane. When more elements are added, bending outside this single plane is possible. The multiple elements increase versatility in changing the shape of the probe tip (i.e. bevel angle) and consequently in adapting the probing direction. However, a larger number of elements also increases the complexity of the probe 
actuation, the required control mechanisms, and the transport through the probe, all of which will be addressed in 'Mode of operation: Additional considerations'. Probe element numbers vary in nature, which raises the question about the evolutionary pathways that led to this differentiation.

\section{Basic structure of multi-element probes}

In this section, we discuss the basic structure, the size, and the number of probe elements found in wasps, hemipterans, and mosquitoes. Differences in these characteristics may be affected by phylogeny, ontogeny, adaptation to substrate type, or other functional necessities, such as the transport of fluids or eggs.

\section{Parasitic wasps}

The ovipositor of parasitic wasps evolved from two pairs of tubular appendages at the ventral side of the animal's abdomen ${ }^{49}$ and can reach several body lengths. ${ }^{50,51}$ The appendages have many synonyms in the literature, but we here call them valves. ${ }^{10}$ The dorsal pair of valves is commonly fused, resulting in a three-element probe, although the extent of the fusion varies among species. ${ }^{10}$ In the oldest extant hymenopteran superfamilies such as Xyeloidea and Tenthredinoidea, ${ }^{14,52}$ the dorsal valves are partially fused, whereas wasps in the superfamily Chalcidoidea have only a membranous connection between them. ${ }^{10}$

In general, the fused dorsal valve contributes to about half of the ovipositor volume and half of its contact surface with the substrate. Each ventral valve contributes about a quarter to the ovipositor volume and likewise a quarter of the contact surface with the substrate, but exceptions occur. The egg canal is located between the three valves in the centre of the ovipositor ${ }^{10}$ (Fig. 2A-C).

Internally, the valves are filled with haemolymph, tracheas, and sensory nerves, without musculature. ${ }^{53,54}$ Each ventral valve is linked lengthwise to the dorsal one with a rail-like connection which allows the valves to slide along one another. $10,49,54,55$

The ovipositors are often enveloped and protected by a pair of flexible sheaths, appendages that also originate at the ventral side of the animal's abdomen. ${ }^{53,56}$ These sheaths are not inserted into the substrate. ${ }^{24,37,57}$ 

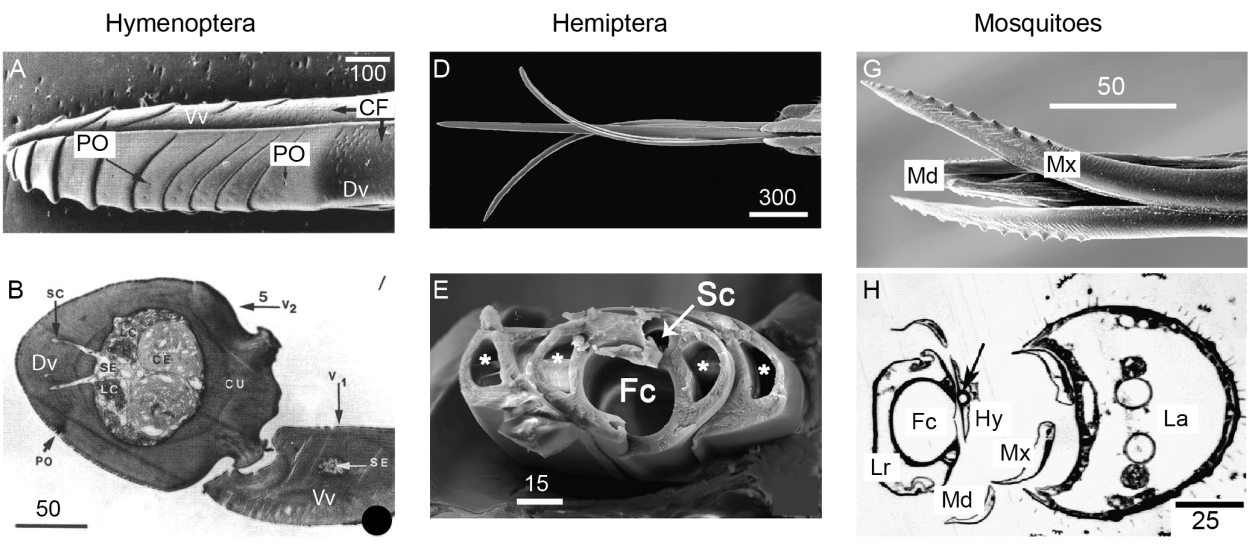

C

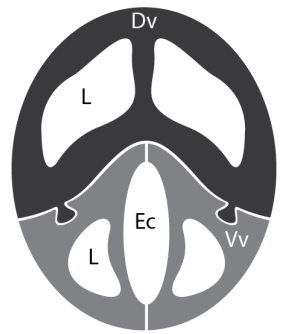

$\mathrm{F}$
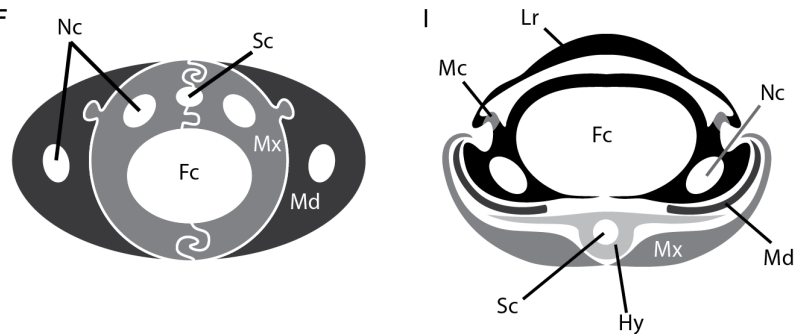

Fig. 2. Examples of insect probes. (A, B) Ovipositor of the parasitoid wasp Megarhyssa atrata (Ichneumonidae). ${ }^{5}$ (A) Scanning electron microscopy (SEM) image of the serrated dorsal (bottom, Dv) and ventral (top, $\mathrm{Vv}$ ) valves. PO, pores; CF, cuticular formations. (B) Cross section through the distal end of the ovipositor. One of the ventral valves is missing. CE, cuticular epithelium; CU, cuticle; LC, longitudinal intravascular canal; SC, intracuticle canal; SE, secretion; v1, ventral valve; v2, dorsal valve. (D, E) Mouthparts of Philagra albinotata (Uhler). ${ }^{58}$ (D) SEM image of the mouthparts outside the protective labium, with flared out mandibles and maxillae (middle) that are usually kept together. (E) Cross section through the stylets fascicle (location not given in the original article). The mandibles envelop the maxillae that form the food canal $(\mathrm{Fc})$ and the salivary canal $(\mathrm{Sc})$. Asterisks denote the dendritic canals. $(\mathrm{G}, \mathbf{H})$ Mosquito mouthparts (proboscis) of Anopheles stephensi (Liston). ${ }^{59}$ Maxillae (Mx) and mandibles (Md) enveloping the labrum (Lr; not indicated in $(\mathrm{G})$ and hypopharynx (Hy; not distinguishable in (G). La, labium; Fc, food canal. (C, F, and I) Generalised schematics of probe cross sections for Hymenoptera (C), Hemiptera (F) and mosquitoes (I). Ec, egg canal; L, lumen; Nc, neural canal; Mc, membranous connection. All scale bars in $\mu \mathrm{m}$.

\section{Hemipterans}

The piercing-and-sucking mouthparts of hemipterans most likely evolved from a chewing mouthpart type that also allowed for simple piercing. ${ }^{11}$ Lengths of hemipteran mouthparts are rarely reported, but can reach up to at least one body length. ${ }^{9}$ The general sequence of insect mouthparts (from anterior to posterior) is: a labrum (upper 'lip'), which acts as a cover structure, a hypopharynx, paired mandibles located lateral to the hypopharynx, paired maxillae, and a labium (lower 'lip'). ${ }^{11}$

In hemipterans, these mouthparts have been transformed into the proboscis, a complex piercing apparatus in which the mandibles and maxillae are modified into 
four elongated, stiff cuticular appendages, termed stylets ${ }^{13,60}$ (Fig. 2D). The maxillae taper from the base to their tip ${ }^{23,61}$ and generally interconnect with a rail-like mechanism similar to that observed in ovipositors of parasitic wasps. The maxillae form both the food and the salivary canals ${ }^{23,58,62}$ (Fig. 2E, F) and are located in the probe centre between the mandibles. The size of the food canal varies, depending on the animals' size and their diet. Canals with the smallest diameters $(<5 \mu \mathrm{m}$, but even reaching down to $0.5 \mu \mathrm{m})$ are found in hemipterans feeding on phloem sap ${ }^{9,62-64}$. Xylem-feeding hemipterans have food canals with diameters between 10 and $50 \mu \mathrm{m} .{ }^{58,65,66}$ Predatory, haematophagous hemipterans are grouped at the higher end of this range. ${ }^{59,67,68}$ By contrast, the salivary canals, through which mainly anticoagulants, enzymes, immuno-regulatory, and anti-inflammatory substances are inserted, ${ }^{69}$ have a constant width of less than $10 \mu \mathrm{m}$, irrespective of the diet.

The labrum is reduced to a cover plate at the base of the stylets, whereas the labium forms a sheath that houses the stylets and provides them with external support. Neither the labrum nor the labium penetrate the substrate. ${ }^{39,70}$

\section{Mosquitoes}

As in hemipterans, the probe of a female mosquito is called a proboscis, but contains six stylets. Mosquitoes insert their hypopharynx, labrum, mandibles, and maxillae into the substrate ${ }^{12,71}$ (Fig. 2G). The stylet length depends on the diet and is largest in females of blood-feeding species. ${ }^{72}$ By contrast, stylets are reduced or are even absent in non-blood-feeding males and in species feeding exclusively on plant material ${ }^{72}$; neither are addressed here.

The most dorsal stylet in the proboscis is the labrum, which forms the food canal with a diameter between 11 and $50 \mu \mathrm{m} .{ }^{32,73}$ The labrum is shaped as a doublewalled tube with a small opening on the ventral side. The two walls of the labrum form an inner lumen, are connected along their edges with a membrane, and are fused at their tips. The literature is not consistent on whether the hypopharynx ${ }^{41,74}$

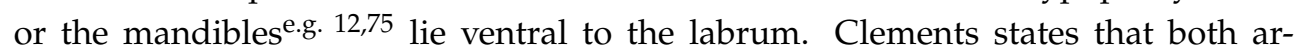
rangements are correct, with the hypopharynx lying ventral to the labrum at the base of the proboscis, and the mandibles at its tip. ${ }^{71}$ The maxillae are the most ventral of the stylets (Fig. 2G, H). The central lumen of the hypopharynx is the salivary canal, whereas the mandibles and the maxillae are solid slender rods. ${ }^{76}$ Not all stylets are interconnected; only shallow connections between the maxillae and the labrum have been reported. ${ }^{71}$

The labium terminates in two muscular labellae and houses the proboscis. ${ }^{12,71,75}$ 
Similar to the labium of hemipterans and sheaths of the wasps, the labium is not inserted into the substrate during probing. ${ }^{12}$

\section{Mode of operation}

A probing session can be divided into a number of steps, each with specific functional demands (see 'Function an theory of probing'). Execution of each step may vary among species. Similar considerations about the origin (phylogeny, ontology) and functional aspects (substrate adaptation, behaviour) as described in 'Basic structure of multi-element probes' apply here as well.

\section{Puncturing the substrate}

As previously mentioned, puncturing a substrate with a slender probe poses a risk of buckling and damaging the probe. Across taxa, the most common way of preventing buckling during puncturing is by enveloping the probe in a structure, to provide lateral support and increase the effective diameter of the probe (thus increasing its second moment of area).

\section{Wasps}

Sheaths protect the ovipositor in several wasp orders. ${ }^{56}$ In most cases, sheaths support the ovipositor during puncturing and at the beginning of its insertion into the substrate. The sheaths are not inserted into the substrate, but gradually fold over their full length in an arc-like shape away from the ovipositor base with increased insertion. ${ }^{37,77}$ Other buckling-prevention mechanisms include hardening the ovipositor tip (increasing $E$ in equation 2) with metal atoms, such as manganese and zinc, ${ }^{78}$ and shortening the probe's free length ( $L$ in equation 2 ) by locking the ovipositor at the base (coxa), ${ }^{79}$ midway between their legs, ${ }^{24,51}$ or in specialised grooves on their abdomen. ${ }^{5}$ If excessive bucking nevertheless does occur, the animals retract and straighten their ovipositor before resuming probing. ${ }^{24}$

\section{Hemipterans}

The segmented labium shaft that envelops the stylets is soft and moves away from the stylets by telescopic retraction into itself, the head, and sometimes the body. ${ }^{44,59,80}$ The labium tip is stiff and provides external support throughout the insertion process (decreasing $K$ in equation 2). ${ }^{9,81}$ Additional support can be provided by a flange of solidified saliva. ${ }^{23,28,82-84}$ The solidified saliva presumably stabilises the 
proboscis during probing, ${ }^{85}$ probably by fixing the end of the labium to the substrate (further decreasing $K$ in equation 2 ).

\section{Mosquitoes}

Similar to hemipterans, the mosquito labium supports the proboscis during puncturing and further insertion. The labium folds away from the proboscis in a hairpinlike curve during probing. ${ }^{86}$ In contrast to hemipterans, the distal end of the mosquito labium is soft and longitudinally divided into two muscular labellae. ${ }^{75}$ The labella presumably facilitate the puncturing of the skin by holding the stylets closely together. ${ }^{75}$ Alternatively, the labellae might assist in puncturing by moving laterally upon being pressed onto the skin, thus stretching the skin and easing crack formation by the maxillae. ${ }^{87}$ In both instances, the stylets are more or less fixed, which decreases $K$ in equation 2 .

\section{Advancing the probe into the substrate}

Alternate movements of elements during probing have been reported for wasps, 36,37 hemipterans, ${ }^{39,88}$ and mosquitoes. ${ }^{12,40}$ However, the varying number of elements among taxa indicates that animals use different modes of operation.

\section{Wasps}

In wasps, the valves can presumably be moved independently, as each valve has its own abdominal musculature. ${ }^{54,89-91}$ Inter-valve friction is likely to be kept low, possibly with lubricants. ${ }^{92-94}$

The hypothesised reciprocal movement in wasps has been quantified solely in $\mathrm{Di}$ achasmimorpha longicaudata Ashmead (Braconidae). ${ }^{37}$ Reciprocal movements were always observed when probing in relatively stiff substrates, but only occasionally in soft substrates. ${ }^{37}$ Wasps operate their valves at low speeds and accelerations ${ }^{37}$ and satisfy equation 6 by pushing one ventral valve forward at a time, while the dorsal valve and the other ventral valve remain stationary. Furthermore, the 'stationary elements' were observed to be effectively pulled back rather than remaining still. This may, in combination with tip serration, increase friction with the substrate (equation 6, Fig. 1B). The net pushing forces with which the valves are inserted by D. longicaudata were estimated to be very small $(<200 \mathrm{pN}) .{ }^{37}$ Advancing a single valve only a short distance beyond the tip of the stationary ones assures that the slenderness ratio of the protracted part is low, thereby preventing buckling. The maximal recorded amplitude of valve protraction in D. longicaudata is approximately $200 \mu \mathrm{m},{ }^{37}$ which is roughly $3.5 \%$ of the total ovipositor length. ${ }^{95}$ 


\section{Hemipterans}

In the four-element probe of hemipterans, the stylets are controlled independently by muscles located in the head. ${ }^{67,80,88}$ The stylets are bathed in salivary secretions which presumably have a lubricating function. ${ }^{82,96}$

For a four-element probe, various patterns of stylet advancement in the substrate have been observed. In air or during insertion in gels, hemipterans either move all the stylets reciprocally or use both maxillae together as one. ${ }^{80,88,97}$ We are aware of only one study that quantified the stylet motions and showed that in Psylla mali (Schmidberger) the mandibles are operated at half the frequency and twice the amplitude of the maxillae (i.e. $1.5 \mathrm{~Hz}$ and $5 \mu \mathrm{m}$ compared to $3 \mathrm{~Hz}$ and $2.5 \mu \mathrm{m}$, respectively. ${ }^{97}$ Hemipterans can probe by leading either with the mandibles or with the maxillae. ${ }^{39,70,88,97}$ Carnivorous hemipterans often use their mandibles only to anchor to their prey and then thrust their leading maxillae deep into the tissue. ${ }^{59,70}$

Cutting forces at the probe tip during probing are reduced mechanically (e.g. using sharp stylet tips or disrupting fibrous material by sawing), chemically (e.g. dissolving/softening the material), or with a combination of both. The saliva of certain species contains enzymes (e.g. pectinases) that degrade the connection between cells and thus soften the substrate. ${ }^{98}$ Species relying on pectinases generally take intercellular paths, ${ }^{39,82,99}$ whereas those mainly relying on mechanical work take predominantly intracellular paths to reach their target tissues. ${ }^{82}$ Flaring of the mouthparts deep in the target tissue has also been reported, which might occur to enlarge the opening of the food canal. ${ }^{67}$

\section{Mosquitoes}

All stylets of the mosquito proboscis, with the exception of the hypopharynx, are controlled by individual muscles. ${ }^{74,75}$ As the stylets lack tight inter-element connections (Fig. 2G, H, and I), we expect low friction between them.

We are not aware of any quantifications of stylet movements in mosquitoes. Observations of probing showed that mosquitoes rapidly move their maxillae and mandibles in alternate fashion along the labrum, which can be seen from the outside as vibrations of the stylet palp.e.g. 100,101 The labrum is tightly connected to the head and cannot move on its own. Instead, it is pushed inside the substrate by the vibration of the head. ${ }^{102}$ We hypothesise that the labrum muscles are predominantly used to bend this structure.

The stylets can become detached from each other and follow their own path in the substrate during insertion without apparent damage, despite bending at acute angles relative to each other. ${ }^{40}$ Whether this mechanical behaviour is intentional or 
not is hard to say. The proboscis tip flares out to a small degree during suction, which may help to open the entrance to the food canal. ${ }^{40}$

To summarise, alternate movements of the probe elements are a widespread feature in insect probes. The pro- and retraction of individual elements presumably reduces the net insertion forces of the stylets and avoids buckling of the probe. Pushing forces can be reduced by sharp element tips or by softening the substrate with chemical secretions.

\section{Steering the probe in multiple directions}

Several general steering mechanisms were described in 'Function and theory: Theoretical framework: Steering the probe in multiple directions'. Below we discuss the different mechanisms in our taxa of interest.

\section{Wasps}

At least some wasp species can direct their ovipositors in any direction with respect to their body orientation during probing (Fig. 3A-C) $37,50,103$. Steering in the dorsoventral plane is hypothetically achieved by 'preapical stops', 45 differential sclerotisation of the cuticle, ${ }^{46}$ or preloaded valves. ${ }^{44}$ Steering in a lateral direction could be achieved by rotating the ovipositor, predominantly using valves on one side of the ovipositor, or by bending the dorsal valve laterally. The latter may be facilitated by the membranous fusion of the dorsal valve (the so-called notal membrane). ${ }^{10,49,104}$ We assume that the membrane allows for minute, independent movements of two halves of the dorsal valve. In chalcidoid wasps (superfamily Chalcidoidea), this membrane runs almost along the entire length of the dorsal valve $^{10}$ and we propose this to be the reason for the reported exceptional bending capabilities. 103

The distal region of the ovipositor is usually geometrically asymmetric, e.g. 4,105,106 shaped as a bevelled tip. The mechanics of bending of bevel-tip needles as the result of passive interactions with the substrate are well known (Fig. 1Ci), 107 and this might also occur in ovipositors. Moreover, the valve movements may allow the wasps actively to change the shape and thus the bevel angle of the ovipositor tip. In D. longicaudata, the ventral valves appear to be preloaded, and their protraction strongly enhances the asymmetry of the distal region of the ovipositor. This is not true for the dorsal valve, and only drilling by leading with the ventral valves resulted in high curvature. ${ }^{37}$ Theoretically, controlling the amplitude of valve movements also controls the size of the bending radius and curvature length. 
A

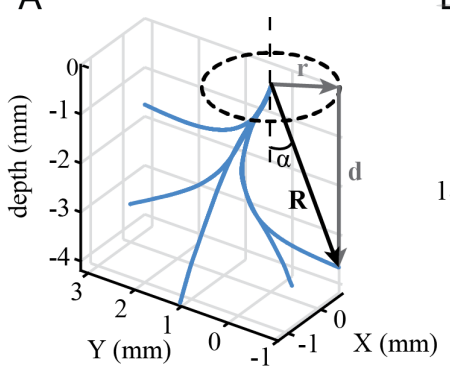

B

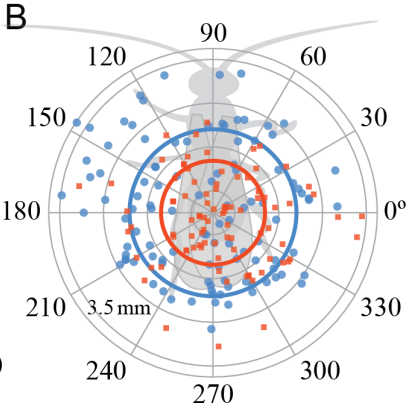

C

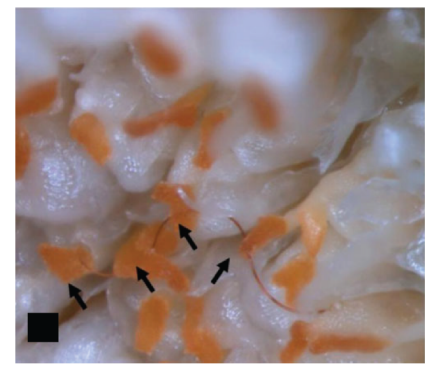

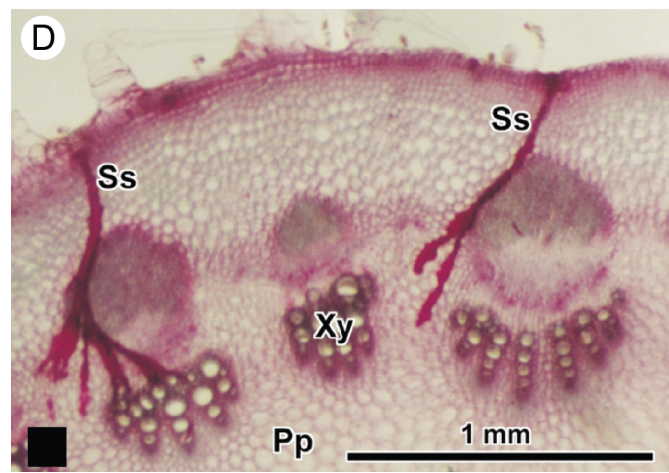

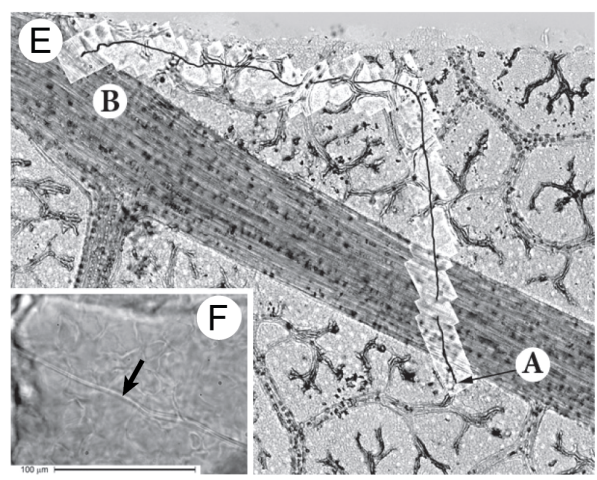

Fig. 3. Probing capabilities of hymenopterans and hemipterans. (A, B) Probing of parasitic wasp Diachasmimorpha longicaudata in artificial media. ${ }^{37}$ (A) A three-dimensional example of a probing session during which the ovipositor was partially retracted and reinserted in a different direction (blue lines). The endpoint of an individual insertion can be described by its horizontal distance to the start point (r), and the depth in the substrate (d). From these parameters the insertion angle $(\alpha)$ and the position vector of the insertion trajectory endpoint (R) can be calculated. (B) Top view of the insertion endpoints of many different wasps showing no directional preference and a range difference between the stiff (red) and soft (blue) substrates. (C) Parasitic wasp Idarnes flavicollis (Mayr) inserting its ovipositor into a fig fruit. ${ }^{103}$ The ovipositor takes a sinuous path between inflorescences indicating active steering during insertion. Scale not given in original publication. (D) Hemipteran Homalodisca coagulata (Say) stylet insertions into sunflower stem (light micrograph). ${ }^{23}$ The salivary sheaths (Ss) show a clear branched pattern when probing for xylem vessels (Xy). Pp, pith parenchyma. (E, F) Hemipteran Aulacaspis tubercularis (Newstead) probing a mango leaf. ${ }^{108}$ (E) Compilation of multiple high-resolution micrographs of stylet insertion (marked with a black line) through a mango leaf cleared with sodium hypochlorite Scale not given in original publication. A, insertion site; B, last field showing evidence of the stylets. (F) A micrograph showing part of the stylet bundle (arrow).

\section{Hemipterans}

Hemipterans are quite capable of fine control of the probing process, as observed from stylet paths within plant tissues that resemble those of ovipositor insertions (Fig. 3D-F). ${ }^{23,108}$ Due to the diversity in length and connections between stylets in hemipterans, various steering methods have been hypothesised. ${ }^{44}$

In Gerromorpha, an apical interlocking mechanism is created by interlocking lamellae. ${ }^{70} \mathrm{~A}$ preloading mechanism has been observed in the mandibles of Oncopeltus fasciatus (Dallas) ${ }^{88}$ and in the maxillae of Eupteryx melissae (Curtis), ${ }^{61,109}$ whereas 
a change of direction due to rotation of the inner maxillary stylets along their longitudinal axis with respect to the mandibles has been observed in Aphis gossypii (Glover) ${ }^{44}$ and hypothesised for E. melissae. ${ }^{109}$

Solidifying saliva secreted by phytophagous hemipterans ${ }^{23,39}$ might help in steering by providing a low-friction environment for stylet rotation or may act as a fulcrum as mentioned in 'Mode of operation: Additional considerations: Hemipterans'.

\section{Mosquitoes}

Flexibility is one of the most striking features of the mosquito probe: the proboscis can bend at least up to $90^{\circ}$ with respect to the original direction during probing. ${ }^{26,40}$ Steering appears to be mostly in the dorsoventral plane and is probably achieved by independent movement of the two lateral walls of the labrum. ${ }^{26,40,71}$ Each wall is reported to have its own musculature which can move them in opposite directions along their longitudinal axes. ${ }^{71,76}$ Because the labrum walls are fused at the distal end, their reciprocal movements induce bending due to generation of tensile and compressive forces within them. Contributions of other stylets of the proboscis cannot be assessed, due to the lack of quantification of mosquito probing kinematics. The maxillae and mandibles, albeit capable of back-and-forth movements, do not appear to contribute to steering. ${ }^{40}$ The hypopharynx does not have its own musculature, ${ }^{74}$ so we assume that it is moved by head movements and is not involved in steering.

\section{Summary}

A common solution for bending control in wasps, hemipterans, and mosquitoes is restricting the movements of the elements by interlocking or fusing them together. The presence of this steering mechanism is associated with probing in lowresistance substrates. It has been observed so far only in wasps parasitising hosts by exploiting existing tunnels in wood or nest entrances, ${ }^{45}$ in carnivorous hemipteran species feeding on soft tissues, ${ }^{70}$ and in haematophagous mosquitoes. ${ }^{71}$ Steering can also be done by protracting individual probe elements, which probably creates or enhances the asymmetry of the probe's distal end, thereby promoting bending due to interactions with the substrate. ${ }^{37}$ The influence of the substrate on steering mechanisms and capabilities, however, remains largely unknown.

\section{Additional considerations: transport of fluids and eggs}

As mentioned above, an important function of insect probes is the transport of either solid matter such as eggs, or a combination of particles and liquids such as 
phloem and blood. Apart from imposing a lower limit on probe width, the need to transport different substances is likely to have influenced the probe's structure and material composition. For example, to be effective, a probe should be able to withstand the inner pressure of the transported substance and should not leak. Here, we examine how the transported substances affect the shape and operational capabilities of insect probes.

\section{Wasps}

Before laying an egg, some wasps paralyse or kill their host by injecting venom. ${ }^{110}$ However, there is no connection between the ventral valves, which could potentially cause leakage. The ventral valves of many wasp species are equipped with thin cuticular flaps, located medially along the valves' common side, and projecting inwards into the egg canal (Fig. 4C). ${ }^{10,111,112}$ It is hypothesised that these soft flaps are forced out and thus overlap one another during injection of venom and egg laying. This effectively seals the crack between the ventral valves of the ovipositor along their entire length, creating a closed tube for fluid injection. ${ }^{10}$

The second stage, egg laying, also poses a challenge, because the diameter of the (unloaded) egg of parasitic wasps is usually much larger than the diameter of their egg canal. The egg must thus be squeezed into the egg canal, which results in a considerable shape change of the egg. ${ }^{27,113-116}$ Depending on the elasticity of the eggs, their passing along the egg canal could exert substantial mechanical stress on the inside walls of the ovipositor. The ovipositor expansion and its structural integrity also depends on the substrate surrounding the ovipositor. In relatively stiff media, the forces can be transferred to the substrate, which ensures that the ovipositor valves stay connected. In soft media, the ovipositor may expand, and the interlocking mechanisms must sustain the forces exerted by the egg. We could not find any empirical data on the material properties of the valve cuticle.

The passing of the egg through the egg canal is facilitated by small cuticular teeth/ combs lining the inside of the egg canal. ${ }^{111,115}$ As these teeth/combs point towards the ovipositor apex, the friction between the inner wall and the egg is higher upon protraction of the valve than upon retraction. Thus, the animals slowly transport the egg though the egg canal by alternate valve movements. ${ }^{115}$ Most of the work of egg laying is probably done by abdominal muscles that move the individual valves. 


\section{Hemipterans}

Hemipterans take up food and inject saliva through two separate canals formed by their maxillae. The salivary canal is smaller than the food canal and located within one of the maxillary interconnections. The muscular cibarial and salivary pumps power the suction of liquid food and the injection of saliva, respectively. ${ }^{60}$ Actual feeding dynamics of hemipterans are hard to measure, but it is proposed that pump pressures are correlated with the insect's food source, with higher pressures for more viscous fluids (up to 0.3 MPa for xylem feeders or haematophagous hemipterans. ${ }^{6,117,118}$

Phloem-feeding hemipterans do not seem to require active pumping of food, since the phloem itself is pressurised. This causes the sap to leak out on its own, although the net flow towards the animal can be aided by a pump. ${ }^{6}$ In these insects, the muscles of the cibarial pump may be used more as valves to reduce the flow of the phloem than to actuate pumping. ${ }^{39}$ However, some phloem feeders also exploit xylem, ${ }^{119}$ in which case pumping is expected. We hypothesise that the relatively small food canal diameters in phloem feeders may help reducing the plant-pressurised phloem flow to a manageable rate.

Xylem-feeding hemipterans need to actively suck their food from the host, because the pressure inside the xylem is presumably negative, or in other words, under tension. ${ }^{6}$ Thus, to obtain a sufficient amount of food, the animals need to generate a relatively large negative pressure. ${ }^{118}$ How the insects achieve this is not completely understood. Xylem-feeding hemipterans have powerful muscular pumps ${ }^{6,68}$ and relatively wide food canals that probably facilitate the uptake of liquids by lowering the required pressure differential during suction. ${ }^{65}$ In addition, they may feed primarily on sap that is under relatively low tension. ${ }^{118}$

In haematophagous hemipterans, ${ }^{117}$ the required negative pressure is expected to be higher than in phloem feeders, because the red blood cells in the blood make it a non-Newtonian fluid. ${ }^{120}$ Such fluids exhibit a higher viscosity with a decreasing flow velocity, ${ }^{121}$ requiring a relatively high pressure differential to start the flow. Wide canals facilitate the passage of blood cells through the probe and help to prevent clogging the food canal. Similar to xylem feeders, haematophagous hemipterans possess powerful muscular pumps ${ }^{6,68}$ and wide food canals ${ }^{59,68}$ that presumably facilitate the uptake of blood.

Saliva plays an important role in probing, but little is known about the injection dynamics of saliva in hemipterans. Phytophagous species are known to secrete two types of saliva, a gelling type and a watery type. The gelling saliva which forms the salivary sheath inside plant tissue may $(i)$ reduce the friction between the stylets 
and the surrounding tissue, ${ }^{122}$ (ii) act as a fulcrum for the manipulation of the mouthparts, ${ }^{67}$ and (iii) protect against leakage of pierced cells. ${ }^{39}$ The watery saliva contains enzymes which can (i) soften the cell walls, e.g. 99,123 (ii) help with digesting the food, ${ }^{85,122}$ and (iii) inhibit the immune response of the plant. ${ }^{82,122}$

\section{Mosquitoes}

Similar to hemipterans, the food canal of the mosquito is connected to the cibarial and pharyngeal pumps. ${ }^{74,124}$ Observations with micro-particle image velocimetry and real-time synchrotron micro-computed tomography $(\mu \mathrm{CT})$ scans revealed that the two pumps act synergistically to take up large amounts of food rapidly. ${ }^{124,125}$ Saliva is injected through the hypopharynx by the salivary pump. ${ }^{75,76}$ The mechanics of the saliva secretion is poorly understood, although it was suggested that female mosquitoes secrete it throughout the probing process. ${ }^{40,100}$ The saliva may help to keep the stylets in close apposition, ${ }^{12}$ but it also serves as an anticoagulant and anti-inflammatory substance. ${ }^{69,126}$

\section{Variations on a theme: coping with specific environments}

There are important morphological intra- and inter-order variations in the multipleelement probes. Below, we explore and evaluate these variations in the light of functional demands imposed on the animal's life-history traits and lifestyles.

\section{Cross section of the insect probe}

Although probes taper distally and are enlarged at the base, their cross section changes relatively little along most of their length. ${ }^{10,74}$ The probes of the taxa we described are generally oval in cross section, with the largest observed variability occurring in wasp ovipositors (Fig. 4A-D). ${ }^{10}$ This may be because parasitic wasps probe a great variety of substrates which pose different functional demands on the probing apparatus. Phytophagous wasps drilling in plant tissues, such as sawflies, have laterally compressed ovipositors, which is considered to be the ancestral state. ${ }^{14,16}$ Similarly, the proboscis of many hemipterans and of mosquitoes is also compressed, albeit dorsoventrally e.g. 59,70 (Fig. 2E, H). By contrast, the ovipositors of wasp species (parasitoid or phytophagous) that penetrate hard substrates have oval or circular cross sections. ${ }^{10,16}$ The latter shape maximises the internal lumen through which the egg is passed for a given amount of substrate displacement. ${ }^{10,16}$ 


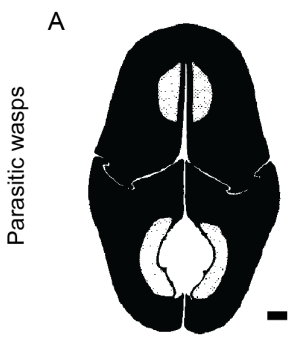

B

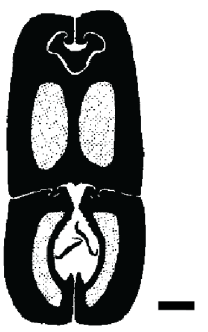

$\mathrm{C}$

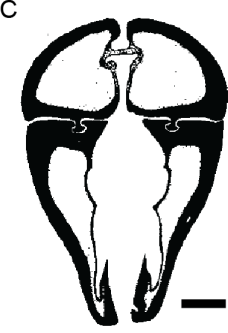

$\mathrm{D}$

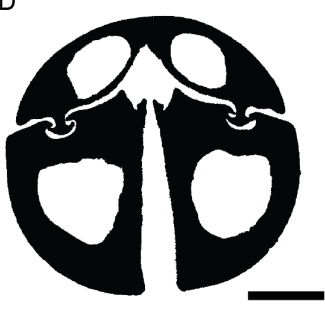

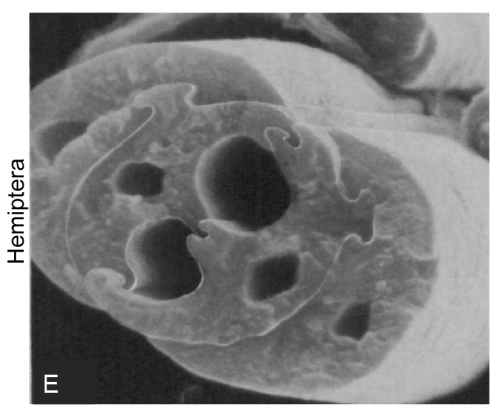
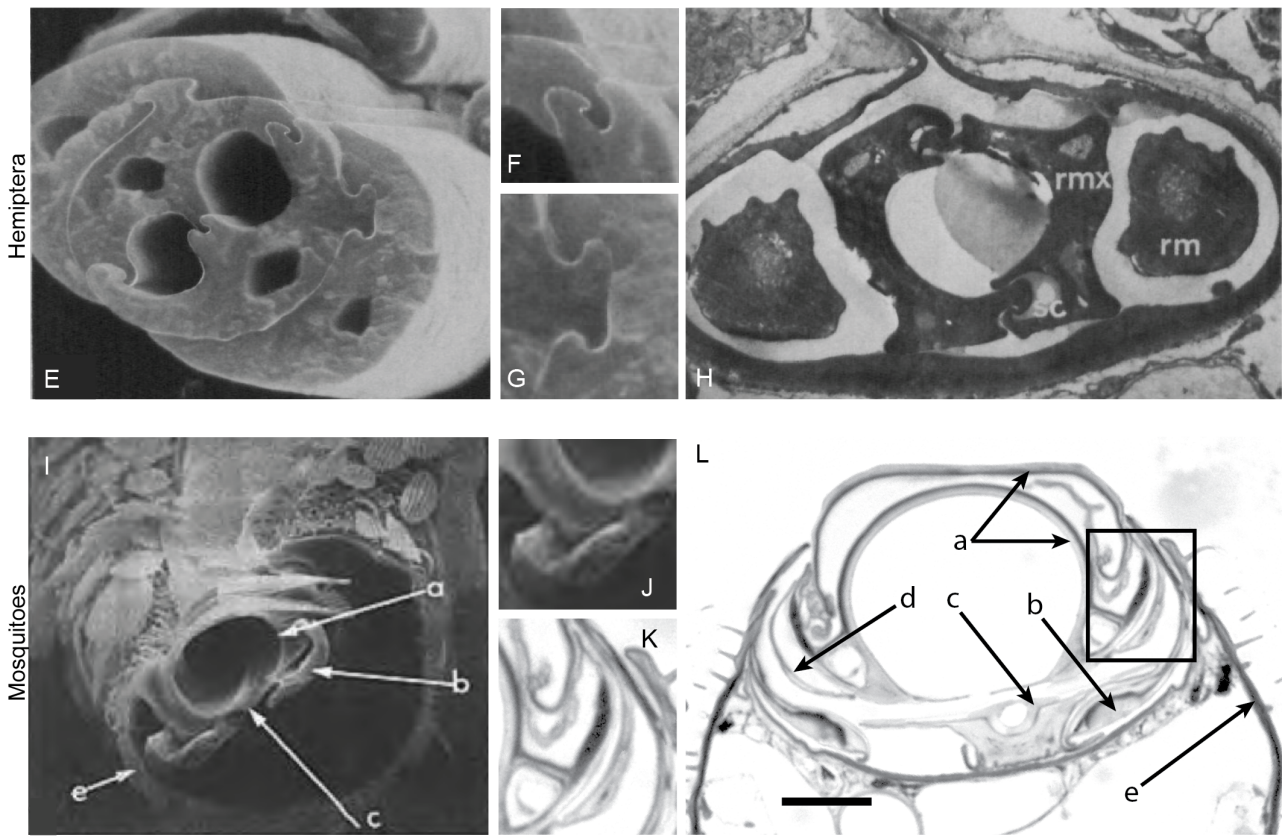

Fig. 4. Variation in element size and inter-element connections in probes of different taxa. (A-D) Cross sections through ovipositors of various parasitic wasps showing different kinds of inter-element connections. Note that a basic rail-like shape is present in all. The differences lie in the size of the connections, their orientation (diverging (A) or converging (D)), and the distance between them. A, B and C are from [10]: 142, 103, and 93, respectively. Scale bars A-D: $10 \mu \mathrm{m}$. (E-H) Inter-element connections between mouthparts of hemipterans. Note the complex shape of the connection between the maxillae (E, F). The 'rail type' can also be present, for example, between the mandibles and maxillae (G). In $\mathrm{H}$, the mandibles $(\mathrm{rm})$ are not connected to the maxillae ( $\mathrm{rmx}$ ) that also contain the salivary canal (sc); Figures from [70]: 138C (indicated magnification 1400×) and 143C (indicated magnification 4680 $\times$ ). (I-L) Cross-section of the mosquito proboscis. The stylets are weakly connected and are held together by the labium on the outside of the substrate, but can flare out when inside the tissue: $a$, labrum; $b$, maxilla; c, hypopharynx; d, mandible; e, labium. Details of the maxilla-labrum connection are shown in J (detail of I) and K (detail of L). Indicated magnification of I: 495×. Scale bar in L: $10 \mu \mathrm{m}$. I and J are from [41], Fig. 1.1. Species: (A) Coleocentrus sp. (Acaenitinae); (B) Lycorina sp. (Lycorininae); (C) Oedemopsis sp. (Tryphoninae); (D) Diachasmimorpha longicaudata (Braconidae) $(\mathrm{E}-\mathrm{G})$ species of Thaumastocoridae; $(\mathrm{H})$ Hebrus ruficeps Thomson (Hebridae), section at the base of the fourth labial segment; (I, J) Aedes atropalpus (Coquillet); (K, L) Culex pipiens (Linnaeus).

Individual elements differ in their cross sections within the probe, and their relative sizes and shapes show significant differences among parasitic wasps and hemipterans. ${ }^{10,70}$ Although the exact causation of this observed variation is un- 
known, it can be assumed that the morphology of individual elements is adapted to their specific function within the probe. The differences in cross sections of the ventral and dorsal ovipositor valves cannot easily be related to their functional roles, as all valves make extensive contact with the substrate during probing. Hemipterans and mosquitoes, however, have more-or-less nested probe elements, ${ }^{59,81}$ allowing us to make the following hypothesis: the elements that are considered to be the main stylets used in probing are largest, ${ }^{26,40,100}$ whereas the smaller elements normally have other functions, including clogging prevention ${ }^{127}$ and injection of saliva. ${ }^{12,74}$

In hemipterans, the cross-sectional shape of mandibular stylets seems to be associated with the evolutionary shift from a carnivorous to a phytophagous feeding style. Carnivorous (predatory and blood-sucking species) generally have oval or triangular ${ }^{68}$ mandibular cross sections, whereas phytophagous species have either comma-shaped ${ }^{81}$ or rectangular mandibles. ${ }^{9}$

We hypothesise that compressed probes have a preferred bending plane, whereas probes that are nearly circular are easier to steer in any direction and are thus suitable for animals aiming at moving targets, locations that cannot be reached in a straight line, or locations which are difficult to predict. Nevertheless, rectangular or even more complex cross sections are also present in wasps, ${ }^{10}$ probably in species that parasitise easily accessible hosts. This may indicate that the selection pressure for circular probes is most prominent in animals dealing with stiff substrates and those that need to steer in all directions.

\section{Alignment and interconnection of elements in insect probes}

For the multi-element probe to function as described above, its elements used for drilling need to be aligned and kept closely together (Fig. 4), although in mosquitoes some elements may flare out. ${ }^{40}$ The inter-element connections also need to be strong enough to withstand force from the substrate or internal forces that could separate them, yet loose enough to enable the relative movement of the elements along one other.

In wasps and hemipterans, interconnections are 'rail-like'. Wasps have a ridge with a mushroom-shaped cross section on the dorsal valve and a groove with an inverted mushroom shape in the ventral valves (Figs 2C, Fig. 4A-D). A similar, yet much more complex, 'tongue-and-groove' mechanism occurs between the elements of hemipteran mouthparts (Fig. 4E-H). The maxillae are usually interconnected by a twisted type of the 'tongue-and-groove' mechanism, where both 
maxillae contribute equally to the 'tongue' and the 'groove' part of the connection (Figs 2F, 4F). ${ }^{23,70,128,129}$ When present, the maxillae-mandible connection can be of the shallow rim-groove type or of the mushroom type similar to that in wasp ovipositors (Fig. 4G) ${ }^{70,128}$

We hypothesise that both the shape and the strength of the 'tongue-and-groove' connection depend on the stiffness and toughness of the substrate. A morphometric analysis of 113 ovipositor cross sections obtained from the literature indicated that the mushroom-shaped part is wider (thus possibly stronger) in wasps probing in materials classified as hard, such as wood, than in species probing in softer substrates or using exposed hosts. ${ }^{130}$ The same considerations probably also hold for the proboscis of hemipterans, although we have found no studies that quantify this.

Additionally, in wasps, the length of the connection seems to depend on the species' lifestyle. Endoparasitoid species, which lay eggs inside their hosts, possess ovipositors with 'tongue-and-groove' connections that extend all the way to the tip. This presumably facilitates the extrusion of eggs right at the tip of the ovipositor. In ectoparasitoids, which lay eggs on or adjacent to their hosts, the valve interconnections do not reach the ovipositor tip, allowing the eggs to be extruded earlier from the egg canal. ${ }^{105}$

Mosquitoes show little variance in the inter-stylet connections and possess shallow 'ridge-groove' connections between the labrum and the maxillae ${ }^{71}$ and between the labrum and the mandibles ${ }^{75}$ (Figs 2I, 4I, J). The labrum-maxillae connections are thought to hold the stylets in a bundle during probing, perhaps aided by a viscous fluid. ${ }^{12,41}$ The strength of inter-element connections is relatively weak and does not prevent the flaring out of the stylets. ${ }^{40}$ Because mosquitoes probe in skina relatively soft viscoelastic substrate-we hypothesise that there is no selective pressure to develop strong inter-element connections in their proboscis. The flaring of stylets in the mosquito proboscis might even enhance probing capabilities by exposing more of the stylet surface to the substrate, thus increasing anchorage of the probe necessary for the push-pull mechanism.

The close apposition between probe elements may cause strong internal friction forces and may create a risk of clogging of the probe if, for example, dust/dirt particles become wedged between the elements. In addition, the introduction of liquid between the probe elements might hinder their movements. ${ }^{131}$ How insects avoid such situations is not completely understood. The inner surfaces of ovipositors in many species bear small cuticular projections (ctenidia), which are thought to reduce friction between the valves by reducing their contact surface. ${ }^{111}$ Additionally, the inner surfaces of the ovipositors are also hypothesised to be hydrophobic, ${ }^{131}$ 
although this is unlikely in sucking insects, because hydrophobicity may induce cavitation under negative pressure. However, the use of liquids as means of keeping the stylets together or even facilitating their sliding (lubricants) has also been proposed. ${ }^{12,39}$ Unfortunately, none of the cited authors elaborates on the physical mechanisms in favour of their hypothesis.

\section{Elements: shape and length}

The greatest diversity in the morphology of the probes is found in their tips (Fig. 5). Attempts to correlate structural and material probe properties with substrate properties and life-history traits are only available for hymenopterans. ${ }^{90,91,105,132-134}$ For hemipterans and mosquitoes, we only found studies linking variation in tip shape and stylet lengths with feeding style..$^{70,72,135}$

Highly sclerotised ovipositors occur in certain wasp species that probe in tough substrates. ${ }^{4}$ Strengthening of the tip by deposition of heavy metal atoms was also reported. ${ }^{133}$ The latter not only enables piercing of hard substrates, but also reduces wear of the probe. ${ }^{78}$

The presence of serrations is also associated with substrate properties. An enlargement of the dorsal valve near its tip and large serrations on the ventral valves are generally found in wood-drilling ichneumon wasps, enabling stronger anchorage in the substrate during drilling. ${ }^{105}$ Valves with shallow or no serrations and without enlargements are mostly associated with species operating on soft and nearly homogeneous substrates such as immature, soft figs, whereas wasps probing in mature figs with heterogeneous tissues have not only more but also stronger and uneven serrations at the ovipositor tips. ${ }^{4,134}$ Although large protrusions on the ovipositor tip are generally associated with hard substrates, an enlargement of the dorsal valve near its tip is also present in D. longicaudata, which parasitises hosts hidden in soft fruits. ${ }^{37}$

In species that probe for feeding, the situation is more complicated, presumably because mouthparts are used for multiple functions. In predatory hemipterans, the serrated maxillae are not only used for drilling but also for lacerating the prey tissue. ${ }^{70}$ In blood-sucking representatives, the mandibles bear serrations, while serrations on the maxillae are reduced. ${ }^{68,70}$ In this case, the mandibles presumably help in steering, and maxillary serrations are not needed, because the animals feed on liquid food. The mandibles of plant-feeding species are strongly serrated, ${ }^{23,88,137}$ indicating that, similar to wasps, hard substrates require large serrations. We expect that the mandibles aid in penetration, whereas maxillae nested between them have a role in steering. It would be worth investigating whether the mouthparts 

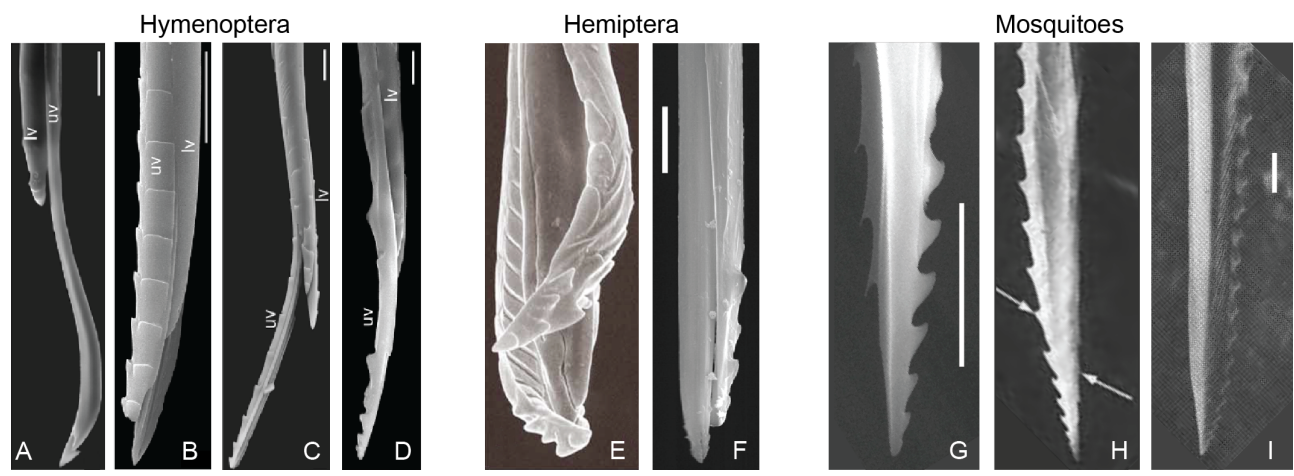

Fig. 5. Tip variations within insect orders. (A-D) Large variability is observed in the ovipositor tips of phytophagous and parasitoid wasps ranging from smooth valves, to small serrations, to strong serrations. ${ }^{4}$ The serrations can be present on either the upper valve (uv), the lower valves (lv), or all three valves. (A) Ceratosolen fusciceps (Mayr) (pollinating wasp), (B) Apocryptophagus fusca (Girault) (galler wasp), (C) Apocryptophagus agraensis (Joseph) (parasitoid), (D) Apocrypta westwoodi (Grandi) (parasitoid). (E, F) Different types of serrations on the outer wall of the mandibles of two species of hemipterans: (E) Oncopeltus fasciatus (Dallas) ${ }^{136}$ (contrast enhanced) and (F) Sogatella furcifera (Horváth). ${ }^{81}$ (G-I) Variation in shape of mosquito maxillae. Species differ in the number and size of stylet serrations. (G) Aedes albopictus (Skuse), ${ }^{101}$ (H) Aedes atropalpus (Coquillett), ${ }^{41}$ (I) Anopheles farauti (Laveran). ${ }^{135}$ Scale bars: $10 \mu \mathrm{m}$. Scale for $\mathrm{E}$ and $\mathrm{H}$ was not given in the original papers.

of phytophagous hemipterans show similar variation in their tip shape as observed in hymenopteran ovipositors. Mosquito species that feed on blood generally have more serrations on their maxillae than plant-feeding species that mostly feed on nectar or honeydew and do not penetrate plant tissues. ${ }^{41,138}$

In parasitic wasps, ovipositor length correlates with the required depth of probing. ${ }^{4}$ All valves are of equal lengths within individual ovipositors. Equal stylet lengths are also observed in bloodsucking mosquitoes and hemipterans, ${ }^{68,70,72}$ whereas mosquitoes feeding only on plants have stylets of different lengths. ${ }^{72}$ The degree of stylet length reduction is species specific and presumably correlates with the diet of the animal. ${ }^{72}$ Surprisingly, predatory hemipterans also have stylets of different lengths with mandibles shorter than maxillae. ${ }^{70}$ Mandibles are generally used for anchoring into their prey and maxillae for lacerating the tissue. ${ }^{70}$ The available data are too limited to draw any conclusions regarding possible correlations between the length of mouthparts and their behaviour.

\section{Discussion}

Probes of parasitic wasps, hemipterans, and mosquitoes share several structural features despite originating in non-closely related taxa. The shared functional demands of buckling avoidance and reaching targets deep in solid substrates presumably led to convergent evolution resulting in structural and kinematic similarities. 
All investigated groups use external support structures for buckling avoidance and alternative movements of individual probe elements during probe advancement through the substrate. Nevertheless, probes differ in their detailed morphology such as the shape of their cross sections, the number and length of elements forming the probes, the strength of inter-element connections, and tip geometry. Presumably, at least part of the observed morphological diversity can be explained by additional functionality of the probes and adaptations to specific substrates. In man-made needles it has been shown that larger serrations on the outer surface increase anchoring in the substrate, which is important for proper functioning of the push-pull mechanism. ${ }^{139}$ Studies linking these parameters to the substrate properties are lacking, and it is currently unclear what characteristics are substrateoptimised.

To understand insect probing mechanisms fully, we need to analyse the variation in structural and material properties of the probes and their detailed kinematics inside substrates. Although the general characteristics of probes and their functioning seem obvious, few data are currently available. Only a small number of studies describe and quantify probing. Many proposed mechanisms of insertion and steering (Fig. 1C) were derived from inspection of dead animals, 44,46,76,140,141 with properties of post-mortem material unlikely to be representative of the natural condition of insect probes and their steering mechanisms. Better insight into the actual mechanisms of insertion and steering could be achieved by quantitatively analysing the movement of probes inside the substrate, for example, by using highspeed videography and translucent media. ${ }^{37,97}$

Similarly, few studies focus on the structural and material properties of the probe. These can be obtained with high-resolution CT imaging or sequential transmission electron microscopy (TEM), using X-ray diffraction, ${ }^{142}$ small-scale three-point bending, ${ }^{143}$ or nano-indentation experiments. ${ }^{144}$ Due to the extremely small size of the probes, all these methods are either very expensive, difficult to execute, or both.

In addition, knowledge on forces involved in probing would complement our understanding of probe-substrate interactions. Quantification of forces in vivo is extremely difficult, but an estimation can be obtained from the size and arrangement of the muscles at the base of the probes. e.g. 109

Understanding the probing mechanics and the relationship between the probe shape and the substrate also has important engineering implications. It could, for example, prove helpful in the development of novel, bioinspired, minimally invasive tools for medical purposes. Slender, steerable needles requiring small insertion forces can have several benefits, including minimal tissue damage, bypassing important struc- 
tures such as blood vessels and nerves, and accessing hard-to-reach places within the body. ${ }^{145,146}$

Multi-element steerable needles are already in use and more are under development, ${ }^{147}$ but none are as slender and perform as well as insect probes. In most cases, steering is achieved by exploiting needle-tissue interactions arising from a bevelled tip (Fig. 1Ci; 'Function and theory: Theoretical framework: Advancing in the substrate' $).{ }^{34}$ In all cases, the needle is still pushed into the substrate with an external net pushing force. This requires stiff needles which hinders the minimisation of their diameters. Furthermore, the reported relative curvatures of needle insertions ${ }^{148,149}$ are generally much lower than found in wasps, ${ }^{37}$ although some prototypes perform better when bending in one plane. ${ }^{107}$ The restrictions in curving probably affect the accuracy and limit the versatility of these novel surgical tools. Needles based on insect probes may help to solve the challenges of minimisation, buckling, and steering. Development of multi-element needles capable of adjusting their tip asymmetry is already underway. ${ }^{146,147,150}$ Inserting such needles using reciprocal motion of elements decreases tissue strain and net insertion forces that might lead to tearing and damage. ${ }^{146,151}$ Insect-inspired probes might lead to the development of automated self-propelling probes, which will not only be useful for medical purposes, but may also be scaled up and employed anywhere where substrates need to be explored in depth, for example in construction or geology.

\section{Conclusions}

(1) Initial puncturing of the substrate is always facilitated by external support mechanisms that prevent buckling and breaking of the probe.

(2) A multi-element design enables the probe to pull itself into the substrate by pro- and retracting individual elements and to steer by changing the tip shape resulting from the offset of the elements.

(3) Assuming no rotation or twist around the longitudinal axis of the probe, a minimum of three elements is required for 3D steering. Some probes possess more than three elements, which might be specialised for other functions such as injection of saliva and may have little influence on the steering of the probe.

(4) The probe tip is presumably adapted to a specific type of substrate, but information on the material properties of the substrate (and of probes themselves) is lacking.

(5) Understanding the probing mechanisms across various taxa will offer insights into their evolution, lifestyle, and ecological interactions, and may also aid the development of human-made probes. 


\section{Acknowledgements}

We thank Henk Schipper and Frank van der Berg for their help with histology, and the members of the Experimental Zoology Group for their critical review of this manuscript. This work was supported by the NWO Domain Applied and Engineering Sciences (NWO TTW). 


\section{References}

[1] KRENN HW (2010). Feeding mechanisms of adult lepidoptera: structure, function, and evolution of the mouthparts. Annual Review of Entomology 55, 307-327.

[2] Karolyi F, Colville JF, Handschuh S, Metscher BD, \& Krenn HW (2014). One proboscis, two tasks: Adaptations to blood-feeding and nectar-extracting in long-proboscid horse flies (Tabanidae, Philoliche). Arthropod Structure \& Development 43, 403-413.

[3] Ovruski S, Aluja M, Sivinski J, \& Wharton R (2000). Hymenopteran parasitoids on fruitinfesting Tephritidae (Diptera) in Latin America and the Southern United States: Diversity, distribution, taxonomic status and their use in fruit fly biological control. Integrated Pest Management Reviews 5, 81-107.

[4] Ghara M, Kundanati L, \& Borges RM (2011). Nature's Swiss Army knives: ovipositor structure mirrors ecology in a multitrophic fig wasp community. PloS one 6, e23642.

[5] Heatwole H, Davis DM, \& Wenner AM (1962). The behaviour of Megarhyssa, a genus of parasitic hymenopterans (Ichneumonidae: Ephialtinae). Zeitschrift fur Tierpsychologie - Journal of Comparative Ethology 19, 652-664.

[6] RAVEN JA (1983). Phytophages of xylem and phloem: a comparison of animal and plant sapfeeders. Advances in Ecological Research 13, 135-234.

[7] TAKKEN W \& Verhulst NO (2013). Host preferences of blood-feeding mosquitoes. Annual Review of Entomology 58, 433-453.

[8] Le LANNIC J \& NÉNON JP (1999). Functional morphology of the ovipositor in Megarhyssa atrata (Hymenoptera, Ichneumonidae) and its penetration into wood. Zoomorphology 119, 73-79.

[9] Brożek J, Mróz E, Wylf̧żé D, Depa $€$, \& WȩGierek P (2015). The structure of extremely long mouthparts in the aphid genus Stomaphis Walker (Hemiptera: Sternorrhyncha: Aphididae). Zoomorphology 134, 431-445.

[10] Quicke DLJ, Fitton MG, Tunstead JR, Ingram SN, \& Gaitens PV (1994). Ovipositor structure and relationships within the Hymenoptera, with special reference to the Ichneumonoidea. Journal of Natural History 28, 635-682.

[11] LABANDEIRA CC (1997). Insect mouthparts: ascertaining the paleobiology of insect feeding strategies. Annual Review of Ecology and Systematics 28, 153-193.

[12] Robinson GG (1939). The mouthparts and their function in the female mosquito, Anopheles maculipennis. Parasitology 31, 212.

[13] Goodchild AJP (1966). Evolution of the alimentary canal in the hemiptera. Biological Reviews 41, $97-140$.

[14] Sharkey MJ (2007). Phylogeny and classification of Hymenoptera. Zootaxa 1668, e548.

[15] Huber JT (2009). Biodiversity of Hymenoptera. In RG Foottit \& PH AdLer, eds., Insect biodiversity: sicence and society, 632, Oxford: Blackwell Publishing Ltd., 1st edn.

[16] Vilhelmsen L \& TuRRIsi GF (2011). Per arborem ad astra: morphological adaptations to exploiting the woody habitat in the early evolution of Hymenoptera. Arthropod Structure \& Development 40, $2-20$.

[17] Brambila J \& Hodges SG (2008). Bugs (Hemiptera). In J Capinera, ed., Encyclopedia of Entomology, 591-611, Dordrecht: Springer Netherlands.

[18] Foster WA \& Walker ED (2002). Mosquitoes (Culicidae). In G Mullen \& D LanCE, eds., Medical and veterinary entomology, 597, Elsevier Science.

[19] George J, Blanford S, Thomas MB, \& Baker TC (2014). Malaria Mosquitoes Host-Locate and Feed upon Caterpillars. PLoS one 9, e108894.

[20] Leon DD (1935). The biology of Coeloides dendroctoni Cushman (Hymenoptera-Braconidae) an important parasite of the mountain pine beetle (Dendroctonus monticole Hopk.). Annals of the Entomological Society of America XXVIII, 411-424. 
[21] WANG X \& Keller MA (2002). A comparison of the host-searching efficiency of two larval parasitoids of Plutella xylostella. Ecological Entomology 27, 105-114.

[22] Ruschioni S, Van Loon JJA, SMid HM, \& Van Lenteren JC (2015). Insects can count: sensory basis of host discrimination in parasitoid wasps revealed. PLoS One 10, e0138045.

[23] Leopold RA, Freeman TP, BucKner JS, \& Nelson DR (2003). Mouthpart morphology and stylet penetration of host plants by the glassy-winged sharpshooter, Homalodisca coagulata, (Homoptera: Cicadellidae). Arthropod Structure \& Development 32, 189-199.

[24] Kundanati L \& Gundiah N (2014). Biomechanics of substrate boring by fig wasps. Journal of Experimental Biology 217, 1946-1954.

[25] Zhang L, Feng YQ, Ren Ll, Luo YQ, Wang F, \& Zong Sx (2014). Sensilla on antenna, ovipositor and leg of Eriborus applicitus (Hymenoptera: Ichneumonidae), a parasitoid wasp of Holcocerus insularis staudinger (Lepidoptera: Cossidae). Acta Zoologica 96, 253-263.

[26] Gordon RM \& LuMSDEN WHR (1939). A study of the behaviour of the mouth-parts of mosquitoes when taking up blood from living tissue together with some observations on the ingestion of microfiliarie. Annals of tropical medicine and parasitology 33, 259-278.

[27] BRonner R (1985). Anatomy of the ovipositor and oviposition behavior of the gall wasp Diplolepis rosae (Hymenoptera: Cynipidae). Can. Ent. 117, 849-858.

[28] TJallingII WF (2006). Salivary secretions by aphids interacting with proteins of phloem wound responses. Journal of Experimental Botany 57, 739-745.

[29] Daniel TL \& Kingsolver JG (1983). Feeding strategy and the mechanics of blood sucking in insects. Journal of Theoretical Biology 105, 661-677.

[30] Loudon C \& McCulloh K (1999). Application of the Hagen-Poiseuille equation to fluid feeding through short tubes. Annals of the Entomological Society of America 92, 153-158.

[31] KiкUсні K \& Mochizuкi O (2008). Micro-PIV measurements in micro-tubes and proboscis of mosquito. Journal of Fluid Science and Technology 3, 975-986.

[32] LeE SJ, KIM BH, \& LeE JY (2009). Experimental study on the fluid mechanics of blood sucking in the proboscis of a female mosquito. Journal of biomechanics 42, 857-864.

[33] Okamura AM, Simone C, \& O'Leary MD (2004). Force modeling for needle insertion into soft tissue. IEEE Transactions on Biomedical Engineering 51, 1707-1716.

[34] Elgezua I, Ковayashi Y, \& Fujie MG (2013). Survey on current state-of-the-art in needle insertion robots: open challenges for application in real surgery. Procedia CIRP 5, 94-99.

[35] Casanova F, Carney PR, \& Sarntinoranont M (2014). In vivo evaluation of needle force and friction stress during insertion at varying insertion speed into the brain. Journal of Neuroscience Methods 237, 79-89.

[36] VINCENT JFV \& KING MJ (1995). The mechanism of drilling by wood wasp ovipositors. Biomimetics 3, 187-201.

[37] Cerkvenik U, Van de Straat B, Gussekloo SWS, \& van Leeuwen JL (2017). The mechanisms of ovipositor insertion and steering of a parasitic wasp. Proceedings of the National Academy of Sciences 114 (37), E7822-E7831.

[38] Vincent JFV \& Wegst UGK (2004). Design and mechanical properties of insect cuticle. Arthropod Structure \& Development 33, 187-199.

[39] Pollard DG (1973). Plant penetration by feeding aphids (Hemiptera, Aphidoidea): a review. Bulletin of Entomological Research 62, 631.

[40] Choumet V, Attout T, Chartier L, Khun H, Sautereau J, Robbe-Vincent A, Brey P, Huerre M, \& BaIN O (2012). Visualizing non infectious and infectious Anopheles gambiae blood feedings in naive and saliva-immunized mice. PloS one 7 , e50464.

[41] Hudson A (1970). Notes on the piercing mouthparts of three species of mosquitoes (Diptera: Culicidae) viewed with te scanning electron microscope. The Canadian Entomologist 102, 501-9. 
[42] Ahmad A, Kaushik S, Ramamurthy VV, Lakhanpaul S, Ramani R, Sharma KK, \& VidYARTHI AS (2012). Mouthparts and stylet penetration of the lac insect Kerria lacca (Kerr) (Hemiptera:Tachardiidae). Arthropod Structure \& Development 41, 435-441.

[43] Webster RJ, Kim JS, Cowan NJ, Chirikjian GS, \& OKamura AM (2006). Nonholonomic modeling of needle steering. The International Journal of Robotics Research 25, 509-525.

[44] Pollard DG (1969). Directional control of the stylets in phytophagous Hemiptera. Proceedings of the Royal Entomological Society of London. Series A, General Entomology 44, 173-185.

[45] Quicke DLJ, Fitton M, \& Harris J (1995). Ovipositor steering mechanisms in braconid wasps. Journal of Hymenoptera Research 4, 110-120.

[46] QUicke DLJ (1991). Ovipositor mechanics of the braconine wasp genus Zaglyptogastra and the ichneumonid genus Pristomerus. Journal of Natural History 25, 971-977.

[47] Nash WA (1977). Strength of Materials: Theory and Problems (Schaum's Outline Series). McGrawHill Publishing Co., 2nd editio edn.

[48] Roesthuis RJ, Abayazid M, \& Misra S (2012). Mechanics-based model for predicting in-plane needle deflection with multiple bends. 2012 4th IEEE RAS \& EMBS International Conference on Biomedical Robotics and Biomechatronics (BioRob) 69-74.

[49] Sмith EL (1970). Evolutionary morphology of the external insect genitalia. 2. Hymenoptera. Annals of the Entomological Society of America 63, 1-27.

[50] Compton S \& Nefdt R (1988). Extra-long ovipositors in chalcid wasps: some examples and observations. Antenna 12, 102-105.

[51] Zhen WQ, Huang Dw, XIAO JH, \& YANg Dr (2005). Ovipositor length of three Apocrypta species: effect on oviposition behavior and correlation with syconial thickness. Phytoparasitica 33, 113-120.

[52] Peters RS, Krogmann L, Mayer C, Donath A, Gunkel S, Meusemann K, Kozlov A, Podsiadlowski L, Petersen M, Lanfear R, Diez PA, Heraty J, KJer KM, Klopfstein S, Meier R, Polidori C, Thomas S, Shanlin L, Zhou X, Wappler T, Rust J, Misof B, \& Niehus O (2017). Evolutionary history of the Hymenoptera. Current Biology 27, 1013-1018.

[53] Sмітн EL (1969). Evolutionary morphology of external insect genitalia. 1. Origin and relationships to other appendages. Annals of the Entomological Society of America 62, 1051-1079.

[54] Sмiтн EL (1972). Biosystematics and morphology of Symphyta - III external genitalia of Euura (Hymenoptera: Tenthredinidae): sclerites, sensilla, musculature, development and oviposition behaviour. International Journal of Insect Morphology and Embryology 1, 321-65.

[55] NÉnON JP, KACEM N, \& LANNIC LJ (1997). Structure, sensory equipment, and secretions of the ovipositor in a giant species of Hymenoptera: Megrahyssa atrata F. (Ichneumonidae, Pimplinae). The Canadian Entomologist 129, 789-799.

[56] Vilhelmsen L (2003). Flexible ovipositor sheaths in parasitoid Hymenoptera (Insecta). Arthropod Structure E Development 32, 277-287.

[57] Ahmed T, Zhang Tt, He KL, BAi Sx, \& WANG Zy (2013). Sense organs on the ovipositor of Macrocentrus cingulum Brischke (Hymenoptera: Braconidae): their probable role in stinging, oviposition and host selection process. Journal of Asia-Pacific Entomology 16, 343-348.

[58] WANG T, PAN L, ZHANG Y, \& DAI W (2015). Morphology of the mouthparts of the spittlebug Philagra albinotata Uhler (Hemiptera: Cercopoidea: Aphrophoridae). Arthropod Structure E Development 44, 121-130.

[59] KRenN HW \& Aspöck H (2012). Form, function and evolution of the mouthparts of blood-feeding Arthropoda. Arthropod Structure E Development 41, 101-118.

[60] Hamilton K (1981). Morphology and evolution of the rhynchotan head (Insecta: Hemiptera, Homoptera). The Canadian Entomologist 113, 953-974.

[61] Pollard DG (1972). The stylet structure of a leafhopper (Eupteryx melissae Curtis: Homoptera, Cicadellidae). Journal of Natural History 6, 261-271. 
[62] Rosell RC, Lichty JE, \& BRown JK (1995). Ultrastructure of the mouthparts of adult sweetpotato whitefly, Bemisia tabaci Gennadius (Homoptera: Aleyrodidae). International Journal of Insect Morphology and Embryology 24, 297-306.

[63] Auclair JL (1963). Aphid feeding and nutrition. Annual Review of Entomology 8, 439-490.

[64] Zhao L, DAi W, Zhang C, \& Zhang Y (2010). Morphological characterization of the mouthparts of the vector leafhopper Psammotettix striatus (L.) (Hemiptera: Cicadellidae). Micron 41, 754-759.

[65] Novotny V \& WiLson MR (1997). Why are there no small species among xylem-sucking insects? Evolutionary Ecology 11, 419-437.

[66] Malone AM, Watson R, \& Pritchard J (2016). The spittlebug Philaenus spumarius feeds from mature xylem at the full hydraulic tension of the transpiration stream. The New Phytologist 143, 261-271.

[67] CoHen AC (1990). Feeding adaptations of some predaceous Hemiptera. Annals of the Entomological Society of America 83, 1215-1223.

[68] Wenk P, Lucic S, \& Betz O (2010). Functional anatomy of the hypopharynx and the salivary pump in the feeding apparatus of the assassin bug Rhodnius prolixus (Reduviidae, Heteroptera). Zoomorphology 129, 225-234.

[69] Fontaine A, Diouf I, Bakkali N, Missé D, Pagès F, Fusai T, Rogier C, \& Almeras L (2011). Implication of haematophagous arthropod salivary proteins in host-vector interactions. Parasites E Vectors 4, 187.

[70] СоввеN RH (1978). Evolutionary trends in heteroptera: Part II. Mouthpart-structures and feeding strategies. Wageningen, The Netherlands: H. Veenman \& Zonen B. V.

[71] Clements AN (1992). The biology of mosquitoes (Vol. 1): Development, nutrition and reproduction. Chapman \& Hall.

[72] Wahid I, Sunahara T, \& Mogi M (2003). Maxillae and mandibles of male mosquitoes and female autogenous mosquitoes (Diptera: Culicidae). Journal of Medical Entomology 40, 150-158.

[73] TAWFIK MS (1968). Feeding mechanisms and the forces involved in some blood-sucking insects. Questiones entomolgicae 4, 92-111.

[74] Dimmoc G (1881). Anatomy of the mouth-parts and of the suctorial apparatus of Culex. Psyche 3, 231-241.

[75] SNodgrass RE (1959). Anatomical life of the mosquito. Smithsonian miscellaneous collections 139, $1-87$.

[76] Waldbauer GP (1962). The mouth parts of female Psorophora ciliata (Diptera, Culicidae) with a new interpretation of the functions of the labral muscles. Journal of Morphology 111, 201-215.

[77] Compton SG, Van Noort S, McLeish M, Deeble M, \& Stone V (2009). Sneaky African fig wasps that oviposit through holes drilled by other species. African Natural History 5, 9-15.

[78] Quicke DLJ, Wyeth P, Fawke JD, Basibuyuk HH, \& Vincent JFV (1998). Manganese and zinc in the ovipositors and mandibles of hymenopterous insects. Zoological Journal of the Linnean Society 124, 387-396.

[79] TuRRIsi GF \& Vilhelmsen L (2010). Into the wood and back: morphological adaptations to the wood boring parasitoid lifestyle in adult aulacid wasps (Hymenoptera: Aulacidae). Journal of Hymenoptera Research 19, 244-258.

[80] Weber H (1928). Zur vergleichenden Physiologie der Saugorgane der Hemipteren: Mit besonderer Berücksichtigung der Pflanzenläuse. Zeitschrift für vergleichende Physiologie 8, 145-186.

[81] Dai W, Pan L, Lu Y, Jin L, \& Zhang C (2014). External morphology of the mouthparts of the whitebacked planthopper Sogatella furcifera (Hemiptera: Delphacidae), with special reference to the sensilla. Micron 56, 8-16.

[82] Miles PW (1968). Insect secretions in plants. Annual Review of Phytopathology 6, 137-164.

[83] TJALLINGII WF \& Esch TH (1993). Fine structure of aphid stylet routes in plant tissues in correlation with EPG signals. Physiological Entomology 18, 317-328. 
[84] Will T, Furch ACU, Zimmermann MR, \& Wilkinson T (2013). How phloem-feeding insects face the challenge of phloem-located defenses. Frontiers in Plant Science 4, 1-12.

[85] Miles PW (1972). The Saliva of Hemiptera. Advances in Insect Physiology 9, 183-255.

[86] Ramasubramanian MK, Barham OM, \& Swaminathan V (2008). Mechanics of a mosquito bite with applications to microneedle design. Bioinspiration \& biomimetics 3, 046001 (10pp).

[87] Aoyagi S, Izumi H, \& FuKuda M (2008). Biodegradable polymer needle with various tip angles and consideration on insertion mechanism of mosquito's proboscis. Sensors and Actuators A: Physical $143,20-28$.

[88] Miles PW (1958). The stylet movements of a plant-sucking bug, Oncopletus fasciatus Dall. (Heteroptera: Lygeidae). Proceedings of Royal Entomological Society London. Series A, General Entomology 33, 15-20.

[89] KING PE (1962). The muscular structure of the ovipositor and its mode of function in Nasonia vitripennis (Walker) (Hymenoptera: Pteromalidae). Proceedings of Royal Entomological Society London. Series A, General Entomology 37, 121-128.

[90] Fergusson NDM (1988). A comparative study of the structures of phylogenetic importance of female genitalia of the Cynipoidea (Hymenoptera). Systematic Entomology 13, 13-30.

[91] Vilhelmsen L (2000). The ovipositor apparatus of basal Hymenoptera (Insecta): phylogenetic implications and functional morphology. Zoologica Scripta 29, 319-345.

[92] BENDER JC (1943). Anatomy and histology of the female reproductive organs of Habrobracon juglandis (Ashmead). Annals of the Entomological Society of America 36, 537-545.

[93] Lyngnes R (1960). Shape and function of the ovipositor in the three Hymenopterous species: Ephialtes extensor Thom. (Ichneumonidae), Spathius exarator L. (Braconidae), and Plutothrix coelius Walk. (Chalcididae). Norsk Entomologisk Tidsskrift 11, 122-134.

[94] Copland MJW (1976). Female reproductive system of the Aphelinidae (Hymenoptera: Chalcidoidea). International Journal of Insect Morphology and Embryology 5, 151-166.

[95] Leyva JL, Browning HW, \& Gilstrap FE (1991). Effect of host fruit species, size, and color on parasitization of Anastrepha ludens (Diptera: Tephritidae) by Diachasmimorpha longicaudata (Hymenoptera: Braconidae). Environmental Entomology 20, 1469-1474.

[96] MAcGIL EI (1946). The anatomy of the head and mouth-parts of Dysdercus intermedius Dist. Proceedings of the Zoological Society of London 117, 115-128.

[97] Pollard DG (1970). The mechanism of stylet movement in Psylla mali Schmidberger (Homoptera: Psyllidae). Zoological Journal of the Linnean Society 49, 295-307.

[98] Boyd DWJ, Cohen AC, \& Alverson DR (2002). Digestive enzymes and stylet morphology of Deraeocoris nebulosus (Hemiptera: Miridae), a predacious plant bug. Annals of the Entomological Society of America 95, 395-401.

[99] Oten KLF, Cohen AC, \& HAIn FP (2014). Stylet bundle morphology and trophically related enzymes of the hemlock woolly adelgid (Hemiptera: Adelgidae). Annals of the Entomological Society of America 107, 680-690.

[100] GRIFFITHS RB \& GoRdon RM (1952). An apparatus which enables the process of feeding by mosquitoes to be observed in the tissues of a live rodent; together with an account of the ejection of saliva and its significance in malaria. Annals of tropical medicine and parasitology 46, 311-319.

[101] Kong X \& WU C (2009). Measurement and prediction of insertion force for the mosquito fascicle penetrating into human skin. Journal of Bionic Engineering 6, 143-152.

[102] Aoyagi S, Izumi H, \& Fukuda M (2007). Biodegradable polymer needle with various tip angles and effect of vibration and surface tension on easy insertion. 2007 IEEE 20th International Conference on Micro Electro Mechanical Systems (MEMS) 397-400.

[103] Elias LG, Teixeira SP, Kjellberg F, \& Santinelo Pereira RA (2012). Diversification in the use of resources by Idarnes species: bypassing functional constraints in the fig-fig wasp interaction. Biological Journal of the Linnean Society 106, 114-122. 
[104] Gerling D, Quicke DLJ, \& Orion T (1998). Oviposition mechanisms in the whitefly parasitoids Encarsia transvena and Eretmocerus mundus. BioControl 43, 289-297.

[105] Belshaw R, Grafen A, \& Quicke DLJ (2003). Inferring life history from ovipositor morphology in parasitoid wasps using phylogenetic regression and discriminant analysis. Zoological Journal of the Linnean Society 139, 213-228.

[106] Alves TJS, Wanderly-Teixeira V, Teixeria AAC, Silva-Torres CSA, Malaquias JB, Pereira BF, Caetano FH, \& Cunha FM (2014). Parasitoid-host interaction: sensory structures involved in the parasitism behavior of Bracon vulgaris (Hymenoptera: Braconidae). Animal Biology 64, 365-381.

[107] Ko SY, Davies BL, \& Rodriguez y BAenA F (2010). Two-dimensional needle steering with a "programmable bevel" inspired by nature: Modeling preliminaries. Intelligent Robots and Systems (IROS), 2010 IEEE/RSJ International Conference on 2319-2324.

[108] Juárez-Hernández P, Valdez-Carrasco J, Valdovinos-Ponce G, Mora-Aguilera JA, OteroColina G, Téliz-Ortiz D, Hernández-Castro E, Ramírez-Ramírez I, \& González-Hernández VA (2014). Leaf penetration pattern of Aulacaspis tubercularis (Hempitera: Diaspididae) stylet in mango. Florida Entomologist 97, 100-107.

[109] Pollard DG (1968). Stylet peneteation and feeding damage of Eupteryx melissae Curtis (Hemiptera, Cicadellidae) on sage. Bulletin of Entomological Research 58, 55-71.

[110] Gauld ID, Bolton B, Huddleston T, Fitton MG, Shaw MR, Noyes JS, Day MC, Else GR, FerGUSSON NMD, \& WARD SL (1988). The Hymenoptera. Oxford University Press, British Museum.

[111] Rahman MH, Fitton MG, \& Quicke DLJ (1998). Ovipositor internal microsculpture in the Braconidae (Insecta, Hymenoptera). Zoologica Scripta 27, 319-331.

[112] Dweck HKM, Gadallah NS, \& Darwish E (2008). Structure and sensory equipment of the ovipositor of Habrobracon hebetor (Say) (Hymenoptera: Braconidae). Micron 39, 1255-1261.

[113] Fulton BB (1932). Notes on Habrocytus cerealellae, parasite of the Angoumois grain moth. Annals of the Entomological Society of America 26, 536-553.

[114] Whiting AR (1967). The biology of the parasitic wasp Mormoniella vitripennis (= Nasonia Brevicornis) (Walker). The Quarterly Review of Biology 42, 334-406.

[115] Austin AD \& Browning TO (1981). A mechanism for movement of eggs along insect ovipositors. International Journal of Insect Morphology and Embryology 10, 93-108.

[116] Austin AD (1983). Morphology and mechanics of the ovipositor system of Ceratobaeus Ashmead (Hymenoptera: Scelionidae) and related genera. International Journal of Insect Morphology and Embryology 12, 139-155.

[117] BenNet-Clark HC \& BenNeT-CLARK BYHC (1963). Negative pressures produced in the pharyngeal pump of the blood-sucking bug, Rhodnius prolixus. Journal of Experimental Biology 40, $223-229$.

[118] KIM W (2013). Mechanics of xylem sap drinking. Biomedical Engineering Letters 3, 144-148.

[119] Pompon J, Quiring D, Goyer C, Giordanengo P, \& Pelletier Y (2011). A phloem-sap feeder mixes phloem and xylem sap to regulate osmotic potential. Journal of Insect Physiology 57, 13171322.

[120] Chiten S (1970). Shear dependence of effective cell volume as a determinant of blood viscosity. Science 168, 977-979.

[121] Wells R \& Merrill E (1962). Influence of flow properties of blood upon viscosity-hematocrit relationships. Journal of Clinical Investigation 41, 1591-1598.

[122] Miles PW (1999). Aphid saliva. Biological Reviews 74, 41-85.

[123] Michael R (1989). The significance of pectinase in plant penetration by aphids. Canadian Journal of Zoology 17, 294-306.

[124] Kim BH, SEo ES, Lim JH, \& LeE SJ (2012). Synchrotron X-ray microscopic computed tomography of the pump system of a female mosquito. Microscopy Research and Technique 75, 1051-1058.

[125] KiкUсні K \& Mochizuкi O (2011). Micro-PIV (micro particle image velocimetry) visualization of red blood cells (RBCs) sucked by a female mosquito. Measurement Science and Technology 064002, 064002 (9pp). 
[126] Ribeiro JMC, Charlab R, \& Valenzuela JG (2001). The salivary adenosine deaminase activity of the mosquitoes Culex quinquefasciatus and Aedes aegypti. Journal of Experimental Biology 204, 20012010.

[127] MAcGregor ME (1931). The nutrition of adult mosquitoes: preliminary contribution. Transactions of the Royal Society of Tropical Medicine and Hygene 24, 465-472.

[128] Brożeк J \& HerczeK A (2004). Internal structure of the mouthparts of true bugs (Hemiptera, Heteroptera). Polish Journal of Entomology 73, 79-106.

[129] Garzo E, Bonani JP, Lopes JRS, \& Fereres A (2012). Morphological description of the mouthparts of the Asian citrus psyllid, Diaphorina citri Kuwayama (Hemiptera: Psyllidae). Arthropod Structure E Development 41, 79-86.

[130] Gussekloo SWS, Heinen R, \& Cerkvenik U (2016). Adaptations to substrate properties in the ovipositors of parasitic wasps (Ichneumonoidea). Integrative and Comparative Biology 56, E296-E296.

[131] Quicke DLJ (2015). The braconid and ichenumonid parasitoid wasps: biology, systematics, evolution and ecology. John Wiley \& Sons, Ltd, first edn.

[132] Buffington ML (2007). The occurrence and phylogenetic implications of the ovipositor clip within the Figitidae (Insecta: Hymenoptera: Cynipoidea). Journal of Natural History 41, 2267-2282.

[133] Polidori C, García AJ, \& Nieves-Aldrey JL (2013). Breaking up the wall: metal-enrichment in ovipositors, but not in mandibles, co-varies with substrate hardness in gall-wasps and their associates. PloS one 8, e70529.

[134] Elias LG, Kjellberg F, Antoniolli Farache HF, Almeida EAB, Rasplus Jy, Cruaud A, Peng YQ, YANG DR, \& Pereira SANTInelo AR (2018). Ovipositor morphology correlates with life history evolution in agaonid fig wasps. Acta Oecologica 90, 109-116.

[135] LeE RM \& Craig DA (1983). Maxillary, mandibulary and hypopharyngeal stylets of female mosquitoes (Diptera: Culicidae); a scanning electron microscope study. The Canadian Entomologist 115, 1503-1512.

[136] Angelini DR \& Kaufman TC (2004). Functional analyses in the hemipteran Oncopeltus fasciatus reveal conserved and derived aspects of appendage patterning in insects. Developmental Biology 271, 306-321.

[137] Anderson WG, Heng-Moss TM, Baxendale FP, Baird LM, Sarath G, \& Higley L (2006). Chinch bug (Hemiptera: Blissidae) mouthpart morphology, probing frequencies, and locations on resistant and susceptible germplasm. Journal of economic entomology 99, 212-221.

[138] Foster WA (1995). Mosquito sugar feeding and reproductive energetics. Annual Review of Entomology 40, 443-474.

[139] Schneider A, Frasson L, Parittotokkaporn T, Rodriguez y Baena FM, Davies BL, \& HuQ SE (2009). Biomimetic microtexturing for neurosurgical probe surfaces to influence tribological characteristics during tissue penetration. Microelectronic Engineering 86, 1515-1517.

[140] Pollard DG (1971). The use of polyporus for the investigation of stylet behaviour in the Hemiptera. Entomologia Experimentalis et Applicata 14, 283-296.

[141] Quicke DLJ \& FitTon MG (1995). Ovipositor steering mechanisms in parasitic wasps of the families Gasteruptiidae and Aulacidae (Hymenoptera). Proceedings of The Royal Society: Biological Sciences 261, 98-103.

[142] BLACKWELl J \& WeIH MA (1980). Structure of chitin-protein complexes: ovipositor of the ichneumon fly Megarhyssa. Journal of Molecular Biology 137, 49-60.

[143] Matsumura Y, Kovalev AE, \& Gorb SN (2017). Penetration mechanics of a beetle intromittent organ with bending stiffness gradient and a soft tip. Science Advances 3, eaao5469.

[144] Politi Y, Priewasser M, Pippel E, Zaslansky P, Hartmann J, Siegel S, Li C, Barth FG, \& Fratzl P (2012). A spider's fang: how to design an injection needle using chitin-based composite material. Advanced Functional Materials 22, 2519-2528. 
[145] Bano S, Ko SY, Rodriguez F, \& Rodriguez Y Baena F (2012). Smooth path planning for a biologically-inspired neurosurgical probe. Engineering in Medicine and Biology Society (EMBC), 2012 Annual International Conference of the IEEE 920-923.

[146] Leibinger A, Oldfield MJ, Rodriguez Y Baena F, Rodriguez F, \& Rodriguez Y Baena F (2016). Minimally disruptive needle insertion: a biologically inspired solution. Interface Focus 6, 20150107.

[147] Scali M, Pusch TP, Breedveld P, \& Dodou D (2017). Needle-like instruments for steering through solid organs: A review of the scientific and patent literature. Proceedings of the Institution of Mechanical Engineers, Part H: Journal of Engineering in Medicine 231, 250-265.

[148] Caborni C, Ko SY, De Momi E, Ferrigno G, \& Rodriguez y Baena F (2012). Risk-based path planning for a steerable flexible probe for neurosurgical intervention. 2012 4th IEEE RAS E EMBS International Conference on Biomedical Robotics and Biomechatronics (BioRob) 866-871.

[149] Moreira P, Patil S, Alterovitz R, \& Misra S (2014). Needle steering in biological tissue using ultrasound-based online curvature estimation. 2014 IEEE International Conference on Robotics and Automation (ICRA) 4368-4373.

[150] Frasson L, Ko SY, Turner A, Parittotokkaporn T, Vincent JF, \& Rodriguez y Baena F (2010). STING: a soft-tissue intervention and neurosurgical guide to access deep brain lesions through curved trajectories. Proceedings of the Institution of Mechanical Engineers, Part H: Journal of Engineering in Medicine 224, 775-788.

[151] Parittotokkaporn T, Frasson L, Schneider A, Davies BL, DegenaAr P, \& Rodriguez Y Baena F (2010). Insertion experiments of a biologically inspired microtextured and multi-part probe based on reciprocal motion. Engineering in Medicine and Biology Society (EMBC), 2010 Annual International Conference of the IEEE 2010, 3190-3193. 


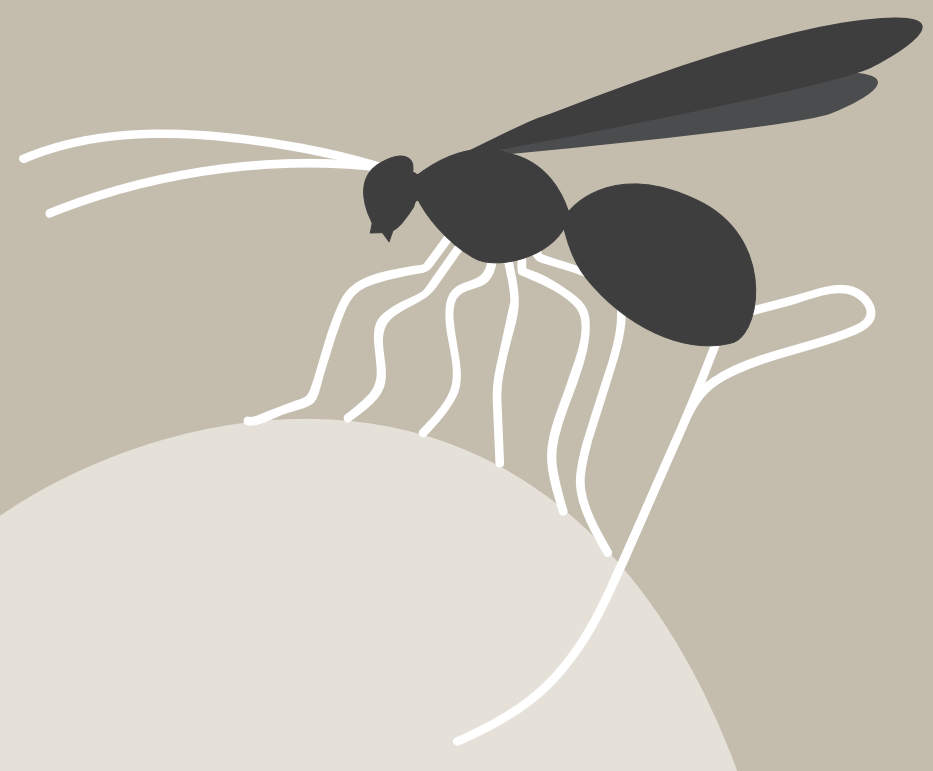




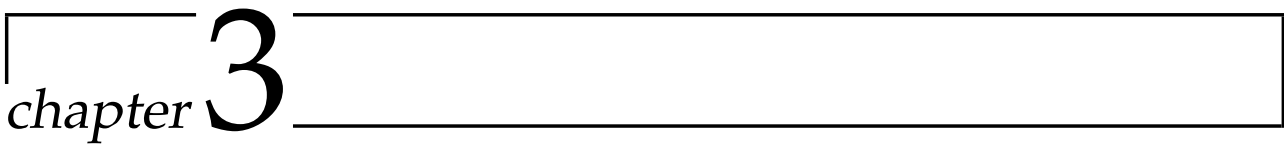

\section{Mechanisms of ovipositor insertion and steering of a parasitic wasp}

Uroš Cerkvenik $^{1}$, Bram van de Straat ${ }^{1}$, Sander W.S. Gussekloo ${ }^{1}$, and Johan L. van Leeuwen ${ }^{1}$

${ }^{1}$ Experimental Zoology Group, Department of Animal Sciences,

Wageningen University, De Elst 1, 6708 WD Wageningen, The Netherlands

Published as: Cerkvenik U, et al. (2017) Mechanisms of ovipositor insertion and steering of a parasitic wasp. Proceedings of the National Academy of Sciences of the United States of America. doi:10.1073/pnas.1706162114 


\section{Abstract}

Drilling into solid substrates with slender beam-like structures is a mechanical challenge, but is regularly done by female parasitic wasps. The wasp inserts her ovipositor into solid substrates to deposit eggs in hosts, and even seems capable of steering the ovipositor while drilling. The ovipositor generally consists of three longitudinally connected valves that can slide along each other. Alternative valve movements have been hypothesized to be involved in ovipositor damage avoidance and steering during drilling. However, none of the hypotheses have been tested in vivo. We used 3D and 2D motion analysis to quantify the probing behavior of the fruit-fly parasitoid Diachasmimorpha longicaudata (Braconidae) at the levels of the ovipositor and its individual valves. We show that the wasps can steer and curve their ovipositors in any direction relative to their body axis. In a soft substrate, the ovipositors can be inserted without reciprocal motion of the valves. In a stiff substrate, such motions were always observed. This is in agreement with the damage avoidance hypothesis of insertion, as they presumably limit the overall net pushing force. Steering can be achieved by varying the asymmetry of the distal part of the ovipositor by protracting one valve set with respect to the other. Tip asymmetry is enhanced by curving of ventral elements in the absence of an opposing force, possibly due to pretension. Our findings deepen the knowledge of the functioning and evolution of the ovipositor in hymenopterans and may help to improve man-made steerable probes.

\section{Significance}

Using slender probes to drill through solids is challenging, but desirable, due to minimal disturbances of the substrate. Parasitic wasps drill into solid substrates and lay eggs in hosts hidden within using slender probes and are therefore a good model for studying mechanical challenges associated with this process. We show that wasps are able to probe in any direction with respect to their body orientation and use two methods of insertion. One of the methods implies a minimal net pushing force during drilling. Steering was achieved by adjusting the asymmetry of the probe's distal end. Knowledge on probing mechanisms of wasps is important for the understanding of the hymenopteran evolution and for the development of minimally invasive steerable probes. 


\section{Introduction}

From a mechanical perspective, it is very difficult to drill into a solid substrate with a very thin probe, because it can easily bend and break. Parasitic wasps, however, do this regularly when they use their slender ovipositors to search for hosts in solid substrates, such as fruits or even wood. ${ }^{1-3}$

The general morphology of the ovipositor is similar across all wasp species ${ }^{4,5}$; it consists of four elements, called valves, of which two are often merged such that three functional valves remain (Fig. 1). In most species, the distal part of the ovipositor is morphologically distinct, ${ }^{3,6}$ which we will refer to as the tip. The valves can slide along each other ${ }^{5,7}$ and do not get dislocated under natural conditions, because they are longitudinally connected via a tongue-and-groove mechanism. ${ }^{5,8-10}$ The ovipositor and the 'wasp waist,' a constriction of the body between the first and second abdominal segment, ${ }^{11}$ are essential in probing behaviour and are therefore considered to be instrumental in the evolution of the order. ${ }^{11-15}$ The shape, structure, and mechanical properties of the ovipositors are putatively adapted to the substrates into which the animals need to probe, ${ }^{6,16-18}$ and because both substrates and hosts are so diverse, this might have resulted in high species diversification of the hymenopterans. ${ }^{13,14}$ However, to understand the observed diversity in the ovipositor shapes, understanding of the probing mechanics is essential.

Wasps are faced with two problems when searching for hosts in (solid) substrates: (i) how to insert the ovipositor without buckling/breaking it and (ii) how to maneuver with the ovipositor to reach the target.

Buckling is a mechanical failure of a structure which occurs, for instance, when a beam cannot withstand the applied axial load and bends, possibly beyond its breaking point. As buckling occurs more easily in slender beams, this is a real danger for parasitic wasps. Buckling depends on four parameters: $(i)$ the axial load applied on the beam, (ii) the second moment of area of the beam, (iii) how well is the beam fixed on both ends (i.e., 'free to slide sideways,' 'hinged,' or 'fixed'), and (iv) the length of the beam.

During puncturing, axial loading of the ovipositor cannot be avoided, so only the other factors can be adjusted. The second moment of area is largely determined by the diameter of the ovipositor and its wall thickness. To simplify insertion, the ovipositor must be as thin as possible, while the internal channel needs to be big enough for an egg to pass. Both of these requirements increase the chance of buckling. In all wasps, the ovipositor is fixed internally to the reproductive system and the muscles that move the ovipositor, ${ }^{4,19}$ so very little variance can be expected 

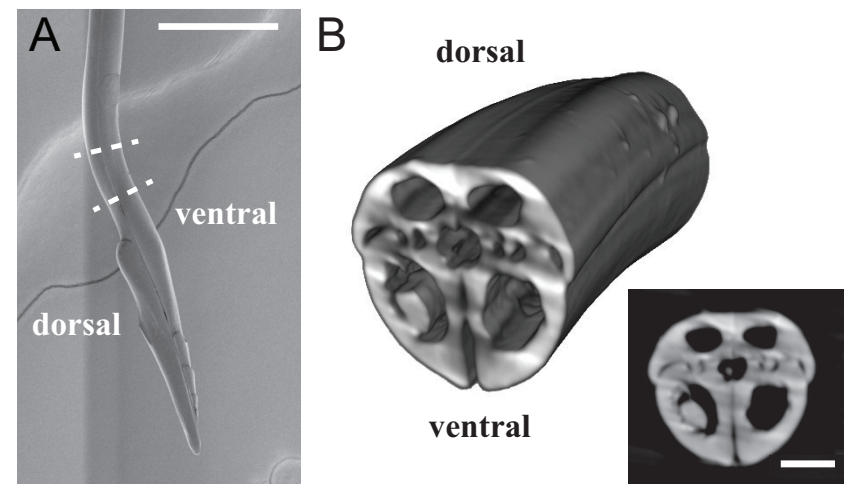

Fig. 1. Ovipositor of D. longicaudata. (A) SEM image of the ovipositor; side view. Region shown in B is indicated with dashed lines. (B) A $3 \mathrm{D}$ reconstruction of a part of the ovipositor obtained with a $\mu \mathrm{CT}$ scan. (B, Inset) Cross-section of the ovipositor showing the three valves. Scale bar: $100 \mu \mathrm{m}(\mathrm{A})$ and $10 \mu \mathrm{m}$ (B, Inset).

related to the fixation of the ovipositor. A parameter that can be changed is the 'functional' length of the ovipositor. Some wasps protrude only a small part of the total ovipositor outside their bodies before puncturing the substrate. The part retained in the abdomen is then either strongly coiled or telescopically retracted. ${ }^{20,21}$ In other species, the functional length of the ovipositor is reduced by supporting it by clamping the ovipositor with parts of their hind legs $11,18,22$ or with specialized sheaths. ${ }^{1,10,23,24}$

Little is known about the mechanisms parasitic wasps use for further insertion and buckling prevention of the ovipositor after the initial puncturing of the substrate. Vincent and King hypothesized a mechanism that wasps might use based on the morphology of the ovipositor (Fig. 2A). ${ }^{23}$ In the proposed mechanism, wasps apply a pulling force on two of the three valves, which are kept stationary because of the hook-like structures on their tips function as anchors. These valves serve as guides for the third valve that is pushed inward. According to the hypothesis, buckling of the protracted valve is avoided by limiting the amplitudes of forward motion. By alternating the protraction and retraction of the valves, the ovipositor is further inserted into the substrate, while avoiding excessive net push forces and axial loads that could damage the ovipositor. ${ }^{23}$

The second challenge during oviposition is that the wasps need to steer the ovipositor tip in the direction of the desired target. ${ }^{28}$ To do so, the ovipositor needs flexibility and a steering/bending mechanism that adjusts the tip direction during probing. Proposed bending mechanisms can be divided into passive and active ones (Fig. 2B-E). Passive bending originates from mechanical interactions of the inserted ovipositor with the substrate. Active bending occurs when bending moments originate from the relative movements of the ovipositor valves. 


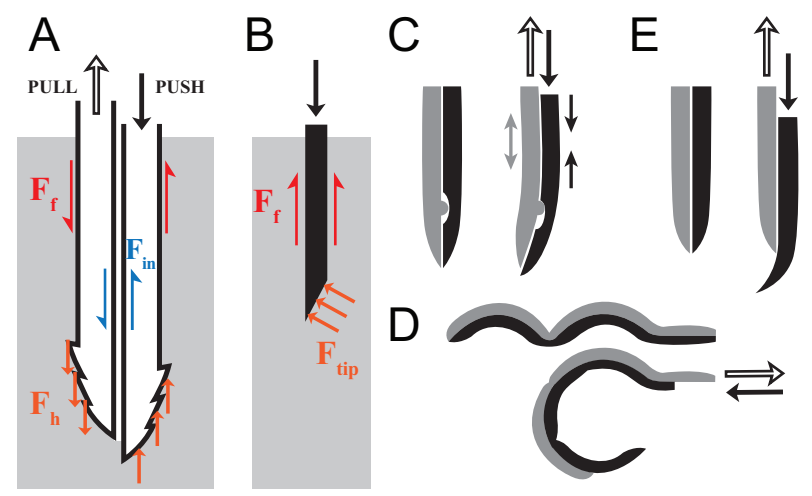

Fig. 2. Hypothesised insertion and steering mechanisms in 2D. Full arrows represent push forces and empty arrows pull forces. (A) The push-pull mechanisms ${ }^{23}$ (only two valves are shown for clarity). Inner friction $\left(\mathbf{F}_{\mathbf{i n}}\right)$ is considered negligible. Friction along the shaft $\left(\mathbf{F}_{\mathbf{f}}\right)$ of the two pulling valves, together with the hook forces $\left(\mathbf{F}_{\mathbf{h}}\right)$, keep the ovipositor anchored in the substrate and counteract the friction and hook (cutting) forces of the pushing valve (modified from ref. [23]). (B) A bevel shape of a needle leads to bending due to asymmetrical tip forces $\left(\mathbf{F}_{\text {tip }}\right)$. (C) Restriction in inter-element displacements ${ }^{25}$ causes bending due to tensile (gray arrows) and compression (small black arrows) forces (modified from ref. [25]). (D) Arched ovipositors bend due to differential sclerotization of valve segments ${ }^{26}$; see text (modified from ref. [26]). (E) Pretension of individual elements ${ }^{27}$ leads to incurving upon their protraction as observed in hemipteran mouthparts (modified from ref. [27]).

Passive bending presumably occurs when an ovipositor has an asymmetric beveled tip (Fig. 2B). The asymmetric forces acting on such a tip push the tip away from a straight path. ${ }^{29,30}$ Rotation of the bevel can be used to adjust the tip direction during insertion. ${ }^{31}$ The tips of most ovipositors across species are asymmetric ${ }^{6,32,33}$ and can thus potentially function as a bevel. The bevel shape can presumably be enhanced by changing the relative positions of the valves. An adjustable bevel may control the degree of bending, similar to what has been proposed for a new generation of steerable needles. ${ }^{34}$

Three mechanisms have been proposed for active bending. In the first mechanism, special anatomical structures limit the motion range of individual valves. ${ }^{25,35}$ Bending occurs due to tension and compression in individual valves (Fig. 2C). A second active bending mechanism relies on differences in valve sclerotization. ${ }^{26}$ The distal part of ovipositors relying on this bending mechanism consists of heavily sclerotized, stiff arches, alternated with less sclerotized and flexible nodes. At rest, the arches and nodes of the dorsal and ventral valves are aligned and the ovipositor is approximately straight. When the ventral or dorsal valves are protracted, the arches align with nodes, which leads to bending (Fig. 2D). The third possible mechanism of active bending has been hypothesized for the control of hemipteran mouthparts. Similar to the ovipositors, the hemipteran mouthparts consist of multiple slender elements that are interconnected longitudinally and are able to slide along each other. It is assumed that bending moments in hemipteran mouthparts originate 
from (pre)tension of the elements. ${ }^{27,36}$ The elements possess a certain level of inner tension and tend to curve to one side when not opposed. At rest, the elements are aligned with their tips so they counteract each other, resulting in a straight structure. When an individual element protracts, its tip curves inward toward the other elements (Fig. 2E). In all three mechanisms, the amplitude of the protraction and retraction of the valves probably correlates with the amount of bending and offers a way to control the curvature of the ovipositor during insertion.

Despite the proposed importance of ovipositors for the evolutionary success of hymenopterans, there is only a small number of empirical studies quantifying mechanical properties of the ovipositors. ${ }^{6,16-18}$ The proposed theories of probing are based on morphological data, with only a few studies focusing on the ovipositor inside the substrate, ${ }^{28,37}$ but no one has ever analysed the dynamics of probing inside the substrate.In this work, we aim to quantify the ovipositor use (range, speed, and curvature of probing) in relation to substrate density and to determine which of the proposed methods of insertion and steering are used by parasitic wasps. We do this using the species Diachasmimorpha longicaudata, which provides an excellent example because of its long and slender ovipositor. Extrapolation of our results will also provide insight into probing and steering possibilities of other groups of parasitic hymenopterans and possibly of hemipterans and mosquitoes, as they use similar structures to probe for food. In addition, our study will add to the understanding of the functional demands acting on the ovipositor and the mechanism for drilling with slender probes. This, in turn, can be applied in the development of man-made instruments for tunnelling, insertion, or probing. Such knowledge will presumably also help in the development of novel steerable surgical tools. ${ }^{38-43}$

\section{Results}

We presented 28 wasps with two different gel densities and stiffnesses (parameters presented in Table 1 and Fig. S1). For details on calculations of gel parameters see SI Materials and methods. Three wasps did not probe in both substrates. We analyzed only instances where wasps inserted $>60 \%$ of their ovipositor inside the substrate. For three of the animals, the top camera recording their orientation during probing stopped working. Their data were excluded from the calculations of the range of probing, but were included in the velocity analysis. This amounted to 107 and 92 insertions used for range calculations, and 117 and 113 insertions for speed calculations in $2 \%$ and $4 \%$ gels, respectively. 
Table 1: Gel parameters. Storage $\left(G^{\prime}\right)$, loss $\left(G^{\prime \prime}\right)$, dynamic shear $\left(G^{*}\right)$, and elastic $\left(E^{*}\right)$ moduli.

\begin{tabular}{ccccr}
\hline Conc. & $\mathrm{G}^{\prime}, \mathrm{kPa}$ & $\mathrm{G}^{\prime \prime}, \mathrm{kPa}$ & $\mathrm{G}^{*}, \mathrm{kPa}$ & $E^{*}, \mathrm{kPa}$ \\
\hline $2 \%$ & 11.970 & 0.676 & 11.989 & 35.967 \\
$4 \%$ & 68.544 & 4.615 & 68.700 & 206.099 \\
\hline Con
\end{tabular}

Conc., concentration

\section{Description of probing process}

When starting to probe, an individual wasp lifted its abdomen, oriented the stillsheathed ovipositor vertically, and punctured the substrate with the most distal part of the ovipositor tip. While inserting the ovipositor deeper, the sheaths peeled away from the ovipositor base into a hairpin-like structure (Fig. 3A). Often, the wasp partially retracted the ovipositor within the substrate and reinserted it along a different trajectory. The wasp did not change its body orientation during an insertion session. A single insertion session contained 1-16 insertions (see Fig. 3B for a typical example of an insertion session). Individual insertions were not continuous, but consisted of minute retractions and reinsertions, especially when making curved insertions. The retractions were sometimes also used to make minor adjustments to the direction of insertion.

\section{Probing Range}

We calculated the maximum arc length of each insertion trajectory, as well as the radius $(\mathbf{r})$, depth $(\mathbf{d})$, and position vector $(\mathbf{R})$ of the respective endpoints. We took the trajectory insertion angle $\alpha$ (deviation from $90^{\circ}$ ) as the angle between the $\mathbf{R}$ and the vertical vector along the depth axis (Fig. 3B).

From a single horizontal body orientation, wasps were able to probe in all directions within the gel (Fig. 3C), and we observed no directional preference of insertions belonging to the same session (Fig. S2). No clear directional preference was seen in the combined behavior of all animals (Rayleigh test, $P=0.5$ and $P=0.05$ for $2 \%$ and $4 \%$ gels, respectively).

The correlation between the vertical and horizontal component of the position vector (Pearson's correlation $=0.57, P<0.001$ ) shows that a deep probe had a large chance of having a limited horizontal amplitude and vice versa (Fig. 3D).

In general, the probing space of the wasps can be visualized as a cone with a curved base. The radius of the cone is substrate-dependent and is larger in the $2 \%$ gel than in the $4 \%$ gel (respective medians: $1.90 \mathrm{~mm}$ and $1.19 \mathrm{~mm}$, Mann-Whitney U test, $P<0.001)$. 

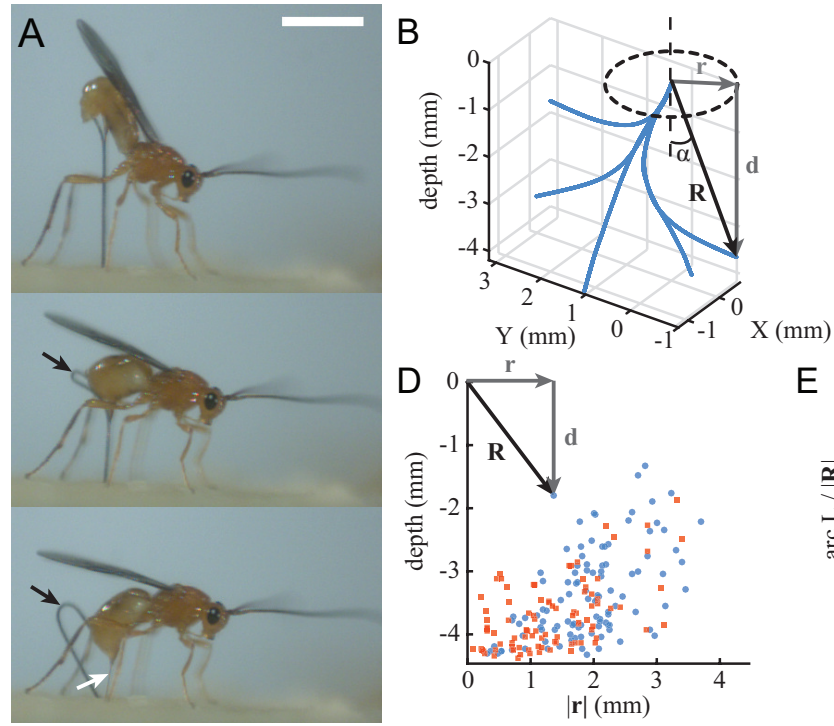

C
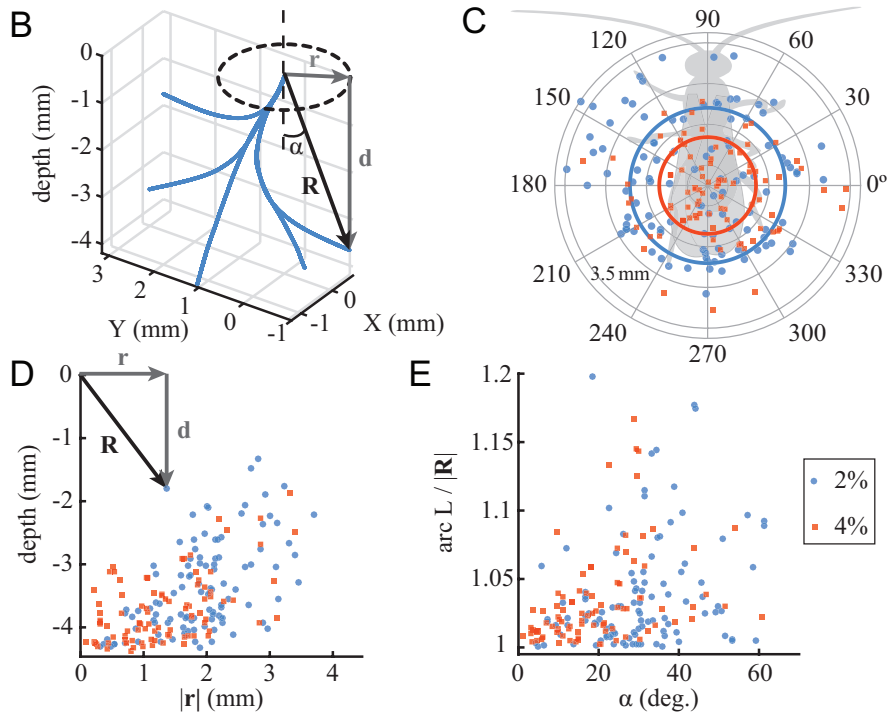

Fig. 3. Insertion behavior of probing wasps depends on substrate properties. (A) General probing behavior. The wasp positions its ovipositor vertically and punctures the substrate. The sheaths (black arrows) gradually detach and fold away from the ovipositor (white arrow) during deeper insertion. (Scale bar: $5 \mathrm{~mm}$.) (B) Example of a single insertion session in 3D with parameters used in the analysis: $\mathbf{R}$, position vector of the insertion trajectory endpoint; $\mathbf{r}$, radius of the endpoint; $\mathbf{d}$, depth vector; $\alpha$, insertion angle. (C) Horizontal probing range showing the radii of the endpoints, corrected for the animal orientation (silhouette). Colored circles indicate median values of $\mathbf{r}$ for different substrates. Blue, $2 \%$ gel; red, $4 \%$ gel. (D) Vertical probing range: depth of trajectories plotted against their respective radii. The depth is shallower with increasing radius. (E) Ratio of arc length and the magnitude of $\mathbf{R}$ indicates the deviation from a straight path (ratio close to 1 indicates straight insertions). In softer gels, wasps reach higher radii by inserting their ovipositors straight, but at acute angles.

Deviation from a straight path of the insertion can be estimated by taking the ratio of its arc length and the magnitude of its $\mathbf{R}$. Comparing the ratios against insertion angles, we see more insertions with high angles and with low bending in the $2 \%$ than in the $4 \%$ gel (Fig. 3E).

\section{Curvature and speed of insertions}

Wasps were able to strongly curve their ovipositors during probing with the maximal recorded values for curvature $(\kappa)$ up to $1.6 \mathrm{~mm}^{-1}$, or 0.048 dimensionless curvature ( $\kappa$ multiplied with the ovipositor width). Occasionally, we even captured complex insertion trajectories consisting of multiple bends in different directions. The majority of insertions, however, had very little curvature (Figs 3E and 4). Most parts of insertions were $<0.02$ dimensionless curvature, with the median values 0.0060 and 0.0069 for $2 \%$ and $4 \%$ gel, respectively. Only $\sim 1 \%$ of the parts of insertions had a dimensionless curvature $>0.03$. There was a slight, but significant, difference in curvature distribution between the $2 \%$ and $4 \%$ gels (Mann-Whitney $\mathrm{U}$ test, $P<0.001$ ), and we observed more insertions with dimensionless curvatures $>0.02$ in the denser gel. 


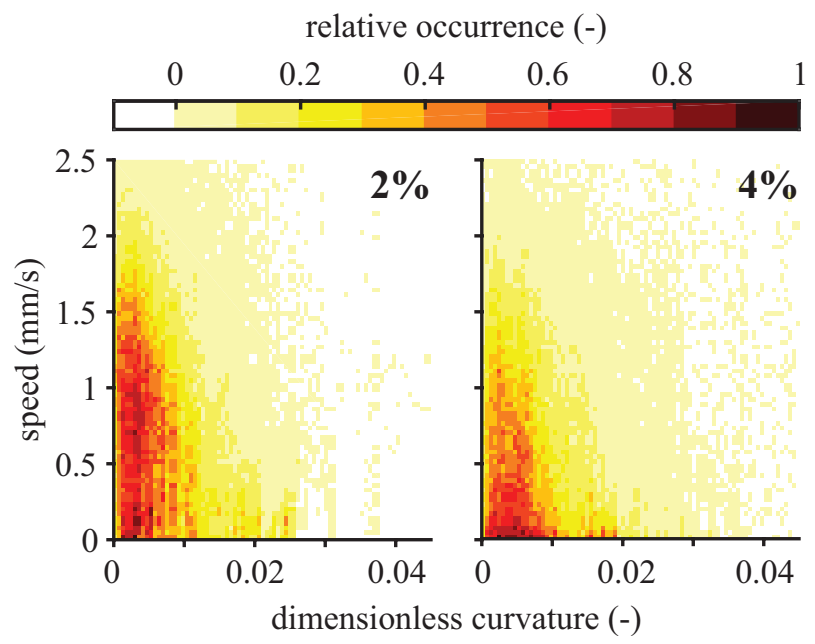

Fig. 4. Speed of probing decreases with increasing dimensionless curvature ( $\kappa$ multiplied with ovipositor diameter) and stiffness of the substrate. The heat maps show the relative frequency of specific speed and curvature combinations. Each axis has been divided into 80 bins of equal size, and the occurrence (number) of the points relative to the number of points in the most dense bin in the grid is presented as the color of the contours. The general pattern is hardly effected by the bin size (Fig. S6).

The ovipositor insertion was generally done at low speeds and accelerations (examples shown Figs S3-S5). Wasps probed faster in 2\% gel (Mann-Whitney U test, $P<0.001$ ), with median insertion speed: $0.73 \mathrm{~mm} \mathrm{~s}^{-1}$ in $2 \%$ gel and $0.55 \mathrm{~mm} \mathrm{~s}^{-1}$ in $4 \%$ gel. There is also a clear relation between curvature and insertion speed (Fig. 4 and Fig. S6). Insertions with high curvature were always done at low speed. The highest speeds were only observed at low curvatures, although the majority of insertions, even with low curvature, were done at speeds $<1.5 \mathrm{~mm} \mathrm{~s}^{-1}$.

\section{Changing tip morphology}

We observed clear changes in the asymmetry of the ovipositor tip during relative movements of the dorsal and ventral valves (Fig. 5). When the dorsal valve was retracted, the ventral valve(s) curved dorsally across the midline of the ovipositor. When the ventral valves were retracted, however, no curving of the dorsal valve tip was observed. Protraction of ventral valves can be also seen in peaks of the tip orientation graphs (red arrows in Fig. 6).

\section{Valve kinematics and steering}

Wasps used two methods of ovipositor insertion, which are presented in the three examples in Fig. 6 (see also Movies S1-S3). In the first method, the ovipositor was pushed into the substrate as a whole, with very little relative movement of 


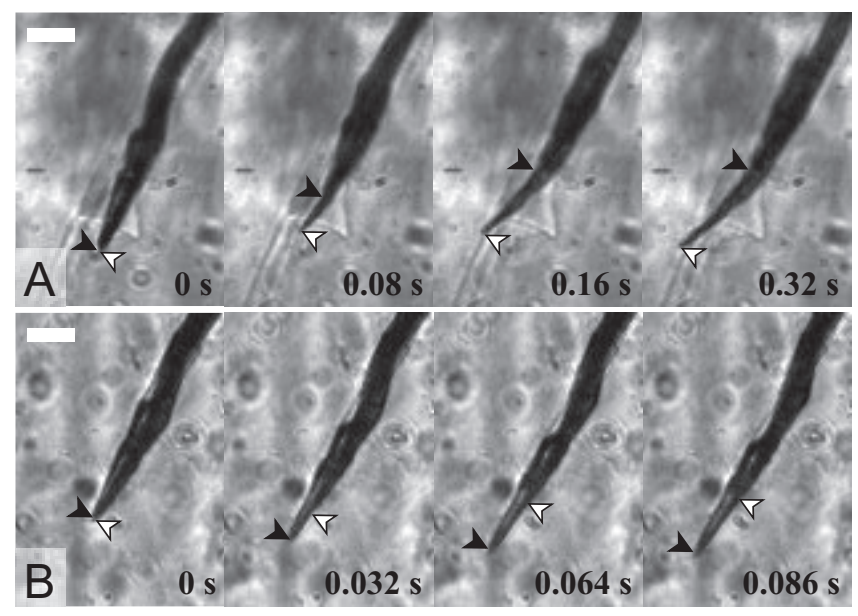

Fig. 5. Protraction of dorsal and ventral valves affect the shape of the ovipositor tip. (A) Protraction of the ventral valve(s) (white arrow) leads to the deformation and incurving of the ovipositor tip. (B) Protraction of the dorsal valve (black arrow) does not result in curving of the tip. (Scale bars: $50 \mu \mathrm{m}$.)

individual valves (pushing intervals noted in Fig. 6A and B). This method was only observed in the softer, $2 \%$ substrate, and we will refer to this method as the 'pushing method.' The second, more common, method involved clear alternating movements of the valves throughout the insertion process, which we will call the 'alternating method' (Fig. 6A and C).

Straight and curved paths were achieved by using both of these methods. Straight insertions using the pushing method were obtained by inserting the ovipositors with valves aligned or a slight protraction of the dorsal valve. In the alternating method, the valves were moved around a positive offset value (Fig. 6A and Fig. S1). Curved insertions were achieved by having the ventral valves protracted for an extended period. Minor changes in the negative valve offset were observed in the pushing method (Fig. 6B and Fig. S2), whereas the valves moved around a negative offset value in the alternating method (Fig. 6C and Fig. S3).

From the acceleration data, we calculated the net forces acting on the valves which were in the order of hundreds of piconewtons (SI Materials and methods and Figs S3-S5). 

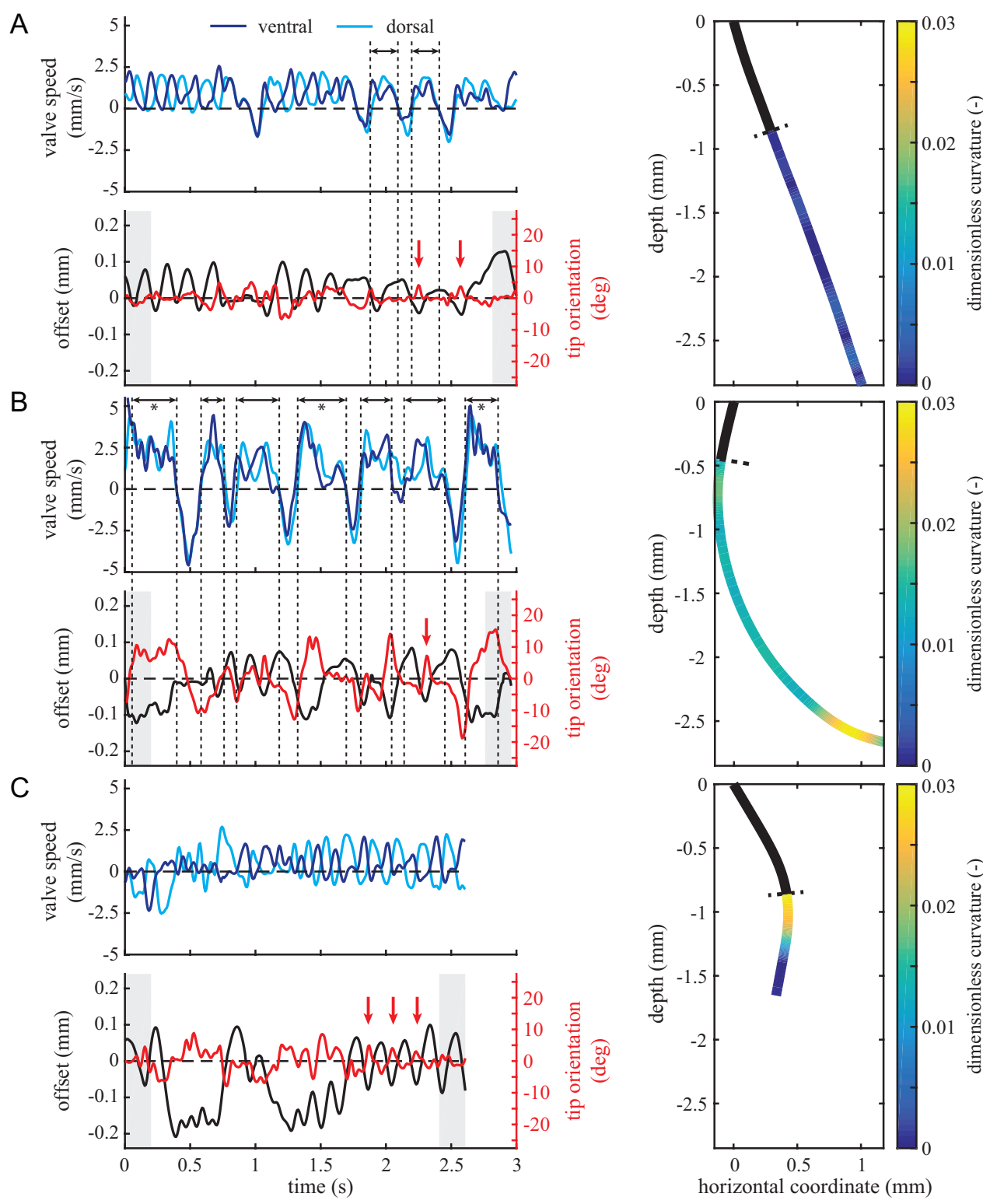

Fig. 6. Examples of ovipositor insertions. Shown are valve speeds, valve offset, tip orientation, and a 2D trajectory with its curvature (nontracked parts are shown in black). Shaded areas: less accurate calculations due to edge effects. Negative valve offset indicates protraction of ventral ${ }^{*}$ valve(s). (A) Example of a straight insertion in $2 \%$ gel showing both methods of insertion: alternating valves (valve speed 'out of phase') and pushing (valve speed 'in phase'). Intervals above the graphs indicate the pushing method of ovipositor insertion (curving indicated with asterisks), and red arrows indicate the small changes in tip orientation that occur even on short protractions of the ventral valves (in all graphs). (B) Example of a curved insertion in $2 \%$ gel using the pushing method (intervals) with small changes in valve offset. (C) Example of a curved insertion in $4 \%$ gel using the alternating valve method. In both (B) and $(C)$, ventral valves are protracted for a prolonged period, which leads to sustained changes in tip orientation, resulting in a curved trajectory.

"In the published version, this was erroneously stated as 'protraction of dorsal valve(s)'. 


\section{Discussion}

Parasitic wasps probe into substrates to deposit eggs in hosts hidden within. Therefore, they need to pierce and explore the substrate, ${ }^{3,6}$ locate the host, and sometimes also pierce through the host's integument, ${ }^{44-47}$ which is all done with the ovipositor apparatus only. Although the probing behaviour of parasitic wasps has been repeatedly mentioned in the literature, ${ }^{3,6,17,18,48-51}$ no quantitative studies have been performed until now. Here, we show evidence that wasps are able to explore a large space from a single puncture point and that they use relative valve motions to insert and steer the ovipositor.

Movements of the ovipositor inside the substrate are complex and originate from the interplay between substrate and relative movements of individual ovipositor valves.

Two methods of ovipositor insertion were used by the wasps: (i) pushing of the entire ovipositor with minimal relative valve movements and (ii) insertions with alternating valve movements of high amplitude. During pushing, the valves were moved together, and the shape of the ovipositor tip barely changed. In the second method, the valves moved alternatively, resulting in a continuously changing shape of ovipositor tip.

The pushing method was only used in the soft gel, indicating that it might only be applicable in low-resistance environments. The second method, using alternating valve movements, was observed in both gels, showing that this can also be used in high-resistance environments. This is in accordance with the mechanism proposed by Vincent and King, ${ }^{23}$ which predicts that alternating valve movement can be used for ovipositor insertion. According to the mechanism, some valves are being pulled at which anchors the ovipositor, allowing for the protraction of other valve(s). This predicted behaviour is clearly seen in our data. Similar alternating movements, albeit of slender mouthparts, ${ }^{52}$ have also been reported in feeding mosquitoes. When probing in the host's integument, the mandibles and maxillae oscillate along their longitudinal axes. ${ }^{53-56}$

Estimation of net forces on the valves (Figs S3-S5) revealed that they are very low (piconewton range), which is also in agreement with the prediction of Vincent and King. ${ }^{23}$ Low forces were also suggested to occur during puncturing ${ }^{55}$ and further insertion ${ }^{57}$ of a mosquito proboscis. The low force values are partially attributed to the oscillatory movements of individual elements. ${ }^{55,58}$ The forces estimated in this study are the sum of all forces acting on the ovipositor valves and can therefore not be directly compared with those in the mosquito experiments. The actual forces the valves exert on the substrate and friction forces within the substrate are probably 
substantially bigger. Unfortunately, it is currently not possible to obtain these forces in vivo. In addition, making accurate estimates of muscle forces is extremely difficult because of the small size of the animals. Our data nevertheless indicate that ovipositors can highly efficiently drill inside substrates, possibly with minimal net outside pushing force.

We observed that curved ovipositor trajectories were obtained using both insertion methods. The presence of multiple curves in single insertions indicates a degree of steering similar to that observed in previous studies on wasps. ${ }^{28,37}$ Furthermore, the insertion trajectory pattern in a probing session (Fig. 2B) and the multiple bends within a single insertion are similar to what has been observed for the probing of hemipterans. ${ }^{27,59,60}$ This indicates that wasps and hemipterans might use similar mechanisms of probing. Both tend to explore a wide range of the substrate from a single puncture point. Several hypotheses have been postulated about the mechanism of ovipositor steering in wasps. ${ }^{6,25,35}$ Our direct observations of the probing process revealed that steering is achieved by shape changes of the ovipositor tip (Fig. 5), creating various degrees of geometrical asymmetry. This is similar to the bevelled tip of hypodermic needles, which has been shown to induce asymmetric reaction forces from the substrate that result in bending. ${ }^{30,31,61,62}$ We observed three ways in which the wasp can create such an asymmetrical tip.

First, the morphology of the ovipositor tip is asymmetric (Fig. 1A), which might, by itself, result in bending effect, but this was rarely $(n=2)$ observed in our experiments. Second, by protracting one or more valves far beyond the other(s), the asymmetry of the tip is reinforced. This technique was observed during both insertion methods. In the third, most commonly observed, condition, the ventral valves are protracted to create a pronounced bevel. Protraction of the ventral valves (or retraction of the dorsal valve) caused the tip to bend toward the dorsal valve, probably because of the so-called preloading. Preloaded elements curved in a determined direction when no opposing force was present. The mechanism of preloaded elements has been proposed as a possible steering mechanism for hemipteran mouthparts. ${ }^{27}$ Although the incurving properties have been observed in mouthparts of dead hemipterans, ${ }^{36,63,64}$ they have never been observed in any species in vivo until now. Combining the second and the third point, one can expect that a bigger amplitude of ventral valve protraction will lead to stronger curvatures. This was, however, only partly confirmed in our study, as the amplitude of the negative offset does not always lead to a bigger change in tip orientation (Fig. 6). The inconsistency probably arises from the analysis of shadow images in which no distinction can be made between the two ventral valves, but which was necessary to increase the depth of focus of our recordings. 
Our results show that wasps can explore a large volume by using the insertion and steering mechanisms described above. Furthermore, the wasp can do this from a single body orientation, using a single puncture point. Puncturing is arguably the most difficult step in probing, and it is therefore beneficial for the wasp if the ovipositor can be steered in any direction from a single puncture point. However, steering mechanisms described above only explain bending in one direction. Considering that the animal does not change the position of the body, the only other possible explanation for the observed range is that the animals are capable of rotating the ovipositor or its tip within the substrate.

For our species, the range can be envisioned similarly to a cone with the height equal to the length of the ovipositor and a curved base. The radius of the cone is dependent on the substrate stiffness and is slightly larger in the soft substrate. Despite a larger radius in the soft gel, the majority of insertions were still nearly straight, including insertions with a large horizontal displacement. This may be because the wasps inserted their ovipositors at acute angles with respect to the substrate surface. Unfortunately, it was impossible to determine the angle between the ovipositor and the substrate at the time of puncturing with our camera setup. Puncturing at acute angles is only possible when forces are small, because large lateral forces might result in buckling. In soft substrates, these forces are smaller than in stiff ones, allowing the wasps to puncture at shallow angles. However, such an angular insertion limits the possibility to probe in the opposite direction, because that would require very high curvatures.

In the stiff gel, there is a larger need to position the ovipositor perpendicular to the surface to avoid large lateral forces that might result in buckling. Therefore, in stiff substrates, wasps need to use bending of the ovipositor to enlarge their probing space. Our data show that this is achieved by adjusting the curvature for which relative movements of the valves are crucial. This is probably enhanced by the stronger reaction forces experienced as a result of the stiffer gel.

We also showed that substrate density has a negative effect on insertion speed. Stiffness (Fig. S1) and failure stress increase with gel density, ${ }^{65,66}$ which makes penetration into denser substrates more energetically costly. The natural substrates the wasps probe into for hosts are, amongst others, citrus fruits, peaches, figs, and apples. ${ }^{67-69}$ It is impossible to visualize the probing process in such substrates, so we substituted them for translucent gellan gels of different densities. There are some important differences between the gels and the fruits, as the latter are anisotropic and composed of layers and fibres. The shear and elastic moduli (Fig. S1) of the softest gel used in this study were approximately two orders of magnitude smaller than the moduli reported for mature peaches ${ }^{70,71}$ and the peel of citrus fruits. ${ }^{72,73}$ The 
densest gel was only one order of magnitude softer than these fruits. The reported values for fruits are estimates of a healthy condition, and it is expected that they drastically decrease in fruits infested with fruit-fly larvae. Unfortunately, mechanical properties of the relevant decaying fruits are not known. It is thus impossible to determine whether the physical properties of the gels used in this study match those of the decaying fruit. Our estimation is that the gels represent such conditions reasonably well. Because we saw changes in probing kinematics in different gel densities, we hypothesize that wasps can adapt to different material properties in fruits.

Similar to increased substrate density, curving of the ovipositor also increased the amount of energy required for ovipositor insertion due to increased friction between the ovipositor and the substrate. This explains the lower insertion speeds for curved parts of trajectories observed in our study. It also indicates that curving is an energetically expensive behavior that might be better avoided if possible.

We show that reciprocal valve movements are used when inserting slender probes into solid substrates. The estimated net forces acting on the valves are the first quantification of the push-pull mechanism in vivo. The low values are in agreement with the proposed insertion mechanism characterized by minimal net external pushing force. Furthermore, the relative position of the valves dictates the shape of the tip and influences the direction of probe insertion, probably by manipulating the size and direction of the substrate reaction forces. This improves the insight in the overall mechanism of oviposition in hymenopterans. Understanding of the mechanism will deepen the knowledge of adaptations in the ovipositor apparatus and the evolution of the taxon as a whole.

In addition, our findings can help advance the development of steerable man-made probes that is on the rise in the past decade $30,62,74$ and is particularly relevant for the design of multi-element probes. ${ }^{39,40,75}$ Miniaturization of the needle diameter should presumably be possible also for the needles used in solid substrates if the individual elements are operated in an alternating manner. The adjustable bevel shape, although already partially implemented (e.g., ref. [61]), can be greatly enhanced by implementing pretension of the elements. Despite having multiple elements, the control of such needles may not necessarily be complicated, as it would be enough to monitor the juxtaposition of individual elements that would dictate the direction and amplitude of curving. 


\section{Materials and methods}

\section{Substrate properties}

We used gellan gel, a microbial polysaccharide, ${ }^{76}$ as the probing substrate because of its translucency, isotropic properties, and readiness of the animals to insert their ovipositors in it. The rheological properties, namely, the storage $\left(G^{\prime}\right)$ and loss $\left(G^{\prime \prime}\right)$ moduli, of the gels used in the experiments were measured with a rotational rheometer (Anton Paar) and a cone plate probe (diameter of $25 \mu \mathrm{m}$ ) at $20^{\circ} \mathrm{C}$. The gels were amorphous and considered isotropic (i.e., random cross-linking of hydrophilic polymer chains was assumed). To ensure a strong contact with the equipment, the gels were poured onto the base plate, and the probe was lowered into the gels while they were still warm. The gel was then left to cool down before starting the experiments. The linear strain region of the gel was obtained by first measuring the response to a changing strain (amplitude range: 0.1-100\%) at a constant angular velocity $\left(0.16 \mathrm{rad} \mathrm{s}^{-1}\right)$. Another gel of the same concentration was used for the measurements at a constant strain within the linear strain regime $(0.2 \%)$ with a changing angular frequency of the probe $\left(1-100 \mathrm{rad} \mathrm{s}^{-1}\right)$. The frequency measurements were repeated three times with 1-min intervals. The obtained moduli were averaged across repetitions and frequencies to calculate the dynamic shear modulus $\left(\mathrm{G}^{*}\right)$ and the dynamic elastic modulus $\left(E^{*}\right)$. We assumed a Poisson's ratio $(v)$ of 0.5 because of the high water content of the gels. See SI Materials and methods for details of the calculations.

\section{Animals}

Adult parasitic wasps (D. longicaudata) and their host, the Mediterranean fruit-fly Ceratitis capitata) were kept separately in rearing cages $(30 \mathrm{~cm} \times 30 \mathrm{~cm} \times 30 \mathrm{~cm}$, BugDorm; MegaView Science) at $24^{\circ} \mathrm{C}$ and 12/12-h light/dark cycle, with ad libitum access to water and food. Wasp were fed commercially available honey, and flies were fed a mixture of glucose and brewer's yeast dissolved in tap water. The flies could freely reproduce and lay their eggs in perforated plastic bottles. The eggs were harvested and put in oxygenated water for $1 \mathrm{~d}$ before transferring them to the larval medium, which was a mixture of brewer's yeast, carrot powder, sodium benzoate (food preservative), methylparaben (antifungal agent), hydrochloric acid ( $\mathrm{HCl}$; acidity regulator), and tap water. The larvae were exposed to parasitoids between the fourth and seventh day after hatching. After parasitation, the larvae were taken away from the wasps and left to develop and pupate in small boxes of vermiculite. After $\sim 2 \mathrm{wk}$, the wasps emerged from pupae of parasitized larvae. Fly 
larvae used for propagation of fly colonies were never exposed to parasitation and were kept apart from the wasps. For details of the rearing protocol, see SI Materials and methods.

\section{Experimental setup and data acquisition}

The 3D insertion path of the ovipositor in the substrate was recorded with two synchronized high-speed video cameras (Fastcam SA-X2; Photron) fitted with macro lenses (MP-E 65; Canon; $5 \times$ magnification factor) at $125 \mathrm{fps}$. The cameras' optical axes were perpendicular to each other $\left(89^{\circ} \pm 1^{\circ}\right.$; Fig. 7A). Two near-collimated light beams were produced by using combinations of an approximate point light source and a 4-diopter lens, arranged in a backlight configuration. This resulted in a shadow image and a depth of focus of $\sim 1 \mathrm{~mm}$. The cameras recorded probing events in a cuvette (inner dimensions $10.5 \mathrm{~mm} \times 10.5 \mathrm{~mm} \times 6 \mathrm{~mm}$ ) with the probing medium in the center. A micromanipulator allowed translation of the cuvette in three directions to get the ovipositor within the field of view and the depth of field of the cameras. The cuvette was located in a closed-off glass arena (inner dimensions $48 \mathrm{~mm} \times 48 \mathrm{~mm} \times 48 \mathrm{~mm}$ ).

Conversion of camera image pixels to the actual distances was done by using still images of copper specimen support grids for electron microscopy (0050-Cu; Electron Microscopy Sciences). The grid mesh size was measured with a calibrated microscope (Leica M205FA; Leica Microsystems; 20× magnification), after which the grids were put in a cuvette and embedded in the gel, which was also used in the experiments (see below). The difference in vertical position of the cameras was corrected based on the mean vertical coordinate (depth) of the most distal part of the tracked ovipositor tip in both camera views.

An additional camera (piA640-210-gm, 50 fps; Basler AG; with a Nikkor AF-50 lens; Nikon) recorded the spatial orientation of the probing wasp from above. The conversion from pixels to actual lengths for the images of this camera was calculated based on the known dimensions of the cuvette. Image time stamps were used to match the recordings between non-synchronized cameras.

Probing behavior was recorded (Fig. 7A) in substrates with two different densities to assess the effect of substrate properties. The substrate contained commercially available apple juice $(10 \%)$, tap water $(90 \%)$, and gelling agent (Phytagel; SigmaAldrich) in either $0.02 \mathrm{~g} \mathrm{ml}^{-1}(2 \%)$ or $0.04 \mathrm{~g} \mathrm{ml}^{-1}(4 \%)$. The bottom of each cuvette contained a live, 4 - to 7-d-old C. capitata larva and some of its rearing medium, covered with a fine cloth. A piece of wet filter paper with thinly spread larval medium was on the top of the substrate. The presence of these cues induced and prolonged 

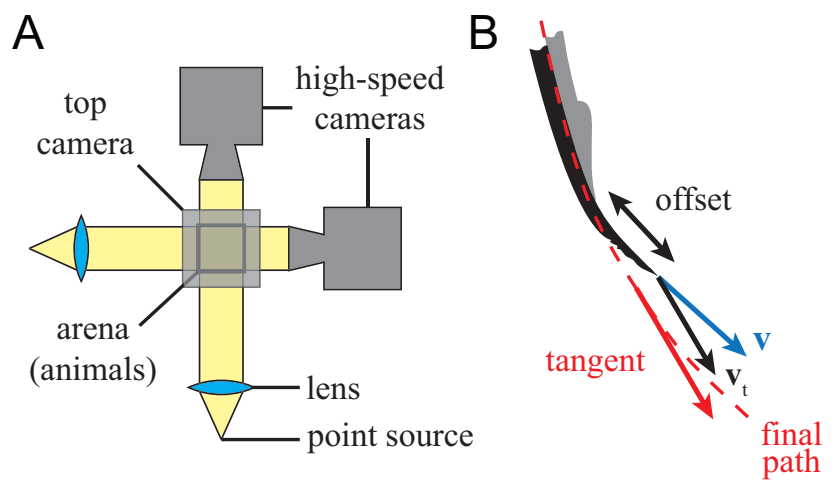

Fig. 7. Experimental setup and calculation of insertion speed. (A) Two high-speed cameras were positioned perpendicular to each other, each aligned with a near-collimated light beam (yellow). The arena with the substrate filled cuvette and the animals was located in the field of view of both cameras. The third camera was located above the experimental arena, directed downward. (B) Schematics of the ovipositor during insertion in 2D (only two valves are shown). The tangential projection $\left(\mathbf{v}_{\mathbf{t}}\right)$ of the valve velocity ( $\mathbf{v})$ along the final centerline (dashed, red line) was taken as the insertion speed. Insertion speed was calculated from the velocity of the foremost valve.

the probing behaviors of the wasps. At the start of an experiment, several wasps were put in the arena, and non-responsive females (i.e., those not motivated to probe) were removed until only one responsive animal was left. Upon initiation of probing, the position of the arena was manually adjusted to have the ovipositor in focus, after which no further adjustments were made to avoid movement artefacts. After a successful recording, wasps were isolated to ensure analysis of probing behavior of each animal in both $2 \%$ and $4 \%$ substrate. Each gel was used for several wasps until either the gel started showing signs of drying or the transparency reduced too much due to the number of probing paths.

\section{Data analysis}

\section{Automatic tracking}

A single animal usually probed the substrate more than once. We defined all movements of the ovipositor made through a single puncture point of the surface as one insertion session. Such a session in general consisted of several insertions, which we defined as movement of the ovipositor into the substrate away from the puncture point. Image sequences of insertion sessions were split into individual insertions and cropped such that they contained only the full insertion trajectory.

In these image sequences, the ovipositors were segmented by using custom-built code in Matlab (Versions R2013a and R2016b; MathWorks). First, the background (average of first five frames of each insertion) was subtracted from all images. This was followed by a conversion to binary images based on a threshold adapted to 
each image sequence such that the ovipositor silhouette was clearly recognizable. Smaller objects and artefacts were removed by a dilation and erosion procedure, sometimes supplemented with manual input. This section of the ovipositor that was visible only in the first five frames had to be segmented from the background images separately and then combined with other images to obtain a complete trajectory. The segmentation of the ovipositor in the background images was done with ilastik (ilastik: Interactive Learning and Segmentation Toolkit; Version 1.1).

By using the cleared images, the centerline of the ovipositor in each frame was obtained by skeletonizing the segmented ovipositor and fitting a cubic smoothed spline curve through it (goodness of fit was determined by visual inspection). Because the tip of the skeletonized ovipositor was often irregular due to irregularities in the gel, the distal end of the skeleton was not used for fitting. The position of the most distal part of the ovipositor tip was determined by linear extrapolation of the centerline up to the boundary of the segmented ovipositor within $>7.5^{\circ}$.

Images from the top camera were cropped and converted to binary images based on thresholds adapted to the individual image sequence so that the silhouette of the animal was clearly recognizable. This was followed by morphological open and close operations to improve the segmentation. Ellipses were then fitted to segmented animals in each frame. The orientation (azimuth) of the animal was defined by the direction angle of the ellipse's major axis directed toward the animal's head with the $Y$ axis of the world coordinate system (defined below).

The 3D probing trajectories were obtained by combining the two perpendicular coordinate systems of the cameras into a right-handed world coordinate system. The $3 \mathrm{D}$ trajectories were smoothed with a quintic spline function to ensure continuous second derivatives needed for calculating the curvature. All 3D trajectories were aligned such that the first insertion was set to the common origin $(0,0,0)$. The curves were then rotated around the $Z$ axis to correct for the orientation of the animals so that the body axis was aligned with the positive $Y$ axis. For each point of the trajectories, the amplitude of the curvature $\kappa$ of the ovipositor was calculated by using the expression obtained from the general formula for a parametrically defined curve $^{77}$ :

$$
\kappa=\frac{\left\|\mathbf{1}(\mathrm{s})^{\prime} \times \mathbf{1}(\mathrm{s})^{\prime \prime}\right\|}{\left\|\mathbf{l}(\mathrm{s})^{\prime}\right\|^{3}}=\sqrt{\frac{\left(y^{\prime} z^{\prime \prime}-y^{\prime \prime} z^{\prime}\right)^{2}+\left(x^{\prime \prime} z^{\prime}-x^{\prime} z^{\prime \prime}\right)^{2}+\left(x^{\prime} y^{\prime \prime}-x^{\prime \prime} y^{\prime}\right)^{2}}{\left(x^{\prime 2}+y^{\prime 2}+z^{\prime 2}\right)^{3}}}
$$

where $\mathbf{l}_{(\mathrm{s})}$ is the vector function of the curve expressed as function of its arc length (s), and ' and "denote the first and second derivatives with respect to the arc length. The dimensionless curvature was obtained by multiplying $\kappa$ with the diameter of the ovipositor ( $30 \mu \mathrm{m}$, estimated from SEM images). 
Probing velocity was calculated by taking the gradient of the filtered position data of the most distal part of the ovipositor tip ( $2^{\text {nd }}$ order low-pass Butterworth filter, cutoff frequency $15 \mathrm{~Hz}$ applied twice using Matlab 'filtfilt' function, effectively using a $4^{\text {th }}$ order filter).

In the analysis of the 3D dataset, the insertion speed was taken as the magnitude of the component of the velocity vector tangential to the centerline of the final path at each time point (Fig. 7B). Retractions were not analyzed. The speed of the foremost valve was taken as the insertion speed of the entire ovipositor.

Analysis of valve kinematics in 2D was done for three insertion examples where individual valves were clearly in focus (see below). Valve insertion and retraction speeds were calculated as the magnitude of the components of the instantaneous velocity tangential to the instantaneous ovipositor centerline in each frame. We also discriminated between the speed of the dorsal and the foremost ventral valve.

\section{Analysis of valve kinematics}

The high magnification used, resulted in a narrow depth of field (DoF), which limited the tracking to a single camera view and to only parts of insertions. We chose image sequences where the ovipositor was moving within the plane of the DoF (i.e., did not curve in the view of the second camera). In our backlit images, only silhouettes of the valves were discernible, so only the most protruded (foremost) ventral valve could be recognized. The most distal ends of the dorsal and the foremost ventral valve tips were manually digitized in all frames where the ovipositor tip was in focus. The dorsal valve could easily be distinguished from the ventral valves based on its morphology (Fig. 1A).

The digitized points together with the frame-by-frame centerlines were used to calculate the tangential velocity of the valves as described above. In addition, the distance between the digitized most distal ends of the valve tips in each frame was taken as the offset between the valves. Positive offset denotes protraction of the dorsal valve, and negative denotes the protraction of the foremost ventral valve.

The ovipositor was segmented as described above, except for the example shown in Fig. 6C, which was done by using ilastik. In the latter example, the segmentation routine in Matlab yielded unsatisfactory results due to a complex background of the images. Automated tracking of the insertion was accurate, so there was no need to remove spurious ends of the skeletons and the extrapolation of the centerlines (as above).

Orientation of the ovipositor tip during insertion was calculated from the end part of the centerline. The length of the end part was taken as one-third of the length of 
the dorsal valve tip. We calculated the angle between the orientation of the ovipositor tip and the horizontal plane. We filtered the orientation data in time $\left(2^{\text {nd }}\right.$ order low-pass Butterworth filter, cutoff frequency $18.75 \mathrm{~Hz}$ applied twice using the Matlab 'filtfilt' function), which reduced the frame to frame artefacts, but retained the overall temporal characteristics (Fig. S7A-C). We then transformed the orientation data from the image frame of reference into the ovipositor frame of reference by subtracting the baseline orientation $\left(2^{\text {nd }}\right.$ order low-pass Butterworth filter, cutoff frequency $1 \mathrm{~Hz}$ applied twice by using the Matlab 'filtfilt' function) from the direction data (Fig. S7D-F).

The data are available on Dryad (http://dx.doi.org/10.5061/dryad.8bc95).

\section{Estimating the accelerations and the net forces on the valves}

The net forces on the valves (Figs S3-S5) were estimated by taking the second derivative of the filtered position data, combined with a simplified cylindrical model of the ovipositor assuming simple material properties (Fig. S8). The density of the cuticle was taken from ref. [78]. For details of force calculations see SI Materials and methods.

\section{Acknowledgements}

We thank Francisco Beitia (Instituto Valenciano de Investigaciones Agrarias) for providing parasitoids and Carlos Caćeres (International Atomic Energy Agency) for providing fruit-flies for our colony; Marleen Kamperman and Marco Dompe'Physical Chemistry and Soft Matter, Wageningen University and Research) for their assistance with the rheological measurements of the substrates; Henk Schipper, Remco Pieters, and Karen Leon-Kloosterziel for technical support and animal care; Cees Voesenek for advice on analytical methods; Dimitra Dodou (Delft University of Technology) for advice on the experiments; and the user committee of the Netherlands Organization for Scientific Research Division Applied and Engineering Sciences (NWO TTW) WASP project and the members of the research group for their useful discussions. This work was supported by NWO domain applied and engineering sciences (NWO TTW). 


\section{References}

[1] Le LANNic J \& NÉnOn JP (1999). Functional morphology of the ovipositor in Megarhyssa atrata (Hymenoptera, Ichneumonidae) and its penetration into wood. Zoomorphology 119, 73-79.

[2] Ovruski S, Aluja M, Sivinski J, \& Wharton R (2000). Hymenopteran parasitoids on fruit-infesting Tephritidae (Diptera) in Latin America and the Southern United States: Diversity, distribution, taxonomic status and their use in fruit fly biological control. Integrated Pest Management Reviews 5, 81-107.

[3] Belshaw R, Grafen A, \& Quicke DLJ (2003). Inferring life history from ovipositor morphology in parasitoid wasps using phylogenetic regression and discriminant analysis. Zoological Journal of the Linnean Society 139, 213-228.

[4] SмITH EL (1970). Evolutionary morphology of the external insect genitalia. 2. Hymenoptera. Annals of the Entomological Society of America 63, 1-27.

[5] Quicke DLJ, Fitton MG, Tunstead JR, Ingram SN, \& Gaitens PV (1994). Ovipositor structure and relationships within the Hymenoptera, with special reference to the Ichneumonoidea. Journal of Natural History 28, 635-682.

[6] Ghara M, Kundanati L, \& Borges RM (2011). Nature's Swiss Army knives: ovipositor structure mirrors ecology in a multitrophic fig wasp community. PloS one 6, e23642.

[7] SCHUdDER GGE (1961). The comparative morphology of the insect ovipositor. Transactions of the Royal Entomological Society of London 113, 2-40.

[8] Lyngnes R (1960). Shape and function of the ovipositor in the three Hymenopterous species: Ephialtes extensor Thom. (Ichneumonidae), Spathius exarator L. (Braconidae), and Plutothrix coelius Walk. (Chalcididae). Norsk Entomologisk Tidsskrift 11, 122-134.

[9] KING PE (1962). The muscular structure of the ovipositor and its mode of function in Nasonia vitripennis (Walker) (Hymenoptera: Pteromalidae). Proceedings of Royal Entomological Society London. Series A, General Entomology 37, 121-128.

[10] Quicke DLJ (2015). The braconid and ichenumonid parasitoid wasps: biology, systematics, evolution and ecology. John Wiley \& Sons, Ltd, first edn.

[11] Vilhelmsen L \& TuRrisi GF (2011). Per arborem ad astra: morphological adaptations to exploiting the woody habitat in the early evolution of Hymenoptera. Arthropod Structure \& Development 40, $2-20$.

[12] VilheLmSEn L (2000). Before the wasp-waist: comparative anatomy and phylogenetic implications of the skeleto-musculature of the thoraco-abdominal boundary region in basal Hymenoptera (Insecta). Zoomorphology 119, 185-221.

[13] Sharkey MJ (2007). Phylogeny and classification of Hymenoptera. Zootaxa 1668, e548.

[14] Davis RB, Baldauf SL, \& Mayhew PJ (2010). The origins of species richness in the Hymenoptera: insights from a family-level supertree. BMC evolutionary biology 10, 109.

[15] Peters RS, Krogmann L, Mayer C, Donath A, Gunkel S, Meusemann K, Kozlov A, Podsiadlowski L, Petersen M, lanfear R, Diez PA, Heraty J, KJer KM, Klopfstein S, Meier R, Polidori C, Thomas S, Shanlin L, Zhou X, Wappler T, Rust J, Misof B, \& Niehus O (2017). Evolutionary history of the Hymenoptera. Current Biology 27, 1013-1018.

[16] Matushkina N \& Gorb S (2007). Mechanical properties of the endophytic ovipositor in damselflies (Zygoptera, Odonata) and their oviposition substrates. Zoology 110, 167-175.

[17] Polidori C, García AJ, \& Nieves-Aldrey JL (2013). Breaking up the wall: metal-enrichment in ovipositors, but not in mandibles, co-varies with substrate hardness in gall-wasps and their associates. PloS one 8, e70529.

[18] Kundanati L \& Gundiah N (2014). Biomechanics of substrate boring by fig wasps. Journal of Experimental Biology 217, 1946-1954. 
[19] Sмiтн EL (1972). Biosystematics and morphology of Symphyta - III external genitalia of Euura (Hymenoptera: Tenthredinidae): sclerites, sensilla, musculature, development and oviposition behaviour. International Journal of Insect Morphology and Embryology 1, 321-65.

[20] FIeld SA \& Austin AD (1994). Anatomy and mechanics of the telescopic ovipositor system of Scelio latreille (Hymenoptera: Scelionidae) and related genera. International Journal of Insect Morphology and Embryology 23, 135-158.

[21] Vilhelmsen L, Isidoro N, Romani R, Basibuyuk HH, \& Quicke DLJ (2001). Host location and oviposition in a basal group of parasitic wasps: the subgenual organ, ovipositor apparatus and associated structures in the Orussidae (Hymenoptera, Insecta). Zoomorphology 121, 63-84.

[22] Heatwole H, Davis DM, \& Wenner AM (1962). The behaviour of Megarhyssa, a genus of parasitic hymenopterans (Ichneumonidae: Ephialtinae). Zeitschrift fur Tierpsychologie - Journal of Comparative Ethology 19, 652-664.

[23] VINCENT JFV \& KING MJ (1995). The mechanism of drilling by wood wasp ovipositors. Biomimetics 3, 187-201.

[24] Vilhelmsen L (2003). Flexible ovipositor sheaths in parasitoid Hymenoptera (Insecta). Arthropod Structure \& Development 32, 277-287.

[25] Quicke DLJ, Fitton M, \& Harris J (1995). Ovipositor steering mechanisms in braconid wasps. Journal of Hymenoptera Research 4, 110-120.

[26] Quicke DLJ (1991). Ovipositor mechanics of the braconine wasp genus Zaglyptogastra and the ichneumonid genus Pristomerus. Journal of Natural History 25, 971-977.

[27] Pollard DG (1969). Directional control of the stylets in phytophagous Hemiptera. Proceedings of the Royal Entomological Society of London. Series A, General Entomology 44, 173-185.

[28] Elias LG, Teixeira SP, Kjellberg F, \& Santinelo Pereira RA (2012). Diversification in the use of resources by Idarnes species: bypassing functional constraints in the fig-fig wasp interaction. Biological Journal of the Linnean Society 106, 114-122.

[29] Abolhassani N, Patel R, \& Moallem M (2007). Needle insertion into soft tissue: A survey. Medical engineering \& physics 29, 413-431.

[30] Misra S, Reed KB, Douglas AS, Ramesh KT, \& Okamura AM (2008). Needle-tissue interaction forces for bevel-tip steerable needles. Proceedings of the 2nd Biennial IEEE/RAS-EMBS International Conference on Biomedical Robotics and Biomechatronics 224-231.

[31] Abayazid M, Roesthuis RJ, Reilink R, \& Misra S (2013). Integrating deflection models and image feedback for real-time flexible needle steering. IEEE Transactions on Robotics 29, 542-553.

[32] Le Ralec A, Rabasse JM, \& Wajnberg E (1996). Comparative morphology of the ovipositor of some parasitic Hymenoptera in relation to characteristics of their hosts. The Canadian Entomologist 128, 413-433.

[33] Gerling D, Quicke DLJ, \& Orion T (1998). Oviposition mechanisms in the whitefly parasitoids Encarsia transvena and Eretmocerus mundus. BioControl 43, 289-297.

[34] Ko SY, Frasson L, \& Rodriguez y Baena F (2011). Closed-loop planar motion control of a steerable probe with a 'programmable bevel' inspired by nature. IEEE Transactions on Robotics 27, 970-983.

[35] Quicke DLJ \& Fitton MG (1995). Ovipositor steering mechanisms in parasitic wasps of the families Gasteruptiidae and Aulacidae (Hymenoptera). Proceedings of The Royal Society: Biological Sciences 261, 98-103.

[36] PollaRd DG (1971). The use of polyporus for the investigation of stylet behaviour in the Hemiptera. Entomologia Experimentalis et Applicata 14, 283-296.

[37] Compton S \& Nefdt R (1988). Extra-long ovipositors in chalcid wasps: some examples and observations. Antenna 12, 102-105.

[38] Sears P \& Dupont P (2006). A steerable needle technology using curved concentric tubes. 2006 IEEE/RSJ International Conference on Intelligent Robots and Systems 2850-2856. 
[39] Parittotokkaporn T, Frasson L, Schneider A, Huq S, Davies Bl, Degenaar P, Biesenack J, \& Rodriguez y Baena FM (2009). Soft tissue traversal with zero net force: Feasibility study of a biologically inspired design based on reciprocal motion. Proceedings of the 2008 IEEE International Conference on Robotics and Biomimetics 80-85.

[40] Frasson L, Ko SY, Turner A, Parittotokkaporn T, Vincent JF, \& Rodriguez y Baena F (2010). STING: a soft-tissue intervention and neurosurgical guide to access deep brain lesions through curved trajectories. Proceedings of the Institution of Mechanical Engineers, Part H: Journal of Engineering in Medicine 224, 775-788.

[41] Roesthuis RJ, Van Veen YR, Jahya A, \& Misra S (2011). Mechanics of needle-tissue interaction. 2011 IEEE/RSJ International Conference on Intelligent Robots and Systems 2557-2563.

[42] Ko SY \& Rodriguez y Baena F (2012). Trajectory following for a flexible probe with state/input constraints: An approach based on model predictive control. Robotics and Autonomous Systems 60, 509-521.

[43] Elgezua I, Kobayashi Y, \& Fujie MG (2013). Survey on current state-of-the-art in needle insertion robots: open challenges for application in real surgery. Procedia CIRP 5, 94-99.

[44] Consoli FL, Kitajima EW, \& Postali Parra JR (1999). Sensilla on the antenna and ovipositor of the parasitic wasps Trichogramma galloi Zucchi and T. pretiosum Riley (Hym., Trichogrammatidae). Microscopy Research and Technique 45, 313-324.

[45] Hawke SD, Farley RD, \& Greany PD (1973). The fine structure of sense organs in the ovipositor of the parasitic wasp, Orgilus lepidus Muesebeck. Tissue and Cell 5, 171-184.

[46] Van Lenteren JC, Ruschioni S, Romani R, van Loon JJA, Qiu YT, Smid HM, Isidoro N, \& Bin F (2007). Structure and electrophysiological responses of gustatory organs on the ovipositor of the parasitoid Leptopilina heterotoma. Arthropod Structure \& Development 36, 271-276.

[47] Sнан ZA (2012). Morphology, ultrastructure, and probable functions of the sense organs on the ovipositor stylets of the hymenoptran parasitoid, Venturia canescens (Gravenhorst). Microscopy research and technique $75,876-883$.

[48] VAN LeNTERen JC, Isidoro N, \& BIN F (1998). Functional anatomy of the ovipositor clip in the parasitoid Leptopilina heterotoma (Thompson) (Hymenoptera: Eucolidae), a structure to grip escaping host larvae. International Journal of Insect Morphology and Embryology 27, 263-268.

[49] VINSON SB (1976). Host selection by insect parasitoids. Annual Review of Entomology 21, 109-133.

[50] SPRAdbery JP (1970). Host finding by Rhyssa persuasoria (L.) in ichneumonid parasite of siricid woodwasps. Animal Behaviour 18, 103-114.

[51] Ghara M, Ranganathan Y, Krishnan A, Gowda V, \& Borges RM (2014). Divvying up an incubator: How parasitic and mutualistic fig wasps use space within their nursery microcosm. ArthropodPlant Interactions 8, 191-203.

[52] Кім BH, Кім HK, \& LeE SJ (2011). Experimental analysis of the blood-sucking mechanism of female mosquitoes. The journal of experimental biology 214, 1163-1169.

[53] Gordon RM \& Lumsden WHR (1939). A study of the behaviour of the mouth-parts of mosquitoes when taking up blood from living tissue together with some observations on the ingestion of microfiliarie. Annals of tropical medicine and parasitology 33, 259-278.

[54] Griffiths RB \& GoRdon RM (1952). An apparatus which enables the process of feeding by mosquitoes to be observed in the tissues of a live rodent; together with an account of the ejection of saliva and its significance in malaria. Annals of tropical medicine and parasitology 46, 311-319.

[55] Kong X \& WU C (2009). Measurement and prediction of insertion force for the mosquito fascicle penetrating into human skin. Journal of Bionic Engineering 6, 143-152.

[56] Choumet V, Attout T, Chartier L, Khun H, Sautereau J, Robbe-Vincent A, Brey P, Huerre M, \& BAIN O (2012). Visualizing non infectious and infectious Anopheles gambiae blood feedings in naive and saliva-immunized mice. PloS one 7 , e50464.

[57] Kong XQ \& WU CW (2010). Mosquito proboscis: An elegant biomicroelectromechanical system. Physical review E - statistical, nonlinear, and soft matter physics 82, 011910. 
[58] Izumi H, Yajima T, Aoyagi S, Tagawa N, Arai Y, Hirata M, \& Yorifuji S (2008). Combined harpoonlike jagged microneedles imitating mosquito's proboscis and its insertion experiment with vibration. IEEJ transactions on electrical and electronic engineering 3, 425-431.

[59] Freeman TP, Buckner JS, Nelson DR, Chu CC, \& Henneberry TJ (2001). Stylet penetration by Bemisia argentifolii (Homoptera: Aleyrodidae) into host leaf tissue. Annals of the Entomological Society of America 94, 761-768.

[60] Leopold RA, Freeman TP, Buckner JS, \& Nelson DR (2003). Mouthpart morphology and stylet penetration of host plants by the glassy-winged sharpshooter, Homalodisca coagulata, (Homoptera: Cicadellidae). Arthropod Structure \& Development 32, 189-199.

[61] Frasson L, Ferroni F, Ko SY, Dogangil G, \& Rodriguez y Baena F (2011). Experimental evaluation of a novel steerable probe with a programmable bevel tip inspired by nature. Journal of Robotic Surgery 6, 189-197.

[62] Webster RJ, Kim JS, Cowan NJ, Chirikjian GS, \& Okamura AM (2006). Nonholonomic modeling of needle steering. The International Journal of Robotics Research 25, 509-525.

[63] Dai W, Pan L, Lu Y, Jin L, \& Zhang C (2014). External morphology of the mouthparts of the whitebacked planthopper Sogatella furcifera (Hemiptera: Delphacidae), with special reference to the sensilla. Micron 56, 8-16.

[64] Zhao L, Dai W, Zhang C, \& Zhang Y (2010). Morphological characterization of the mouthparts of the vector leafhopper Psammotettix striatus (L.) (Hemiptera: Cicadellidae). Micron 41, 754-759.

[65] Kang KS, Veeder GT, Mirrasoul PJ, Kaneko T, \& Cottrell IW (1982). Agar-like polysaccharide produced by a Pseudomonas species: Production and basic properties. Applied and Environmental Microbiology 43, 1086-1091.

[66] Nussinovitch A, AK MM, Normand MD, \& Peleg M (1990). Characterization of gellan gels by uniaxial compression, stress relaxation and creep. Journal of Texture Studies 21, 37-49.

[67] FINNey EEJ (1967). Dynamic elastic properties of some fruits during growth and development. Journal of Agricultural Engineering Research 12, 249-256.

[68] Leyva JL, Browning HW, \& Gilstrap FE (1991). Effect of host fruit species, size, and color on parasitization of Anastrepha ludens (Diptera: Tephritidae) by Diachasmimorpha longicaudata (Hymenoptera: Braconidae). Environmental Entomology 20, 1469-1474.

[69] Segura DF, Nussenbaum AL, \& Viscarret MM (2016). Innate host habitat preference in the parasitoid Diachasmimorpha longicaudata: functional significance and modifications through learning. PloS One 11, 0152222.

[70] Fridley RB, Bradley RA, Rumsey JW, \& Adrian PA (1968). Some aspects of elastic behavior of selected fruits. Transactions of the American Society of Agricultural Engineers 11, 46-49.

[71] Delwiche MJ, Mcdonald T, \& Bowers SV (1987). Determination of peach firmness by analysis of impact forces. Transactions of the American Society of Agricultural Engineers 30, 249-254.

[72] Fidelibus MW, Teixeira AA, \& Davies FS (2002). Mechanical properties of orange peel and fruit treated pre-harvest with gibberellic acid. Transactions of the American Society of Agricultural Engineers 45, 1057-1062.

[73] SINGH KK \& Reddy BS (2006). Post-harvest physico-mechanical properties of orange peel and fruit. Journal of Food Engineering 73, 112-120.

[74] Misra S, Reed KB, Schafer BW, Ramesh KT, \& Okamura AM (2010). Mechanics of flexible needles robotically steered through soft tissue. International Journal of Robotic Research 29, 1640-1660.

[75] Ko SY, Davies BL, \& Rodriguez y Baena F (2010). Two-dimensional needle steering with a "programmable bevel" inspired by nature: Modeling preliminaries. Intelligent Robots and Systems (IROS), 2010 IEEE/RSJ International Conference on 2319-2324.

[76] Jansson PE, Bengt L, Elaborated P, \& Pseudomonas BY (1983). Structural studies of gellan gum, an extracellular polysaccharide elaborated by Pseudomonas elodea. Carbohydrate Research 124, 135139. 
[77] O'Neal B (1966). Elementary differential geometry. London: Academic Press, Inc. (London) ltd., first edn.

[78] Vincent JFV \& Wegst UGK (2004). Design and mechanical properties of insect cuticle. Arthropod Structure \& Development 33, 187-199. 


\section{Supporting information}

\section{SI Materials and methods}

\section{SI Rearing and propagation}

\section{Environment}

The insects were kept in $(30 \mathrm{~cm} \times 30 \mathrm{~cm} \times 30 \mathrm{~cm}$ cages (Bug-Dorm; MegaView Science) in incubators (Panasonic) at $24^{\circ} \mathrm{C}$, relative humidity of $40-50 \%$, and 12-h light/dark cycle. Each cage contained an Erlenmeyer flask filled with tap water and a rolled-up filter paper sticking out. Wasps were fed with commercially available honey spread on the side of the cages and flies with a mixture of glucose and brewer's yeast dissolved in tap water (for details, see below). Food and water was checked regularly and refilled when needed.

\section{Flies}

Fly eggs were collected with an 'artificial fruit' - a plastic bottle with small holes through which the flies lay their eggs. Inside the bottle was a wet filter paper to keep the eggs from drying. A sufficient number of eggs could be harvested form a single cage when the bottle was kept in the cage for at least half a day/night. The eggs were washed out with tap water and aerated for 8-24 h before they were transferred onto larval medium (for details, see below) with a plastic Pasteur pipette. The Petri dish was covered with its lid to prevent drying of the medium-larvae should be kept at a higher relative humidity: 60-70\%. After approximately a week, the larvae went through all their larval stages and began to pupate. The Petri dish was opened and placed in a bigger container with $\sim 50 \mathrm{~g}$ of vermiculite (Pull Rhenen B.V.), humidified with $\sim 50 \mathrm{ml}$ of tap water) for the larvae to crawl into. Adult flies start emerging $\sim 10 \mathrm{~d}$ after pupation. Adults started laying eggs abundantly after a week and a half after emergence.

\section{Wasps}

Wasps that were at least 2 wk old were presented with in 3- to 6-d-old fly larvae. The fly larvae were in a Petri dish filled with medium low enough to allow the wasps to reach to the bottom of the dish. The larvae were kept in the wasp cage for 1 to $2 \mathrm{~d}$. After this time, the Petri dish was taken out, and larvae were left to pupate in vermiculite. Approximately $10 \mathrm{~d}$ after pupation, adult flies started to emerge. After 
the emergence of the flies, the container with the remaining pupae was transferred to a new cage. Wasps started to emerge $\sim 14 \mathrm{~d}$ after pupation.

\section{Fly feed and larval medium}

Fly food consisted of D-(+)-glucose (anhydrous) (Merck), 18 g; brewer's yeast (Acros Organics), $6 \mathrm{~g}$; and tap water, $12 \mathrm{ml}$. The ingredients were mixed and poured into Petri dishes (diameter of $9 \mathrm{~cm}$ ). Larval medium consisted of carrot powder (Jacob Hooy \& Co BV), 60 g; brewer's yeast (Acros Organics), 28 g; methylparaben (Amresco LLC), $0.8 \mathrm{~g}$; sodium benzoate (Alpha Aesar), $1 \mathrm{~g} ; 1 \mathrm{M} \mathrm{HCl}$ (Merck), $40 \mathrm{ml}$; and tap water, $160 \mathrm{ml}$. Wet and dry components were mixed separately and then combined, before pouring into Petri dishes (diameter of $9 \mathrm{~cm}$ ). The mentioned amount is sufficient for approximately seven Petri dishes. Excess of the medium was stored in plastic bags in a refrigerator.

\section{SI Rheological measurements}

The storage $\left(G^{\prime}\right)$ and loss $\left(G^{\prime \prime}\right)$ moduli (Fig. S1) were obtained as described in the main text. The values were averaged across frequencies and used to determine the magnitude of the dynamic shear modulus $\left(\left|G^{*}\right|\right)$ of the gels by using the formula: $\left|G^{*}\right|=\sqrt{\left(G^{\prime}\right)^{2}+\left(G^{\prime \prime}\right)^{2}}$. The dynamic shear modulus was used to calculate the dynamic modulus of elasticity, assuming isotropic material properties and Poisson's ratio $(v)$ of 0.5 by using: $E^{*}=2\left|G^{*}\right|(1+v)$.

\section{SI Estimation of the net valve forces}

Net forces on the valves were approximated by using Newton's second law of motion. Accelerations were obtained by taking the second derivative of the filtered position data. The calculations were performed twice using second order low-pass Butterworth filters-once with a cutoff frequency of $15 \mathrm{~Hz}$ and once with the cutoff frequency of $7.5 \mathrm{~Hz}$. Each time, the filter was applied twice to the position data by using the Matlab filtfilt function, making the filters effectively of the fourth order. Filters were applied twice to retain the overall temporal characteristics of the data. The $15 \mathrm{~Hz}$ cutoff frequency is the same as in the analysis of the valve kinematics, but leads to noisy acceleration data. To reduce the noise, we performed the same calculations using a stronger filter (cutoff frequency of $7.5 \mathrm{~Hz}$ ) (compare left and right columns in Figs S3-S5). The mass of the valves was estimated by using a simple model of the ovipositor. We made the following assumptions. The valves were part of a cylinder (Fig. S8) with a radius of $15 \mu \mathrm{m}$ that was cut longitudinally in three parts: one half and two quarters corresponding to the dorsal and the ven- 
tral valves, respectively. Furthermore, each valve contained cylindrical lumina, of which sizes were estimated from the ovipositor CT scans. The dorsal valve contained two lumina, each with a radius of $2.5 \mu \mathrm{m}$, while each ventral valve contained a lumen with a radius of $4.4 \mu \mathrm{m}$. The lumina were considered to be filled with water $\left(D_{w}=1000 \mathrm{~kg} \mathrm{~m}^{-3}\right)$. The density of the cuticle $\left(D_{c}\right)$ was taken as $1300 \mathrm{~kg} \mathrm{~m}^{-3}$. The length $(L)$ of the valves and the lumina was $5 \mathrm{~mm}$.

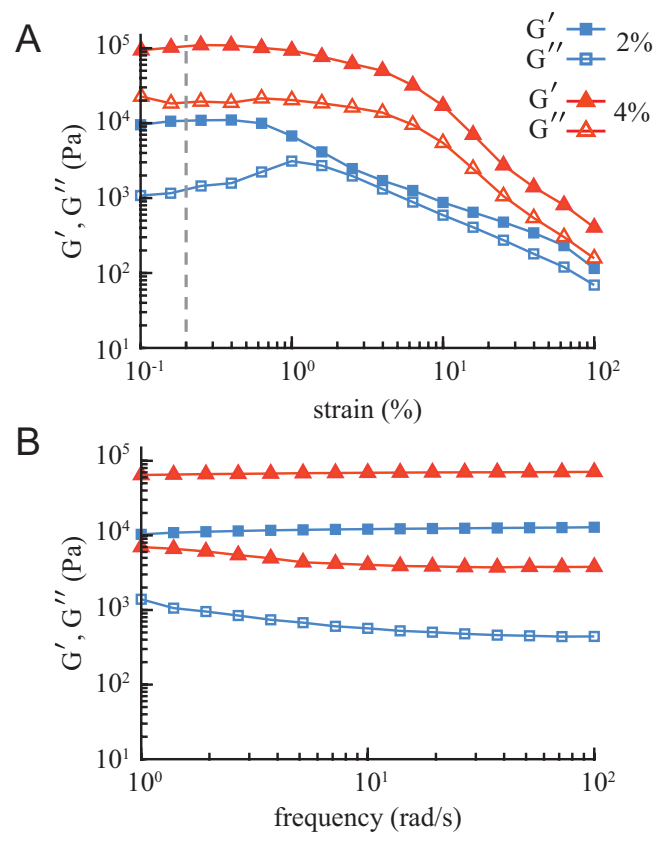

Fig. S1. Gel density correlates with its stiffness. Storage $\left(\mathrm{G}^{\prime}\right)$ and loss $\left(\mathrm{G}^{\prime \prime}\right)$ moduli of the $2 \%$ and $4 \%$ gels are shown in blue and red, respectively. (A) A strain sweep at a constant frequency $(1 \mathrm{~Hz})$ was used to determine the substrate linear strain region. The strain used in frequency sweeps is denoted with a dashed gray line. (B) Frequency sweep performed at a constant strain $(0.2 \%)$. $\mathrm{G}^{\prime}$ is independent of the frequency, whereas a slight decrease in $G^{\prime \prime}$ is seen with increasing frequency.
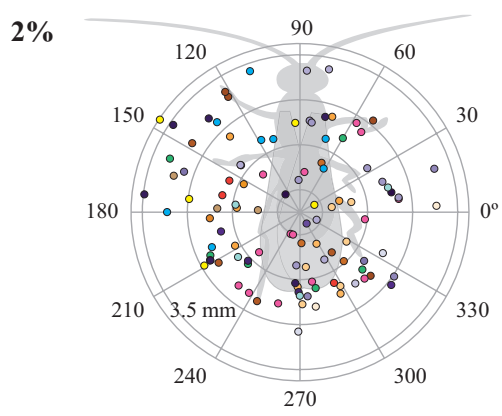

$4 \%$

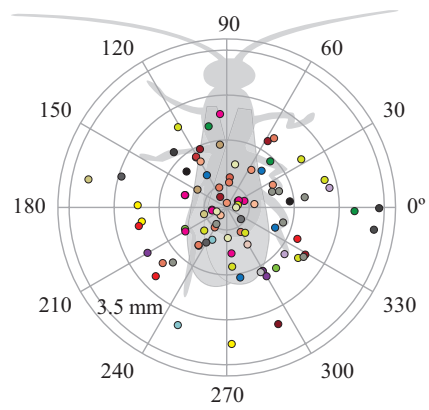

Fig. S2. No directional preference was observed within insertion sessions. Colors represent insertion trajectory endpoints belonging to the same insertion session and corrected for the body orientation of the animal. Directionality was assessed visually, due to the low number of insertions per session. 


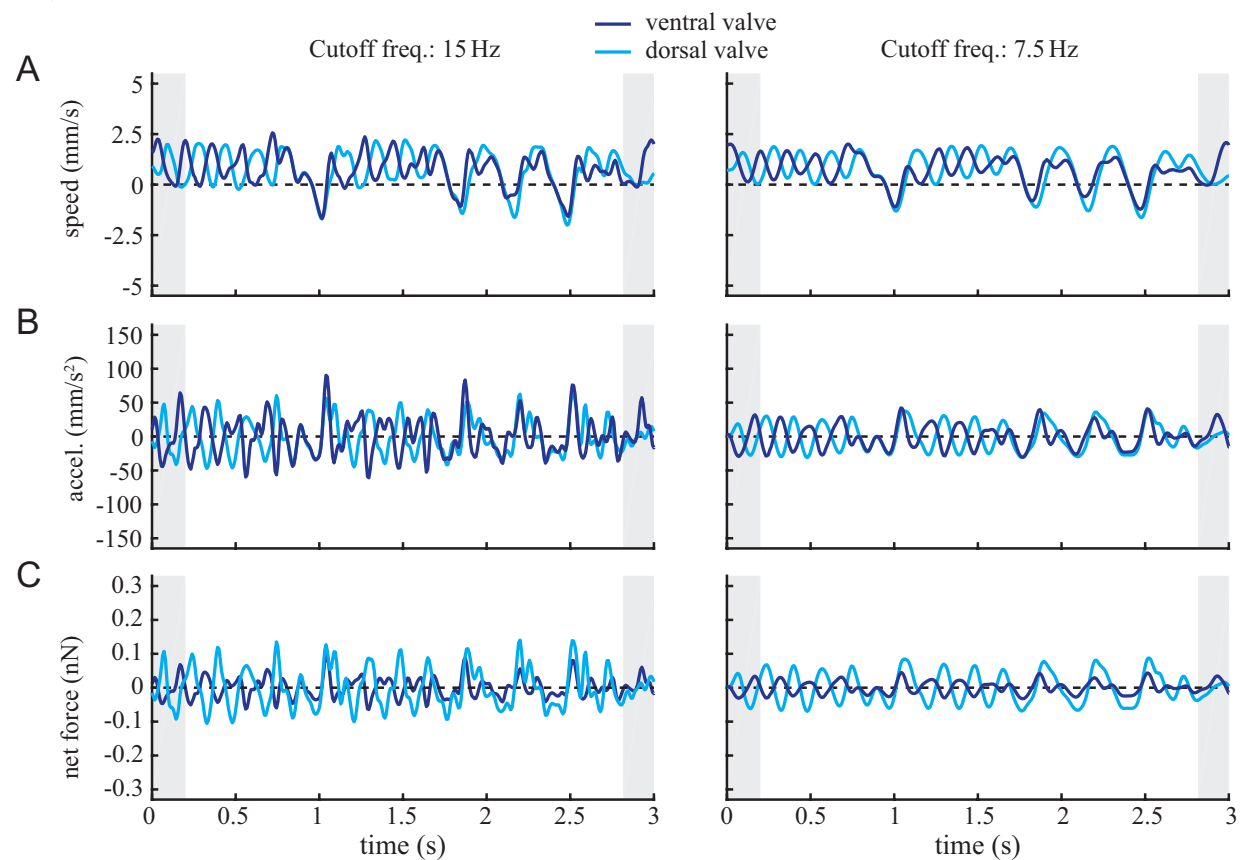

Fig. S3. Valve speed (A), acceleration (B), and the net force (C) during straight insertion. The position data used for calculations were filtered with the effective fourth-order low-pass Butterworth filters with the cutoff frequency of $15 \mathrm{~Hz}$ (Left) and $7.5 \mathrm{~Hz}$ (Right). Stronger filtering was used to reduce the noise in the acceleration data. Dark blue, the foremost ventral valve; light blue, dorsal valve.

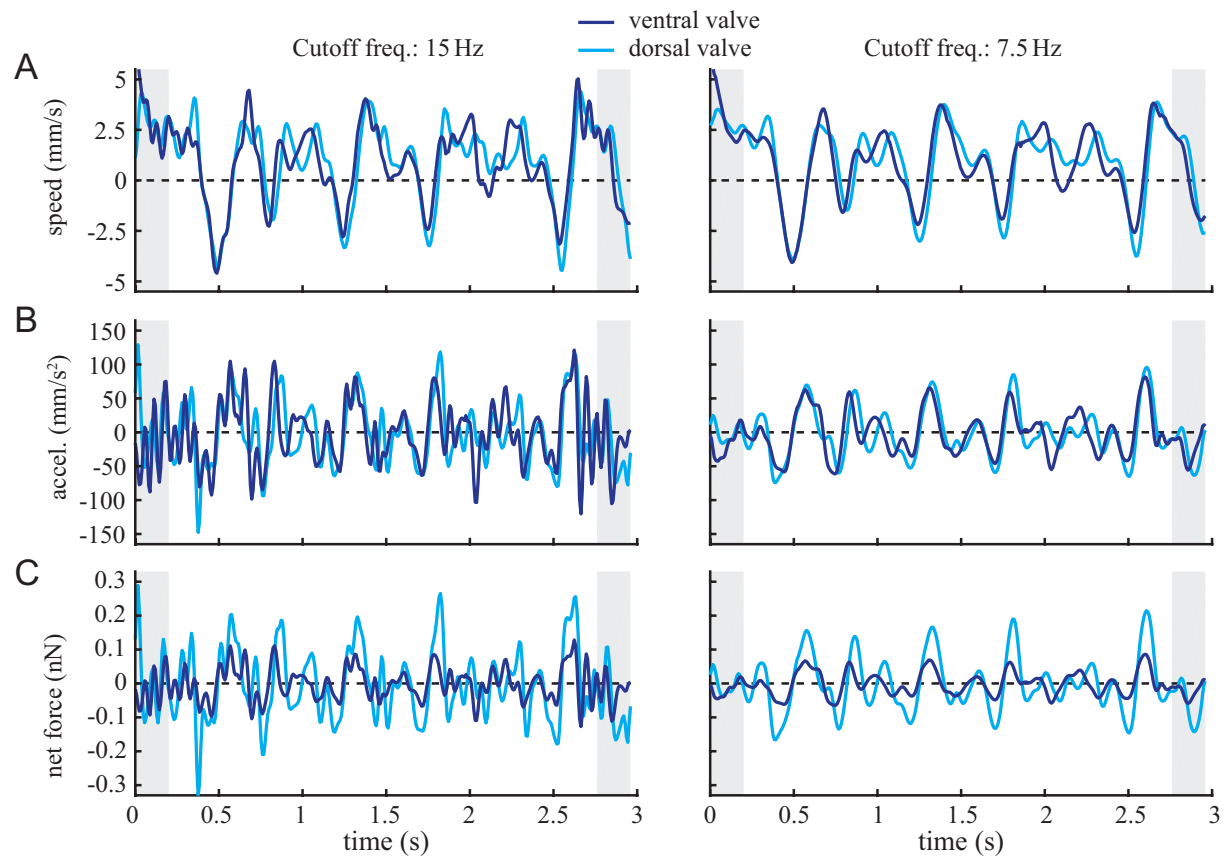

Fig. S4. Valve speed (A), acceleration (B), and the net force (C) during a curved insertion with the 'pushing' method. Graphs were obtained in the same way as in Fig. S3. 

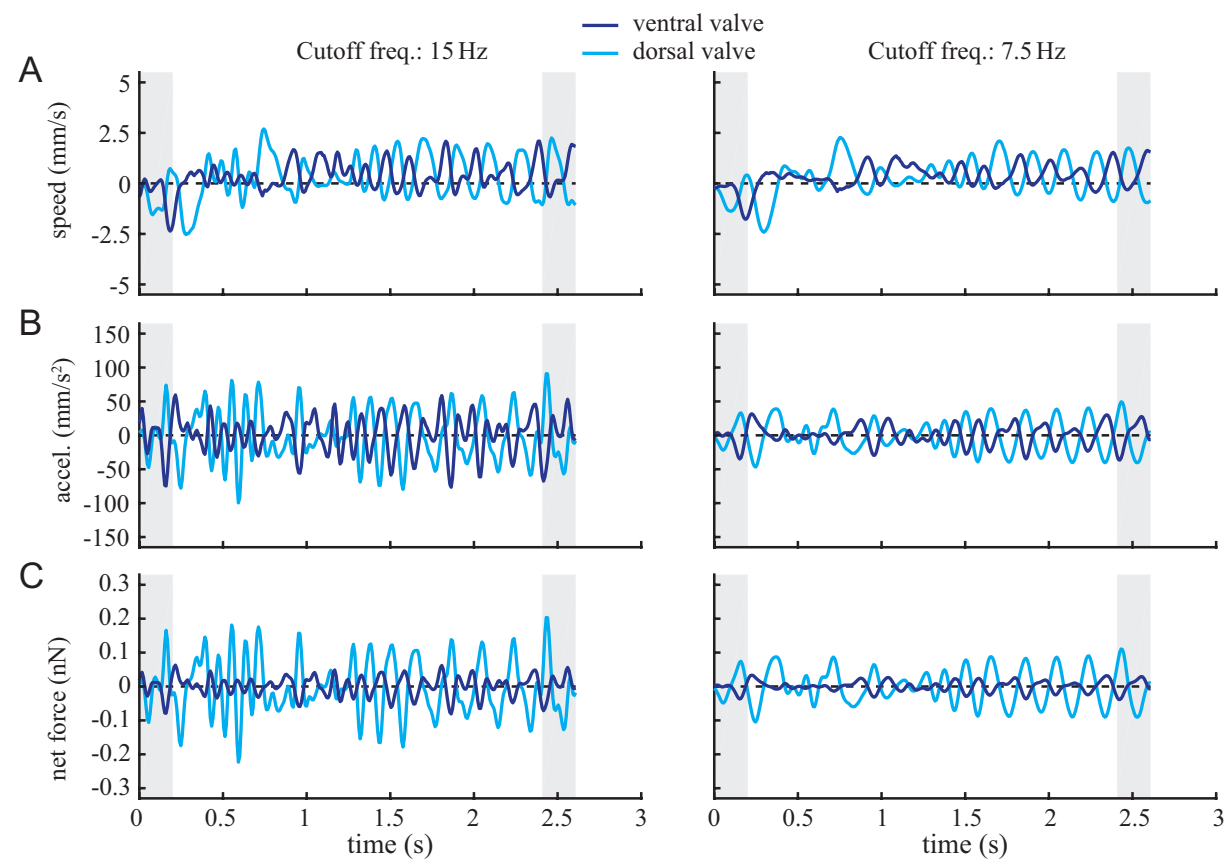

Fig. S5. Valve speed (A), acceleration (B), and the net force (C) during a curved insertion with the 'alternating' method. Graphs were obtained in the same way as in Fig. S3.

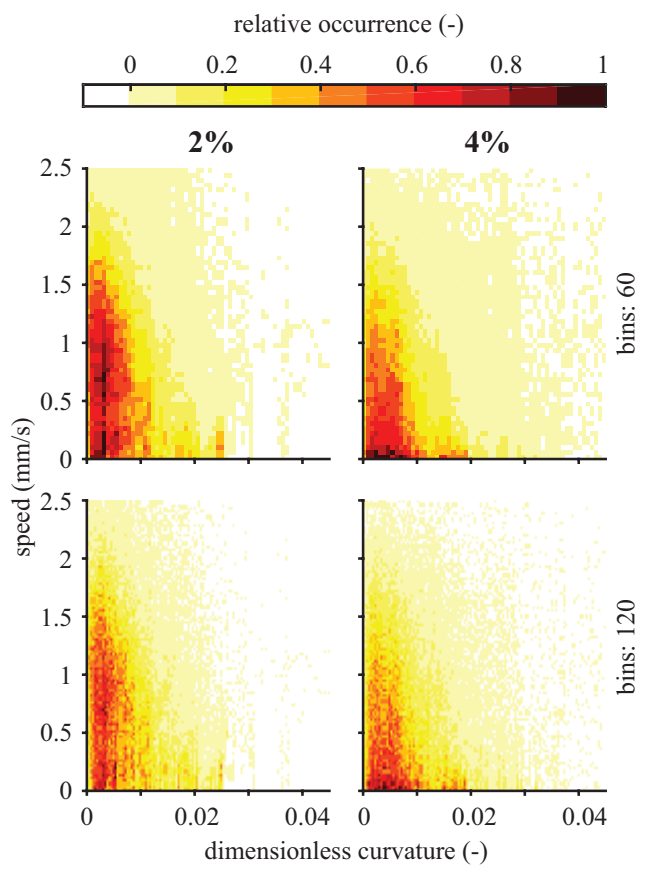

Fig. S6. Bin size has only a small effect on the relative frequency plots of speed and curvature. Upper and Lower show results of axis divisions in 60 and 120 equally sized bins, respectively. General characteristics such as maximal speed, its decline with curvature, and higher curvature in denser substrate are retained irrespective of the bin size and number. 

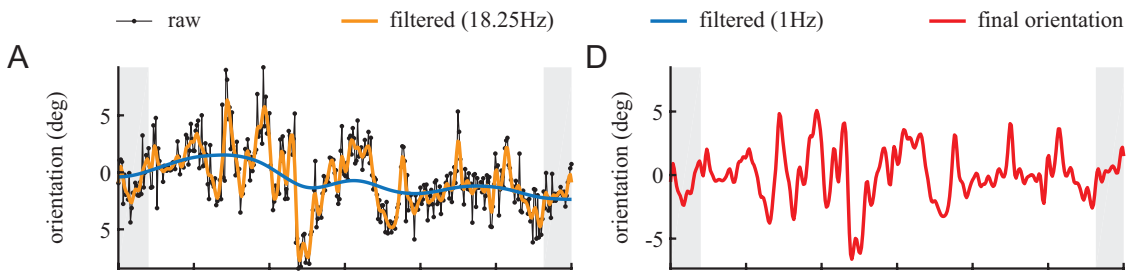

B

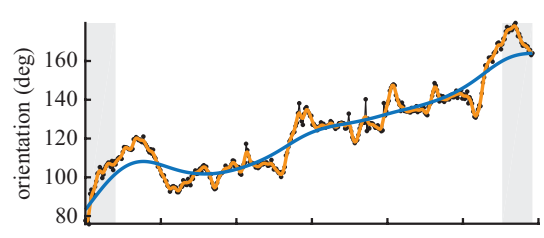

C

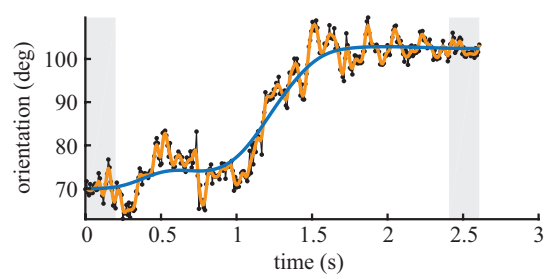

$E$

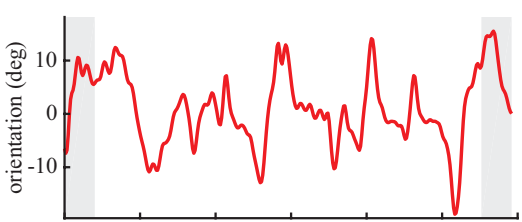

$\mathrm{F}$

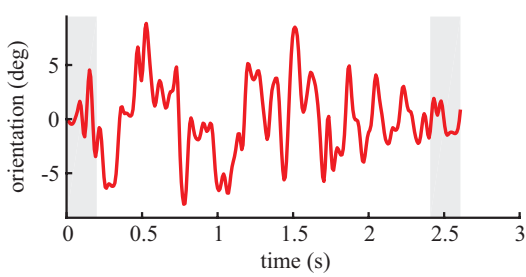

Fig. S7. Tip orientation in the image frame of reference $(\mathrm{A}-\mathrm{C})$ and tip orientation relative to the base path (D-F). Shaded areas, less accurate calculations due to edge effects. (A-C) Black, raw data; orange, fourth-order Butterworth filter, cutoff frequency $18.75 \mathrm{~Hz}$; blue, fourth-order Butterworth filter, cutoff frequency $1 \mathrm{~Hz}$ ('base' orientation). Tip orientation was calculated with respect to the horizontal reference vector in the image frame of reference. Application of Butterworth filters retains general curve characteristics while reducing frame to frame artefacts (orange). (D-F) Red: difference between filtered and base orientation data. The subtraction transforms the data into the ovipositor frame of reference where deviations of the ovipositor tip from the direction of ovipositor insertion can be discerned. Data for the nearly straight insertion is shown in (A) and (D), for curved insertion using the pushing method in (B) and (E), and for curved insertion using the alternating method in $(\mathrm{C})$ and $(\mathrm{F})$.

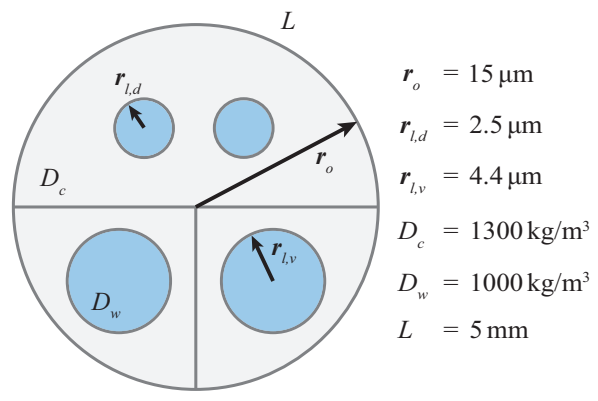

Fig. S8. Schematics of the assumed ovipositor cross-sectional geometry used for force calculations. The shapes and sizes of the ovipositor, valves, and lumina were simplified. Variation at the basal and distal regions of the ovipositor was not taken into account. The ovipositor and lumina were considered to be cylindrical and of equal lengths $(L)$. The dorsal valve was taken as a half cylinder containing two lumina, whereas each of the ventral valves was assumed to be a quarter cylinder containing a single lumen. Lumina were assumed to contain water. Symbols: $r_{0}$, radius of the ovipositor; $r_{l, d}$ and $r_{l, v}$, respective radii of the lumina in the dorsal and ventral valves; $D_{c}$ and $D_{w}$, density of the cuticle and water. 


\section{SI Videos}

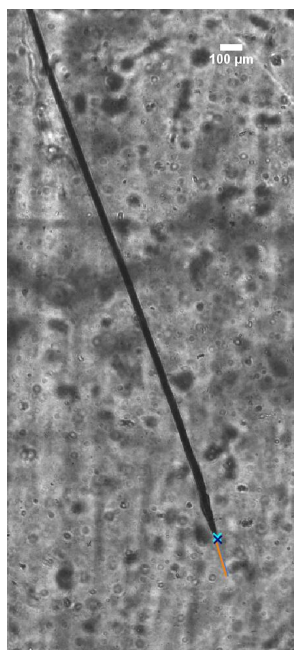

Movie S1. Straight insertion in $2 \%$ gel using both insertion methods. Crosses denote the ends of the dorsal (light blue) and the foremost ventral (dark blue) valves. Lines indicate the base (filtered at $1 \mathrm{~Hz}$ ) and instantaneous (filtered at $18.25 \mathrm{~Hz}$ ) ovipositor orientation in blue and orange, respectively. (Scale bar: $100 \mu \mathrm{m}$.)

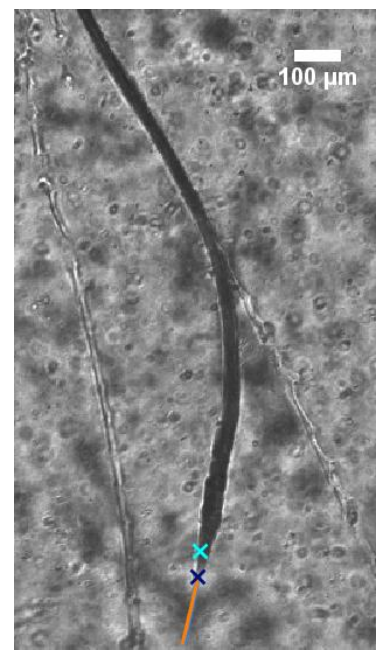

Movie S3. Curved insertion in $4 \%$ gel using the alternating method. Crosses denote the distal ends of the valves, lines the orientation of the ovipositor. Color code is the same as in Fig. S1. (Scale bar: $100 \mu \mathrm{m}$.)

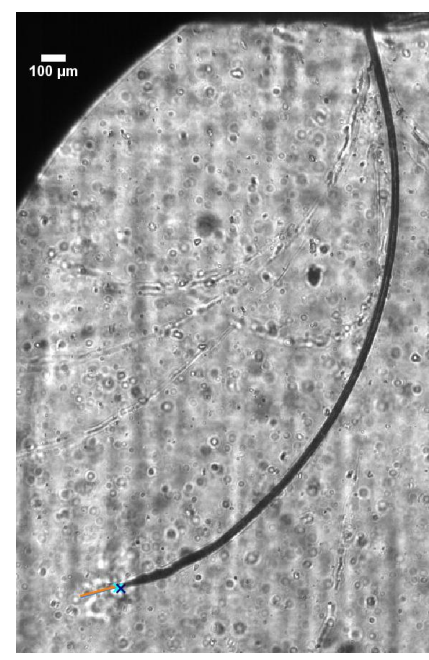

Movie S2. Curved insertion in $2 \%$ gel using the pushing method. Crosses denote the distal ends of the valves, lines denote the orientations. Color code is the same as in Fig. S1. (Scale bar: $100 \mu \mathrm{m}$.) 


$$
1
$$


$\Gamma_{\text {chapter }}$

\section{Stiffness gradients facilitate ovipositor bending and spatial probing control in a parasitic wasp}

Uroš Cerkvenik $^{1}$, Johan L. van Leeuwen ${ }^{1}$, Alexander Kovalev ${ }^{2}$, Stanislav N. Gorb ${ }^{2}$, Yoko Matsumura ${ }^{2}$, and Sander W.S. Gussekloo ${ }^{1}$

${ }^{1}$ Experimental Zoology Group, Department of Animal Sciences,

Wageningen University, De Elst 1, 6708 WD Wageningen, The Netherlands

${ }^{2}$ Zoological Institute: Functional Morphology and Biomechanics, Kiel University, Am Botanischen Garten 9, D - 24118 Kiel, Germany

Submitted 


\section{Abstract}

Many parasitic wasps use slender and steerable ovipositors to lay eggs in hosts hidden in substrates, but it is currently unknown how steering is achieved. The ovipositors generally consist of three longitudinally connected elements, one dorsal and two ventral valves that can slide along each other. For the parasitic wasp Diachasmimorpha longicaudata, it has been shown that protraction of the ventral valves causes incurving of the ventral valves towards the dorsal one, which results in a change in probing direction. We hypothesise that this shape change is due to differences in bending stiffness along the ovipositor. Alignment of the stiff tip of the dorsal valve with a more flexible ventral S-shaped region situated just behind the tip straightens this S-bend and results in upwards rotation of the ventral tip. We show that the S-shaped region of the ventral valves has a low bending stiffness because it contains soft materials such as resilin. In contrast, the large cross-sectional area of the dorsal valve tip area probably results in a high bending stiffness. Elsewhere, the dorsal valve is less stiff than the ventral valves. Our results support the hypothesis that the interaction between the stiff dorsal valve portion and the more flexible S-shaped region co-determine the configurational tip changes required for steering the ovipositor in any desired direction along curved paths in the substrate. This provides novel insights in the understanding of steering mechanisms of the hymenopteran ovipositor, and for the application in man-made probes. 


\section{Introduction}

For many insects it is essential that they can locate and reach resources hidden in substrates. Examples are mosquitoes probing for blood vessels, hemipterans probing for plant vasculature, and parasitic wasps probing for hidden host larvae. Although the drilling mechanism is relatively well understood, it is still uncertain how these animals steer their probes in the desired direction.

Female parasitic wasps use slender, needle-like ovipositors to search for hosts hidden in various substrates. ${ }^{1-3}$ At least some species can actively steer their ovipositors, sometimes in complex trajectories in a single insertion. ${ }^{4-6}$ One of these species, the braconid wasp Diachasmimorpha longicaudata (Fig. 1A), can explore a wide range of the substrate from a single puncture point in any direction with respect to its body orientation, and can achieve highly curved insertion trajectories. ${ }^{4}$ How this and other insects species achieve such curvatures is not completely understood, but for $D$. longicaudata the insertion curvature clearly correlates with configurational changes of the ovipositor.

Like in most wasps, the ovipositor of $\mathrm{D}$. longicaudata consists of multiple elements (valves)—one dorsal and two ventral valves (Fig. 1B, C; Fig. S1) ${ }^{7,8}$ —and has an asymmetric tip in dorsoventral direction. The ventral valve tips bear backwardfacing serrations, while the dorsal valve tip has a prominent bulge. Additionally, the ovipositor is S-shaped proximal to the tip when the valves are aligned (Fig. 1B, Fig. S1). The valves are interconnected with tongue-and-groove linkages (Fig. 1C, Fig. S1) that allow for sliding, but prevent separation of the valves. ${ }^{9,10}$ Generally, the valves slide back and forth during probing in stiff materials, ${ }^{4}$ which is thought to reduce the overall net pushing force on the ovipositor in the process and limits the risk of buckling. ${ }^{10}$

Kinematic analysis of probing behaviour of $D$. longicaudata, showed that insertion with predominant protrusion of the ventral valves leads to curved trajectories. Upon their protraction, the ventral valves curve towards the dorsal side of the ovipositor (Fig. 1D, Chapter 4). ${ }^{4}$ The dorsal curving of the ventral valves upon their protraction is also observed outside a substrate, which implies that it occurs without external forces and is thus initiated from within the ovipositor (Fig. S1). In contrast, the dorsal valve remains approximately straight when it is protracted inside a substrate (Fig. 1D, Chapter 4). ${ }^{4}$ Additional to the changes in the very tip of the ovipositor, protraction of either the dorsal or the ventral valves results in a straightening of the S-bend region just proximal of the tip (Chapter 4, Chapter 4$)$. 


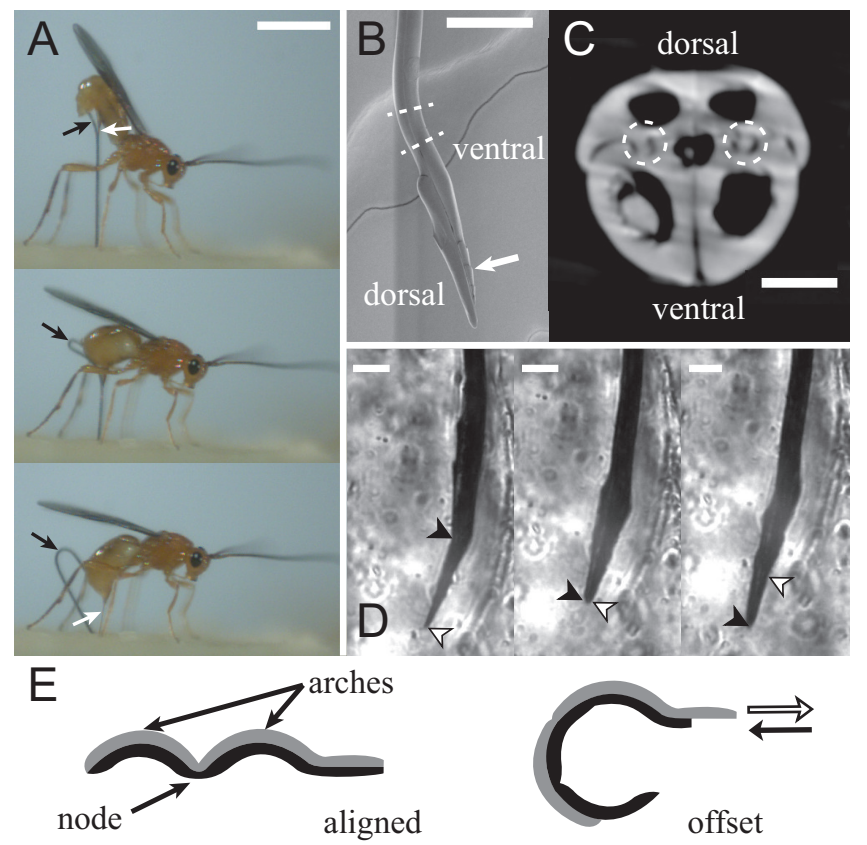

Fig. 1. Wasps ovipositor morphology and kinematics. (A) Ovipositor insertion by D. longicaudata. Top: the ovipositor (white arrow) is positioned vertically while supported by the sheaths (black arrow) before insertion. Middle: during insertion, the sheaths peel away from the ovipositor at its base. Bottom: at full insertion the sheaths completely detach. Scale bar $5 \mathrm{~mm}$. (B) SEM image of the ovipositor tip-side view; white arrow indicates serrations on the ventral valves. dashed lines indicate the position of the cross section shown in (C). Scale bar $100 \mu \mathrm{m}$. (C) Ovipositor cross-section (see (B)) showing the dorsal valve (top) and two ventral valves (bottom), dashed circles indicate the tongue-and-groove connection. Scale bar $10 \mu \mathrm{m}$. (D) Ventral valves (white arrow heads) curve towards the dorsal valve (black arrow heads) upon protraction. No such curving is observed when the dorsal valve is protracted. Scale bar $50 \mu \mathrm{m}$. (E) Proposed bending principle in ovipositors with differential valve sclerotization. ${ }^{11}$ Left: when the highly sclerotized and therefore stiff arches align the ovipositor is characteristically arched and 'straight' at a larger spatial scale. Right: Upon protraction of the ventral valves the arches align with the less sclerotized softer nodal regions and the ovipositor bends. (A-D) modified from [4].

Because all the muscles that operate the valves are located in the animal's abdomen, $8,12,13$ we expect that the shape and curvature changes of the ovipositor tip occur only due to longitudinal shifts of the valves with respect to one another. It is therefore likely that the valve structural and material properties play an important role in such configurational changes, although external forces may enhance the overall effect.

One possible mechanism of induced bending has been described for parasitoids of genera Zaglyptogastra and Pristomerus and relies on the differential sclerotization of the valves (Fig. 1E). ${ }^{11}$ These wasps have ovipositors characterized by a series of intermittent arches and nodes near the tip. ${ }^{14,15}$ The arches are regions of sclerotized cuticle and are presumably stiffer than the nodes in between. ${ }^{11}$ When the valves are aligned at their tips, arches and nodes of the dorsal and ventral valves also align. Protraction of one of the valves aligns the arches with the nodes on the other valve. 
This causes bending of the ovipositor as the nodes in each of the valves conform to the shape of the arches of their adjacent valves. This mechanism can lead to bending of up to $180^{\circ}$, but is considered to be unsuitable for piercing hard substrates, which implies that these wasps only exploit existing holes in the substrate to access their hosts. ${ }^{11}$

Based on the anatomical characteristics of the ovipositor in D. longicaudata, and specifically the S-shaped region and the bulge on the dorsal valve, it is plausible that these wasps rely on a steering mechanism similar to the one described above. In the case of $D$. longicaudata, the thickened portion of the dorsal valve might act as a stiff region and the S-shaped region as a deformable soft region. The stiff region will probably be highly sclerotized, whereas the parts undergoing deformations, such as the S-shaped region, are exposed to repetitive strains and are therefore expected to be adapted to minimize potential damage. A good candidate for mitigating harmful effects of loading in insect cuticle is resilin, an elastic protein that can undergo considerable strains without damage. ${ }^{16}$ Resilin is commonly found in highly deformable and high-load bearing structures such as jumping legs of locusts and fleas, ${ }^{17,18}$ wings of beetles and dragonflies, ${ }^{19,20}$ beetle tarsal setae, ${ }^{21}$ and sound producing organs of cicadas. ${ }^{22}$

We hypothesise that the ovipositor of $D$. longicaudata exhibits a stiff region near the bulge of the dorsal valve, probably due to high sclerotization combined with its specific geometry, and a flexible zone in the observed S-curve consisting of more flexible material and geometry. Alignment of these regions would result in the dorsal curving of the tips and the straightening of the S-bend region. To determine if stiffness variations in the ovipositor of $D$. longicaudata are present, we measured the bending stiffness along its length with small scale three point bending tests. This measured bending stiffness is determined by the shape (structure) of the ovipositor and its material properties. The second moment of area along the ovipositor length, describing the structural bending stiffness, was obtained from histological cross-sections and high-resolution Synchrotron X-ray microtomographs. The material composition of the ovipositor was qualitatively determined with confocal laser scanning microscopy (CLSM), and estimated by dividing the bending stiffness by the cross-sectional area. This gave us insight in the distribution of bending stiffness along the ovipositor of D. longicaudata and confirmed that there is a mechanism of flexible and stiff zones which can be aligned to induce flexion. This provides a better understanding of how steering of probes can be achieved, and how it contributes to the probing versatility of parasitic wasps and potentially other probing insects. In addition, these findings also indicate how steering without intrinsic motors can be achieved in man-made probes. 


\section{Results}

\section{Gross anatomy of the ovipositor}

The ovipositor of $D$. longicaudata is a slender structure that connects to the musculoskeletal apparatus at its base (Fig. 2A). ${ }^{12}$ The three valves it consists of form a cylindrical structure approximately circular in cross-section, which encloses the egg canal (Fig. 2B, C). The unloaded shaft of the ovipositor is slightly curved, making it dorsally concave. An S-shaped region is present just proximal to the distinct ovipositor tip (Fig. 2A (inset), Fig. 2C). For most of its length, the dorsal valve is crescent-shaped in cross section, while the ventral valve cross-sections resemble a quarter of an ellipse (Fig. 2B, C). The dorsal valve connects to each ventral valve with a tongue-and-groove linkage that is 'mushroom-shaped' in cross-section (Fig. 2B).

The cross-sectional area of the ovipositor varies little along most of its length (similar to Fig. 2B2), except for its base (Fig. 2B1) and the morphologically distinct tip (Fig. 2B3-B5). The base of the ovipositor is enlarged and polygonal in cross-section. While the dorsal valve base is approximately five times wider than most of the ovipositor shaft, the ventral valves are only slightly enlarged compared to their cross-sectional size along the shaft (Fig. 2B1). At the tip, distal to the S-shaped region, the dorsal and ventral valves both possess a bulging area before tapering towards the vertex (i.e. the point furthest away from the base). Additionally, the crosssectional area of the tapered tips changes shape towards the tip vertices. The bulge on the dorsal valve changes from rectangular to oval, while that of the ventral valves changes from approximately oval to approximately triangular (Fig. 2B4-B5).

\section{Structural properties}

We determined the structural resistance to bending of the ovipositor and individual valves, by calculating the second moment of area in dorsoventral direction $\left(I_{\mathrm{x}}\right)$, because this is the main direction of bending observed in vivo. ${ }^{4}$ This second moment of area varied along the ovipositor length (for full comparison and statistics see Table S1) in accordance with the above described anatomy (Fig. 2A-B, Fig. 3A). The $I_{\mathrm{x}}$ of both the whole ovipositor $\left(I_{x, 0 v}\right.$; grey data in Fig. $\left.3 \mathrm{~A}\right)$ and the dorsal valve $\left(I_{x, d v}\right.$; light blue data in Fig. 3A) sharply decreased from the base to the shaft, varied little along the shaft, and sharply increased at the location of the bulge of the dorsal valve, which was followed by a sharp decrease towards its vertex. To illustrate, at the base (at $6.9 \%$ of the ovipositor length $(\mathrm{OL})$ ), $I_{\mathrm{x}, \mathrm{ov}}$ was $12.8 \times 10^{4} \mu \mathrm{m}^{4}$, which diminished to about one quarter of this value along the shaft (lowest values 

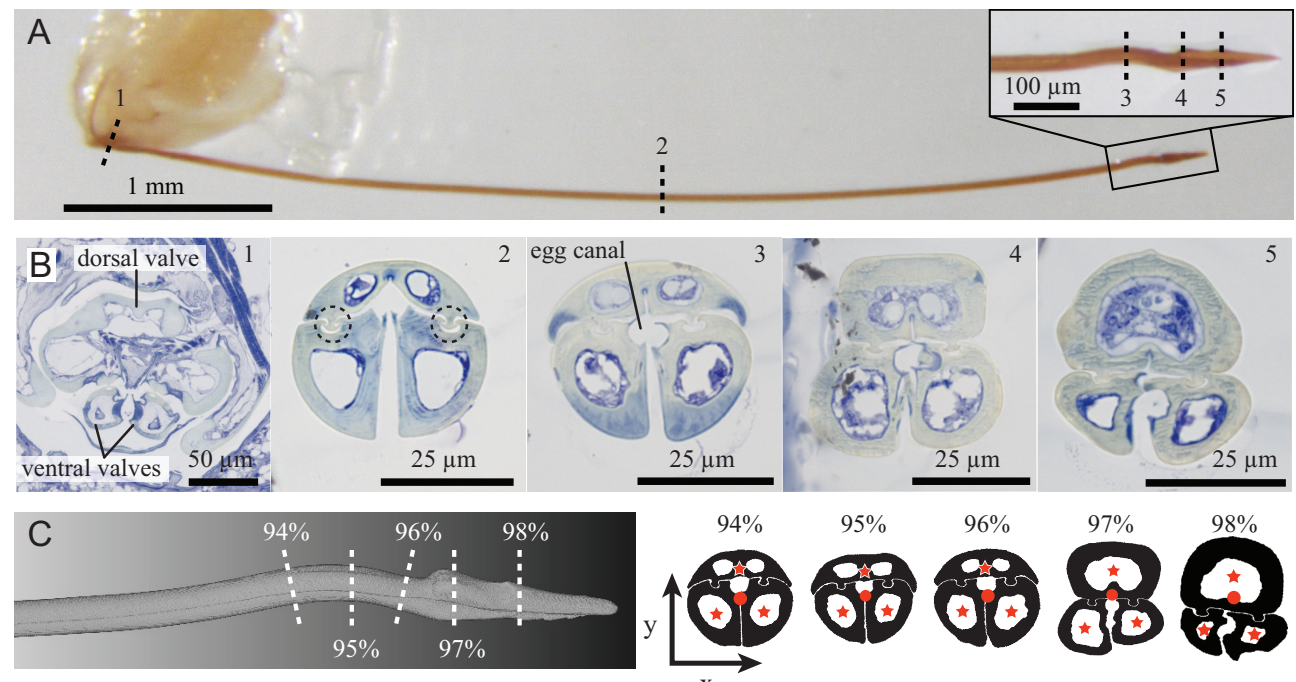

Fig. 2. Gross anatomy of the ovipositor. (A) Lateral view of a dissected ovipositor of D. longicaudata with parts of its basal plates that are involved in actuation (left side). Dorsal valve is on the top, ventral valves on the bottom. Inset-close up of the distinct tip morphology: the S-bend region (3) and the bulge of the dorsal valve (4). (B) Histological cross-sections of the ovipositor at locations shown in (A), stained for proteins (blue). Dorsal side is up. The tongue-and-groove mechanism is indicated with black, dashed circles in (2). (C) 3D reconstruction of the high-resolution $\mu \mathrm{CT}$ scan of the ovipositor tip with minimal cross-sections calculated at indicated locations. The dorsal side is up. The centroids used in calculations of the second moments of area (in Cartesian coordinate system $x, y$ ) are indicated in the cross-sections: red dots for whole ovipositor, red stars for individual valves.

around $3.64 \times 10^{4} \mu \mathrm{m}^{4}$ at $66 \% \mathrm{OL}$ ), and increased at the bulge on the ovipositor tip (highest value of $19.93 \times 10^{4} \mu^{4}$ at $96.8 \%$ OL), before decreasing to 0 at the end of the tip. The extreme values for the dorsal valve $\left(I_{x, \mathrm{dv}}\right)$ occurred at comparable locations, showed a similar pattern, but were smaller $\left(4.51 \times 10^{4} \mu^{4}\right.$ at $6.9 \% \mathrm{OL}$, $0.14 \times 10^{4} \mu \mathrm{m}^{4}$ at $68 \% \mathrm{OL}$, and $3.06 \times 10^{4} \mu \mathrm{m}^{4}$ at $\left.96.9 \% \mathrm{OL}\right)$.

The $I_{\mathrm{x}}$ of the ventral valves $\left(I_{\mathrm{x}, \mathrm{vv}}\right)$ varied little along their entire shafts and increased only at their tip (dark blue data in Fig. 3A). The values ranged from $0.60 \times 10^{4} \mu^{4}$ at $6.9 \%$ OL to $0.80 \times 10^{4} \mu \mathrm{m} 4$ at $63.6 \% \mathrm{OL}$, and were with $1.61 \times 10^{4} \mu \mathrm{m}^{4}$ highest at $96.7 \%$ OL. The dorsal valve was in the dorsoventral direction thinner than the ventral ones along most of their length. Only where the base broadens $I_{\mathrm{x}, \mathrm{dv}}$ was higher than $I_{x, v v}(<10.5 \%$ OL; Fig. 3A, left) and at the bulge on the tip ( $>96.6 \%$ OL; Fig. 3A, right).

The contribution of the individual valves to the second moment of area of the complete ovipositor (i.e. when computed with respect to the centroid obtained from whole ovipositor cross-sections; see Fig. S2) were considerably higher than the values given here, but showed a similar pattern. Here we show the data with respect to the centroid of the object of interest, because they link directly to the bending stiffness measured in the three point bending experiments discussed below. 

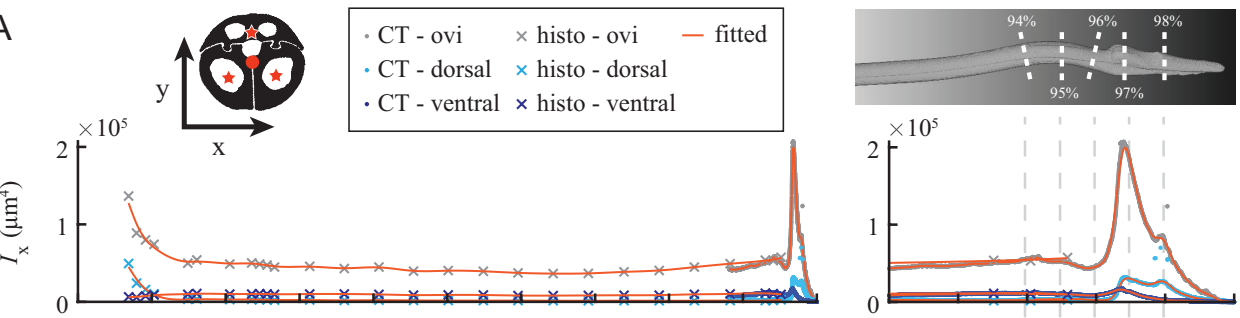

B
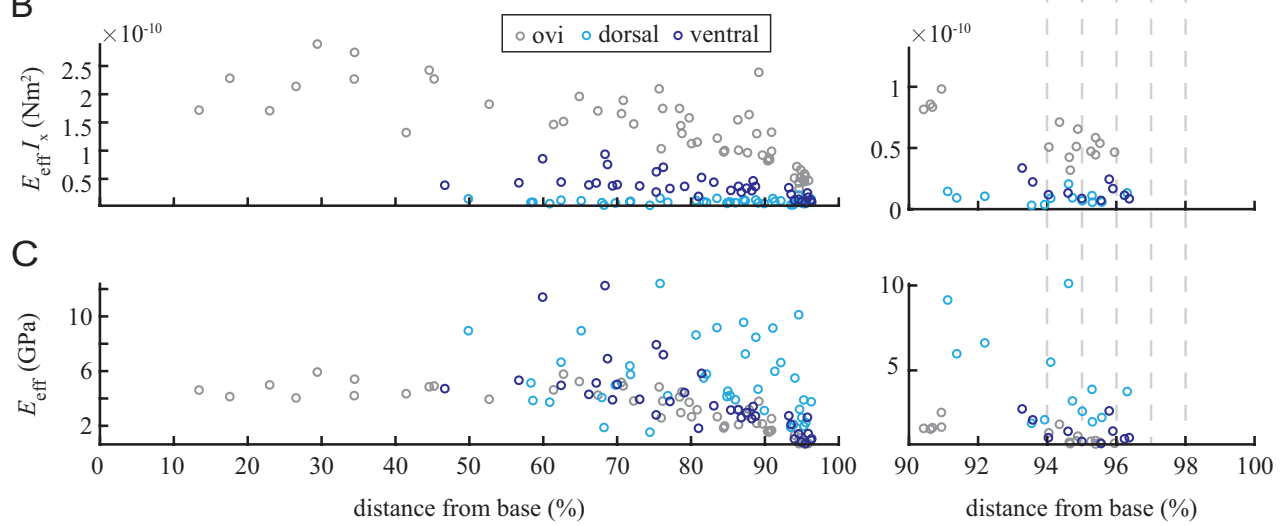

Fig. 3. Ovipositor geometry, bending stiffness, and effective modulus of elasticity vary along its length. Left: values along most of the ovipositor length; right: a close up of the distal region of the ovipositor. (A) The second moment of area around the $x$-axis $\left(I_{\mathrm{x}}\right)$ changes little along most of the ovipositor length. Grey: whole ovipositor; dark blue: ventral valve (mean of both elements); light blue: dorsal valve; orange lines: fitted cubic smoothing spline (smoothing parameters 0.98 and 0.5 were used for the $\mu \mathrm{CT}$ and histological data, respectively). Crosses: histological data, dots $\mu \mathrm{CT}$ data. The two types of data were scaled and then combined (see $S I)$. (B) Bending stiffness $\left(E_{\text {eff }} I_{\mathrm{x}}\right)$ of the ovipositor and the ventral valves decreases in the distal direction and is lowest in the pre-bent S-shaped region. The $E_{\text {eff }} I_{\mathrm{x}}$ of the dorsal valve varies little along its length. (C) The effective material stiffness $\left(E_{\text {eff }}\right)$ of the whole ovipositor and individual valves is smallest in the S-bend region than in the rest of the ovipositor.

\section{Bending stiffness}

Bending stiffness $\left(E_{\mathrm{eff}} I_{\mathrm{x}}\right)$ over the length of different parts of the ovipositor was obtained with three point bending experiments, in which one end of the sample was clamped. Two thin razorblades were moved upward while a force transducer fixed to a third razorblade, centred between the other two, measured the reaction force (see materials and methods for details). We were not able to measure the stiffness of the tip, because it was too short to cover the three point bending setup. All values recorded at $1.87 \mathrm{~mm}$ from the clamp or closer were omitted from the analysis based on the estimated effect of the clamp using Finite Element Model studies (FEM, see $S I)$. Additionally, we also excluded some measurements because twisting of the samples occurred during the bending tests, which resulted in erroneous force estimates. Values for bending stiffness reported here (for full comparison and statistics see Table S2) are all determined for curvatures within the natural observed range (see $S I)$. 
The shaft $(<93 \%$ OL) of individual ventral valves were stiffer than the dorsal one (compare dark and light blue points in Fig. 3B). The mean recorded stiffness of the shaft of the whole ovipositor $\left(15.72 \times 10^{-11} \mathrm{~N} \mathrm{~m}^{2}\right)$ was approximately 3.6 times higher than the mean shaft stiffness of the isolated ventral valves $\left(4.40 \times 10^{-11} \mathrm{~N} \mathrm{~m}^{2}\right)$ and more than 17 times higher than the mean stiffness of the shaft of the isolated dorsal valve $\left(0.9 \times 10^{-11} \mathrm{~N} \mathrm{~m}^{2}\right)$.

The stiffness of both the ovipositor and the ventral valves was significantly lower in the S-shaped region between 93 and 96\% OL (Table S2). The mean recorded value in the S-shaped region for the whole ovipositors $\left(5.12 \times 10^{-11} \mathrm{Nm}^{2}\right)$ is approximately a third $(30.8 \%)$ of the mean value of the shaft. Similarly, the stiffness of the S-shaped region of the isolated ventral valves $\left(1.57 \times 10^{-11} \mathrm{~N} \mathrm{~m}^{2}\right)$ is also approximately a third $(28.0 \%)$ of the mean stiffness of their shafts. Finite Element Model (FEM) studies (see SI) showed that a S-shape morphology compared to a straight beam resulted in only a $7 \%$ reduction in measured reaction forces in a three point bending experiment, indicating that the differences in stiffness between the shaft and S-bend region cannot be explained by the S-bend shape alone.

The variation in the dorsal valve data is high and we did not observe significant differences between the shaft and the S-shaped region $\left(0.90 \times 10^{-11}\right.$ vs $0.88 \times 10^{-11}$, Table S2). This variation is caused at least partially by the limited accuracy of the force transducer (see Materials and methods).

\section{Material properties}

\section{Effective moduli of elasticity}

The bending stiffness measured in the three point bending experiments depends on the second moment of area and the material properties of the ovipositor and the valves. We were able to estimate the material properties of these structures and express them as the effective modulus of elasticity $\left(E_{\mathrm{eff}}\right)$ by dividing the bending stiffness by the second moment of area. The effective modulus of elasticity showed the same variation along the length of the ovipositor as seen for the bending stiffness (Fig. 3C). The effective elasticity of the ovipositor, ventral, and dorsal valves is significantly lower in the S-shaped region than in the shaft (Table S2). For the whole ovipositor the effective modulus of elasticity of the S-shaped region is almost 4 times lower than the shaft $\left(0.92 \times 10^{-11}\right.$ vs $\left.3.60 \times 10^{-11}\right)$, over 3 times lower in the ventral valves $\left(1.46 \times 10^{-11}\right.$ vs $\left.4.81 \times 10^{-11}\right)$, and only 1.6 times lower in the dorsal valve $\left(3.70 \times 10^{-11}\right.$ vs $\left.5.95 \times 10^{-11}\right)$. 


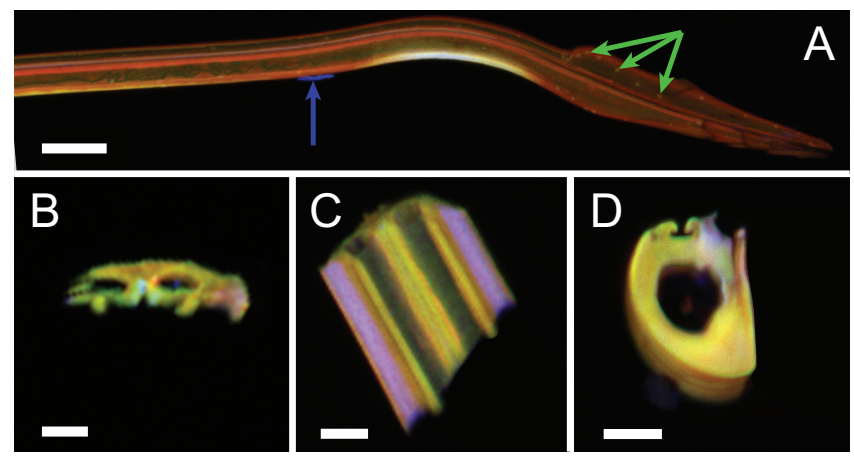

Fig. 4. Qualitative analysis of material composition of insect cuticle using its autofluorescent properties. The blue channel ( $405 \mathrm{~nm}$ excitation) indicates resilin. The green ( $488 \mathrm{~nm}$ excitation) and red channel (555 and $639 \mathrm{~nm}$ excitation) indicate well sclerotized cuticle. (A) The distal part of the ovipositor showing high resilin presence in the ventral part of the S-shaped region-top: dorsal valve; bottom: ventral valve (see also Fig. S3). Green dots are most likely sensors (green arrows). Blue arrow marks dirt on the surface of the ovipositor. (B, C) Cross-section (B) and ventral aspect (C) of parts of the dorsal valve from the S-shaped region, showing resilin near the egg canal and in the lateral edge. (D) Cross-section through the ventral valve proximal to the $S$-shaped region showing resilin presence in the wall of the egg canal. Scale bars: $50 \mu \mathrm{m}(\mathrm{A}), 10 \mu \mathrm{m}(\mathrm{B}-\mathrm{D})$. Images were cropped and optimized for the shown structures.

\section{Qualitative assessment of ovipositor material composition}

To link the differences in effective modulus of elasticity to actual differences in material properties we analysed the autofluorescence of the ovipositor in a confocal laser scanning microscope (Fig. 4, Fig. S3). The green (488 nm excitation) and red (555 and $639 \mathrm{~nm}$ excitation) autofluorescence signals indicate that the cuticle of the ovipositor is well sclerotized. The more flexible S-shaped region of the ventral valves is clearly less well-sclerotized and shows high levels of resilin (blue channel; $405 \mathrm{~nm}$ excitation; Fig. 4A). Other regions with high resilin content and less sclerotized cuticle are the most lateral parts of the dorsal valve (Fig. $4 \mathrm{C}$ ) and the valve inner walls forming the egg canal (Fig. 4B, D).

\section{Discussion}

Parasitic wasps, mosquitoes and hemipterans are capable of inserting probes into a variety of substrates, and even explore the substrate by steering their probes in a range of directions. ${ }^{4,23,24}$ Hitherto, very little was known about how these animals are able to induce a shift of the probing direction. The kinematic analysis of ovipositor insertions in the parasitic wasp D. longicaudata indicates that the reciprocal sliding movements of its valves may be important in steering. During probing, reciprocal movements are presumed to reduce the required net pushing forces on the probe and thus limit or avoid its buckling. ${ }^{4,10}$ Additionally, in D. longicaudata, the protraction of the ventral valves over the dorsal one induces a clear shape change in the ovipositor tip that leads to curved insertion trajectories (Chapter 4). ${ }^{4}$ 
We hypothesise that the ovipositor of $D$. longicaudata employs the same principle of differentially sclerotized (and therefore differentially stiff) valves as suggested for the arched ovipositors of Zaglyptogastra sp. and Pristomerus sp. ${ }^{11}$ For this mechanism to work both soft and very stiff regions must be present. Analysis of the bending stiffness of the $D$. longicaudata ovipositor as a whole indeed showed an approximately $67 \%$ lower bending stiffness in the S-shaped region than in the rest of the shaft. This reduction in stiffness is mainly caused by a change in material properties (effective modulus of elasticity). Structural properties even increase the stiffness as can be seen from the increased second moment of area in the S-bend region (Table S1). The reduced stiffness can neither be explained by the curved morphology as this only results in a 7\% decrease in stiffness compared to a straight morphology with the same material (see $S I$ ). That the S-shaped region is composed of softer materials than other regions along the ovipositor is substantiated by the CLSM images, which show a stronger fluorescence signal indicative for the presence of flexible resilin at that location.

The derived effective moduli of elasticity of the ovipositor and its valves in D. longicaudata (Fig. 3C, Table S2) are in agreement with those previously estimated for other ovipositors or the intromittent organs of beetles which range from 0.6 to $10 \mathrm{GPa} .{ }^{10,25,26}$ The effective elasticity of the whole ovipositor reported above are presumably influenced (reduced) by the possible sliding of the valves along each other during the three point bending experiments. The effect of sliding valves was, however, not estimated in this study.

Although areas of increased stiffness have not been directly measured, it can be assumed that the tip of the ovipositor is stiffer than the rest of the ovipositor. We observed a dramatic increase in second moment of area of the whole ovipositor at the location of the dorsal bulge, where the mean second moment of area was two times higher than in the shaft (Table S1, Figs 2, 3A). Because the autofluorescence of the materials of the shaft and the tip did not differ, we can assume that material properties are the same, and that the twofold increase of second moment of area at the bulge results in a twofold increase in bending stiffness of the ovipositor. This is mainly due to the bulge in the dorsal valve, which has a six times higher second moment of area than the dorsal valve shaft. The slight thickening of the ventral valve at the same location only leads to a very small increase in second moment of area (Table S1).

According to the steering hypothesis described above, alignment of flexible and stiff areas should lead to configurational changes of the ovipositor distal end. In the ovipositor of D. longicaudata, this occurs when the S-shaped region of either the dorsal or the ventral valve aligns with the morphologically distinct tip of the 


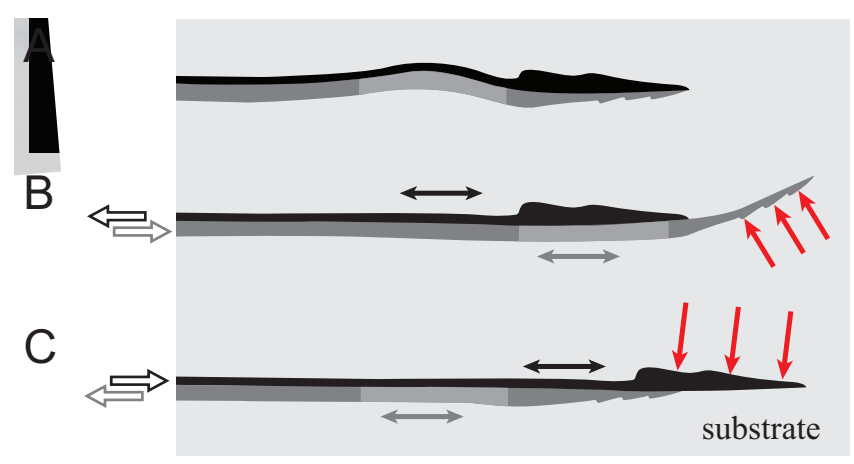

Fig. 5. Proposed steering mechanism in D. longicaudata. (A) Ovipositor with aligned valves (black: dorsal; dark grey: ventral) shows a straight shaft, the adjacent S-shaped region, and the tip. (B) Protraction of the ventral valves (movement indicated by open arrows) straightens the less rigid S-shaped region (light grey section) as it aligns with the stiff tip of the dorsal valve, and results in dorsal curving of the ventral valve tips. Bending of the ventral valves is probably enhanced by the asymmetrical substrate forces acting on the bevelled tip (red arrows). (C) Protraction of the dorsal valve straightens its S-shaped region as it aligns with the ventral valve tips. The S-shaped region of the ventral valves is also straightened which might potentially lead to dorsal curving. However, the bevelled tip of the dorsal valve probably induces asymmetric reaction forces from the substrate that counteract these bending moments and thus a straight protraction is observed.

opposite valve. The S-shaped region and the bulge area of the tip have approximately the same length ( $3 \%$ OL, Figs 1B, 3A; Table S1), which facilitates an accurate alignment. Below, we describe how the alignment of the different parts may lead to these configurational changes, and consequently to a change in probing direction. We consider two valve configurations: (i) protraction of the ventral valves, and (ii) protraction of the dorsal valve (Fig. 5).

Protraction of the ventral valves moves them from their rest position (Fig. 5A) forward, such that their less stiff S-shaped regions align with the stiff bulge of the dorsal valve tip (Fig. 1). At the same time, the dorsal S-shaped region aligns with the shaft of the ventral valves. Because the dorsal valve bulge is much stiffer than the S-shaped region of the ventral valves, the S-shaped region of the ventral valves straightens as observed in [4] and shown in Chapter 4. The S-shaped region of the dorsal valve is presumably straightened because the shafts of the ventral valve are stiffer than the S-shaped region of the dorsal valve (Fig. 3B, Table S2). We expect that after straightening of the S-shaped region the natural angle between the $\mathrm{S}$-shaped region and the tip region remains, which causes the dorsal curving of the ovipositor (Fig. 5A, B) as previously observed. ${ }^{4}$ While probing in stiff substrates, the substrate reaction forces presumably prevent the bending of the long ovipositor shaft, but allow for the curving of the short tip. Additionally, the bevel shape of the ventral valve tips (Figs 1, 5B) generates asymmetric substrate reaction forces that potentially enhance the curving of the tip during insertion of the ovipositor, and may explain the high curvatures observed during probing. ${ }^{4}$ 
Although this pattern seems straightforward, it is difficult to predict what happens when only a single ventral valve is protracted. The resulting bending moments on the ovipositor tip would in this case be asymmetric and could lead to local twisting of the valves. However, this is hard to substantiate experimentally and is outside the scope of this paper.

Dorsal valve protraction aligns the relatively soft S-shaped region of the dorsal valve with the stiffer ventral valve tips, which also possess a small bulge near their tips (Fig. 5C, Table S1). This presumably straightens the S-shaped region of the dorsal valve. The ventral S-shaped region in this case aligns with the shaft of the dorsal valve, and also straightens out (Figs 1D, 5C). ${ }^{4}$ The straightening of both S-shaped regions should lead to dorsal curving of the ovipositor tip as explained above. This does occur when the ovipositor is outside a substrate (Chapter 4) but was not observed during natural probing (Chapter 4$).{ }^{4}$ We hypothesise that substrate reaction forces acting on the bevelled dorsal valve tip counteract any dorsal bending moments originating from the straightening of the S-shaped regions. Protraction of the dorsal valve inside a substrate therefore results in an approximately straight path, but in dorsal curving of the tip outside the substrate. Because the forces acting on the bevel counteract the bending moments within the ovipositor, we expect that protracting the dorsal valve by more than its tip length leads to ventral curving. However, such big protraction amplitudes seem to be outside the natural probing range. 4

In both ventral and dorsal valve protraction, the S-shaped region of the ovipositor undergoes continuous deformations and is therefore subjected to repetitive strains, which could lead to damage due to material failure. The strains are larger in the thicker and stiffer ventral valves, compared to the relatively thin and flexible dorsal valve. The expected strains in the ventral valves might induce damage to their outer walls, which seems to be avoided by the presence of resilin (Fig. 4A). Resilin is highly resistant to damage at large deformations and presumably makes the sclerotized cuticle tougher, reducing the likelihood of damage as has been previously proposed for beetle wings. ${ }^{19}$

Interestingly, resilin-containing regions were also found in the inner walls of the valves around the egg canal and in the lateral sides of the dorsal valve (4B-D). These softer central regions could facilitate the passage of 'oversized' eggs during egg laying process. ${ }^{27-31}$ Additionally, having softer lateral sides could be important in the wasps capability of exploring the substrate in an approximately half ellipsoid without rotating its body, ${ }^{4}$ by facilitating lateral bending or twisting of the ovipositor. 
Although the functional principles of ovipositor bending are similar between $D$. longicaudata, Zaglyptogatra sp. and Pristomerus sp., the implementation of the mechanism in $D$. longicaudata differs from the other two species. Namely, the ovipositors of Zaglyptogastra and Pristomerus possess a series of stiff (arches) and flexible (nodes) regions, whereas the ovipositor of $D$. longicaudata has only a single flexible area near its tip. This difference might be linked to the fact that $D$. longicaudata probes in solid substrates, while Zaglyptogastra and Pristomerus cannot. ${ }^{11}$

D. longicaudata probes in solid substrates such as citrus fruits. ${ }^{4,32}$ The modulus of elasticity of orange peels estimated for freshly harvested fruits ranges between 3.5 and $4.3 \mathrm{GPa} .{ }^{33}$ It is likely that fruits infested with fruit-fly larvae are softer, although the stiffness of such fruits is not known. Nevertheless, D. longicaudata is capable of puncturing artificial gels with the stiffness of at least $0.2 \mathrm{GPa} .{ }^{4}$ We argue that this is presumably facilitated by the lower number of stiff and flexible regions in the ovipositors of D. longicaudata compared to the ovipositors found in Pristomerus or Zaglyptogastra. Although the flexible S-shaped region in the ovipositor of D. longicaudata may still bend when axial loading is applied, small offsetting of the valves potentially strengthens this region without inducing strong ovipositor curvatures that would hinder puncturing. The low number of flexible and stiff nodes might come at a price as it is likely that the S-shaped ovipositors cannot bend as much as the arched ones. We therefore hypothesise that the ovipositor S-shaped region is a morphological adaptation for probing and steering in solid substrates such as fruits that occurs in D. longicaudata and closely related species. ${ }^{34,35}$

Here we show that the structural and material properties of the ovipositor vary across its individual elements. This, together with the kinematics of individual elements, ${ }^{4}$ leads to controlled shape changes of the probe tip and therefore offers a degree of control over spatial probing of the animal. The probing principles found in parasitic wasps can be presumably also applied to other insect probers including mosquitoes and hemipterans. These animals also explore a variety of substrates with multi-element probes ${ }^{36,37}$ that are moved reciprocally during substrate exploration. ${ }^{23,38}$ This kinematics indicates that these insects use similar principles of buckling avoidance and steering as described for parasitic wasps. It would be interesting to see if similar steering mechanism as described here can be also found in other insect groups. This would add to our understanding of the convergent evolution that led to the widespread usage of slender, multi-element probes capable of independent and reciprocal element movements and steering. Furthermore, it also indicates that such probes are highly effective for substrate exploration and studying their working principles may be also beneficial for the design of slender man-made probes. 
Slender, multi-element probes inspired by parasitic wasp ovipositors have been developed and can be steered without placing actuators inside the probe and without axial rotation. ${ }^{39,40}$ The individual elements of such probes slide along each other and therefore form an adjustable bevel tip. ${ }^{40,41}$ The probe is steered by taking advantage of the substrate reaction forces acting on the adjustable bevel. The amount of curving in these probes is heavily dependent on the material properties of the substrate and the probe. The steering mechanism described here is not dependent on the substrate interaction, although it can be enhanced by it. Therefore, application of a mechanism using stiff and deformable regions as described here will result in probes less susceptible to substrate differences. The steering mechanism can be implemented by adjusting either the material properties or the cross-sectional area of the probe S-shaped region which would not compromise the diameter of the probe. Such design would therefore propel further miniaturization of man-made probes.

\section{Material and methods}

\section{Animals}

Animals were taken from the breeding colony at the Experimental Zoology Group at Wageningen University. The rearing protocol was the same as described in [4]. All animals were anaesthetized with $\mathrm{CO}_{2}$ and then decapitated immediately prior to fixation or experimentation.

\section{Structural properties}

\section{Histology}

For histological sectioning, the abdomen with the intact ovipositor was dissected from the body and fixed with a mixture of $0.1 \mathrm{M}$ sodium cacodylate buffer (dimethylarsenic acid sodium salt trihydrate; Merck, Germany), 2\% (weight/volume) paraformaldehyde (Merck, Germany), and 2.5\% (volume/volume) glutaraldehyde (EMS, PA, USA) on ice $\left(0^{\circ} \mathrm{C}\right)$ for $2.5 \mathrm{~h}$. The sample was washed with the $0.1 \mathrm{M}$ cacodylate buffer twice at $0^{\circ} \mathrm{C}$, dehydrated in a series of ethanol solutions of increasing concentration from $50 \%$ to $100 \%$, and transferred to epoxy resin (epon; Embed-812 Embedding Kit, EMS, PA, USA) through a sequence of solutions: a mixture of ethanol and propylene oxide (PO), pure $\mathrm{PO}$, a series of 3:1 and 1:1 mixtures of $\mathrm{PO}$ and epon, and finally pure epon. The samples in epon were put in moulds and left to harden overnight at $60-65^{\circ} \mathrm{C}$. 
Semi-thin sections of $1 \mu \mathrm{m}$ and $2 \mu \mathrm{m}$ thickness were cut using a Reichert Ultracut $S$ ultramicrotome (Leica Microsystems $\mathrm{GmbH}$, Germany) and stained with a mixture of $1 \%$ Toluidine blue (VWR, PA, USA) and 1\% Borax (disodium tetraborate decahydrate; Merck, Germany) dissolved in distilled water. The sections were photographed with a Leica DFC $450 \mathrm{C}$ camera (5MP) mounted on a Leica DM6b microscope (Leica Microsystems) with $40 \times$ or $100 \times$ objective. The images were stored as tiff files for further analysis (see below).

\section{High-resolution $\mu C T$ scan}

We removed the ovipositor from a single collection-specimen of D. longicaudata obtained from the Naturalis Biodiversity Center (Leiden, Netherlands) and mounted it on a pin using beeswax such that it could rotate around its longitudinal axis.

We scanned $\sim 600 \mu \mathrm{m}$ of the distal part of the ovipositor in two consecutive scans with $30 \mu \mathrm{m}$ overlap at the TOMCAT beamline X02DA of the Swiss Light Source (Paul Scherrer Institute, Switzerland) with a $14 \mathrm{keV}$ monochromatic X-ray beam. Projection images were recorded over an angular range of $180^{\circ}$ with an angular step of $0.1^{\circ}$ with a PCO Edge 5.5 sCMOS camera (exposure time of $100 \mathrm{~ms}$ ), using a $5 \mu \mathrm{m}$ thick FEE Tb:LSO scintillator and an optical magnification of $40 \times$, resulting in an effective pixel width of $162.5 \mathrm{~nm}$ at a propagation distance of $9 \mathrm{~mm}$. The scans were reconstructed using the gridrec reconstruction algorithm [42] in conjunction with propagation-based phase contrast $(\delta / \beta=100)$ as described by [43].

\section{Analysis of the cross-sections}

Seg3D 2.2.1 (University of Utah) ${ }^{44}$ was used for segmentation of the reconstructed CT slices and histological images. Manually adapted thresholds were applied to roughly isolate the exoskeleton from the background. Masks were further manually adjusted, converted to binary images, and rotated such that the dorsal valve was on top (e.g. Fig. 2C). Histological sections were aligned manually, while the $\mu \mathrm{CT}$ slices were rotated by the average angle $\left(169.66^{\circ} \pm 3.68^{\circ}\right)$ measured from 16 slices spread equally along the length of the dataset.

To obtain more accurate estimates of the second moment of area, we recalculated the cross-sections perpendicular to the central axis along the full length of the $\mu \mathrm{CT}$ scan. The points along the axis served as centres of rotation for freely rotating planes. The orientation where these planes had the minimal cross-sectional area of the 3D reconstructed structure was considered perpendicular to the longitudinal axis of the scanned ovipositor. To avoid merging of the segmented valves, we applied an erosion algorithm on the entire reconstruction before the determining the 
minimal cross-sections, and a dilation algorithm with the same parameters afterwards.

The second moment of area, a measure for structural bending resistance, was calculated for bending in the dorsi-ventral direction $\left(I_{\mathrm{x}}\right)$, which is the main direction of bending during normal probing. ${ }^{4}$ We analysed both the whole ovipositor (all three valves together) and its individual valves (formula provided in the SI). The data of the ventral valves were aligned using their clearly recognizable maxima in $I_{\mathrm{x}}$ and averaged to obtain a single curve representing both ventral valves. The mean ventral valve was aligned with the dorsal valve based on the location of the tip vertices. The histological and $\mu \mathrm{CT}$ datasets were corrected for size differences using predetermined scaling factors (see $S I$ ) and combined to form a full-length dataset.

\section{Bending stiffness}

For the bending tests, the sheaths surrounding the ovipositor were removed, and the ovipositors were cut close to their base at the abdomen. Subsequently, the ovipositors were kept submerged in physiological (Ringer's) insect buffer solution (see SI) at all times to prevent desiccation and to minimise changes in the material properties of the cuticle. To avoid damage to the ovipositor, we manipulated the samples only at the side opposite to the one intended for measurements. In total, we sampled data from 23 individuals. In 10 cases, the ovipositor valves were separated by carefully sliding them off each other. In most samples, the stiffness of their distal part was measured, but five ovipositors were measured at their proximal part. The dissected ovipositors or individual valves were clamped into a custommade clamp that kept the samples horizontal when mounted in the experimental setup (Fig. S6D).

\section{Experimental setup}

The set-up (Fig. S7A) was positioned on a vibration isolation table TS-150 (Table Stable, Zwilikon, Switzerland) to avoid external interference. It consisted of three parallel carbon steel razorblades (FA-10; Feather)—two on the bottom pointing up and one on the top pointing down. The bottom razorblades were glued in the centre of a square Petri dish, more than ten times the diameter of the ovipositor $(350.48 \mu \mathrm{m}$ or $357.90 \mu \mathrm{m}$ ) apart from each other. The horizontal position of the top blade was located exactly in between them. The Petri dish was fixed onto a Hexapod micromotion positioning system (Physik Instrumente, Karlsruhe, Germany) that allowed translations in all directions with sub-micrometre resolution. To prevent sample desiccation during measurements, the Petri dish was filled with the physiological 
(Ringer's) buffer solution for insects which was in this case degassed to avoid air bubbles forming on the sample surface.

The top razor was mounted onto a force transducer (FORT10g, dynamic range $10 \mathrm{~g}$, resolution $<0.01 \mathrm{mN}$; World Precision Instruments) fixed on a clamp stand and connected to a PC via a MP100WSW data acquisition system (Biopac Systems Inc., Goleta, CA, USA). The measurements were recorded with AcqKnowledge 3.7.0 software (Biopac System Inc., Goleta, CA, USA). The force transducer was calibrated by hanging a mass of $1655.3 \mathrm{mg}$ on the lever of the force transducer with the attached razorblade. Because the transducer behaved linearly within its dynamic range, a single weight was sufficient to calibrate it (Fig. S8).

Using the micromanipulator, the sample was placed perpendicular to the longitudinal axis of the razors and parallel to the bottom of the Petri dish. The Petri dish with the two razor blades was moved upwards in at least four steps (deflections) of $10 \mu \mathrm{m}$ with inter-step intervals of several seconds (variable in number). The bending was done at 4-6 locations along each tested sample, depending on its length and the location of the clamp (Chapter 4). The reaction forces on the top razorblade during bending were continuously recorded at a sampling frequency of $200 \mathrm{~Hz}$.

All three point bending tests were monitored with a camera (Basler piA1900-32g, Basler Vision Technologies, $2 \mathrm{MP}$ and $5 \mathrm{fps}$ ) mounted on a stereomicroscope and controlled with StreamPix 5 (NorPix Inc.) software. Light was provided by a cold light source isolated from the anti-vibrational table.

\section{Analysis of force data}

Analysis of the signal-to-noise (S/N) ratio with AcqKnowledge 3.7.3 software showed low values in highly flexible regions of the ovipositor such as the dorsal valves and the S-shaped region of the ventral valves (Fig. S7B). Low S/N recordings were filtered with an infinite impulse response (IIR) band-stop filter (frequency $50 \mathrm{~Hz}, Q=0.1$ ) to remove electrical noise and improve curve fitting in further analyses. The application of the IIR filter did not affect the accuracy of the fits (Fig. S7B).

Data was first manually divided into individual sections corresponding to individual deflections, such that the sections overlapped by 1 second. This allowed for accurate fitting of the step function to the data (see below). Based on the video recordings, we omitted all force recordings where the razorblades were not in contact with the sample throughout the deflection step. Second, to avoid the error in the computation of the force change between subsequent steps due to non-standardized duration of the deflection steps, the single step force data were fitted with a Heavi- 
side function adjusted for the exponential force relaxation:

$$
f(t)=H(t)\left(A\left(1-e^{-\lambda t}\right)+B\right)+C,
$$

where $t$ is time, $H(t)$ is the Heaviside step function, $A$ denotes the relaxation force value for each step, $\lambda$ is the exponential decay factor of the force relaxation, $B$ is the amplitude of the step response, and $C$ is the total offset of the curve. The time constant $(\tau=1 / \lambda)$ of the exponential decay was used to calculate the force increase within each deflection step as the value at $t=3 \tau$, which corresponds to $95.02 \%$ value of the steady state of the force response.

Data was corrected for stiffness of the experimental setup, which was determined by pressing two perpendicular razor blades against each other with $1 \mu \mathrm{m}$ steps and analysing the obtained data in the same manner as described above.

The forces and corrected deflections across consecutive steps were then fitted with an exponential decline function (Fig. S6C):

$$
F(d)=\alpha\left(1-\beta e^{-\gamma d}\right),
$$

where $F(d)$ is the force, $d$ is the deflection, $\alpha, \beta$, and $\gamma$, are coefficients describing the final amplitude, the initial offset, and the steepness of the curve, respectively. To get a good estimation of the natural condition we calculated the curvature in the samples and compared that with the observed curvature in probing animals (see SI). In general, only the first analysed deflection step fell within the naturally occurring curvatures (Fig. S9), so sample stiffness was estimated from the slope of the force-deflection curves at this point.

\section{Derived effective modulus of elasticity}

We obtained the bending stiffness (EI) of the ovipositor and the valves from the three point bend experiments using the Euler-Bernoulli beam theory, which states that:

$$
E I=\frac{F L^{3}}{48 d}
$$

were $d$ is the deflection, $F$ the bending force, and $L$ the distance between the outer two razor blades. Because the ovipositor mainly consists out of cuticle, a composite material consisting of polysaccharide filaments (chitin) embedded in a protein matrix with possible metal inclusions that varies spatially, 45,46 it was impossible to obtain a Young's modulus of the samples. But we could estimate an effective modulus of elasticity at each location along the ovipositor $\left(E_{\mathrm{eff}}(l)\right)$ by dividing the bending stiffness at each location by the second moment of area at the same lo- 


\section{II}

cations $\left(I_{\mathrm{x}}(l)\right.$; Fig. 3$)$. The $I_{\mathrm{x}}(l)$ values were obtained from histological and $\mu \mathrm{CT}$ data and were multiplied by the correction factor $\left(c_{\mathrm{f}}\right.$; see $\left.S I\right)$ to adjust them for size:

$$
E_{\mathrm{eff}}(l)=\frac{F(l) \cdot L^{3}}{48 d \cdot I_{\mathrm{x}}(l) \cdot c_{\mathrm{f}}}
$$

The locations of force measurements along the sample length were obtained from the videos of the three point bending experiments.

\section{Confocal laser scanning microscopy}

The material composition of the ovipositor was qualitatively assessed by visualizing autofluorescence in a confocal laser scanning microscope (ZeissLSM 700, Zeiss) with a $10 \times$ and $20 \times$ objective lenses (Zeiss Plan-Apochromat; numerical apertures 0.45 and 0.8 , respectively). We applied the method established by [47], which provides reliable information on the presence of resilin in insect cuticles. The ovipositors were put on a glass slide, embedded in glycerin, and covered with a cover glass. The sample was left under the microscope for half an hour to stabilize in the medium before scanning. Zeiss Efficient Navigation (ZEN) software (Carl Zeiss MicroImaging $\mathrm{GmbH}$ ) was used to record the autofluorescence and create maximum intensity overlay images. We used excitation lasers of 405, 488, 555, and $639 \mathrm{~nm}$ and recorded the Z-stacks of autofluorescence using a $420-480 \mathrm{~nm}$ band-pass filter and $\geq 490, \geq 560$, and $\geq 640 \mathrm{~nm}$ long-pass filters, respectively. Before every scan, the laser power and detector gain of each laser were manually adjusted in live view at the scanning speed that was also used for the acquisition of the final image. We first increased both parameters until observing pixel saturation. Afterwards, the two parameters were carefully reduced to obtain an image with minimal number of the saturated pixels, before taking the micrographs. In the final image, the fluorescence light emissions were assigned to blue, green, red (50\% intensity), and red (50\% intensity) channels, respectively, according to the colour code by [47].

\section{Acknowledgements}

We acknowledge the Paul Scherrer Institut, Villigen, Switzerland for provision of synchrotron radiation beam time at the TOMCAT beamline X02DA of the SLS and would like to thank Chirstian Schlepuetz for invaluable assistance. We thank the Naturalis Biodiversity Center (RMNH; Leiden, Netherlands) and collection manager Frederique Bakker for help and providing the specimen used for CT-scanning. We also acknowledge the National Institute of General Medical Sciences of the National Institutes of Health for their role in the development of the Seg3D software (grant number P41 GM103545-18) for segmentation purposes. We thank Henk 
Schipper and Frank van den Berg for their help with histological sections of the ovipositor; Karen Leon-Kloosterziel for technical support, animal care, and help with ovipositor scaling analysis; and the user committee of the Netherlands Organization for Scientific Research Division Applied and Engineering Sciences (NWO TTW) WASP project and the members of the research group for their useful discussions. This work was supported by NWO domain applied and engineering sciences (NWO TTW). 


\section{References}

[1] Соок JM \& RAsplus JY (2003). Mutualists with attitude: coevolving fig wasps and figs. Trends in Ecology and Evolution 18, 241-248.

[2] Vilhelmsen L, Isidoro N, Romani R, Basibuyuk HH, \& Quicke DLJ (2001). Host location and oviposition in a basal group of parasitic wasps: the subgenual organ, ovipositor apparatus and associated structures in the Orussidae (Hymenoptera, Insecta). Zoomorphology 121, 63-84.

[3] Pennacchio F \& Strand MR (2006). Evolution of developmental strategies in parasitic Hymenoptera. Annual Review of Entomology 51, 233-258.

[4] Cerkvenik U, Van de Straat B, Gussekloo SWS, \& van Leeuwen JL (2017). The mechanisms of ovipositor insertion and steering of a parasitic wasp. Proceedings of the National Academy of Sciences 114 (37), E7822-E7831.

[5] Elias LG, Teixeira SP, Kjellberg F, \& Santinelo Pereira RA (2012). Diversification in the use of resources by Idarnes species: bypassing functional constraints in the fig-fig wasp interaction. Biological Journal of the Linnean Society 106, 114-122.

[6] Quicke DLJ, Fitton M, \& Harris J (1995). Ovipositor steering mechanisms in braconid wasps. Journal of Hymenoptera Research 4, 110-120.

[7] Sмітн EL (1970). Evolutionary morphology of the external insect genitalia. 2. Hymenoptera. Annals of the Entomological Society of America 63, 1-27.

[8] Quicke DLJ, Fitton MG, Tunstead JR, Ingram SN, \& Gaitens PV (1994). Ovipositor structure and relationships within the Hymenoptera, with special reference to the Ichneumonoidea. Journal of Natural History 28, 635-682.

[9] SмIтн EL (1969). Evolutionary morphology of external insect genitalia. 1. Origin and relationships to other appendages. Annals of the Entomological Society of America 62, 1051-1079.

[10] VINCENT JFV \& KING MJ (1995). The mechanism of drilling by wood wasp ovipositors. Biomimetics 3, 187-201.

[11] Quicke DLJ (1991). Ovipositor mechanics of the braconine wasp genus Zaglyptogastra and the ichneumonid genus Pristomerus. Journal of Natural History 25, 971-977.

[12] Fergusson NDM (1988). A comparative study of the structures of phylogenetic importance of female genitalia of the Cynipoidea (Hymenoptera). Systematic Entomology 13, 13-30.

[13] SCHUDDER GGE (1961). The comparative morphology of the insect ovipositor. Transactions of the Royal Entomological Society of London 113, 2-40.

[14] Rousse P \& VAN Noort S (2015). Revision of the Afrotropical species of Pristomerus (Ichneumonidae: Cremastinae), with descriptions of 31 new species. European Journal of Taxonomy 1-129.

[15] El-Heneidy AH \& Quicke DL (1991). The Indo-Australian species of the braconine wasp genus Zaglyptogastra Ashmead. Journal of Natural History 25, 183-201.

[16] WeIs-Fogh T (1960). A rubber-like protein in insect cuticle. Journal of Experimental Biology 37, 889907.

[17] Burrows M, Shaw SR, \& Sutton GP (2008). Resilin and chitinous cuticle form a composite structure for energy storage in jumping by froghopper insects. BMC Biology 6, 41.

[18] Rothschild M \& Schlein J (1975). The jumping mechanism of Xenopsylla cheops I. Exoskeletal structures and musculature. Philosophical Transactions of the Royal Society of London. Series B, Biological Sciences 271, 457-490.

[19] HaAs F, Gorb S, \& Blickhan R (2000). The function of resilin in beetle wings. Proceedings of the Royal Society B: Biological Sciences 267, 1375-1381.

[20] Appel E, Heepe L, Lin CP, \& GoRb SN (2015). Ultrastructure of dragonfly wing veins: Composite structure of fibrous material supplemented by resilin. Journal of Anatomy 227, 561-582.

[21] Peisker H, Michels J, \& Gorb SN (2013). Evidence for a material gradient in the adhesive tarsal setae of the ladybird beetle Coccinella septempunctata. Nature Communications 4, 1607-1608. 
[22] Bennet-Clark H (1997). Tymbal mechanics and the control of song frequency in the cicada Cyclochila australasiae. The Journal of experimental biology 200, 1681-1694.

[23] Choumet V, Attout T, Chartier L, Khun H, Sautereau J, Robbe-Vincent A, Brey P, Huerre M, \& BAIN O (2012). Visualizing non infectious and infectious Anopheles gambiae blood feedings in naive and saliva-immunized mice. PloS one 7 , e50464.

[24] Leopold RA, Freeman TP, Buckner JS, \& Nelson DR (2003). Mouthpart morphology and stylet penetration of host plants by the glassy-winged sharpshooter, Homalodisca coagulata, (Homoptera: Cicadellidae). Arthropod Structure \& Development 32, 189-199.

[25] Kundanati L \& Gundiah N (2014). Biomechanics of substrate boring by fig wasps. Journal of Experimental Biology 217, 1946-1954.

[26] Matsumura Y, Kovalev AE, \& Gorb SN (2017). Penetration mechanics of a beetle intromittent organ with bending stiffness gradient and a soft tip. Science Advances 3, eaao5469.

[27] Fulton BB (1932). Notes on Habrocytus cerealellae, parasite of the Angoumois grain moth. Annals of the Entomological Society of America 26, 536-553.

[28] Whiting AR (1967). The biology of the parasitic wasp Mormoniella vitripennis (= Nasonia Brevicornis) (Walker). The Quarterly Review of Biology 42, 334-406.

[29] Austin AD \& Browning TO (1981). A mechanism for movement of eggs along insect ovipositors. International Journal of Insect Morphology and Embryology 10, 93-108.

[30] Austin AD (1983). Morphology and mechanics of the ovipositor system of Ceratobaeus Ashmead (Hymenoptera: Scelionidae) and related genera. International Journal of Insect Morphology and Embryology 12, 139-155.

[31] BRonNer R (1985). Anatomy of the ovipositor and oviposition behavior of the gall wasp Diplolepis rosae (Hymenoptera: Cynipidae). Can. Ent. 117, 849-858.

[32] Leyva JL, Browning HW, \& Gilstrap FE (1991). Effect of host fruit species, size, and color on parasitization of Anastrepha ludens (Diptera: Tephritidae) by Diachasmimorpha longicaudata (Hymenoptera: Braconidae). Environmental Entomology 20, 1469-1474.

[33] Fidelibus MW, Teixeira AA, \& Davies FS (2002). Mechanical properties of orange peel and fruit treated pre-harvest with gibberellic acid. Transactions of the American Society of Agricultural Engineers 45, 1057-1062.

[34] Wharton R, Ward L, \& Miкo I (2012). New neotropical species of Opiinae (Hymenoptera, Braconidae) reared from fruit-infesting and leafmining Tephritidae (Diptera) with comments on the Diachasmimorpha mexicana species group and the genera Lorenzopius and Tubiformopius. ZooKeys 243, 27-82.

[35] Van Achterberg C (1999). The Palaearctic species of the genus Diachasmimorpha Viereck (Hymenoptera: Braconidae: Opiinae). Zoologische Mededelingen 73, 1-10.

[36] Gordon RM \& LumSDen WHR (1939). A study of the behaviour of the mouth-parts of mosquitoes when taking up blood from living tissue together with some observations on the ingestion of microfiliarie. Annals of tropical medicine and parasitology 33, 259-278.

[37] Pollard DG (1973). Plant penetration by feeding aphids (Hemiptera, Aphidoidea): a review. Bulletin of Entomological Research 62, 631.

[38] Pollard DG (1970). The mechanism of stylet movement in Psylla mali Schmidberger (Homoptera: Psyllidae). Zoological Journal of the Linnean Society 49, 295-307.

[39] Ko SY, Davies BL, \& Rodriguez y Baena F (2010). Two-dimensional needle steering with a "programmable bevel" inspired by nature: Modeling preliminaries. Intelligent Robots and Systems (IROS), 2010 IEEE/RSJ International Conference on 2319-2324.

[40] Scali M, Pusch T, Breedveld P, \& Dodou D (2017). Ovipositor-inspired steerable needle: design and preliminary experimental evaluation. Bioinspiration \& Biomimetics 13, 016006.

[41] Ko SY, Frasson L, \& Rodriguez y Baena F (2011). Closed-loop planar motion control of a steerable probe with a 'programmable bevel' inspired by nature. IEEE Transactions on Robotics 27, 970-983. 
[42] Marone F \& Stampanoni M (2012). Regridding reconstruction algorithm for real-time tomographic imaging. Journal of Synchrotron Radiation 19, 1029-1037.

[43] Paganin D, Mayo S, Gureyev T, Miller P, Wilkins S, Scientific C, \& Science M (2002). Simultaneous phase and amplitude extraction from a single defocused image of a homogeneous object. Journal of Microscopy 206, 33-40.

[44] CIBC (2016), Seg3D: Volumetric Image Segmentation and Visualization. Scientific Computing and Imaging Institute (SCI), Download from: http:/ / www.seg3d.org.

[45] VINCENT JFV (2002). Arthropod cuticle: a natural composite shell system. Composites Part A: Applied Science and Manufacturing 33, 1311-1315.

[46] Vincent JFV \& Wegst UGK (2004). Design and mechanical properties of insect cuticle. Arthropod Structure \& Development 33, 187-199.

[47] Michels J \& Gorb SN (2012). Detailed three-dimensional visualization of resilin in the exoskeleton of arthropods using confocal laser scanning microscopy. Journal of Microscopy 245, 1-16.

[48] Gere JM (2008). Mechanics of Materials. Belmont, Brooks/Cole-Thomson Learning, 6th edn.

[49] Barbosa P, Berry DL, \& Kary CSK (2015). Insect Histology: Practical Laboratory Techniques. Insect Histology: Practical Laboratory Techniques 1-348. 


\section{Supporting information}

\section{SI Figures}

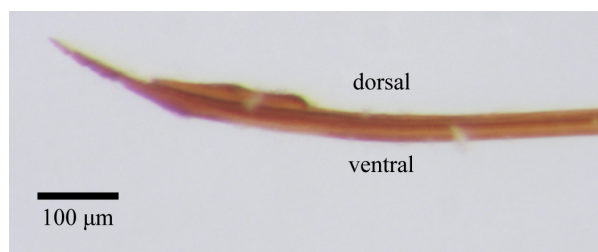

Fig. S1. The tip of an isolated ovipositor with protracted ventral valves curves outside a solid substrate. Upon protraction of the ventral valves, the ovipositor tip curves dorsally, which indicates to an inherent curving mechanism. The S-shaped region of the ovipositor is straightened, presumably due to the stiff, widened region of the dorsal valve tip (bulge).
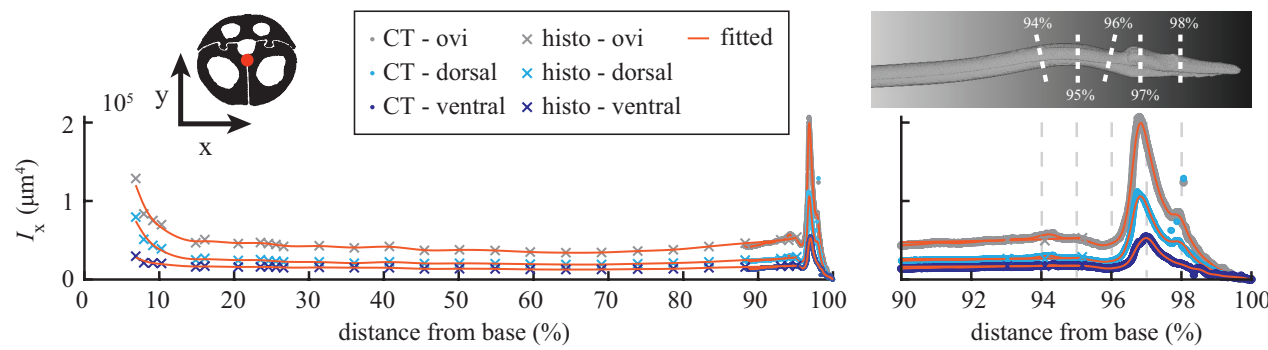

Fig. S2. Contribution to the ovipositor second moment by individual valves. $I_{\mathrm{x}}$ values for the valves are higher than in Fig. 3A because we used the centroid of the whole ovipositor (red dot in the schematics) in all calculations. The dorsal valve contributes more to the ovipositor second moment of area than each individual ventral valve, because compared to the ventral valves, a greater part of the dorsal valve is located further away from the ovipositor centroid 


\section{II}
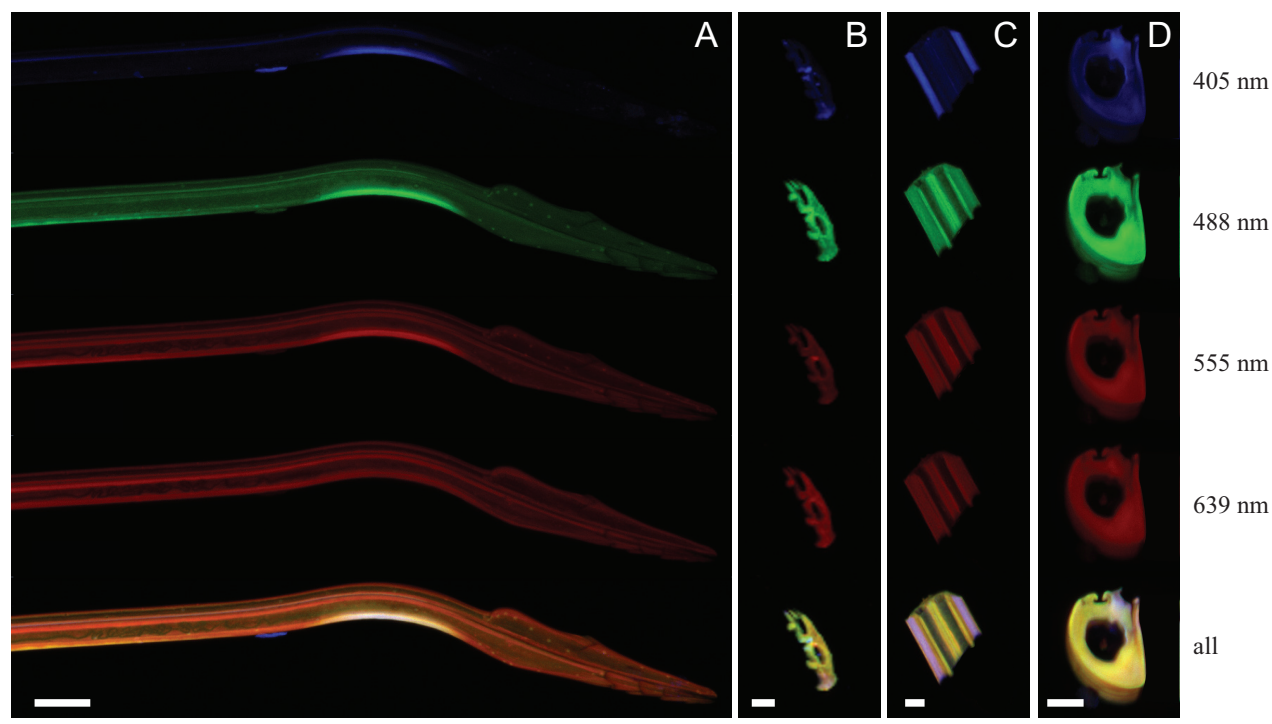

Fig. S3. Autofluorescences (split in different channels) recorded from the ovipositor. Autofluorescence of materials excited with different wavelengths shows gradients in material composition in the distal part of the ovipositor. Excitation wavelengths indicated in the figure on the right. (A) Distal part of the ovipositor. (B-D) Sections from the distal region of the ovipositor, but proximal to its S-shaped region. (B) Dorsal valve cross-section. (C) Dorsal valve inner side. (D) Ventral valve cross section. Scale bars: $50 \mu \mathrm{m}(\mathrm{A}), 10 \mu \mathrm{m}(\mathrm{B}-\mathrm{D})$. 


\section{SI Tables}

Table S1: Mean second moment of area of regions of fitted data based on CT-scans only. For centroids see Fig. 3. The shaft, S-region and tip are for each structure significantly different (Mann-Whitney U test, $P<0.05)$. The bulge is defined as the part of the tip where $I_{\mathrm{X}}$ is larger than the mean $I_{\mathrm{X}}$ of the shaft.

\begin{tabular}{|c|c|c|c|c|}
\hline & \multicolumn{4}{|c|}{ Second moment of area $\left(I_{x}, 1 \times 10^{4} \mathrm{~m}^{4}\right)$} \\
\hline & $\begin{aligned} & \text { Shaft } \\
< & 93 \% \text { OL }\end{aligned}$ & $\begin{array}{c}\text { S-region } \\
93-96 \%\end{array}$ & $\begin{array}{l}\text { Tip-region } \\
>96 \% \text { OL }\end{array}$ & Bulge \\
\hline $\begin{array}{c}\text { Dorsal } \\
\text { valve }\end{array}$ & $\begin{array}{c}0.25 \pm 0.02 \\
\max =0.27 \\
(n=1655)\end{array}$ & $\begin{array}{c}0.30 \pm 0.06 \\
\max =0.39 \\
(n=999)\end{array}$ & $\begin{array}{c}1.27 \pm 1.05 \\
\max =3.06 \\
(n=1227)\end{array}$ & $\begin{array}{c}96.29-99.41 \% \text { OL } \\
\text { mean }=1.60 \pm 0.97 \\
(n=941)\end{array}$ \\
\hline $\begin{array}{l}\text { Ventral } \\
\text { valve }\end{array}$ & $\begin{array}{c}0.94 \pm 0.10 \\
\max =1.13 \\
(n=1555)\end{array}$ & $\begin{array}{c}1.06 \pm 0.10 \\
\max =1.16 \\
(n=1001)\end{array}$ & $\begin{array}{c}0.55 \pm 0.45 \\
\max =1.61 \\
(n=1229)\end{array}$ & $\begin{array}{c}96.14-97.21 \% \text { OL } \\
\text { mean }=1.30 \pm 0.22 \\
(n=347)\end{array}$ \\
\hline $\begin{array}{c}\text { Whole } \\
\text { ovipositor }\end{array}$ & $\begin{array}{c}4.51 \pm 0.28 \\
\max =5.04 \\
(n=1665)\end{array}$ & $\begin{array}{c}5.13 \pm 0.39 \\
\max =5.74 \\
(n=1018)\end{array}$ & $\begin{array}{c}7.31 \pm 5.78 \\
\max =19.93 \\
(n=1208)\end{array}$ & $\begin{array}{c}95.99-98.34 \% \text { OL } \\
\text { mean }=10.21 \pm 4.82 \\
(n=769)\end{array}$ \\
\hline
\end{tabular}

Table S2: Measured bending stiffness and calculated effective modulus of elasticity of the shaft and S-region of ovipositors, dorsal and ventral valves. Diff. indicates the significance level of the difference between the shaft and S-region for the specific parameter obtained with a Mann-Whitney U test. When comparing values within each column, only the $E_{\text {eff }}$ of the shaft of the ventral valve and the whole ovipositor do not significantly differ (Mann-Whitney U test, $P=$ N.S.).

\begin{tabular}{|c|c|c|c|c|c|c|c|}
\hline & \multicolumn{3}{|c|}{$\begin{array}{c}\text { Bending stiffness } \\
\left(E_{\text {eff }} I_{x}, 1 \times 10^{-11} \mathrm{~N} \mathrm{~m}^{2}\right)\end{array}$} & & \multicolumn{3}{|c|}{$\begin{array}{c}\text { Effective modulus of elasticity } \\
\left(E_{\text {eff }}, \mathrm{GPa}\right)\end{array}$} \\
\hline & $\begin{aligned} & \text { Shaft } \\
&<93 \% \text { OL }\end{aligned}$ & $\begin{array}{l}\text { S-region } \\
93-96 \%\end{array}$ & Diff. & & $\begin{aligned} & \text { Shaft } \\
< & 93 \% \text { OL }\end{aligned}$ & $\begin{array}{l}\text { S-region } \\
93-96 \%\end{array}$ & Diff. \\
\hline $\begin{array}{l}\text { Dorsal } \\
\text { valve }\end{array}$ & $\begin{array}{c}0.90 \pm 0.33 \\
(n=30)\end{array}$ & $\begin{array}{c}0.88 \pm 0.52 \\
(n=30)\end{array}$ & $P=$ N.S. & & $\begin{array}{c}5.95 \pm 2.53 \\
(n=30)\end{array}$ & $\begin{array}{c}3.70 \pm 2.51 \\
(n=10)\end{array}$ & $P<0.01$ \\
\hline $\begin{array}{l}\text { Ventral } \\
\text { valve }\end{array}$ & $\begin{array}{l}4.40 \pm 1.85 \\
(n=26)\end{array}$ & $\begin{array}{l}1.57 \pm 0.85 \\
(n=10)\end{array}$ & $P<0.001$ & $\dot{n}$ & $\begin{array}{l}4.81 \pm 2.56 \\
(n=26)\end{array}$ & $\begin{array}{l}1.46 \pm 0.74 \\
(n=10)\end{array}$ & 0.001 \\
\hline $\begin{array}{l}\text { Whole } \\
\text { ovipositor }\end{array}$ & $\begin{array}{l}15.72 \pm 5.37 \\
\quad(n=42)\end{array}$ & $\begin{array}{l}5.12 \pm 1.09 \\
\quad(n=11)\end{array}$ & $P<0.001$ & 11 & $\begin{array}{l}3.60 \pm 1.27 \\
\quad(n=42)\end{array}$ & $\begin{array}{l}0.92 \pm 0.35 \\
(n=11)\end{array}$ & 0.001 \\
\hline
\end{tabular}




\section{SI Finite element analysis}

The effect of clamping and pre-bent beam shape was investigated with FE simulations using Abaqus/CAE 2017 (Simulia, Dassault Systemes). Because this question does not require a complete description of geometrical and material valve properties, we simulated solid cylindrical beams. To reduce the computational time, we took advantage of the lateral symmetry of the bending simulation and longitudinally halved the model and assumed lateral symmetry in the cut plane. The beam was therefore modelled as a half cylinder (length $5 \mathrm{~mm}$, radius $7.5 \mu \mathrm{m}$ ). For the effect of clamping, we used homogeneous material properties with a Poisson's ratio $(v)$ of 0.3 , while the Young's modulus $(E)$ was varied and set at $0.1,1$ and $10 \mathrm{GPa}$. Both parameters are within the range of their estimated values for insect cuticle. $^{45}$

Bending was simulated such that it closely resembled the experimental conditions; one end of the beam was clamped and the razorblades were modelled as rectangular pins with rounded corners (width and height $5 \mu \mathrm{m}$, depth $8 \mu \mathrm{m}$, Fig. S4A). The pins were positioned perpendicular to the beam and parallel to each other-the bottom two pins were $350 \mu \mathrm{m}$ apart and the top one was in their middle. The middle pin was located on top of the beam, the outer two pins on its bottom side. One end of the beam and the top pin were fixed in all three dimensions, while the bottom two pins were only allowed to move upwards and rotate in the vertical plane that was cutting the beam in half. The pins were modelled as non-deformable rigid bodies, thus resembling steel razorblades of much higher stiffness than the ovipositor. Additionally, we assumed frictionless contact between the beam and the pins and only considered the normal forces. As in the experiment, the bottom two pins were pushed towards the top one in three $10 \mu \mathrm{m}$ steps. The vertical reaction forces (i.e. those in the bending direction) in the pins and at the fixed end of the beam were extracted for each deflection.

To determine the effect of the S-shape near the tip of the ovipositor, we created a cylinder with a similar S-shape located $4 \mathrm{~mm}$ away from the clamped end (Fig. S4B). The ovipositor geometry was estimated from the micrographs $(n=4)$. The Sshaped FEM beam consisted of a proximal bend in dorsal direction of $10^{\circ}$ from its longitudinal axis, followed by a $20^{\circ}$ bend in the ventral direction, and a $10^{\circ}$ bend in dorsal direction again. The bends were spaced $100 \mu \mathrm{m}$ apart and were rounded $(r=150 \mu \mathrm{m})$ to avoid structural discontinuities and the associated stress accumulation. The beam was assigned an $E=10 \mathrm{GPa}$ and $v=0.3$. 

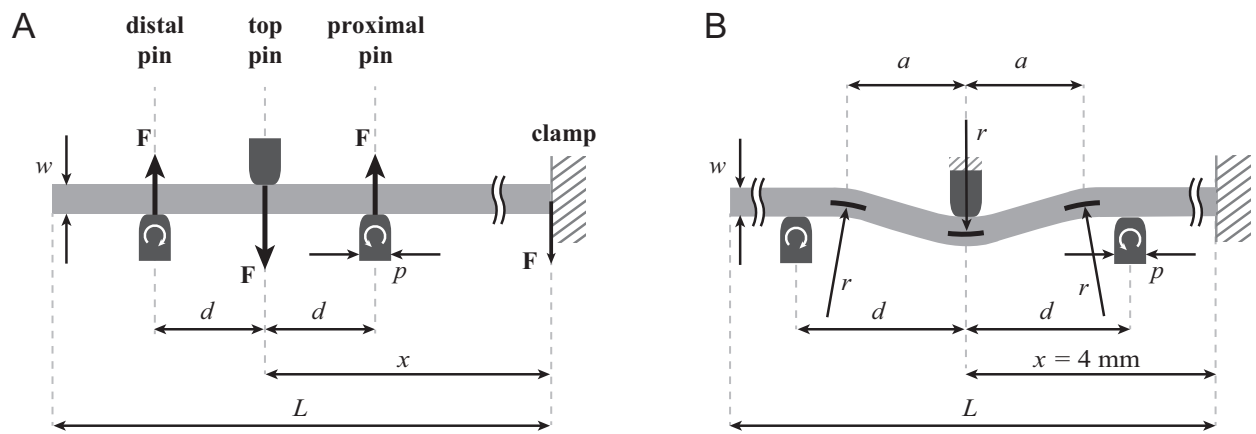

Fig. S4. FEM simulations of three point bending tests on cantilever beams. (A) Schematics of the FEM model. The cantilever beam of width $w=15 \mu \mathrm{m}$ and length $L=5 \mathrm{~mm}$ was bent between three rectangular pins with rounded corners of width $p=5 \mu \mathrm{m}$. The distance between the bottom two pins was $350 \mu \mathrm{m}(=2 d)$ and the top pin was positioned in their middle. The pins were modelled as rigid bodies. The top pin was fixed in all directions and the bottom two pins were allowed to rotate in plane indicated in the schematics (white arrows). Bending was done at four different distances from the clamp $(x)$ and the vertical reaction forces $(F)$ in the pins and at the fixed end of the cantilever were computed. (B) Schematics of model used to determine the effect of the bent beam geometry. The symbols are same as in (A), except for $a=100 \mu \mathrm{m}$ and $r=150 \mu \mathrm{m}$ which denote the length of the curved region and maximal local radius of curvature in the curve (short, black lines), respectively.

\section{SI Mesh convergence}

To determine the effect of the mesh size on the model output, we performed multiple simulations where the mesh size was varied, while keeping all other parameters constant. The mesh grid size was varied between $0.5 \mu \mathrm{m}$ and $2 \mu \mathrm{m}$ in steps of $0.5 \mu \mathrm{m}$ both along the length of the beam and its perimeter. In these simulations, the bending location was kept at $4 \mathrm{~mm}$ from the clamp, the stiffness of the beam $(E)$ was $10 \mathrm{GPa}$, and its Poisson's ratio $(v)$ was 0.3 . The mesh size has little effect on the simulation output and the mesh grid size of $1 \mu \mathrm{m}$ was used in subsequent simulations (Fig. S5A).

\section{SI Effects of clamping on the force recordings}

The clamp effect was estimated by bending the beam at several distances from its fixed end, namely $1 \mathrm{~mm}, 2 \mathrm{~mm}, 3 \mathrm{~mm}$ and $4 \mathrm{~mm}$ from the clamp. Clamping has little effect on soft beams $(E \leq 1 \mathrm{GPa})$, whereas at higher beam stiffnesses $(E=10 \mathrm{GPa}$ and presumably above), the recorded forces strongly increase with a decreased distance to the clamp (Fig. S5B). If the force in the top pin differed for more than $5 \%$ compared to the measurements done at the location farthest away from the clamp, we considered the force measurements inaccurate. To obtain the distance from the clamped end at which this threshold is reached, we interpolated the data for $10 \mathrm{GPa}$ beam using a cubic spline. The threshold distance was $1.87 \mathrm{~mm}$ (Fig. S5B). We therefore omitted all data points that were done at distances $\leq 1.87 \mathrm{~mm}$ from the clamp from our experimental dataset. 
A

A

II

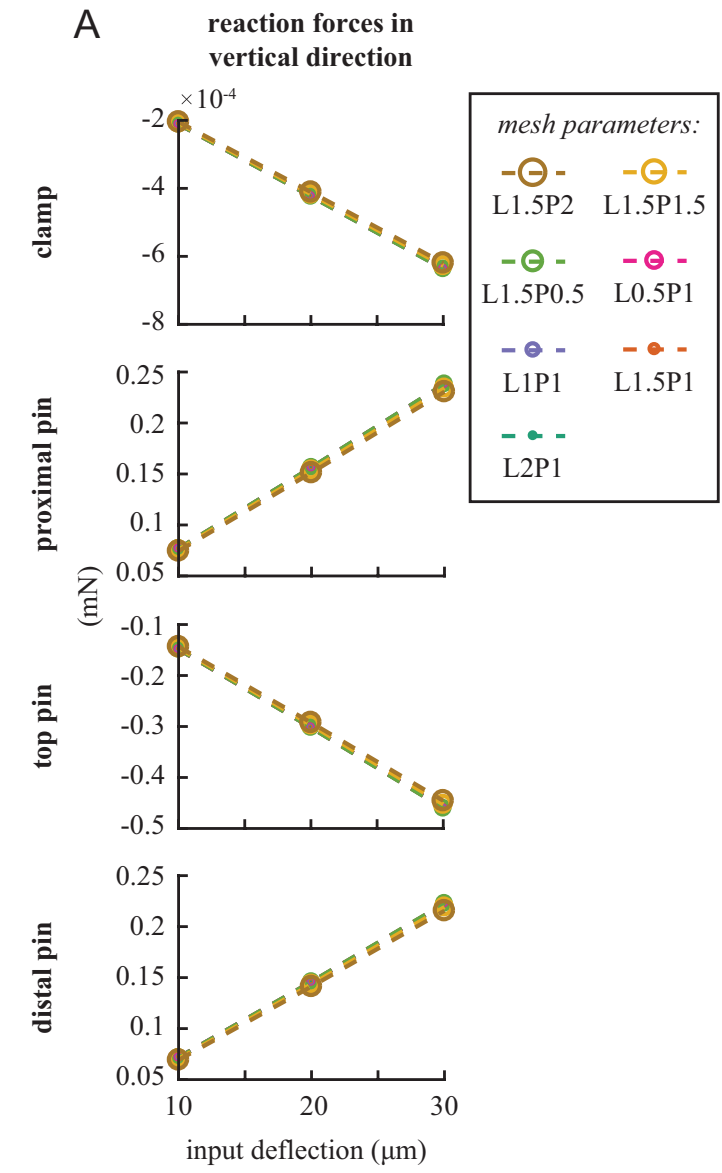

B

reaction forces at maximal input deflection

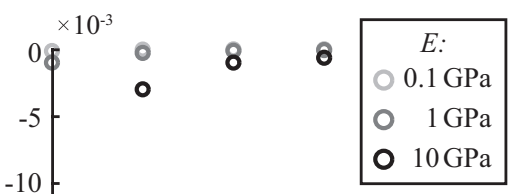

Fig. S5. Reaction forces in the vertical direction during deflections at the clamped end of the beam and the three pins. (A) Reaction forces during all three input deflections. Several simulations at $x=4 \mathrm{~mm}$ were performed while varying the mesh density both along the length and the perimeter of the beam. The seed sizes are indicated in the legend; for example, L2P1 indicates $2 \mu \mathrm{m}$ grid along the length of the beam and $1 \mu \mathrm{m}$ mesh along its perimeter. The mesh density has a negligible effect on the reaction forces. Final simulations were therefore performed with the mesh grid size of $1 \mu \mathrm{m}$. (B) Vertical force during maximal deflection $(30 \mu \mathrm{m})$. Shades of grey correspond to different beam stiffnesses $(E$; darker colours indicate stiffer beams); the light blue circle in the graph with the results for the top pin indicates the bent-beam $(E=10 \mathrm{GPa})$ simulation. Clamping has little effect when the Young's modulus $(E)$ of the beam is smaller or equal to $1 \mathrm{GPa}$. In stiffer cantilever beams $(E=10 \mathrm{GPa})$, the recorded forces increase with the vicinity of the clamp. The data for the top pin was interpolated using a cubic spline (black line) to calculate the reliability threshold for the force measurements. The threshold was set to the force difference of $5 \%$ or more compared to the output at $4 \mathrm{~mm}$ (black, vertical, dashed line).

\section{SI Effect of S-shaped morphology}

The force in the bent beam $(-0.43 \mathrm{mN})$ was only $7 \%$ smaller than for a straight beam $(-0.46 \mathrm{mN}, \mathrm{S} 5 \mathrm{~B})$. 


\section{SI Second moment of area calculation $\left(I_{\mathrm{x}}\right)$}

For the bending axes of the relevant structures needed for calculating $I_{x}$, we used the axes formed by their respective centroids in each slice (Fig. 2C; red circle is the ovipositor axis, red stars the valve axes).

The $I_{\mathrm{x}}$ was approximated using:

$$
I_{\mathrm{x}}=\sum_{i=1}^{n} y_{i}^{2} A_{p i x}
$$

where $y$ is the distance of the pixels from the horizontal axis going through the centroid of the cross-section, $A_{\text {pix }}$ is the area represented by an individual pixel, and $n$ is the number of pixels constituting the relevant cross-sectional area (complete ovipositor or individual valve).

\section{SI Ovipositor scaling analysis}

To correct for size differences between ovipositors, we calculated two scaling factors using a randomly chosen set of animals $(n=33)$ from our breeding colony. The ovipositors were dissected in phosphate buffer (see below) to prevent desiccation and photographed using an Olympus DP50 camera (5 MP; Olympus, Japan) mounted on the Zeiss Stemi SV 11 stereomicroscope with the $1.6 \times$ objective (Zeiss, Germany). The images were stored as tiff files and analysed with analySIS FIVE software (Olympus, Japan). The tip length was measured from the distal point of the dorsal valve to the apex of its enlargement. The ovipositor width was measured two tip lengths from its distal vertex. We correlated both these measures with the overall length of the ovipositor (Fig. S6A,B). The slope of the major axis regression lines were used to calculate the sample lengths based on their tip length. This allowed us to express the measurement locations (distances along each sample) as percentages of the sample length (Fig. 3).

To compute the correction factor for calculating the second moment of area of differently sized samples, we assumed isometric scaling of the ovipositors and that they can be represented by a simplified geometry. Specifically, the ovipositor was considered as a hollow cylinder, the dorsal valve as a hollow half-cylinder, and the ventral valves as hollow quarter-cylinders. Formulas for the calculating the second moment of area of these shapes differ only in their constants which cancel each other when deriving the correction factor, so we present here only the derivation for hollow quarter-cylinders. 


\section{II}

For a hollow quarter cylinder, the second moment of area with respect to its centroid $\left(I_{\mathrm{x}}\right)$ is calculated with [48]):

$$
I_{\mathrm{x}}=\left(\frac{\pi}{16}-\frac{4}{9 \pi}\right)\left(r_{1}^{4}-r_{2}^{4}\right)
$$

where $r_{1}$ and $r_{2}$ are respectively the outer and the inner radius of the full-cylinder cross-section (Fig. S6C). A correction factor $\left(c_{f}\right)$ is obtained by taking the ratio of $I_{\mathrm{x}}$ of two differently sized hollow cylinders:

$$
\frac{I_{\mathrm{x}, i}}{I_{\mathrm{x}, j}}=\frac{\left(\frac{\pi}{16}-\frac{4}{9 \pi}\right)\left(r_{1, i}^{4}-r_{2, i}^{4}\right)}{\left(\frac{\pi}{16}-\frac{4}{9 \pi}\right)\left(r_{1, j}^{4}-r_{2, j}^{4}\right)}=\frac{r_{1, i}^{4}-r_{2, i}^{4}}{r_{1, j}^{4}-r_{2, j}^{4}}=c_{\mathrm{f}} .
$$

For the ventral valves $r_{1}=2 r_{2}$, which was estimated from the segmented histological sections. However, obtaining these values for all samples was not feasible. Instead, we used the relationships between ovipositor width and its length and between the dorsal valve tip length and the ovipositor length (Fig. S6A,B) to express the correction factor in terms of the sample dorsal valve tip length $\left(l_{\mathrm{t}}\right)$.

The ovipositor width $\left(2 r_{1}\right)$ vs length $(L)$ relationship can be written as:

$$
r_{1}=\frac{s_{\mathrm{W}}}{2} L,
$$

where $s_{\mathrm{W}}$ is the width scaling factor. Taking into account the relationship between the radii, we can write a similar equation for the inner radius:

$$
r_{2}=\frac{s_{\mathrm{W}}}{4} L,
$$

Additionally, the dorsal tip length $\left(l_{\mathrm{t}}\right)$ varies with the ovipositor length with tip scaling factor $s_{\mathrm{t}}$ :

$$
l_{\mathrm{t}}=s_{1} L \Rightarrow L=\frac{l_{\mathrm{t}}}{s_{1}} .
$$

Equation S3 can be rewritten by expressing the radii as their respective width scaling factors and sample lengths (i.e. combining with equations S4 and S5) and substituting the sample lengths using equation S6: 


$$
\begin{gathered}
c_{\mathrm{f}}=\frac{r_{1, i}^{4}-r_{2, i}^{4}}{r_{1, j}^{4}-r_{2, j}^{4}}=\frac{\left(\frac{s_{\mathrm{w}}}{2} L_{i}\right)^{4}-\left(\frac{s_{\mathrm{w}}}{4} L_{i}\right)^{4}}{\left(\frac{s_{\mathrm{w}}}{2} L_{j}\right)^{4}-\left(\frac{s_{\mathrm{w}}}{4} L_{j}\right)^{4}}=\frac{\left(\frac{s_{\mathrm{w}}}{2}\right)^{4}\left(L_{i}^{4}-\left(\frac{L_{i}}{2}\right)^{4}\right)}{\left(\frac{s_{\mathrm{w}}}{2}\right)^{4}\left(L_{j}^{4}-\left(\frac{L_{j}}{2}\right)^{4}\right)}= \\
=\frac{\left(\frac{l_{\mathrm{t}, i}}{s_{1}}\right)^{4}-\left(\frac{l_{\mathrm{t}, i}}{2 s_{1}}\right)^{4}}{\left(\frac{l_{\mathrm{t}, j}}{s_{1}}\right)^{4}-\left(\frac{l_{\mathrm{t}, j}}{2 s_{1}}\right)^{4}}=\frac{\left(\mathrm{s}_{1}\right)^{4}\left(l_{\mathrm{t}, i}^{4}-\left(\frac{L_{\mathrm{t}, i}}{2}\right)^{4}\right)}{\left(\frac{1}{s_{1}}\right)^{4}\left(l_{\mathrm{t}, j}^{4}-\left(\frac{L_{\mathrm{t}, j}}{2}\right)^{4}\right)}=\frac{l_{\mathrm{t}, i}^{4}-\left(\frac{L_{\mathrm{t}, i}}{2}\right)^{4}}{l_{\mathrm{t}, j}^{4}-\left(\frac{L_{\mathrm{t}, j}}{2}\right)^{4}},
\end{gathered}
$$

Correction factors were calculated for each sample. Depending on the location of the measurement, the $c_{\mathrm{f}}$ was either computed with respect to the histological or $\mu \mathrm{CT}$ data set. The second moment of area obtained from the $\mu \mathrm{CT}$ scans were multiplied with the correction factors when calculating the second moments of area of the samples (Fig. 3A) and their effective moduli of elasticity (Fig. 3C).

A
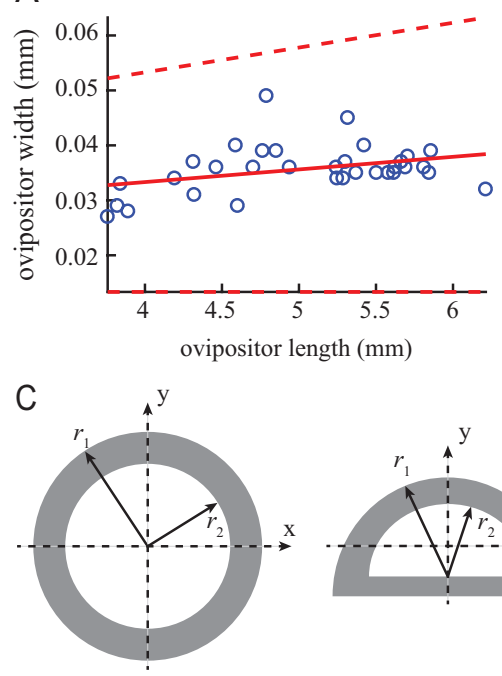

$\mathrm{B}$

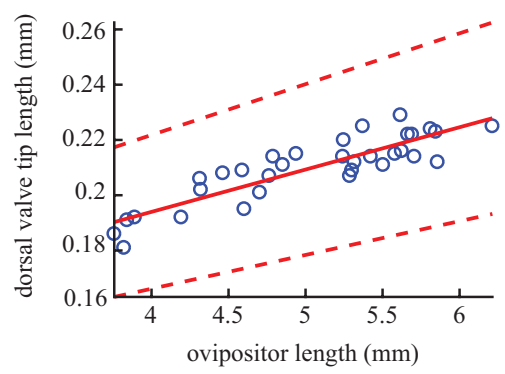

$\mathrm{D}$

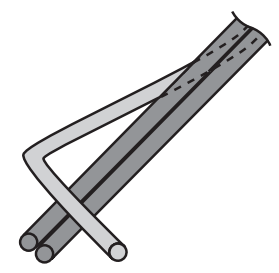

Fig. S6. Length of the morphologically distinct tip and the width of the ovipositor scale with its length. Data were obtained from 33 animals. (A) The ovipositor diameter increases with ovipositor length. (B) The length of the dorsal valve tip increases with ovipositor length. (C) Simplified crosssectional geometry used to determine the scaling factors. The cross-section of the ovipositor was modelled as a hollow cylinder, the dorsal valve that of a hollow half cylinder, and the ventral valve that of a hollow quarter cylinder. The lumina of the valves were assumed to have the same shape as the valves themselves. The radii of the outer shape and the lumina are denoted with $r_{1}$ and $r_{2}$, respectively. Intersection of dashed lines indicate the origin of the coordinate system used to calculate the second moment of area of the depicted structure. (D) Schematics of the holder made out of three insect pins used to clamp the samples during three point bending tests. Two straight pins were glued together. The third pin was bent in an L-shape; its straight end was glued to the other two pins, whereas its L-shaped end was twisted around the straight pins. The samples were fixed between the straight pins and the L-shaped pin. 


\section{SI Phosphate (PBS) buffer}

The phosphate buffer had a $\mathrm{pH}$ of 7.5 and was obtained by dissolving sodium chloride $(\mathrm{NaCl} ; 136.9 \mathrm{mM})$, potassium chloride $(\mathrm{KCl} ; 2.7 \mathrm{mM})$, monopotassium phosphate $\left(\mathrm{KH}_{2} \mathrm{PO}_{4} ; 1.5 \mathrm{mM}\right)$, and sodium hydrogen phosphate $\left(\mathrm{Na}_{2} \mathrm{HPO}_{4} \times 2 \mathrm{H}_{2} \mathrm{O}\right.$; $8.1 \mathrm{mM})$ in deionized water.

\section{SI Three point bending experimental set-up and analysis}
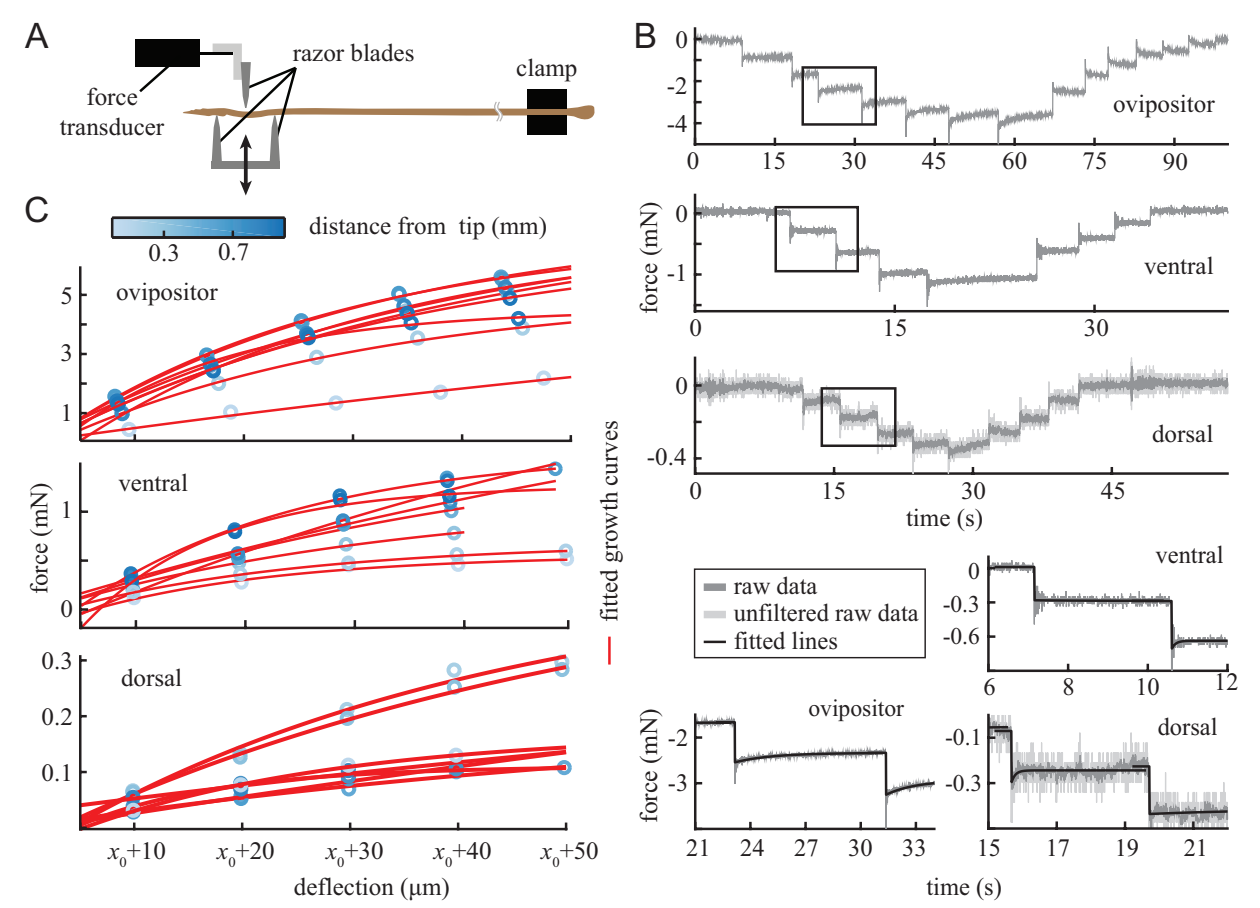

Fig. S7. Three point bending experiments along the ovipositor, ventral valve, and dorsal valve. (A) Schematics of the experimental setup. Samples were clamped at one end and positioned between three razorblades. Bending was achieved by pushing the bottom two razorblades towards the top one, which was connected to a force transducer. (B) Example data of force recordings for the entire ovipositor (all three elements together) and individual ventral and dorsal valves. Data used in the analysis are shown in black. These recordings contained electrical noise (i.e. light grey line) and were therefore filtered with a band stop IIR filter $(50 \mathrm{~Hz}, \mathrm{Q}=0.1)$ which reduced the noise before subsequent analysis. Increase in absolute value of the force during bending was calculated from curves fitted to individual deflections steps (bottom three graphs). (C) Example data of force $v$ s deflection for all three types of samples. We did not control for the distance between the sample and the top razor at the start of the bending. Therefore, the measurements differ in the amplitude of the initial deflection step $\left(x_{0}\right)$. The colour intensity of the blue symbols denotes location of bending along the sample length-darkness increases with distance from the tip. The data were fitted with an exponential decay function (increasing form) growth curve (red lines). The slope of the curve at the first recorded point was used to calculate the sample $E_{\text {eff }} I_{x}$. 


\section{SI Physiological (Ringer's) solution for insects}

The solution was prepared according to Barbosa et al., ${ }^{49}$ who recommend it as a normal salt solution for insect tissues. The units are expressed as parts (pt.) which refers to grams or millilitres, depending on whether the substance is a solid or a liquid. We mixed $0.8 \mathrm{pt}$. of sodium chloride $(\mathrm{NaCl}), 0.02 \mathrm{pt}$. of calcium chloride $\left(\mathrm{CaCl}_{2}\right), 0.02 \mathrm{pt}$. of potassium chloride $(\mathrm{KCl}), 0.02 \mathrm{pt}$. of sodium bicarbonate $\left(\mathrm{NaHCO}_{3}\right)$ with distilled water (100 pt.).

\section{SI Force transducer characteristics}
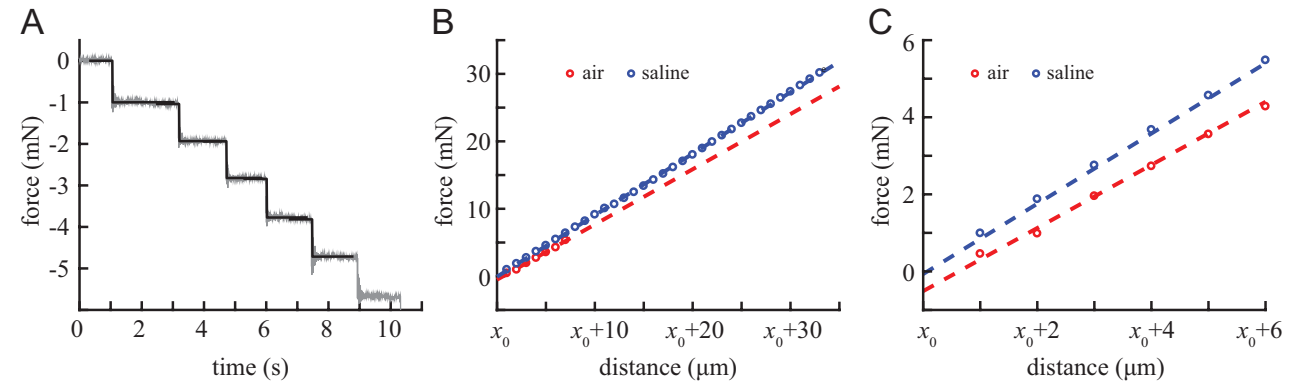

Fig. S8. Linearity of the force transducer measurements. (A) Raw data (grey) with superimposed fits (black) of two perpendicular razorblades pressed against each other. (B) Force-deflection graphs for measurements in air (red) and saline (blue). The vertical offset between the curves is due to the difference in initial conditions (i.e. the vicinity of the razor blades before the step in which contact was made). (C) First six deflection steps from the graphs shown in (B). The dynamic range of the force transducer used in the experiments is $0 \mathrm{~g}-10 \mathrm{~g}$, with a resolution of $<1 \mathrm{mg}(=0.01 \mathrm{mN})$.

\section{SI Curvature induced in three point bending experiments}

We estimated the mid-plane curvature of the samples during bending tests using [48]:

$$
d_{(x)}=\frac{F_{(x)}}{48 E I}\left(4 x^{3}-3 x L^{2}\right)
$$

where $x$ is the distance along the beam from one of the lateral pins, $d_{(x)}$ is the deflection of the sample, $F_{(x)}$ is the force needed for that deflection, $E$ is the Young's modulus of the sample, $I$ is its second moment of area, and $L$ is the distance between the outer pins used for three point bending (Fig. S7A). The second derivative of 


\section{II}

equation S8 is a good approximation of the induced curvature of the mid-plane of the beam $\left(\kappa_{\text {mid }}\right)$, when the deflections are small. To compare the curvature of structures with different diameters, dimensionless curvature can be used: $\kappa_{\text {mid }}^{*}=$ $\kappa_{\text {mid }} w_{\mathrm{s}}$, where $w_{\mathrm{s}}$ is the width of the sample in the bending direction. For small deflections (i.e. $\kappa_{m i d}^{*} \ll 1$ ), we can use the following formula to calculate $\kappa_{m i d}^{*}$ for a given deflection:

$$
\kappa_{\text {mid }}^{*}=d_{(x)}^{\prime \prime} w_{\mathrm{s}}=\frac{F_{(x)} x}{2 E I} w_{\mathrm{s}}
$$

For $x=\frac{L}{2}$ and using the effective bending stiffness estimated from the first full deflection step ( $E_{\text {eff }} I_{x} ;$ Fig. $3 \mathrm{~B}$ ) instead of $E I$, we obtain the following equation:

$$
\kappa_{\text {mid }}^{*}=\frac{F_{(d)} L}{4 E_{\mathrm{eff}} I_{\mathrm{x}}} w_{\mathrm{s}}
$$

where $F_{(d)}$ is the force recorded in a given deflection step, namely $10 \mu \mathrm{m}, 20 \mu \mathrm{m}$ or $30 \mu \mathrm{m}$. For whole ovipositors, $w_{\mathrm{s}}=30 \mu \mathrm{m}$, for ventral valves $w_{\mathrm{s}}=20 \mu \mathrm{m}$, and for dorsal valves $w_{\mathrm{s}}=10 \mu \mathrm{m}$.

We compared the curvature induced in the three point bending experiments to those observed during natural probing. ${ }^{4}$ The maximum dimensionless curvature of the whole ovipositor is $0.048 .{ }^{4}$ This value was adjusted to the size of ventral and dorsal valves by multiplying it with the diameter ratios ventral valve/whole ovipositor and dorsal valve/whole ovipositor, respectively. The same valve diameters were used as above. The dimensionless curvature induced in the first full deflection in three point bending experiments was similar to the one observed during kinematic experiments, whereas the second and third full deflection step usually induced higher curvatures than found in nature (Fig. S9B-D). ${ }^{4}$ The effective stiffness of the ovipositor and its valves were therefore estimated from the first full deflection step-that is, the deflection step after omitting the deflection where the razorblades were not in contact with the sample throughout the bending. 

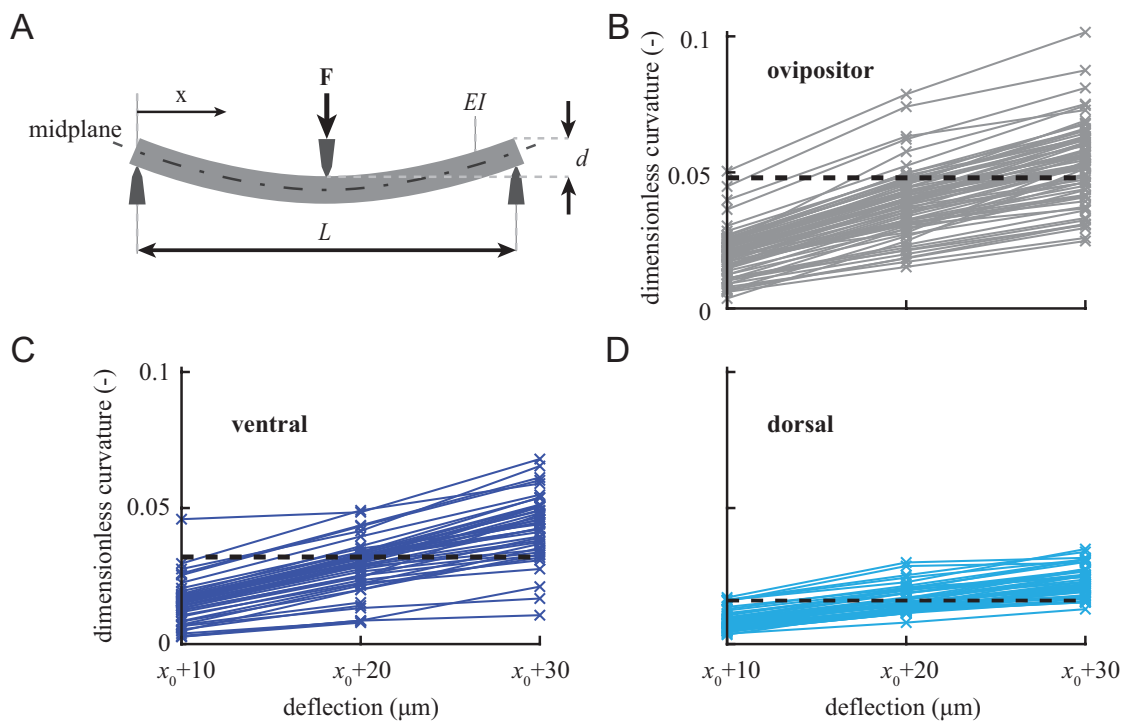

Fig. S9. Samples were during experiments bent in their natural range with extension. (A) Schematics of three point bending experiment with parameters used for estimating the curvature during bending from the recorded force. We estimated the mid-plane curvature of the sample assuming central loading. The estimated dimensionless curvature for the whole ovipositor (B), ventral (C), and dorsal valves (D) at each tested location along their length. The maximal dimensionless curvature observed in kinematic experiments is indicated with a horizontal black dashed line. The dimensionless curvature of the whole ovipositor (0.048) was taken from [4] and adjusted for the diameters of individual valves. In the first full deflection step, one whole ovipositor sample, one ventral valve sample, and two dorsal valve samples slightly exceeded the maximal values recorded in vivo. In the second deflection step, seven whole ovipositors and approximately half of the single ventral and dorsal valves exceeded the natural range. In the third deflection step, more than half of the samples exceeded their natural bending range.

\section{SI Videos}

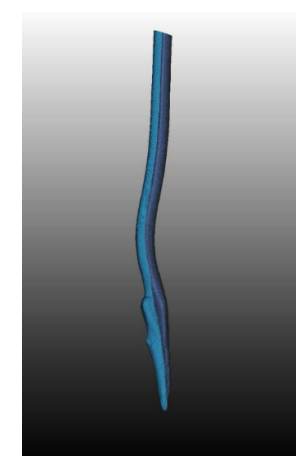

Video S1. Segmented $\mu \mathrm{CT}$ scan of the ovipositor. Colour code same as in graphs (Fig. 3); dorsal valve is light blue and ventral valves are dark blue. The characteristic S-shaped region of the ovipositor, the bevel shaped tips, the widening of the dorsal valve, and the serrations on the ventral valves are clearly visible. Additionally, tongue-and-groove mechanisms on the inner sides of the ovipositor are straight and do not show morphological variations. 


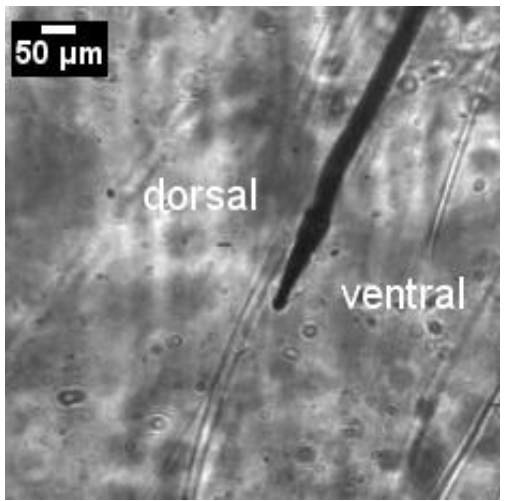

Video S2. Curved ovipositor insertion in the substrate. Protraction of ventral valve(s) within the substrate leads to dorsal curving of the ovipositor distal end. Video obtained as described in [4]. Video showing insertion in $2 \%$ gelatine, slowed down approximately $10 \times$.

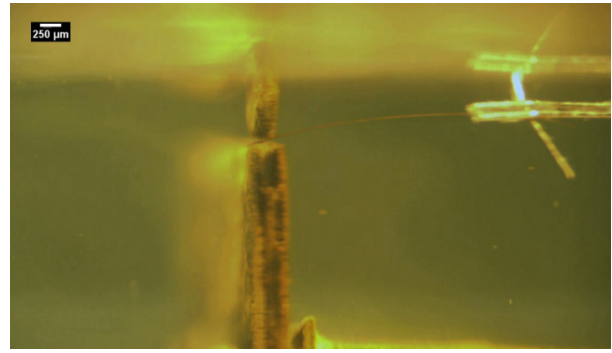

Video S4. Example movie showing part of the three point bending experiment.

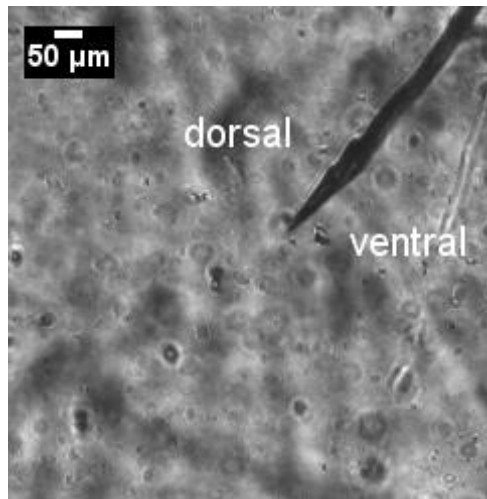

Video S3. Straight ovipositor insertion in the substrate. Protraction of dorsal valve within the substrate does not lead to noticeable curving of the ovipositors, which therefore makes a straight path. Video obtained as described in [4]. Video showing insertion in $2 \%$ gelatine, slowed down approximately $10 \times$.

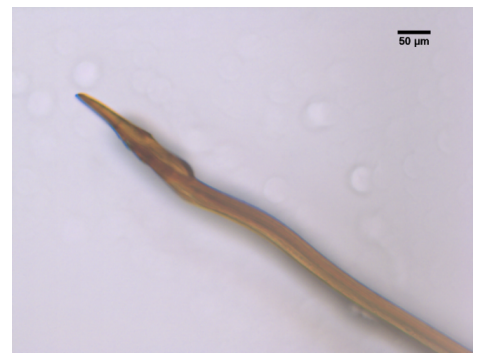

Video S5. Valve movements outside the substrate. Spontaneous valve movements outside the substrate observed under microscope-the animal was not in the probing position and a cover glass was put on top of the ovipositor to keep it in the focal plane. Protraction of both the dorsal and the ventral valves causes dorsal curving of the ovipositor distal end. Video recorded with Leica DFC $450 \mathrm{C}$ camera (5MP) mounted on Leica DM6b microscope (Leica Microsystems). Slowed down approximately $4 \times$. 



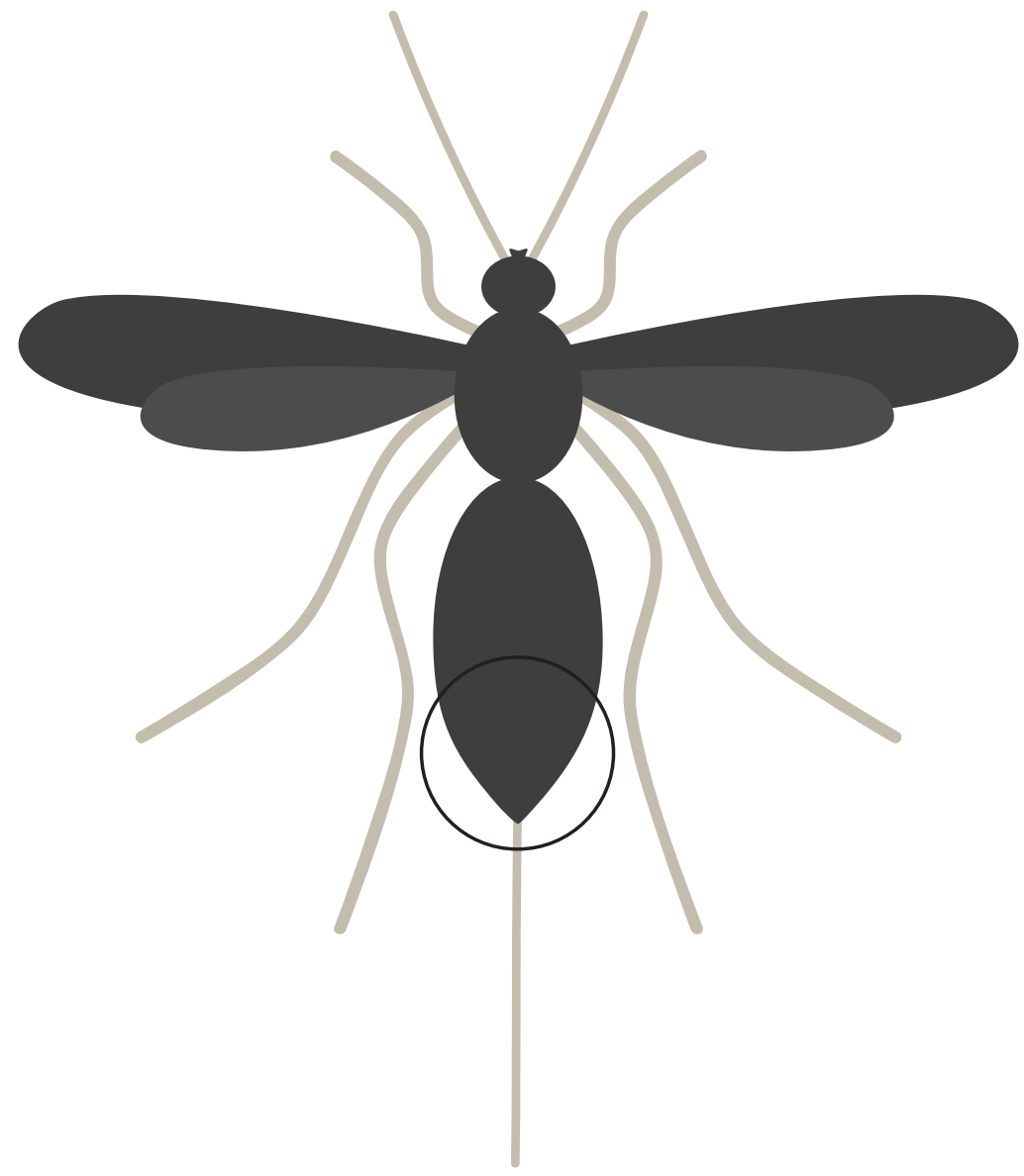




\section{The ovipositor actuation mechanism of a parasitic wasp}

Noraly M.M.E. van Meer ${ }^{1}$, Uroš Cerkvenik ${ }^{1}$, Christian M. Schlepütz ${ }^{2}$, Johan L. van Leeuwen ${ }^{1}$, and Sander W.S. Gussekloo ${ }^{1}$

${ }^{1}$ Experimental Zoology Group, Department of Animal Sciences,

Wageningen University, De Elst 1, 6708 WD Wageningen, The Netherlands

${ }^{2} \mathrm{X}$-ray Tomography Group, TOMCAT Beamline, Paul Scherrer Institute, WBBA/214, 5232 Villigen-PSI, Switzerland 


\section{Abstract}

Parasitic wasps use specialized needle-like structures, ovipositors, to drill in substrates to reach hidden hosts. The external ovipositor consists of three interconnected, sliding elements (valves) which are moved reciprocally during insertion. This presumably reduces the required outside pushing force and limits the risk of damage whilst probing. It was still unclear how valves are actuated and what forces are generated during probing. We used synchrotron X-ray microtomographs to reconstruct the actuation mechanism of the parasitic wasp Diachasmimorpha longicaudata in four distinct phases of the probing cycle. We show that only the paired ventral valves of the ovipositor move independently, while the dorsal valve moves with the abdomen. The ventral valve movements are initiated by rotation of one chitin plate (valvifer) with respect to another such plate. This is achieved indirectly by muscles connecting the non-rotating valvifer and the abdominal ninth tergite. Unlike previously reported, we found muscles running inside the ovipositor although the function remains unclear. The estimated maximal muscle forces are small and lead to small forces of the ventral valves, which may indicate that small push forces are used during probing. Our findings improve the comprehension of hymenopteran probing and the function of the associated muscles. 


\section{Introduction}

Reproduction is one of the most important elements in the life history of animals. In many species, we therefore see behaviour and adaptations that increase the reproductive success. Many insects, for example, hide their eggs in substrates that provide food and protection for the developing larvae. ${ }^{1}$ Many parasitic wasps go a step further and lay their eggs in larvae of host species that are already hidden deep within substrates such as fruits and wood. ${ }^{2-4}$ To reach these hosts, wasps drill into the substrate with long and thin (slender) ovipositors (Fig. 1A). ${ }^{5,6}$ Not only can these animals penetrate the often stiff substrates, but they can also steer their ovipositors to reach the desired targets. 3,6 This probing behaviour is a challenging task as slender drilling structures can easily buckle and get damaged due the substrate reaction forces. However, parasitic wasps clearly avoid damaging the ovipositor, as most wasps lay eggs in multiple hosts. ${ }^{7}$ A good understanding on how parasitic wasps avoid damage to their ovipositors, and how they drill and steer in often tough substrates is relevant in many aspects. Not only does it provide insight in the adaptation and co-evolution occurring in the group of hymenoptera, but this knowledge can also be applied in man-made steerable probes such as used in the medical profession.

To fully understand the working mechanism of the ovipositor, a general knowledge of its morphology is essential. The insect ovipositor originally consisted of four elements-two ventral valves (also called 'first valvulae') and two dorsal valves (also called 'second valvulae'), but reduction in the number of elements occurred during evolution of various insect taxa. ${ }^{9}$ In parasitic wasps, the two dorsal valves are generally merged, which results in ovipositors with three functional elements (Fig. 1B). ${ }^{10-12}$ These ventral and dorsal valves are longitudinally interconnected with a tongue-and-groove (olistheter) mechanism that allows for longitudinal sliding of the elements, while preventing their separation (Fig. 1B). ${ }^{12-14}$ It has been hypothesized that during probing many insects move these valves in saw-like fashion, $12,13,15$ which has recently been confirmed for a parasitic wasp. ${ }^{6}$ The reciprocal movements of the valves are thought to play an important role in buckling avoidance as they facilitate the so-called push-pull mechanism. ${ }^{15}$

According to this mechanism, buckling is avoided by pushing only certain valves, while simultaneously pulling on the others fixed in the substrate. ${ }^{15,16}$ The tension in the latter valves increases their flexural stiffness, which allows them to serve as guides for the valves that are pushed inside the substrate. ${ }^{15}$ The reciprocal movements appear crucial for penetration of solid substrates, particularly when dealing with stiff substrates as shown for the parasitic wasps Diachasmimorpha longicaudata 


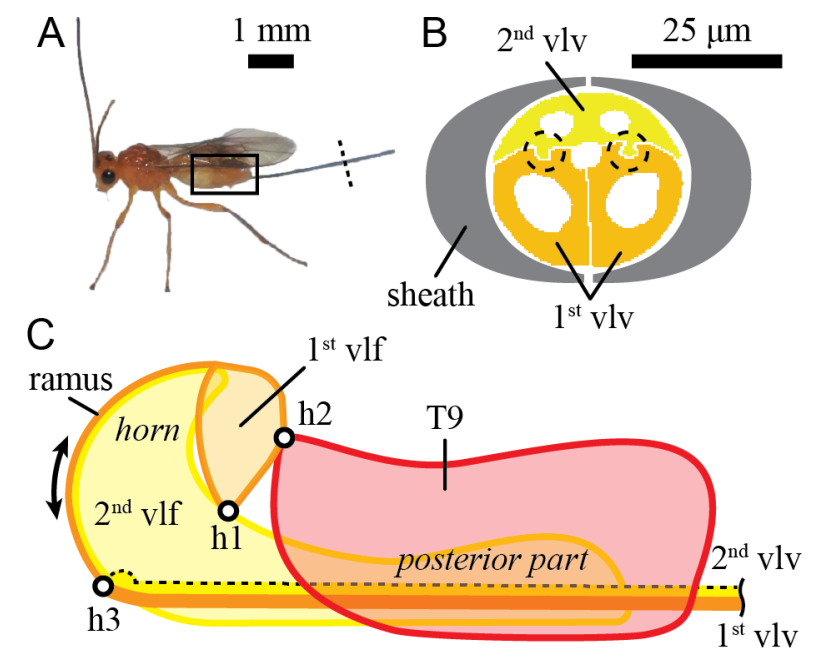

Fig. 1. Ovipositor apparatus of a parasitic wasp. (A) Parasitic wasp Diachasmimorpha longicaudata. The dotted line roughly indicates the location of the transverse section through the ovipositor shown in (B) and the rectangle roughly indicates the location of the ovipositor apparatus shown in (C). (B) A schematic cross-section of the ovipositor of $D$. longicaudata (at dashed line in (A)). $1^{\text {st }}$ vlv: ventral valves, $2^{\text {nd }}$ vlv: dorsal valve, black, dashed circles: the interlocking olistheter mechanism, sheath: sheaths surrounding the ovipositor. (C) Schematic representation of the left side of the apocritan ovipositor apparatus (based on [8]). All elements have a mirror image on the right side, apart from the dorsal valve ( $2^{\text {nd }}$ vlv), which is a single bilateral symmetric element located in the median. T9: the ninth abdominal tergite, $2^{\text {nd }}$ vlf: second valvifer that consists of an anterior horn and a posterior rectangular part. It is connected to the dorsal valve $\left(2^{\text {nd }} v l v\right)$. 1st vlf: first valvifer that is continuous with the ventral valve $\left(1^{\text {st }}\right.$ vlv $)$ via a ramus. The first valvifer hinges on the second valvifer at h1 and with $\mathrm{T} 9$ at h2. The external ovipositor can rotate in the medial plane around h3.

Ashmead (Braconidae), which always uses this mechanism in stiff substrates, but not in softer ones. ${ }^{6}$

In addition to simplifying insertion in stiff substrates, it has also been hypothesised that the movement of individual valves plays an important role in steering of the probe. ${ }^{6,17-19}$ In D. longicaudata, it has been shown that predominant protraction of the ventral valve leads to curved trajectories, while predominant protraction of the dorsal valves leads to straight insertions. ${ }^{6}$ Although knowledge about the kinematics of the valves is increasing, the equally important actuation of the valves is currently less well understood. Knowledge of the amplitude of the movements of the valves and the magnitude of the forces that can be exerted by the animals will provide insight in maximal performance and thus level of adaptation.

Extensive morphological descriptions of the ovipositor apparatus and its articulations can be found in the literature.$^{9-12,14,20,21}$ It is clear that the muscles that move the valves are positioned inside the abdomen, at the base of the valves. ${ }^{6}$ Inside the abdomen, the valves attach to plate-like basal exoskeletal elements (valvifers) that 
evolved from the coxae of the eighth and ninth abdominal segments. ${ }^{9,11,14,21,22}$ The valvifer shapes, arrangements, and articulations differ across insect species, ${ }^{14,20,23}$ but are similar across hymenopterans (Fig. 1C). ${ }^{8,9,11,24,25}$ The ventral valves attach via long rami to the usually triangular first valvifers $\left(1^{\text {st }}\right.$ vlf $),{ }^{11}$ while the fused dorsal valve attaches via smaller rami to two large second valvifers ( $2^{\text {nd }}$ vlf). ${ }^{9}$ The tongue-and-groove connection of the valves extends over the rami and the anterior ridge of the second valvifer. ${ }^{14}$ The second valvifers often bear at their posterior ends a pair of ovipositor sheaths that envelop the ovipositor in the rest position and may help to stabilise the ovipositor during probing (Fig. 1B). ${ }^{6,26}$ The first and second valvifers are linked with a hinge that allows for rotation of the first valvifer (h1 in Fig. 1C). ${ }^{9,11}$ A second hinge is present between the first valvifer and the ninth tergite (T9) of the abdomen ( $\mathrm{h} 2$ in Fig. 1C). ${ }^{11,14} \mathrm{~A}$ third, harder to recognise, hinge is located at the base of the valves and allows for rotation of the external ovipositor in the medial plane (h3 in Fig. 1C).

Current knowledge about the functioning of the ovipositor apparatus is solely based on morphological descriptions of dead specimens in resting position, with

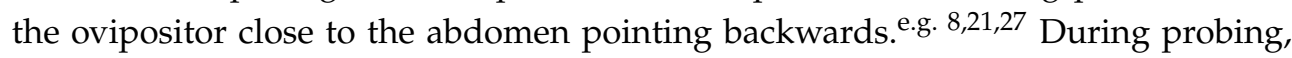
the ovipositor is rotated downward into probing position, which presumably results in significant changes in the configuration of the basal elements of the ovipositor. It is therefore not certain how any of the elements move during probing, apart from the sliding motion of the valves which has recently been visualised and quantified. ${ }^{6}$ The existing consensus is, that the external ovipositor as a whole can be pivoted downwards around h3 to get it in probing position. Protraction of the ventral valves is achieved by rotation of the first valvifer, which in turn is induced by pulling the second valvifers and T9 closer together. Retraction of the ventral valves is achieved by moving the second valvifers and T9 further apart. 8,13 This movement pattern is, however, completely theoretical and has never been recorded or quantified.

How the valvifers are moved and what forces act on them is also currently unknown. The musculature of the ovipositor apparatus differs across species. For example, in bees (fam. Apidae), orussid wasps (fam. Orussidae), and ceraphronoid wasps (fam. Ceraphronoidea), the first valvifer has muscular attachments with the outer exoskeleton of the animal's abdomen, ${ }^{9,28,29}$ while in cynipid wasps (fam. Cynipoidea) and chalcid wasps (fam. Chalcidoidea) such muscles have not been reported. ${ }^{8,21}$

Analysing the actuation mechanism of the ovipositor remains a major challenge. Visualization of the kinematics of the ovipositor base in vivo is extremely difficult, because it is hidden inside the abdomen and is in most species very small. As men- 
tioned, existing studies only describe the system with the ovipositor in resting position, which may be strikingly different from the probing situation. Furthermore, the relative positions and orientations of the valvifers and the T9 have not been quantified, and there is currently no data on the force production of the ovipositor base musculature.

In this study, we aim to elucidate a number of these issues by analysing the ovipositor apparatus of the parasitic wasp D. longicaudata for which the valve kinematics has been quantified. ${ }^{6}$ We used high-resolution synchrotron X-ray microtomography and $3 \mathrm{D}$ anatomical analysis to determine the configuration of the probing apparatus in different phases of the probing cycle. This allowed us to derive the kinematics of the complete probing cycle and calculate the range of motions of the valvifers and the valves. We also measured the physiological cross sectional area (PCSA), attachments, and the moment arms of the actuating muscles, which allowed us to estimate the forces acting on the valves. As mentioned above, this brings novel insights into the maximal performance of these animals and their adaptations. In addition, it will add to the understanding of probing with slender structures, which may be applied in man-made probes.

\section{Materials and methods}

\section{Animals}

A total of nine parasitic wasps of the species D. longicaudata were obtained from a colony maintained with the breeding protocol as described in [6] at the Experimental Zoology Group at Wageningen University and Research (Wageningen, Netherlands).

\section{Body positions and valve configurations}

To recreate a full drilling cycle, we prepared wasps with the ovipositor in two body positions and three valve configurations (Table 1): (1) in resting position with the valves aligned at the tips (aligned valves), (2) in probing configuration with aligned valves, (3) in probing configuration with the retracted ventral valves, and (4) in probing configuration with protracted ventral valves.

To obtain the probing configurations, live parasitic wasps were offered a gel-filled cuvette containing a Mediterranean fruit fly larva (Ceratitis capitata Wiedemann) at the bottom, as described in [6]. After the wasps started probing and when the ovipositor was fully inserted in the substrate, the cuvette and the wasp were quickly 
submerged in liquid nitrogen to preserve the wasp's body shape and position. Preliminary analysis showed that in all cases this resulted in ovipositors with retracted ventral valves. To obtain the other phases of the probing cycle, we thawed the wasps and used fine tweezers to slide the valves in the desired configuration, while keeping the body position intact.

For the resting position, two wasps were put inside a histology embedding cassette and frozen in liquid nitrogen, similar to the treatment of the probing animals. The valves of all these specimens were kept in place with a droplet of beeswax to preserve their configuration during the staining process (see below).

As a control for staining and freezing effects in the treated specimen, one wasp was decapitated, and scanned within minutes of decapitation with the ovipositor in resting position.

\section{Fixing and staining}

All wasps, except for the control, were stained with iodine for increased contrast in the CT-scans, according to either the IKI or $\mathrm{I}_{2}$ E staining protocols (30). In both protocols, the wasps were thawed for approximately one minute at room temperature before fixing in Bouin's solution overnight. Afterwards, the head and thorax were cut off to facilitate the entry of the staining solution into the abdomen. In the IKI protocol, the specimens were washed with 70\% ethanol and in three steps $(50 \%$ and $30 \%$ ethanol) transferred to distilled water with $0.05 \%$ Tween 20 . The specimens were then stained in a $10 \%$ IKI solution according to [30] for at least 7 days at $7{ }^{\circ} \mathrm{C}$. In the $\mathrm{I}_{2} \mathrm{E}$ protocol, the specimens were transferred in four steps $(80 \%, 90 \%$ and $98 \%$ ethanol) to $100 \%$ ethanol and then stained in an $\mathrm{I}_{2} \mathrm{E}$ solution $\left(1 \% \mathrm{I}_{2}\right.$ in $100 \%$ ethanol) for at least 7 days at room temperature according to [30]. During staining, both IKI and $\mathrm{I}_{2} \mathrm{E}$ solutions were refreshed at least once a day, but more often in the first day of staining. After staining, the samples were washed with distilled water and mounted on carbon fibre rods with beeswax in order to secure them on the rotation platform of the CT scanner. The staining affected the samples differently and we selected the samples that showed the best tissue contrast for further analysis. The IKI staining was usually more successful, although for the probing position with aligned valves the I2E stained sample was used (Table 1).

\section{Synchrotron X-ray microtomography}

High-resolution $\mu \mathrm{CT}$ scans were acquired at the TOMCAT beamline X02DA of the Swiss Light Source facility (Paul Scherrer Institute, Switzerland). The scans were made with $18 \mathrm{keV}$ (control) and $11 \mathrm{keV}$ (all other scans) monochromatic X-ray 
beams. Projection images were recorded over an angular range of $180^{\circ}$ with an angular step of $0.1^{\circ}$ with a PCO Edge $5.5 \mathrm{sCMOS}$ camera (exposure time of $100 \mathrm{~ms}$ ), using a $20 \mu \mathrm{m}$ thick LuAG:Ce scintillator. Whole abdomen scans were made at 20x magnification (resting configuration) and $10 \times$ magnification (probing configurations), resulting in effective pixel widths of $325 \mathrm{~nm}$ and $650 \mathrm{~nm}$, respectively. ${ }^{31}$ The scans were reconstructed using the gridrec reconstruction algorithm ${ }^{32}$ together with propagation-based phase contrast $(\delta / \beta=20)$ as described by Paganin et al.. ${ }^{33}$

\section{Analysis}

\section{Segmentation}

The reconstructed image stacks were processed with MeVisLab 2.8.2 (MeVis Medical Solutions AG, Bremen, Germany). The contrast between tissues was increased using edge enhancement and by subtracting the original reconstruction from the edgeenhanced images. A rough segmentation was done by applying a simple threshold filter, such that all relevant structures were retained, while removing as many other structures as possible. This segmentation was improved by manually delineating the individual structures of interest at locations with low contrast. Finally, segmented elements were further improved by applying morphological dilation and erosion filters which removed small artefacts and smoothed the outer boundaries of the elements (MeVisLab segmentation algorithm available on Dryad).

The tissue contrast in the unstained wasps was low and only two muscles were segmented using MeVisLab, and subsequently used for correction for staining and freezing artefacts. Two stained specimens showed low contrast and muscles were manually segmented using the paintbrush function in Seg3D 2.2.1 (University of Utah) ${ }^{34}$ as this program offers more effective tools for manual segmentations than MeVisLab (Table 1). All reconstructions were converted to polygon meshes for further visualization and measurements.

\section{Maximal muscle force production}

To get a rough estimate for the maximum forces that can be generated by the muscles moving the ovipositor and individual valves, we estimated the physiological cross sectional area (PCSA) of these muscles. In our analysis, we omitted effects of muscle length change and other muscle characteristics that may affect force generation. As most muscles were nearly parallel-fibred (see results) we used MeVisLab to manually orientate a plane through the widest part of the muscle reconstruction, perpendicular to the general direction of the muscle fibres. We used the combined 
Table 1: Overview of the animals and their treatments.

\begin{tabular}{|c|c|c|c|c|}
\hline \multirow[b]{2}{*}{ Valve configuration } & \multirow{2}{*}{$\begin{array}{c}\text { Resting position } \\
\text { aligned valves }\end{array}$} & \multicolumn{3}{|c|}{ Probing position } \\
\hline & & aligned valves & $\begin{array}{c}\text { retracted } \\
\text { ventral valve }\end{array}$ & $\begin{array}{c}\text { protracted } \\
\text { ventral valve }\end{array}$ \\
\hline Achieved by & natural & adjusted & natural & adjusted \\
\hline Preparation & $\begin{array}{c}\text { decapitation }(n=1) \\
\text { liquid nitrogen }(n=1)\end{array}$ & $\begin{array}{l}\text { liquid } \\
\text { nitrogen }\end{array}$ & $\begin{array}{l}\text { liquid } \\
\text { nitrogen }\end{array}$ & $\begin{array}{l}\text { liquid } \\
\text { nitrogen }\end{array}$ \\
\hline Staining & $\mathrm{IKI}^{\dagger, \ddagger}$, none $^{\dagger, \ddagger}$ & $\mathrm{I}_{2} \mathrm{E}^{\dagger, \ddagger}$ & $\mathrm{IKI}^{+}$ & $\mathrm{IKI}^{+}$ \\
\hline $\begin{array}{l}\text { Segmentation } \\
\text { software }\end{array}$ & $\begin{array}{c}\text { Seg3D + MeVisLab } \\
\text { MeVisLab }\end{array}$ & Seg3D + MeVisLab & MeVisLab & MeVisLab \\
\hline
\end{tabular}

${ }^{\dagger}$ specimen used for reconstruction of the exoskeletal elements

$\ddagger_{\text {specimen }}$ used for muscle reconstruction

cross-sectional area of the muscle fibres in this plain as a proxy for the physiological cross-sectional area (PCSA).

To enable comparisons between individual wasps, we scaled all measurements to dimensions of the control wasp. In the scaling, we assumed that chitin elements of the exoskeleton would not be affected by either staining or freezing, and therefore we scaled all reconstructions to the distance between easily identifiable landmarks on the second valvifer (for details see SI). Effects of freezing and staining were estimated based on the difference in estimated CSA after size correction of two muscles (for details see SI).

The maximal muscle force production was estimated by multiplying the CSA with a specific muscle tension of $195 \mathrm{kPa}$, Because data on abdominal musculature are missing, we used the average value of two other insect muscles: the femoral rotator muscle of the hind leg in the click beetle (Carabus problematicus; $210 \mathrm{kPa}{ }^{35}$ ) and the mandible closer muscles of the male stag beetle (Cyclommatus metallifer; $\left.180 \mathrm{kPa}^{36}\right)$.

\section{Torques and forces on the exoskeletal elements}

For the probing position with aligned valves, we determined the 3D orientations of rotation axes and positions of muscle attachment sites in the meshes of the reconstructed exoskeleton using Meshlab 1.3.2. ${ }^{37}$ A custom Matlab (R2016b) script was used to calculate the muscle moment arms $(r)$ from these coordinates. The maximal torques $(M)$ exerted by the muscles on the ovipositor or the first valvifer were estimated using the scalar version of the torque equation: $M_{i}=r_{i} \cdot F_{i}$, where $r_{i}$ is the moment arm of muscle $i$ with respect to the associated rotation axis and $F_{i}$ the estimated maximal force of muscle $i$.

The forces exerted on the substrate were estimated by dividing the maximal torques 
on the first valvifer with the moment arm of the ventral valves in the probing position with aligned valves (Table 2), using the same torque formula as above.

\section{Results and discussion}

\section{General morphology}

The general morphology of the ovipositor basal apparatus is similar to that of previously described species. ${ }^{\text {e.g. }}$ 9,11,14,20,29 Due to its bilateral symmetry, we will only present data for one side of the animals.

The whole ovipositor apparatus is attached to the abdomen via the tergite of the ninth abdominal segment (T9), This tergite articulates with a small triangular element (first valvifer) which is continuous with the ventral valve or first valve (Fig. 2). This connection (h2) seems strong, and allows for rotation in the sagittal plane. Ventrally (in resting position), the first valvifer articulates with a larger plate like element, the second valvifer (Fig. 2). This articulation (h1) allows for rotation in the sagittal plane as well. The second valvifer consists roughly of two regions: a rectangular posterior part and an anterior horn area. The second valvifer and T9 originate from the same abdominal segment ${ }^{22}$ and lie closely together, but do not touch (Fig. 2, Fig. S3A).

The needle-like external ovipositor or terebra consists of three elements; one dorsal valve, and two ventral valves. The ventral valves are connected to the dorsal valve via an olistheter mechanism that consist of a rail-like tongue (rachis) on the dorsal valve and a groove (aulax) in each of the ventral valves (Fig. 1B). This olistheter mechanism allows for longitudinal sliding of the valves, but prevents separation of the elements. The ventral valve extends anteriorly beyond the dorsal valve and forms an arched ramus that runs along the anterior edge of the horn of the second valvifer. The anterior edge of the horn, like the dorsal valve, carries a rachis, which acts as an extension of the olistheter mechanism. The rachis of the horn is continuous with the rachis on the dorsal valve via a small ramus that connects the two. This is a thin plate-like connection, and it is plausible that flexion in this area occurs during the downward rotation of the ovipositor as observed during probing (Fig. 3C). This movement is possibly stabilized by a shallow ball-and-socket-like articulation at the base of the second valvifer. The base of the dorsal valve is enlarged and divided into three processi: one medial and two lateral. The ventral valves run below and in between these processes (Fig. 2). The egg canal runs in between the three valves (Fig. 1B). 


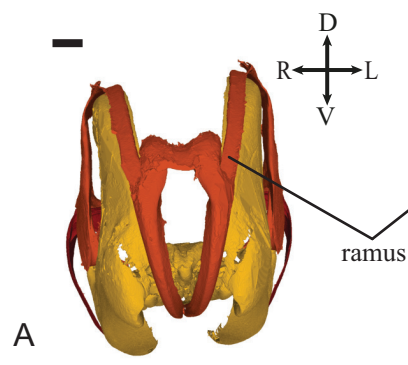

T9 latero-medial

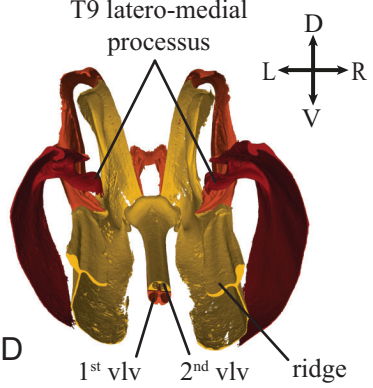

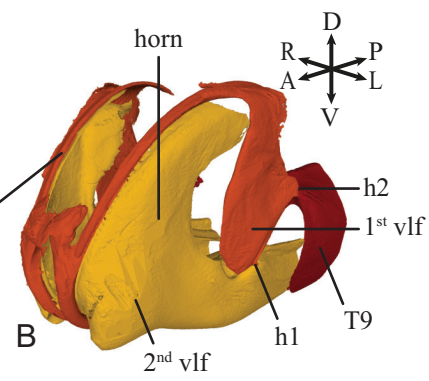

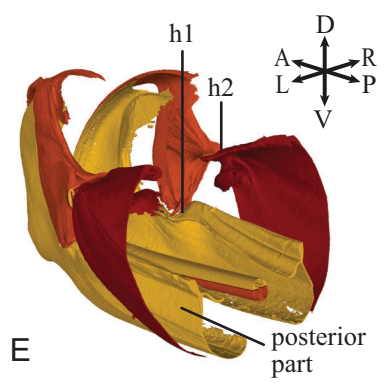

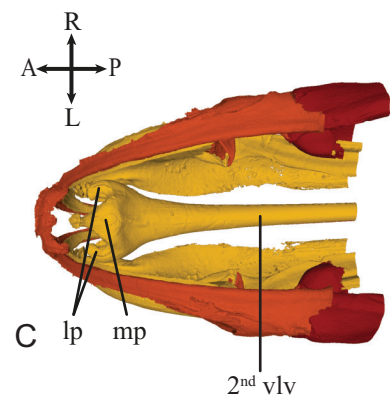

$\mathrm{A} \stackrel{\mathrm{L}}{\longrightarrow} \mathrm{P}$

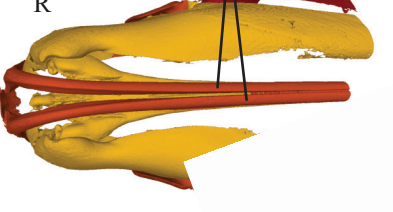

$\mathrm{F}$

Fig. 2. Three dimensional reconstruction of the ovipositor base of $D$. longicaudata in resting position with aligned valves. The colours indicate different exoskeletal elements. Orange: ventral valves $\left(1^{\text {st }}\right.$ vlv) and first valvifers $\left(1^{\text {st }}\right.$ vlf $)$; yellow: dorsal valve $\left(2^{\text {nd }} \mathrm{vlv}\right)$ and second valvifers $\left(2^{\text {nd }}\right.$ vlf $)$; red: tergum 9 (T9). The orientation of the base in each image is indicated with arrows: anterior (A), posterior (P), dorsal (D), ventral (V), left (L), and right (R). (A) Anterior view showing the long rami connecting the ventral valves with the first valvifers running along the anterior ridge of the second valvifer. (B) Anterior-lateral view. (C) Dorsal view showing the enlargement (bulbus) of the dorsal valve at its base, including the medial processus (mp) and two lateral processi (lp). (D) Posterior view showing the placement of the valves between the basal plates. E) Posterior-lateral view. (F) Ventral view showing the ventral valves ( $1^{\text {st }}$ vlv). Scale bar: $100 \mu \mathrm{m}$.

\section{Movements of the ovipositor basal apparatus}

\section{From resting to probing position}

Exoskeletal elements. When a wasps starts probing it elevates its abdomen and rotates the ovipositor downwards and forwards away from its resting position (Fig. 3B, C). We will call this rotation ovipositor depression, and the opposite movement ovipositor elevation. In our reconstructions, we observed a depression of the ovipositor of about $30^{\circ}$, but we expect that this can be more extreme. During ovipositor depression, the whole system of the basal plates rotates in the sagittal plane. This is presumably a result of the ventral curving of the abdomen (Fig. 3), but we did not investigate the musculature responsible for these movements.

Musculature. We identified two antagonistic muscle groups that can establish the depression and elevation of the ovipositor. Both muscles have their insertion on the dorsal valve. The posterior second valvifer-second valvula muscle (M12) ${ }^{25}$ inserts dorsally on the medial processus of the dorsal valve and fans out ventrally to the posterior part of the second valvifer (Fig. 4A, Fig. 4B top). The estimated 

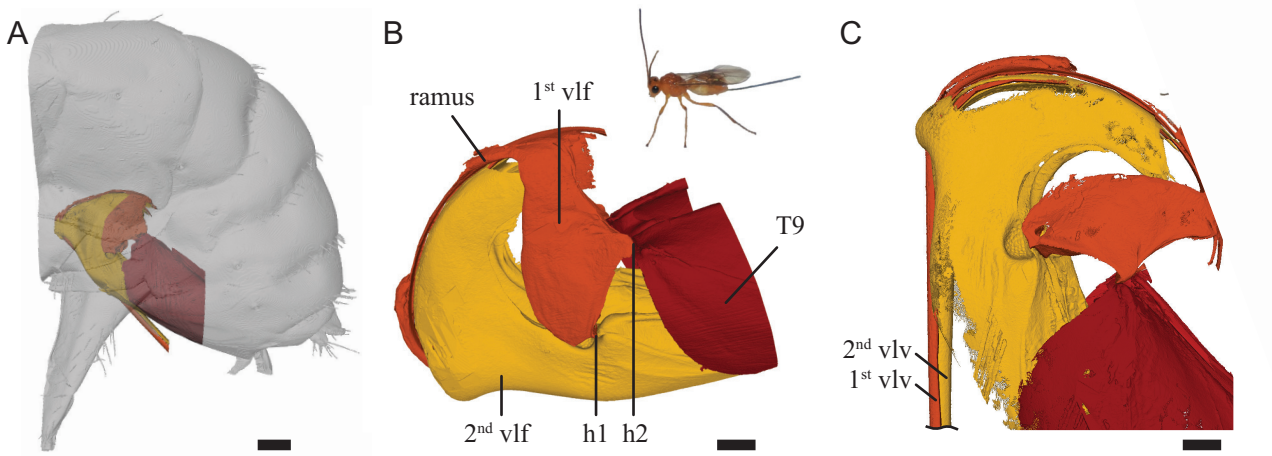

Fig. 3. Resting and probing positions of the ovipositor apparatus. (A) The ovipositor basal apparatus is located within the abdomen on its ventral side. Abdomen (grey) with the basal ovipositor (coloured) depicted in probing position with protracted ventral valves. (B) Top right: Wasp with ovipositor in resting position. Bottom: Side view of the configuration of the basal apparatus in the resting position with aligned valves. (C) Top right: Wasp in probing position. Bottom: Side view of the configuration of the basal apparatus in probing position. Scale bars: (A) $200 \mu \mathrm{m},(B, C) 100 \mu \mathrm{m}$ for basal apparatus only, size of wasp without ovipositor is about $5 \mathrm{~mm}$. See Fig. 2 for abbreviations.

maximum force generated by this muscle is $0.562 \mathrm{mN}$ (Table 2). The exact location of the rotation axis of the ovipositor (h3 in Fig. 1C) is difficult to determine and we assumed that this flexion point to be just anterior of the base of the dorsal valve. This is a short region where the valves are connected only with their rami, just proximal to the enlarged base of the dorsal valve (Fig. 2C, F). As mentioned, the muscle moment arms were determined for the wasp in probing position with aligned valves scaled to the size of the control. The estimated moment arm of M12 is $45.958 \mu \mathrm{m}$, which results in an estimated maximal torque of $26.058 \times 10^{-9} \mathrm{~N} \mathrm{~m}$ used to rotate the ovipositor into the probing position (Fig. $4 \mathrm{~B}$, top). Because the tendon of this muscle runs over the curved dorsal side of the base, the moment arm will probably change little over the range of motion of the ovipositor.

The anterior second valvifer-second valvula muscle (M11) ${ }^{25}$ connects the anterior inner wall of the second valvifer to the lateral processus of the dorsal valve and elevates the ovipositor into the resting position (Fig. 4A, Fig. 4B, bottom). We estimate M11 can provide a force of $0.577 \mathrm{mN}$ (Table 2) and would, with an estimated moment arm of $19.446 \mu \mathrm{m}$, generate a maximal torque of $9.811 \times 10^{-9} \mathrm{Nm}$ on the ovipositor. Angular changes, however, have a big impact on the moment arm of this muscle, and the observed rotation. Although our observed rotation of $30^{\circ}$ results in less than $15 \%$ reduction in torque, a rotation of $45^{\circ}$ will result in approximately a $30 \%$ reduction in torque. 

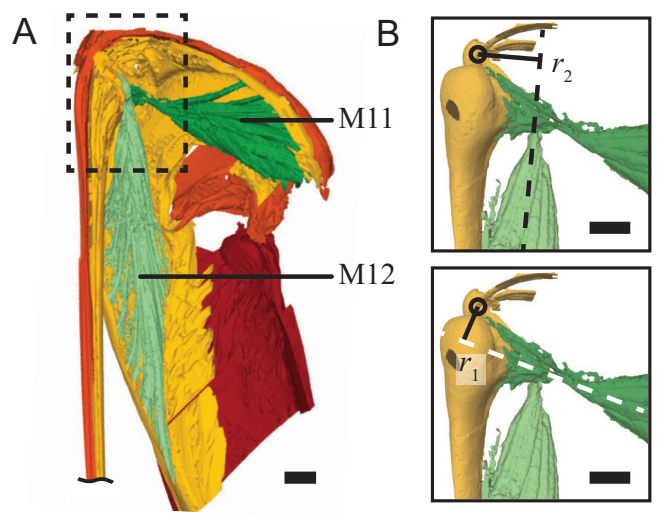

Fig. 4. Muscles that depress or elevate the ovipositor. Lateral view of the ovipositor apparatus in the probing position with aligned valves. (A) Two muscles attach directly to the anterior bulb of the dorsal valve. Muscle M12 connects the dorsal valve and the posterior end of the second valvifer and is presumably used to rotate the ovipositor towards the probing position (depression) (Fig. 7C). Muscle M11 connects the dorsal valve to the dorsal horn of the second valvifer and is presumably used to rotate the ovipositor towards the resting position (elevation) (Fig. 7F). (B) Enlarged view of the attachment sites of both muscles. Solid black lines $r_{1}$ and $r_{2}$ : moment arms of M11 and M12 respectively. Dashed lines: estimated lines of actions for both muscles. Centre of the black circles: estimated centre of rotation. For clarity, we show the high-resolution mesh of the dorsal valve in resting position which was fitted on the low-resolution mesh of the dorsal valve in probing position. Scale bars: $50 \mu \mathrm{m}$.

Table 2: Properties of the muscles associated with the ovipositor apparatus. Second column shows the location of the calculated physiological cross-sectional area (PCSA) for each muscle (red line). Third column show the animal's body position: $\mathrm{R}$ for resting position, $\mathrm{P}$ for probing position (both with aligned valves). The formulae used for calculating the muscle properties are provided in SI. The force estimates reported in the main text were obtained by averaging the values of the $\mathrm{P}$ and $\mathrm{R}$ positions for each muscle.

\begin{tabular}{|c|c|c|c|c|c|c|c|c|}
\hline & Muscle & $\begin{array}{l}\text { Cross-section } \\
\text { location }\end{array}$ & Position & $\begin{array}{c}\text { Measured } \\
\text { average PCSA } \\
\left(\times 10^{3} \mu \mathrm{m}^{2}\right)\end{array}$ & $\begin{array}{c}\text { Corrected } \\
\text { PCSA } \\
\left(\times 10^{3} \mu \mathrm{m}^{2}\right)\end{array}$ & $\begin{array}{l}\text { Force } \\
(\mathrm{mN})\end{array}$ & $\begin{array}{c}\text { Moment } \\
\text { arm } \\
(\mu \mathrm{m})\end{array}$ & $\begin{array}{l}\text { Torque } \\
(\mathrm{mN} \mu \mathrm{m})\end{array}$ \\
\hline M12 & $\begin{array}{l}\text { posterior second } \\
\text { valvifer-second } \\
\text { valvula }\end{array}$ & & $\begin{array}{l}\mathrm{P} \\
\mathrm{R}\end{array}$ & $\begin{array}{l}2.281 \\
1.627\end{array}$ & $\begin{array}{l}2.857 \\
2.909\end{array}$ & $\begin{array}{l}0.557 \\
0.567\end{array}$ & - & - \\
\hline M11 & $\begin{array}{l}\text { anterior second } \\
\text { valvifer-second } \\
\text { valvula }\end{array}$ & & $\begin{array}{l}\mathrm{P} \\
\mathrm{R}\end{array}$ & $\begin{array}{l}2.657 \\
1.445\end{array}$ & $\begin{array}{l}3.328 \\
2.584\end{array}$ & $\begin{array}{l}0.650 \\
0.504\end{array}$ & - & - \\
\hline & $\begin{array}{l}\text { medial second } \\
\text { valvifer-second } \\
\text { valvula }\end{array}$ & & $\begin{array}{l}\mathrm{P} \\
\mathrm{R}\end{array}$ & $\begin{array}{l}0.181 \\
0.072\end{array}$ & $\begin{array}{l}0.227 \\
0.130\end{array}$ & $\begin{array}{l}0.044 \\
0.025\end{array}$ & - & - \\
\hline M5 & $\begin{array}{l}\text { dorsal T9-second } \\
\text { valvifer }\end{array}$ & & $\begin{array}{l}\mathrm{P} \\
\mathrm{R}\end{array}$ & $\begin{array}{r}15.044 \\
7.432\end{array}$ & $\begin{array}{l}18.844 \\
13.291\end{array}$ & $\begin{array}{l}3.674 \\
2.5592\end{array}$ & - & - \\
\hline & $\begin{array}{l}\text { ventral T9-second va } \\
\text { medial muscle belly }\end{array}$ & & $\begin{array}{l}\mathrm{P} \\
\mathrm{R}\end{array}$ & $\begin{array}{l}7.964 \\
5.742\end{array}$ & $\begin{array}{r}9.976 \\
10.268\end{array}$ & $\begin{array}{l}1.945 \\
2.002\end{array}$ & $\begin{array}{c}- \\
-110.406\end{array}$ & - \\
\hline & lateral muscle belly & & $\begin{array}{l}\mathrm{P} \\
\mathrm{R}\end{array}$ & $\begin{array}{l}8.697 \\
6.271\end{array}$ & $\begin{array}{l}10.894 \\
11.214\end{array}$ & $\begin{array}{l}2.124 \\
2.187\end{array}$ & - & -157.230 \\
\hline M8 & $\begin{array}{l}\text { T9-genital } \\
\text { membrane }\end{array}$ & & $\begin{array}{l}\mathrm{P} \\
\mathrm{R}\end{array}$ & $\begin{array}{l}0.803 \\
0.501\end{array}$ & $\begin{array}{l}1.006 \\
0.896\end{array}$ & $\begin{array}{l}0.196 \\
0.174\end{array}$ & - & - \\
\hline
\end{tabular}


A

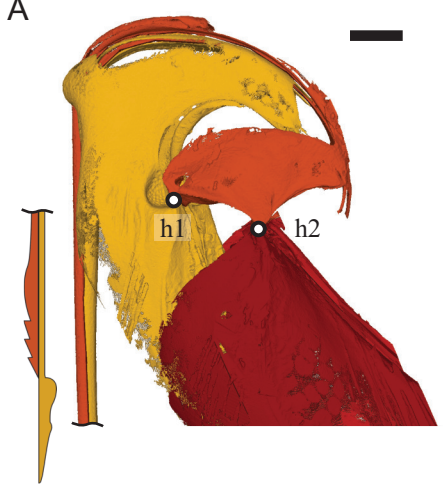

B

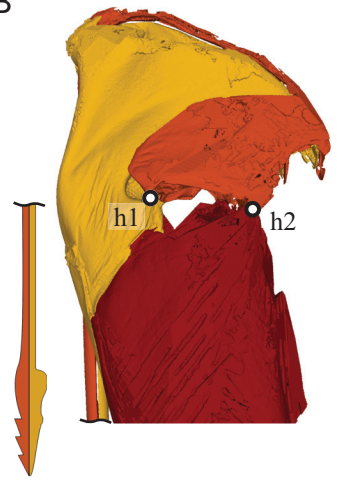

C

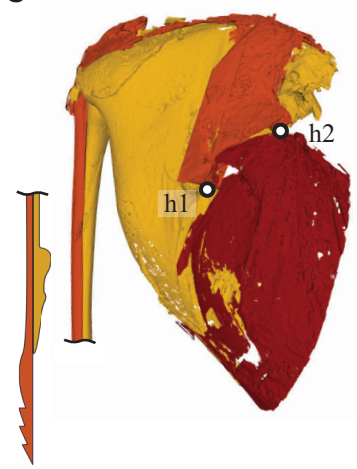

Fig. 5. Lateral view of the ovipositor apparatus in probing position with three different valve configurations. The first valvifer (orange) changes orientation during pro- and retraction of the ventral valves. The centre of rotation is at the inter-valvifer connection h1. Inserts show the relative position of the ventral valves (orange) and the dorsal valve (yellow). (A) Ventral valves in retracted state, the first valvifer have been rotated away from the horn of the second valvifer. (B) When the valves are (manually) aligned, the relative positions and orientations of the valvifers are similar to the resting position with aligned valves. (C) When the ventral valves are (manually) protracted, the first valvifers are rotated over the horn of the second valvifers. Scale bar: $100 \mu \mathrm{m}$.

\section{Valve motions during probing}

Exoskeletal elements. We analysed three distinct valve configurations to compose a working hypothesis about the kinematics and muscle activity during probing. As previously hypothesized by others ${ }^{\text {e.g. }} 13,14,24,27$ ), we observed a clear rotation of the first valvifer around its articulation with the second valvifer (h1) with changing alignment of the ventral and dorsal valves (Fig. 1C, Fig. 5). Moving the ventral valves from their complete retraction (offset $-237 \mu \mathrm{m}$ ) to complete protraction (offset $121.7 \mu \mathrm{m}$ ) corresponds to the rotation of the first valvifer of approximately $56^{\circ}$ (Fig. 5, Table 3). The calculated excursion path of the first valvifer along the anterior horn of the second valvifer with this angular change is $252.6 \mu \mathrm{m}$, which less than the estimated excursion of the ventral valve tip ( $349.7 \mu \mathrm{m}$; Table 3$)$, indicating that the preparation of the specimens might have affected the configuration of the basal plates or the shape of the ovipositor distal end. Nevertheless, the difference is still very small compared to the size of the entire ovipositor apparatus.

According to the hypothesized mechanism, the movement of the ventral valves and first valvifers result from the changing positions of the second valvifer and T9. e.g. 8,14,24,38 This is also visible in our reconstructions (Fig. 5). The second valvifer and T9 telescopically slide away from each other when the ventral valves are retracted and towards each other when the ventral valves are protracted.

Musculature. In the following description of movement, we use the second valvifer as our reference element to which all movements of the other elements are 
Table 3: Movement of the first valvifers and ventral valves. The angles $\left(\alpha_{\mathrm{i}}\right)$, radii $\left(r_{\mathrm{i}}\right)$, and excursion paths (arc lengths, $s_{i}$ ) were determined for all segmented probing positions and compared to the corresponding valve offsets $\left(o_{\mathrm{i}}\right)$. The image shows the points $\mathrm{P}$ (reference), h1, and h2 chosen as landmarks for calculating the angles and arc lengths in segmentations. The point h2 changes location from h2A (ventral valve retraction) to $\mathrm{h} 2 \mathrm{~B}$ (ventral valve protraction), which corresponds to the maximal change in angle $\alpha_{2}-\alpha_{1}$. Calculation of the arc lengths and the method for determining the displacement of the valves are given in the $S I$.

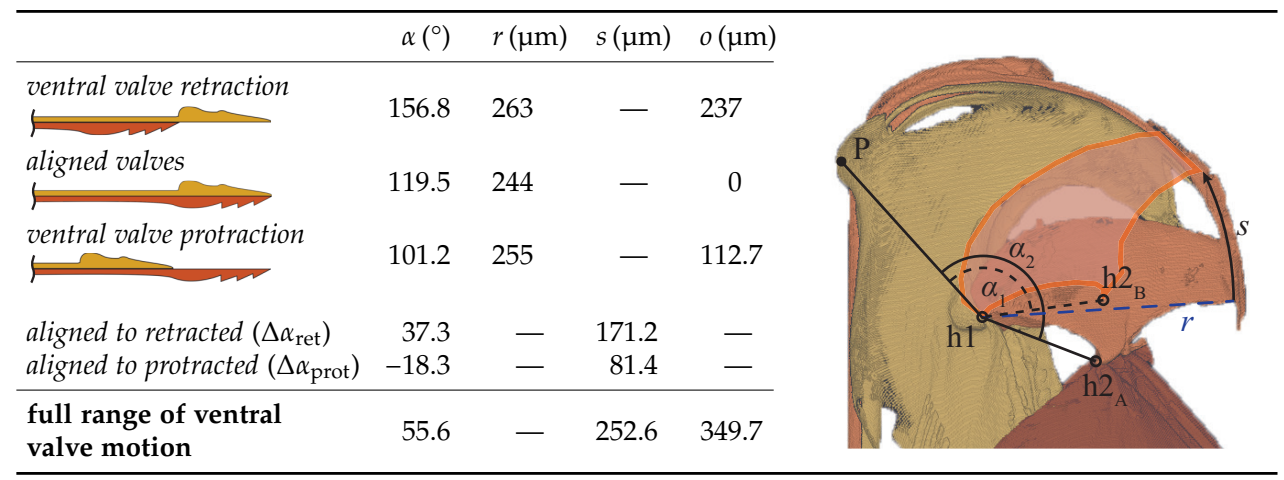

described. In reality, all elements can move relative to each other and the second valvifer does not remain stationary relatively to the external frame of reference. We identified three muscles connecting the second valvifer with T9, which can actuate the sliding movements of T9 relative to the second valvifer. The ventral T9-second valvifer muscle (M6, Fig. 6A) ${ }^{25,29}$ consists of two muscle bellies that originate at the anterior dorsal edge of $\mathrm{T} 9$ on a clear latero-medial oriented process extruding from the medial side of T9 and over the second valvifer (Fig. 2D, E). The inner muscle belly runs medial to the second valvifer and inserts on a plate on the medial side which increases the attachment area (Fig. 2D, E). The outer belly runs in between the second valvifer and T9 and inserts on the lateral wall of the second valvifer. It has additional fibres originating from the medial side of the T9 plate itself. The dorsal side of this part of the second valvifer has an enlarged ridge, which increases the attachment area and probably strengthens the second valvifer to oppose bending along its longitudinal axis in the dorsoventral direction. The estimated forces both muscle bellies can generate (medial: $1.974 \mathrm{mN}$, lateral: $2.156 \mathrm{mN}$ ) are similar (Table 2). Contraction of M6 slides T9 posteriorly relatively to the second valvifer and increases the distance between T9 and the second valvifer horn. This results in the posterior rotation of the first valvifer around h1 due to the connection between this plate and T9 at h2. The rotation of the first valvifer moves the rami and retracts the ventral valve. The estimated moment arms of the muscle bellies (Fig. 6A, $r_{3}$, shown only for one muscle belly) are $110.406 \mu \mathrm{m}$ (medial) and $71.891 \mu \mathrm{m}$ (lateral), which results in the maximal torques on the first valvifer of $221.033 \times 10^{-9} \mathrm{~N}$ m and $157.230 \times 10^{-9} \mathrm{Nm}$ (Table 2). The combined torques result in $378.263 \times 10^{-9} \mathrm{~N} \mathrm{~m}$ re- 
traction force that can be exerted on the first valvifer. In the full range of motion of the first valvifer the reduction in moment arm for this muscle is about $10 \%$.

The dorsal T9-second valvifer muscle (M5) ${ }^{25,29}$ originates on the tip of the horn of the second valvifer it runs dorsal of $\mathrm{T} 9$ on which it attaches on its posterior dorsal rim (Fig. 6A). ${ }^{25,28,29}$ This muscle is large, with an estimated maximum force of $3.133 \mathrm{mN}$ (Table 2). Contraction of this muscle pulls T9 closer to the second valvifer. This induces anterior rotation of the first valvifer around $\mathrm{h} 1$, which in turn results in movement of the rami and protraction of the ventral valve. The estimated moment arm (Fig. 6A, $r_{4}$ ) is $172.921 \mu \mathrm{m}$, which leads to a computed maximal torque of $448.211 \times 10^{-9} \mathrm{Nm}$ on the first valvifer. The moment arm changes about $10 \%$ during the entire range of first valvifer motions, so this muscle could potentially generate very similar torques and forces throughout the probing process.

\section{Additional musculature associated with ovipositor ap- paratus}

We identified two muscle groups that connect the left and the right sides of the ovipositor apparatus and which appear not to actuate the ovipositor. One muscle consists of only a few muscle fibres and originates on the anterior-latero-medial wall of the second valvifer, stretches over the sides of the lateral processes of the dorsal valve and inserts inside the lumen of this valve (Fig. 6A). As far as we are aware, this muscle has never been described, so to make sure we did correctly identify this structure as a muscle we did additional histological analysis which confirmed our findings (see SI, and Fig. S2). We propose to name this muscle the medial second valvifer-second valvula muscle (M-2vlf-2vlv). This tiny muscle can exert an estimated maximal force of $0.035 \mathrm{mN}$ (Table 2). The function of this muscle is uncertain because of its location just dorsally of the rami connecting the dorsal valve and second valvifer.

Another muscle pair originates on the medial side of the first valvifer near the hinge with the second valvifer (h1), interconnects medially, and could potentially link to the genital membrane, which was not clearly discernible in our scans (M8 ${ }^{25}$; Fig. 6B). This muscle comprises of few muscle fibres and may exert an estimated maximal force of $0.185 \mathrm{mN}$ (Table 2).

We also found two muscle groups that attach to the lateral side of the first valvifer (Fig. S3B). These muscles are small and appear not to play an important role in actuation. We segmented them only partially. One muscle is directed posteriorly and based on literature might connect the first and the second valvifers. ${ }^{25}$ We denote this muscle as M-1vlf-A (Fig. S3B). The other muscle (M-1vlf-B) is directed ventrally 
A

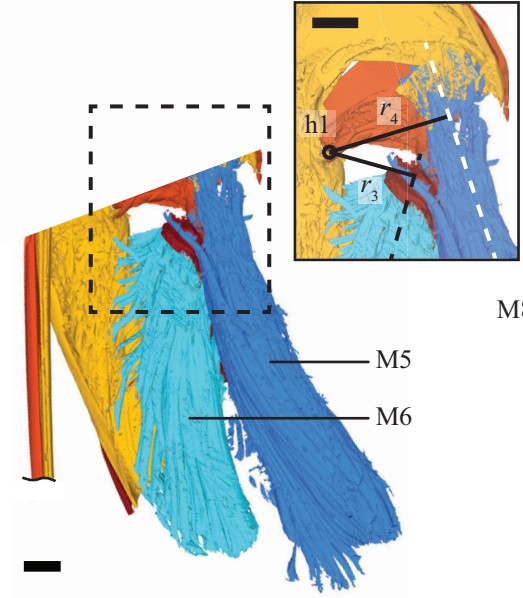

B

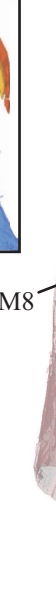

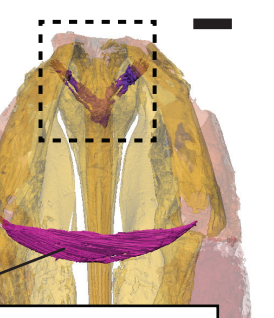

C

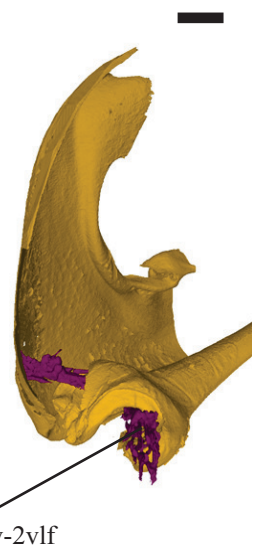

Fig. 6. Muscles actuating the reciprocal valve movements and stabilizing the ovipositor apparatus. (A) Lateral view of the ovipositor base in the probing position. The M6 muscle is fan shaped and splits into two muscle bellies that wrap around the second valvifer. This muscle connects the anterior part of T9 with the posterior part of the second valvifer and its contraction presumably slides these two exoskeletal elements away from each other. This results in rotation of the first valvifer posteriorly, causing retraction of the ventral valves (Fig. 5E). Muscle M5 connects the dorsal horn of the second valvifer with the posterior part of $\mathrm{T} 9$ and its contraction slides these two exoskeletal elements towards each other. This causes rotation of the first valvifer anteriorly, leading to protraction of the ventral valves (Fig. 5D). (B) A dorsal view of the system showing two muscles that connect its left and right sides. Muscle M8 connects near the first valvifer-T9 articulation, while the M-2vlv-2vlf muscle (inset) links the base of the dorsal valve to the second valvifer. (C) Antero-medial view of the dorsal valve base showing the location of the M-2vlv-2vlf muscle. Scale bars: $50 \mu \mathrm{m}$.

towards the abdominal wall (Fig. S3B), but we could not unambiguously determine its attachment site from our scans or literature. If we assess their potential contribution to valve movement, we must conclude that their moment arms are very short. M-1vlf-A runs near the axis of rotation h2, while M-1vlf-B spans across the axis of rotation of h1 (Fig. S3B). The moment arms of these muscles do increase when the ventral valves are offset and may than contribute slightly in the actuation of the valvifer, although they probably have a stabilizing function. ${ }^{8,9}$

\section{General discussion}

In the insect orders Odonata, Orthoptera, Hemiptera, and Hymenoptera, multielement structures (ovipositors) have evolved that allow for laying of the eggs in hard-to-reach places. ${ }^{9,14}$ Parasitic wasps often lay eggs in hosts hidden deep within substrates, for which they use long and slender ovipositors that are susceptible to damage during insertion. A recent study showed that wasps use alternating movements of the ovipositor valves when penetrating a stiff substrate, ${ }^{6}$ which hypothetically reduces the risk of buckling damage. ${ }^{15}$ The movement of the 
valves is initiated at their bases inside the abdomen. Although the morphology of this basal ovipositor apparatus and the muscle attachments have been extensively described, e.g. 9,10,20,24,27 no one has, as far as we are aware, quantitatively analysed the configurational changes of the basal ovipositor apparatus that occur during probing. Here, we reconstruct the movements in the ovipositor base using configurations from three distinct phases of the probing behaviour and the resting position. We also analyse the muscles that make these movements possible and estimate their maximum contraction forces. For these muscles, we describe the most obvious function when no other muscles are active. Combinations of muscle contractions or isometric contractions can lead to additional functions, but we do not consider those as we focus on clearly specified phases of the probing behaviour, which can all be explained by contractions of the muscles we discuss. Below, we describe these important phases of the probing behaviour.

\section{Probing mechanism}

\section{Moving between resting and probing positions}

Upon the start of probing, a female parasitic wasps raises its abdomen, curves it ventrally, and rotates (depresses) the ovipositor from its resting backward orientation to a downward orientation (Fig. 7A, B, C). ${ }^{5,6,39}$ From our analysis, it is clear that the entire ovipositor base rotates during the ventral curving of the abdomen as the angle between the first valvifer and T9 changes only slightly (Fig. 3). The depression of the ovipositor is presumably achieved by the muscle M12, which can pull the medial processus dorso-posteriorly and rotates the valves (Fig. 7C). ${ }^{8,9,24,28,40} \mathrm{We}$ could not accurately determine the maximal rotation angle of the ovipositor as this was probably affected during the fixing procedure and scanning itself. However, based on the reported probing process of parasitic wasps, the valves can rotate over a larger angle than observed here. Based on $[5,6]$, we estimate that the valves can rotate at least up to $60^{\circ}$ with respect to their basal plates. At the end of probing, when the animal completely extracts its ovipositor from the substrate, contraction of muscle M11 presumably pulls on the lateral processus of the dorsal valve anteriorly, rotating (elevating) the ovipositor into its resting position (Fig. 7F). ${ }^{11,15,17,30}$

\section{Valve motions during probing}

After puncturing the substrate, the wasps move the valves reciprocally during insertion. ${ }^{6,15}$ We did not find any musculature attaching directly to the valves that could generate such movements. Our findings are in agreement with previously proposed mechanism of probing, namely that movements of the valves are a result of the movements of their valvifers. $8,13,20,21,27,41$ 

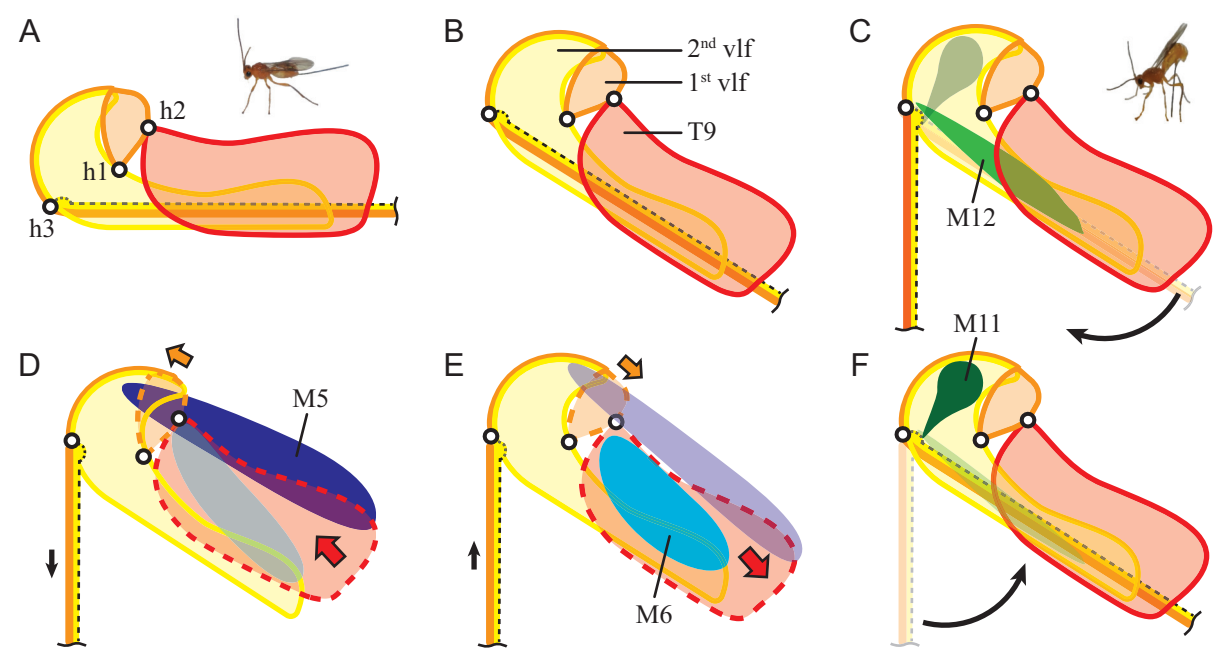

Fig. 7. Schematics of the probing mechanism in lateral view. Movements of the exoskeletal elements are shown in the reference frame of the second valvifer and are indicated with arrows. Movement is predominantly induced by the opaque muscles in each image. (A, B) The ovipositor apparatus is rotated from the resting to probing orientation. (C) The ovipositor is depressed from the abdomen using muscle M12. (D) Contraction of muscle M5 slides T9 towards the second valvifer, causing anterior rotation of the first valvifer and protraction of the ventral valves. (E) Contraction of muscle M6 slides T9 away from the second valvifer, causing posterior rotation of the first valvifer and retraction of the ventral valves. (F) The ovipositor is elevated from the probing to resting orientation using muscle M11.

Our morphological investigation shows that the movements of the dorsal valve are limited compared to the translations of the ventral valves. Although small forward motions of the dorsal valve cannot be excluded, we hypothesize that the dorsal valve is inserted predominantly by the motion of the complete abdomen. We found, however, a previously undescribed muscle inside the dorsal valve that may contribute to its functioning. The newly described M-2vlf-2vlv attaches inside the base of the dorsal valve, which is surprising as the ovipositors are always reported to be devoid of any musculature. ${ }^{10,42}$ The muscle does not seem to extend deeply inside the external ovipositor, is small, and cannot generate high forces. The position of M-2vlf-2vlv indicates that its contraction may pull the dorsal valve towards the second valvifer. This muscle can therefore pull on the dorsal valve and increase the tension in the valve, contributing to the push-pull mechanism, although with little force. This muscle may also be active during extraction of the ovipositor, thereby supporting the thin dorsal valve-second valvifer rami which might come under high tensile stresses during retraction of the ovipositor. Finally, activation of the individual left or right muscle bellies of this muscle may induce small rotational forces on the ovipositor, although this seems unlikely due to the small size of the muscle. 
In contrast, the movements of the ventral valves are initiated by muscles that act on the first valvifers, which cause a rotation around h1 with the second valvifer (Fig. 1C, Fig. 5; h1). The left and right first valvifers are connected via the M8 muscle. This muscle presumably also links to the medial genital membrane and might stabilize the overall configuration of the ovipositor base to avoid damage in the abdominal cavity. ${ }^{25}$ Additionally, M8 might contribute to bringing the ventral valves in their aligned configuration. Two other small muscles we report here attach also the medial side of the first valvifer (Fig. S3B). These muscles appear to have a very small effect on the rotation of the first valvifer, and are thought to only assist in moving the valves ${ }^{27}$ or serve as stabilizers. ${ }^{8}$

The movements of the ventral valves are likely powered indirectly by the muscles connecting the second valvifer to T9, as was also previously predicted. $8,13,20,21,24,27,41$ These muscles are big and are expected to generate the largest torques on the valvifers (Table 2). Contraction of muscle M5 causes anterior rotation of the first valvifers and protraction of the ventral valves (Fig. 7D). Contraction of muscle M6 causes posterior rotation of the first valvifer and retraction of the ventral valves (Fig. 7E). These torques lead to driving forces on the ventral valves in the range of $1.01 \mathrm{mN}$ $1.20 \mathrm{mN}$, using the estimated moment arm of the ventral valve scaled to the control specimen of $372.294 \mu \mathrm{m}$. How these forces relate to the forces experienced in the field is hard to determine because they depend on may environmental factors such as the condition of the probing substrate.

\section{Push-pull mechanism}

The alternating valve motions (push-pull mechanism) which are observed during drilling are hypothesized to reduce the risk of buckling during insertion by minimizing the net pushing forces on the ovipositor. ${ }^{15}$ Using Euler-Bernoulli beam bending theory, we can estimate the buckling threshold of the ovipositor of $D$. longicaudata. The ovipositor contains the egg canal and lumina within the valves, so we approximated it with a hollow cylinder (outer radius $15 \mu \mathrm{m}$, inner radius $10 \mu \mathrm{m}$, length $7.5 \mathrm{~mm}){ }^{6,15,43}$ Furthermore, we assumed homogeneous material properties, namely a Young's modulus of $5 \mathrm{GPa}$, which is a reasonable estimation for ovipositors and intromittent beetle organs whose Young's moduli range from $1 \mathrm{GPa}$ to $10 \mathrm{GPa} .{ }^{5,15,44}$ For simplicity of the model, we assumed that the ovipositor is fixed at both ends during the drilling phase; one end just inserted in the substrate, the other end inside the stationary abdomen. Calculations for this configuration show that buckling is likely at a load of $0.200 \mathrm{mN}$ (see SI for calculation), which is one order of magnitude smaller than the $1.20 \mathrm{mN}$ of pushing force that can be delivered by a single ventral valve and when we completely ignore any additional forces 
produced by the abdomen. However, according to the push-pull mechanisms, the

pushing force of one valve is cancelled by the pulling force on the other ones. ${ }^{6,45}$ Again, when only looking into the dynamics of the ventral valves, subtracting the pro- and retraction forces results in the net pushing force of $0.19 \mathrm{mN}$, which is close to the above calculated buckling threshold.

Furthermore, in the above calculation of the buckling threshold, we assumed that the ovipositor is unsupported along its entire length. In reality, specialized sheaths envelop the ovipositor at rest and presumably support it during the probing process. ${ }^{6,26,46}$ In D. longicaudata, the sheaths detach only when a considerable length of the ovipositor (approximately $75 \%$ ) has already been inserted into the substrate (Fig. S4). ${ }^{6}$ The sheaths increase the ovipositor width and therefore the load required for buckling. Already a modest two-fold increase of the ovipositor outer radius brings the buckling threshold to $3.955 \mathrm{mN}$, more than three times higher than what the ventral valves can deliver. This illustrates the importance of the external supporting mechanisms wasps employ during probing such as the mentioned sheaths or clamping. ${ }^{5,15,39,46}$

\section{Conclusions and future work}

The overall mechanism for ovipositor insertion of D. longicaudata shows several adaptation to overcome the challenges of slender probe insertion. The semi-circular shape of the second valvifer horn, in combination with the flexible rami of the first valvifer makes depression of the ovipositor possible, without interfering with the actuation system. Based on our estimations, the sheaths surrounding the ovipositor are essential to avoid buckling, even though pushing forces applied by the ventral valves are small. It also shows that the animals cannot apply high forces with the abdomen without the risk of buckling when part of the abdomen is still outside the substrate. The use of the push-pull mechanism is a great adaptation to overcome this. ${ }^{6,15,45}$ In some stages of the push-pull mechanism, the insertion is only done by the muscles protracting the ventral valves. Assuming the dorsal valve is under tension, then the abdomen is pulling of the ovipositor and cannot contribute to insertion. Therefore, the insertion force is mostly applied by the main actuators of ventral valve protraction (M5), which can generate a force of around $2.4 \mathrm{mN}$. This, however, appears to be more than sufficient to pierce fruits. ${ }^{43}$ Although a similar actuation system has been described in other wasp, ${ }^{\text {e.g. }}{ }^{8,11,47}$ analysis of a wider range of species, which use different probes in different substrates, will provide further insights in the subtle adaptations related to other life histories.

In addition a good understanding of the probing mechanism of wasps can be applied in the development of man-made probes with similar requirements as probing 
wasps. Thin steerable needles are an example for medical applications that can be used for hard-to-reach places in the body, and inside vulnerable tissues. A good understanding of the probing mechanism of parasitic wasps and other probing insects could therefore lead to innovations in this rapidly advancing field.

\section{Acknowledgements}

We acknowledge the Paul Scherrer Institut, Villigen, Switzerland for provision of synchrotron radiation beam time at the TOMCAT beamline X02DA of the SLS. We also acknowledge the National Institute of General Medical Sciences of the National Institutes of Health for their role in the development of the Seg3D software (grant number P41 GM103545-18) for segmentation purposes. We thank Remco Pieters for technical support; Henk Schipper for help with sample preparations; Karen LeonKloosterziel for animal care; Wouter van Veen and Cees Voesenek for IT support; Kees Spoor for providing the MatLab code for calculating muscle moment arms; Florian Link for MeVisLab support; and the user committee of the Netherlands Organization for Scientific Research Division Applied and Engineering Sciences (NWO TTW) WASP project and the members and students of the research group for their useful discussions. 


\section{References}

[1] Zен DW, Zен JA, \& Sмiтн RL (1989). Ovipositors, amnions and eggshell architecture in the diversification of terrestrial arthropods. The Quarterly Review of Biology 64, 147-168.

[2] Heatwole H, Davis DM, \& Wenner AM (1962). The behaviour of Megarhyssa, a genus of parasitic hymenopterans (Ichneumonidae: Ephialtinae). Zeitschrift fur Tierpsychologie - Journal of Comparative Ethology 19, 652-664.

[3] Elias LG, Teixeira SP, Kjellberg F, \& Santinelo Pereira RA (2012). Diversification in the use of resources by Idarnes species: bypassing functional constraints in the fig-fig wasp interaction. Biological Journal of the Linnean Society 106, 114-122.

[4] Ghara M, Kundanati L, \& Borges RM (2011). Nature's Swiss Army knives: ovipositor structure mirrors ecology in a multitrophic fig wasp community. PloS one 6, e23642.

[5] Kundanati L \& Gundiah N (2014). Biomechanics of substrate boring by fig wasps. Journal of Experimental Biology 217, 1946-1954.

[6] Cerkvenik U, Van de Straat B, Gussekloo SWS, \& van Leeuwen JL (2017). The mechanisms of ovipositor insertion and steering of a parasitic wasp. Proceedings of the National Academy of Sciences 114 (37), E7822-E7831.

[7] Godfray H (1994). Parasitoids: behavioral and evolutionary ecology. Princeton University Press.

[8] Fergusson NDM (1988). A comparative study of the structures of phylogenetic importance of female genitalia of the Cynipoidea (Hymenoptera). Systematic Entomology 13, 13-30.

[9] SNodgrass R (1933). Morphology of the insect abdomen. Part II. The genital ducts and the ovipositor. Smithsonian Miscellaneous Collections 89, 1-148.

[10] Sмiтн EL (1972). Biosystematics and morphology of Symphyta - III external genitalia of Euura (Hymenoptera: Tenthredinidae): sclerites, sensilla, musculature, development and oviposition behaviour. International Journal of Insect Morphology and Embryology 1, 321-65.

[11] SмIтH EL (1970). Evolutionary morphology of the external insect genitalia. 2. Hymenoptera. Annals of the Entomological Society of America 63, 1-27.

[12] Quicke DLJ, Fitton MG, Tunstead JR, Ingram SN, \& Gaitens PV (1994). Ovipositor structure and relationships within the Hymenoptera, with special reference to the Ichneumonoidea. Journal of Natural History 28, 635-682.

[13] Sмiтh EL (1969). Evolutionary morphology of external insect genitalia. 1. Origin and relationships to other appendages. Annals of the Entomological Society of America 62, 1051-1079.

[14] SCHUDDER GGE (1961). The comparative morphology of the insect ovipositor. Transactions of the Royal Entomological Society of London 113, 2-40.

[15] VINCENT JFV \& King MJ (1995). The mechanism of drilling by wood wasp ovipositors. Biomimetics 3, 187-201.

[16] Sakes A, Dodou D, \& Breedveld P (2015). Buckling prevention strategies in nature as inspiration for improving percutaneous instruments: a review. Bioinspiration $\&$ Biomimetics 11.

[17] Quicke DLJ, Fitton M, \& Harris J (1995). Ovipositor steering mechanisms in braconid wasps. Journal of Hymenoptera Research 4, 110-120.

[18] Quicke DLJ \& FitTon MG (1995). Ovipositor steering mechanisms in parasitic wasps of the families Gasteruptiidae and Aulacidae (Hymenoptera). Proceedings of The Royal Society: Biological Sciences 261, 98-103.

[19] Quicke DLJ (1991). Ovipositor mechanics of the braconine wasp genus Zaglyptogastra and the ichneumonid genus Pristomerus. Journal of Natural History 25, 971-977.

[20] Schudder GCE (1971). Comparative morphology of insect genitalia. Annual Review of Entomology 16, 379-406.

[21] Copland MJW \& King PE (1972). The structure of the female reproductive system in the Torymidae (Hymenoptera: Chalcidoidea). Transactions of the Royal Entomological Society of London 124, 191-212. 
22] Chapman RF (2013). The Insects: structure and function. Cambridge University Press, 5th edn.

[23] Klass KD, Matushina Na, \& Kaidel J (2012). The gonangulum: a reassessment of its morphology, homology, and phylogenetic significance. Arthropod Structure \& Development 41, 373-394.

[24] Copland MJW (1976). Female reproductive system of the Aphelinidae (Hymenoptera: Chalcidoidea). International Journal of Insect Morphology and Embryology 5, 151-166.

[25] Vilhelmsen L (2000). The ovipositor apparatus of basal Hymenoptera (Insecta): phylogenetic implications and functional morphology. Zoologica Scripta 29, 319-345.

[26] Vilhelmsen L (2003). Flexible ovipositor sheaths in parasitoid Hymenoptera (Insecta). Arthropod Structure \& Development 32, 277-287.

[27] KING PE (1962). The muscular structure of the ovipositor and its mode of function in Nasonia vitripennis (Walker) (Hymenoptera: Pteromalidae). Proceedings of Royal Entomological Society London. Series A, General Entomology 37, 121-128.

[28] Vilhelmsen L, Isidoro N, Romani R, Basibuyuk HH, \& Quicke DLJ (2001). Host location and oviposition in a basal group of parasitic wasps: the subgenual organ, ovipositor apparatus and associated structures in the Orussidae (Hymenoptera, Insecta). Zoomorphology 121, 63-84.

[29] ERnst AF, Miкó I, \& Deans AR (2013). Morphology and function of the ovipositor mechanism in Ceraphronoidea (Hymenoptera, Apocrita). Journal of Hymenoptera Research 33, 25-61.

[30] Metscher BD (2009). MicroCT for comparative morphology: simple staining methods allow highcontrast 3D imaging of diverse non-mineralized animal tissues. BMC Physiology 9.

[31] Stampanoni M, Groso A, Isenegger A, Mikuljan G, Chen Q, Bertrand A, Henein S, Betemps R, Frommherz U, Böhler P, Meister D, Lange M, \& Abela R (2006). Trends in synchrotron-based tomographic imaging: the SLS experience. Procedings of SPIE 6318, 63180M.

[32] Marone F \& StAMPANONi M (2012). Regridding reconstruction algorithm for real-time tomographic imaging. Journal of Synchrotron Radiation 19, 1029-1037.

[33] Paganin D, Mayo S, Gureyev T, Miller P, Wilkins S, Scientific C, \& Science M (2002). Simultaneous phase and amplitude extraction from a single defocused image of a homogeneous object. Journal of Microscopy 206, 33-40.

[34] CIBC (2016), Seg3D: Volumetric Image Segmentation and Visualization. Scientific Computing and Imaging Institute (SCI), Download from: http://www.seg3d.org.

[35] Evans ME (1977). Locomotion in the Coleoptera Adephaga, especially Carabidae. Journal of Zoology 181, 189-226.

[36] Goyens J, Dirckx J, Dierick M, Van Hoorebeke L, \& Aerts P (2014). Biomechanical determinants of bite force dimorphism in Cyclommatus metallifer stag beetles. Journal of Experimental Biology 217, 1065-1071.

[37] Cignoni P, Cignoni P, Callieri M, Callieri M, Corsini M, Corsini M, Dellepiane M, Dellepiane M, Ganovelli F, Ganovelli F, Ranzuglia G, \& Ranzuglia G (2008). MeshLab: an opensource mesh processing tool. Sixth Eurographics Italian Chapter Conference 129-136.

[38] Gauld ID, Bolton B, Huddleston T, Fitton MG, Shaw MR, Noyes JS, Day MC, Else GR, FerGUsson NMD, \& WARD SL (1988). The Hymenoptera. Oxford University Press, British Museum.

[39] Le LANNIC J \& NÉNON JP (1999). Functional morphology of the ovipositor in Megarhyssa atrata (Hymenoptera, Ichneumonidae) and its penetration into wood. Zoomorphology 119, 73-79.

[40] VilHeLmSEN L (2000). Before the wasp-waist: comparative anatomy and phylogenetic implications of the skeleto-musculature of the thoraco-abdominal boundary region in basal Hymenoptera (Insecta). Zoomorphology 119, 185-221.

[41] Austin AD (1983). Morphology and mechanics of the ovipositor system of Ceratobaeus Ashmead (Hymenoptera: Scelionidae) and related genera. International Journal of Insect Morphology and Embryology 12, 139-155.

[42] Quicke DLJ (2015). The braconid and ichenumonid parasitoid wasps: biology, systematics, evolution and ecology. John Wiley \& Sons, Ltd, first edn. 
[43] Leyva JL, Browning HW, \& Gilstrap FE (1991). Effect of host fruit species, size, and color on parasitization of Anastrepha ludens (Diptera: Tephritidae) by Diachasmimorpha longicaudata (Hymenoptera: Braconidae). Environmental Entomology 20, 1469-1474.

[44] Matsumura Y, Kovalev AE, \& Gorb SN (2017). Penetration mechanics of a beetle intromittent organ with bending stiffness gradient and a soft tip. Science Advances 3, eaao5469.

[45] Cerkvenik U, Dodou D, van Leeuwen JL, \& Gussekloo SWS (2018). Functional principles of steerable multi-element probes in insects. Biological Reviews .

[46] Vilhelmsen L \& TuRrisi GF (2011). Per arborem ad astra: morphological adaptations to exploiting the woody habitat in the early evolution of Hymenoptera. Arthropod Structure \& Development 40, $2-20$.

[47] Whiting AR (1967). The biology of the parasitic wasp Mormoniella vitripennis (= Nasonia Brevicornis) (Walker). The Quarterly Review of Biology 42, 334-406. 


\section{Supporting information}

\section{SI Size and staining correction of the muscle cross-sectional area and moment arms}

As described in the main document, maximal muscle forces were estimate on the basis of the physiological cross-sectional area (PSCA) of the muscles. Because freezing and/or staining might affect the PCSA we used a freshly decapitated animal as a control to correct for these effects. Muscle forces were estimated in two animals, one in probing position and one in resting position. The estimates of both these animals were scaled to the size of the control animal to correct for size differences.

For the correction, we assumed that the exoskeleton would not be affected by freezing and or staining, so we used two measures on the 2 nd valvifers to estimate the linear correction coefficient. We measured the distance between the frontal bulge and the articulation with the first valvifer (h1, sagittal plane), and the internal distance between the left and right articulation h1 (transverse plane) in the reconstructed polygon meshes. Each measure was taken four times and then averaged. Dividing the average of each measurement of each stained specimen by the corresponding average of the unstained specimen gave us two scaling factors for each specimen. These two were averaged to obtain the final linear scaling factor $\left(l_{\mathrm{sf}}\right)$. Assuming isometric scaling, this resulted in an area scaling factor $\left(a_{\mathrm{sf}}\right)$ equal to $l_{\mathrm{sf}}^{2}$ (Table S1).

Using the area scaling factor, we estimated for each specimen the expected CSA based on isometric scaling. The ratio between the predicted PCSA and the measured PSCA is the treatment correction factor $\left(t_{\mathrm{cf}}\right)$ for freezing and staining effects (Table S2). The total correction for the measured PSCA is therefore:

$$
\mathrm{PCSA}_{\text {actual }}=\mathrm{PCSA}_{\text {measured }} \cdot l_{\mathrm{sf}}^{2} \cdot t_{\mathrm{cf}} \text {. }
$$


Table S1: Determination of size correction factors.

\begin{tabular}{cccccc}
\hline plane & $\begin{array}{c}\text { distance } \\
(\mu \mathrm{m}, n=4)\end{array}$ & $\begin{array}{c}\text { ratio } \\
\text { unstained } \\
\text { stained }\end{array}$ & $\begin{array}{c}\text { linear scaling } \\
\text { factor }\left(l_{\mathrm{sf}}\right)\end{array}$ & $\begin{array}{c}\text { area scaling } \\
\text { factor }\left(l_{\mathrm{sf}}^{2}\right)\end{array}$ \\
\hline Unstained & $\begin{array}{c}\text { sagittal } \\
\text { transverse }\end{array}$ & $\begin{array}{c}255.399 \\
167.541\end{array}$ & 1 & 1 & - \\
$\begin{array}{c}\text { Resting } \\
\text { IKI stained }\end{array}$ & sagittal & 230.125 & 1.110 & 1.218 & 1.483 \\
$\begin{array}{c}\text { Probing } \\
\mathrm{I}_{2} \text { E stained }\end{array}$ & sagittal & 277.923 & 0.919 & & \\
\hline
\end{tabular}

Table S2: Determination of treatment correction factor.

\begin{tabular}{cccccc}
\hline Muscle & $\begin{array}{c}\text { Mean cross- } \\
\text { sectional area } \\
\left(\mu \mathrm{m}^{2}, n=5\right)\end{array}$ & $\begin{array}{c}\text { Size corrected } \\
\text { cross-sectional } \\
\text { area }\left(\mu \mathrm{m}^{2}\right)\end{array}$ & $\begin{array}{c}\text { ratio } \\
\text { unstained }\end{array}$ & $\begin{array}{c}\text { Treatment } \\
\text { stained }\end{array}$ & $\begin{array}{c}\text { Trection factor } \\
\left(t_{\mathrm{cf}}\right)\end{array}$ \\
\hline Unstained & large & 2511.048 & - & 1 & 1 \\
sesting & small & 314.765 & - & 1 & 1 \\
IKI stained & large & 1929.287 & 2861.070 & 0.878 & 0.845 \\
Probing & small & $261.499^{+}$ & 387.795 & 0.812 & \\
$\mathrm{I}_{2}$ E stained & large & 1298.570 & 1201.827 & 2.089 & 1.932 \\
\hline
\end{tabular}

${ }^{\dagger}$ based on ratio small/large in other specimens

\section{SI Critical buckling load}

The buckling threshold of a beam can be calculated using the Euler-Bernoulli equation:

$$
P_{\mathrm{cr}}=\frac{\pi^{2} E I}{(K L)^{2}}
$$

where $E$ is the Young's modulus of the beam (assuming homogeneous material properties), I its second moment of area, which accounts for the mass distribution around the bending axes of the beam, $K$ is the effective length factor of the cylinder accounting for its end conditions, and $L$ is the unsupported length of the beam.

We estimated that the insect cuticle has the Young's modulus of $5 \mathrm{GPa}$, which is reasonable, based on the estimation of the stiffness of similar structures in other insects, namely other ovipositors or intromittent organs of beetles $(5,14,45)$. Because we are interested in the situation after the ovipositor punctures the substrate (and when the push-pull mechanism can be employed), we assumed both ends of the 
ovipositor are fixed $(K=0.5)$; one in the substrate, the other one in the abdomen of the animal. We approximated the ovipositor of $D$. longicaudata as a hollow cylinder of similar dimensions, which has also been done previously for ovipositors of other species (14). The values of the outer $\left(r_{\text {out }}=15 \mu \mathrm{m}\right)$ and inner radius $\left(r_{\text {in }}=10 \mu \mathrm{m}\right)$ of the cylinder were estimated from the cross-sections of the valves, while the length of the ovipositor was taken as $5.7 \mathrm{~mm}$ based on (44). The second moment of area of a hollow cylinder can be calculated with (49):

$$
I=\frac{\pi}{4}\left(r_{\text {out }}^{4}-r_{\text {in }}^{4}\right) .
$$

The effect of the sheaths was estimated using $r_{\text {out }}=30 \mu \mathrm{m}$.

\section{SI Determining valve offset}

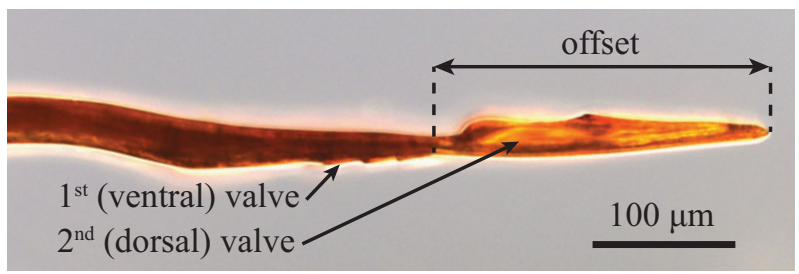

Fig. S1. Micrograph of the ovipositor with the retracted first valve configuration. This was the only valve configuration obtained naturally by freezing the probing wasps. Other configurations were obtained by manually adjusting the valves after thawing.

The offset of the retracted ventral valves $\left(o_{\text {prot }}\right)$ was measured from a micrograph image of the ovipositor ventral valves (Fig. S1). Other configurations were obtained by manually sliding the valves along each other after thawing of the specimens, using tweezers. This caused the valves to separate at their tips, which made it impossible to determine the offset using a microscope. Instead, we calculated the offset $\left(o_{\text {prot }}\right)$ by assuming that it scales linearly with the rotation of the first valvifer and with the size of the animal, but that the latter does not affect the angles of first valvifer movements (i.e. isometric scaling):

$$
\frac{r_{\text {ret }}}{r_{\text {prot }}} \frac{o_{\text {prot }}}{\Delta \alpha_{\text {prot }}}=\frac{o_{\text {ret }}}{\Delta \alpha_{\text {ret }}}
$$

where $r_{\text {prot }}$ and $r_{\text {ret }}$ are the heights of the valvifers in the pro- and retracted configuration (scaling factor), and $\Delta \alpha_{\text {prot }}$ and $\Delta \alpha_{\text {ret }}$ are the angles of the first valvifer with respect to the reference configuration with aligned valves (offset $0 \mu \mathrm{m}$, angle $\alpha_{\text {ref, }}$, see Table 3). These were calculated using: 


$$
\Delta \alpha_{i}=\left|\alpha_{i}-\alpha_{\text {ref }}\right|,
$$

where index $i$ stands for either pro- or retraction.

To confirm that the manual adjustments of the valves correlate with the movements of the first valvifer, we compared the valvuale offset with the excursion of the dorsal point of the first valvifer during its rotation (i.e. the arc length; $s_{\text {prot }}, r_{\text {ret }}$ ). The arc lengths were calculated with respect to the same reference position of aligned valves as above (s $0 \mu \mathrm{m}$, angle $\alpha_{\text {ref }}$ ):

$$
s_{i}=\frac{\pi}{180} r_{i} \Delta \alpha_{\text {prot }}
$$

where the $r_{i}$ is the height of the first valvifer (see Table 3) in protracted or retracted configuration.

\section{SI Histology of the ovipositor base}

For histological sectioning, the abdomen with the intact ovipositor was dissected from the body and fixed with a mixture of $0.1 \mathrm{M}$ sodium cacodylate buffer (dimethylarsenic acid sodium salt trihydrate; Merck, Germany), 2\% (weight/volume) paraformaldehyde (Merck, Germany), and 2.5\% (volume/volume) glutaraldehyde (EMS, PA, USA) on ice $\left(0^{\circ} \mathrm{C}\right)$ for $2.5 \mathrm{~h}$. The sample was washed with the $0.1 \mathrm{M}$ cacodylate buffer twice at $0^{\circ} \mathrm{C}$, dehydrated in a series of ethanol solutions of increasing concentration from $50 \%$ to $100 \%$, and transferred to epoxy resin (epon; Embed-812 Embedding Kit, EMS, PA, USA) through a sequence of solutions: a mixture of ethanol and propylene oxide (PO), pure $\mathrm{PO}$, a series of $3: 1$ and $1: 1$ mixtures of $\mathrm{PO}$ and epon, and finally pure epon. The samples in epon were put in moulds and left to harden overnight at $60^{\circ} \mathrm{C}-65^{\circ} \mathrm{C}$.

Semi-thin sections of $1 \mu \mathrm{m}$ and $2 \mu \mathrm{m}$ thickness were cut using a Reichert Ultracut $\mathrm{S}$ ultramicrotome (Leica Microsystems $\mathrm{GmbH}$, Germany) and stained with a mixture of 1\% Toluidine blue (VWR, PA, USA) and 1\% Borax (disodium tetraborate decahydrate; Merck, Germany) dissolved in distilled water. The sections were photographed with a Leica DFC 450 C camera (5 MP) mounted on a Leica DM6b microscope (Leica Microsystems) with $40 \times$ or $100 \times$ objective. The images were stored as tiff files. 

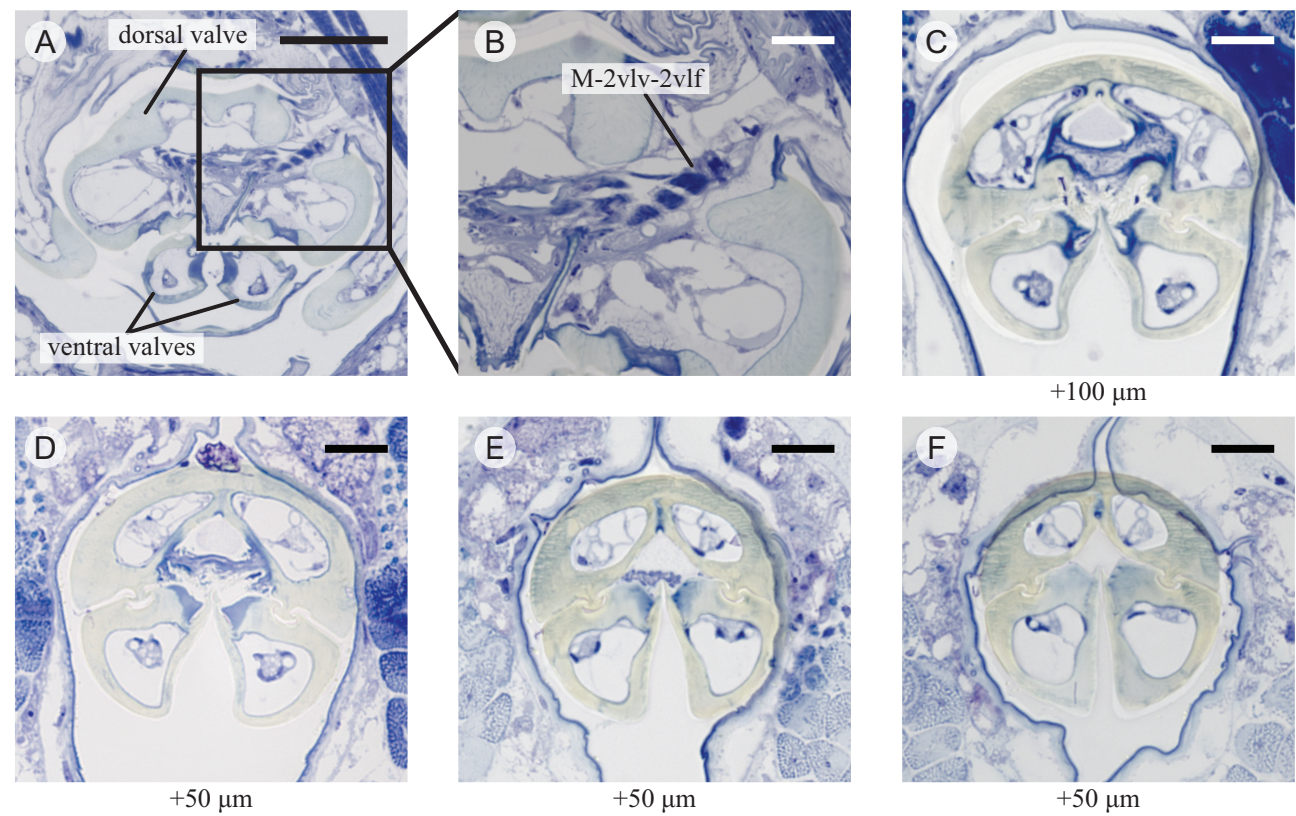

Fig. S2. Cross-sections along the ovipositor base from anterior (A) to posterior (F). The dorsal valve is positioned on top, the two ventral valves on the bottom. (A, B) Section through the widened base of the ovipositor showing the striation in one of the muscle bellies of M-2vlv-2vlf (B). (C-F) Cross-sections through the ovipositor show the M-2vlv-2vlf attaches into the medial walls of the dorsal valve (stained blue), but does not reach deep into the ovipositor, which is generally more than $5 \mathrm{~mm}$ long. ${ }^{43}$ Scale bars: $50 \mu \mathrm{m}(\mathrm{A}), 10 \mu \mathrm{m}$ (all the rest).
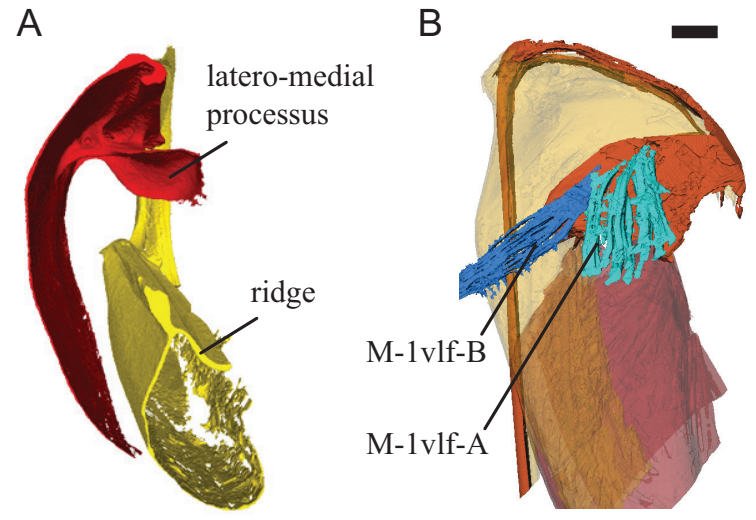

Fig. S3. Details of muscle attachment sites (A) and muscles attaching to the first valvifer (B). (A) Posterior view of the anterior part of the left T9 and second valvifer showing the attachment sites of M6. (B) Two muscles attach to the first valvifer, but we did not investigate their insertions. M-1vlf-A is directed posteriorly, while the M-1vlf-B attaches ventral to the ovipositor apparatus. Scale bar: $50 \mu \mathrm{m}$ (B). 


\section{SI Sheath detachment}

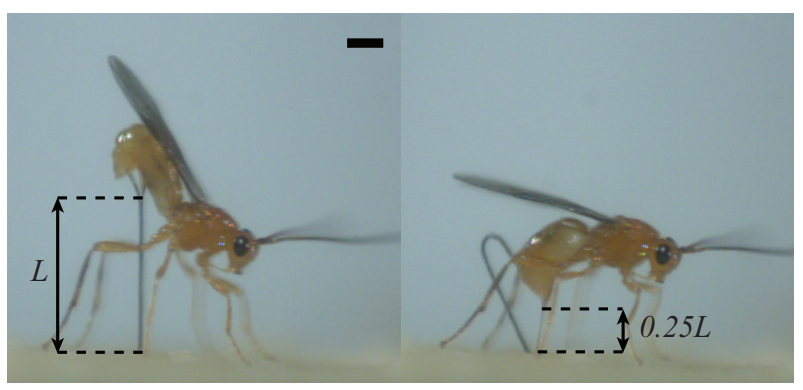

Fig. S4. The length of the ovipositor at the time of sheath detachment. These are still images from a movie of a probing $D$. longicaudata. Left: the wasp at the onset of probing, just before puncturing the substrate. Right: the moment of complete sheath detachment. Scale bar: $1 \mathrm{~mm}$. 
<smiles>C=CC#CC(=C)CC</smiles> 
chapter

General discussion 



\section{Introduction}

Insects often probe for resources that are hidden within substrates. ${ }^{1-3}$ Understanding the probing mechanisms in insects can bring insight in their ecological interactions and evolution, and can also help in the development of man-made probes. Insect probes are complex and evolved through the process of natural selection by satisfying a number of functional and behavioural requirements, while being limited by phylogenetic constraints that differ across taxa. Insects often target resources hidden deep within the substrates, which requires long and thin (slender)

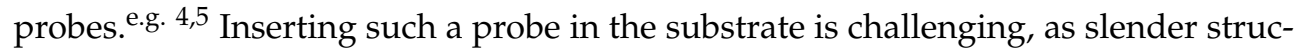
tures easily bend and break (buckle) when axial load is applied. Probes, however, appear not to get damaged during probing and are used repeatedly throughout the lifetime of individual insects. ${ }^{6-8}$ Interestingly, probes used in solid substrates consist of multiple sliding elements, indicating that this is an adaptation that might avoid damage to the probe. ${ }^{2,9,10}$ Furthermore, as explained in the introduction, it is beneficial that probes are capable of changing their direction during probing. The functional requirements of buckling avoidance and steering are not only interesting from a biological perspective, but also for developing novel, minimally invasive medical tools, such as slender and steerable needles. e.g. 11,12 In this thesis, I therefore focused on how insects use their slender probes for exploration of solid substrates.

Many insects including butterflies, mosquitoes and flies, hemipterans, and parasitic wasps evolved probes for searching and securing food from within the substrates. Examples include nectar at the end of elongated flowers, ${ }^{13}$ blood coursing through the vessels under animal skin, ${ }^{2}$ phloem or xylem sap flowing through plants, ${ }^{14}$ or animals living in enclosed spaces such as leaf rolls, galls, and wood. ${ }^{6,15}$ The probes are used to determine the location of the resource, asses its quality, and ingest the food or deliver eggs to the food, which will be ingested by the developing offspring. ${ }^{1,7,16}$

Lepidopteran and fly mouthparts that are used for piercing fruits or animal skin are generally short, robust and, in the case of lepidopterans, also contain intrinsic musculature. ${ }^{17-19}$ In contrast, the probes of mosquitoes, hemipterans, and parasitic wasps are often very slender and driven by musculature located at their base. ${ }^{8,20} \mathrm{I}$ therefore focused in this thesis on the slender probes of mosquitoes, hemipterans, and parasitic wasps. The selection pressures driving the evolution of probes towards high slenderness are not entirely clear, but presumably (i) less energy (work) is needed to insert a thin structure compared to a wide one inside the same substrate, (ii) slender probes inflict less damage to the substrate or the host which 
increases both the survival of the probing animal (e.g. mosquitoes risk to be swatted if they are not stealthy) and that of the host (larvae of parasitic wasps often feed from live hosts), and (iii) slender probes facilitate substrate exploration because they are more flexible and can be more easily steered in the substrate compared to wider probes of the same material.

Below, I place our findings on probing of Diachasmimorpha longicaudata (Braconidae) in an integrative analysis of probing with slender structures, discuss the ecological relevance of probing, and indicate how the findings can contribute to the development of man-made probes.

\section{Biomechanics of probing with slender structures}

The act of probing in a substrate can be divided into three distinct phases: puncturing, deeper insertion, and retraction of the probe. The first and the last phase will be discussed only briefly as this thesis is mostly focused on the second, explorative phase of probing.

\section{Puncturing the substrate}

Puncturing the substrate is done by pushing the tip of the probe into the substrate. Axial loading of the probe cannot be avoided which makes this a high risk phase in terms of buckling. Wasps with long ovipositors such as our model species D. longicaudata, lift their abdomen and ventrally deflect the ovipositor at its base, before puncturing the substrate ${ }^{5,21}$ (chapter 3). In mosquitoes, wasps, and hemipterans (excessive) buckling of the probe is most commonly avoided by providing the probe with lateral support (chapter 2). This is particularly useful as such support can both reduce the effective free length of the probe and increase its effective width, which increases the critical axial load that causes buckling of a probe. Support is generally achieved with sheaths that envelop the probe. ${ }^{1,22,23}$ The sheath is in mosquitoes and hemipterans a single modified, gutter-like mouthpart element, the labium, ${ }^{1,24}$ while wasps, including $D$. longicaudata, generally possess a pair of sheath appendages. ${ }^{25}$ How much lateral support is provided by the sheaths is not completely clear, because they are flexible and detach from the probe during its insertion $1,10,25,26$ (chapter 3). However, at least in mosquitoes and wasps, the detachment of sheaths occurs only after a considerable length of the probe has been inserted ${ }^{24,27}$ (chapter 3). It is unclear whether the sheaths of hemipterans also follow the same dynamics. I hypothesize that the sheaths offer support during puncturing as well as during deeper insertion despite their flexibility. Presumably, the sheath detachment occurs when the length of the probe outside the substrate is short enough to sustain axial loading. This hypothesis can be tested by investigating 
the material and structural properties of both the probe and sheath(s), that should be coupled to the measurement of the external probe length at the time of sheath detachment. The evolution of slender probes also requires the (co)evolution of the supporting structures that provide the probes with lateral support and prevent buckling. Little is known about these processes and more work is needed in how the (shape) changes in the probes relate to (shape) changes of the support.

\section{Further insertion}

After successful insertion of the probe tip inside the substrate, the second, explorative phase can begin. To optimally explore the substrate from a single puncture point, it is beneficial to be able to reach a considerable depth and lateral range during probing (chapter 3). Depth is achieved by inserting the probe perpendicular to the substrate surface, while the lateral range can be widened by controlling the deviation of the probe from such a perpendicular path. Two force directions are therefore important: along the longitudinal probe axis (insertion) and normal to the probe (lateral deviation).

\section{Buckling avoidance}

The main forces relevant for probing acting in the longitudinal axis of the probe are the insertion force, friction along the probe, and the cutting forces at the probe tip. As explained in chapter 2, these forces depend on the forces exerted by the probing animal, substrate properties (stiffness, toughness), probe geometry, and depth of probe insertion. When pushing the probe inside the substrate, the friction force increases with the needle diameter and insertion depth for any given substrate. ${ }^{28-30}$ The increased friction requires higher pushing forces, which might be an issue for long, slender probes as these forces could lead to buckling of the non-inserted parts despite the avoidance measures described above and in chapter 2 . The ovipositor could, for example, disengage from the lateral supports, or the supports themselves might buckle (e.g. flexible sheaths).

One of the arguments in this thesis is that the probes consisting of multiple, longitudinally interconnected, and sliding elements evolved independently in disparate insect taxa as a response to buckling avoidance and the need to steer the probe in a desired direction spatial steering demands ${ }^{10,31,32}$ (chapter 2). Independent movements of the elements, which are termed valves in wasps, ${ }^{9,33}$ reduce the risk of buckling as explained by the push-pull mechanism ${ }^{34}$ (chapters 2,3 ). This mechanism relies on the difference between the mean pushing and pulling (anchoring) forces within a probing cycle-the average anchoring forces need to be higher than the average pushing forces. This might be reflected in the arrangement and size of the 
probing musculature with pulling muscles generating greater forces on the valves than the pushing muscles. Indeed, our estimates of the forces driving the ventral valves showed that pulling forces are higher than the pushing forces (chapter 5). The ovipositor actuation muscles presumably reflect the forces required to probe in the preferred substrates. In stiff substrates, the cutting forces are higher than in soft ones. The wood probing wasps such as the phytophagous Symphyta ${ }^{15}$ or the parasitoids such as Megarhyssa atrata ${ }^{5}$ or Rhyssa persuasoria ${ }^{35}$ need sufficiently high forces to penetrate this tough material, which might result in large muscles relative to their body than observed D. longicaudata which probes in soft fruits ${ }^{36}$ (chapter 5).

Interestingly, the investigation of the basal elements of the ovipositor and the associated musculature in D. longicaudata showed that the individual valves do not move completely independently as hypothesized in chapter 2. Rather, it appears that the ventral valves can freely slide along the dorsal valve, whereas the dorsal valve is moved only due to movements of the whole abdomen (chapter 5). I hypothesize that recorded dorsal valve protraction (chapter 3 ) is the result of simultaneous pushing of the abdomen towards the substrate and retraction of the ventral valve(s), which keeps these valves stationary in the substrate. A similar system seems to also be present in mosquitoes whose maxillae and mandibles slide along a the labrum that is only moved together with the head. ${ }^{37,38}$

The difference in pushing and pulling forces can be amplified with ovipositor valves that experience low friction when protracted in the substrate and high friction when retracted. This directional friction is achieved with enlargements or harpoon like serrations on the distal parts of the valves, which is commonly observed across the probing insects. ${ }^{34,39-42}$ Despite phylogenetic constraints, the sclerotization (stiffness), size, and number of probe serrations can be linked to specific substrate properties. Ovipositors used in stiff substrates are more sclerotized (stiffer) than those used in soft substrates. ${ }^{39,43,44}$ Furthermore, ovipositors used in fibrous substrates such as wood either have few large tip serrations or more smaller ones. ${ }^{45,46}$ Both are presumably adaptations to increase the friction between the ovipositor and the wood, as high forces are needed to tear the wood fibres during reciprocal valve movements. ${ }^{34}$

Friction due to large serrations might, however, also hinder the wasp oviposition behaviour. Many parasitic wasps lay eggs inside (endoporasitoids) their hosts, which often continue growing, thus providing food for the wasp larva. ${ }^{47,48}$ If these hosts live in stiff substrate, the wasps might need to balance the requirements of increased friction (large serrations) and inflicting little damage (small serrations) to the host. The wasps might have adapted to this challenge by laying eggs near the hosts (ec- 
toparasitoids) ${ }^{49}$ or by attacking sufficiently nutritious hosts that die upon laying of the egg. ${ }^{50-52}$ Additionally, big serrations might hinder ovipositor extraction from the substrate. In extreme cases observed some social hymenopterans, the stinger, which is a highly modified ovipositor, gets so firmly lodged in the substrate it is impossible to retract it, causing tearing of the insect's abdomen. ${ }^{53,54}$

To what extent the substrate affects the evolution (and development) of the probes is currently hard to determine, as the number of studies quantifying the probe properties relevant for insertion such as stiffness, toughness, and (non)homogeneity, is currently low. Even when such measurements were taken, similar quantification has, to my knowledge, not been extended to the substrates these probes are used in.

The push-pull mechanism has hitherto not been experimentally tested in living animals, although rough observations of probe movements in the substrates have been made. ${ }^{42,55}$ In chapter 3 , we show that the parasitic wasps D. longicaudata insert their ovipositors either using alternating valve movements, or by simply pushing all valves in the substrate at the same time. Pushing was observed in soft substrates, while reciprocal movements where the valves are pushed individually were always used in stiff substrates. This is presumably because higher forces are needed to penetrate (cut through) stiff substrates compared to soft ones. The high forces increase the risk of buckling, which is avoided using the alternating valve movements during insertion.

The speed of ovipositor insertion was also substrate dependent and decreased with increasing stiffness, presumably because the animals used reciprocal valve motions during probing (chapter 3). The decline in speed can be partly attributed to increased substrate resistance, but it also depends on the kinematics of valve movements. Frequency and amplitude of valve movements are likely the most important parameters affecting the insertion speed. Assuming no slippage between the valves and the substrate, then higher frequency or amplitudes lead to faster insertions. However, if slippage is present, a high frequency of valve motions (i.e. speed of the valve movements) might hinder insertions. Some slippage was observed during ovipositor insertions and I hypothesize, that in a given substrate, there is an optimum set of frequency and amplitude of valve movements that leads to fastest insertion speeds. The optimal parameter(s) presumably depend at least on the friction between the valves and the substrate and on the energy needed to induce fractures (cutting forces) in the substrate. It would be interesting to quantify the frequency and amplitude of valve motions during ovipositor insertions in different substrates. Comparing such values to a theoretical model of probing, could reveal whether or not animals optimize their probing behaviour to the substrate (e.g. max- 
imize energy efficiency) or speed of insertion. Slow speeds lead to lengthy probing times-usually several minutes, but up to hours in wood probing parasitoids. ${ }^{6,56}$ During probing insects are stationary and exposed to predators. Therefore, they might be adapted for high insertion speeds, rather than energy efficiency. Additionally, analysing detailed valve kinematics in different substrates might also give insights in whether wasps recognize the stiffness of the substrate, decide which insertion strategy, pushing the whole ovipositor or moving valves independently, they will use, or determine to stop probing if the substrate is beyond their capabilities.

BUCKLING-AVOIDANCE IN SLENDER MAN-MADE PROBES. The push-pull mechanisms is presumably an adaptation for avoiding high net pushing forces, which reduces the risk of damage to the probe. Implementing such a mechanism into a medical needle can therefore lead to minimization of the needle diameter, which would reduce the damage to the patient during surgery. At least two multi-element needle prototypes inspired by wasp ovipositors are currently under development, one at Imperial College London (IC London), and another at the Delft University of Technology (TU Delft). ${ }^{57-61}$ The two prototypes differ in the shape and number of individual elements (up to four at IC London, and seven at TU Delft), minimal needle diameters (to my knowledge $2.5 \mathrm{~mm}$ at IC London, ${ }^{62} 1.2 \mathrm{~mm}$ at TU Delft), and the type of inter-element connections (tongue-and-groove at IC London, a series of rings at TU Delft). In both prototypes, the limiting factor for reaching small diameters is the mechanism holding the elements together. By implementing an essentially different mechanism for connecting the elements than what is observed in wasps, the TU Delft prototype can be made thinner than the IC London prototype. This indicates the importance of incorporating into practice the principles and not necessarily the exact geometries of the biological systems. Both prototypes are flexible and can buckle if pushed inside the substrate. Instead, the push-pull mechanisms is used to generate net pulling forces and the needle can self-propel into the substrate. ${ }^{57,63}$

\section{Steering of the ovipositor}

The steering capabilities of many insect probers are not clear, but I expect that probe steering correlates with at least three parameters: host type (moving/stationary), host location (strongly concealed/exposed), and properties of the substrate (soft/stiff, complex/homogeneous). Steering is presumably not necessary when wasps attack easily accessible hosts or large targets as in the case of phytophagous species. These phytophagous wasps lay eggs in plant tissues or induce galls and have no need for accurate placement of their eggs. Instead, the wasps create a suitable microenviron- 
ment for their larvae by injecting fluids or symbiotic fungi, which change the growth or composition of the plant tissue. ${ }^{64-66}$ Nevertheless, some degree of steering has been reported for Sirex noctilio, which lays eggs in tree trunks. ${ }^{66-68}$ This is presumably because healthy trees are resistant to fungal disease spread by the wasps and it may be beneficial for the wasp to spread the fungi over a large area within the tree. Ovipositor steering might therefore increase the chances of successful inoculation of trees.

In contrast to phytophagous wasps, parasitic wasps need to accurately place their eggs inside the substrate and can greatly benefit from guiding their ovipositors during probing. This may be particularly important in species targeting hosts that are moving or hiding in complex substrates that contain stiff regions, which might hinder ovipositor insertion ${ }^{69}$ (chapter 3 ). The complex probing trajectories of wasp parasitoids suggest that properties of both the substrates and the hosts affect the evolved wasp steering capabilities ${ }^{70,71}$ (chapter 3). However, current data is limited to only a few wasp species. More studies that compare the probing of wasps adapted to explore starkly different natural substrates are needed to substantiate the correlation between steering capabilities of wasps and their host and substrate preferences.

The steering of the multi-element probe can be based on the interactions between its elements, on interactions between the elements and the substrate, or a combination of both. The substrate interactions cause bending of the probe, when the forces acting on its tip during insertion are asymmetric. Such forces are usually the result of an asymmetric tip geometry (i.e. bevel), as shown in manmade needles. ${ }^{11,72}$ The tips of insect probes are generally asymmetric ${ }^{21,39,41,73,74}$ and will therefore generate a curved insertion trajectory in the substrate. Bending of the probe is dependent on the stiffness of both the probe and the substrate. For example, a stiff probe inserted in a soft substrate might not bend, despite the asymmetric tip forces, if these cannot induce sufficient bending moments in the probe. Using the same probe in stiffer substrates, would lead to higher probe curvatures during insertion. The amount of curving can also be controlled by controlling the asymmetry of the probe tip, which makes probing less dependent on the properties of the substrate.

The reciprocal movements of the valves inherently cause overall shape changes of the asymmetric ovipositor tip due to the offset of the valves (chapters 2, 3). The recorded valve offset in D. longicaudata is very small (only around $200 \mu \mathrm{m}$ ) and, if additional mechanisms would be absent, the resulting shape change of the tip might not be sufficient to induce strong bending within the substrate. At least three mechanisms that enhance the asymmetry of the probe tip evolved in insects. These mechanisms induce bending by relying on anatomical and material differ- 
ences between the probe and can be summarized as follows. In the differential sclerotization mechanism, bending is achieved by aligning stiff and soft element regions next to each other. ${ }^{75}$ In the mechanism of 'preapical stops', the element offset causes compression and tensile forces in different probe elements that consequentially bend. ${ }^{76}$ In the mechanism of 'pre-curved' elements, the element tips are inherently curved (i.e. curved in the unloaded situation), but are straightened when the elements are aligned. ${ }^{32}$ When such elements are protracted, they assume their natural curved shape. The mentioned mechanisms rely on individual movements of the elements and controlling them implies that the animal can control the probing direction.

As observed for D. longicaudata, the ovipositor outside the substrate curves dorsally when the valves are offset (chapter 4). This is not the case inside the substrate, where the ovipositor only curves dorsally when the ventral valves are protracted and not when the dorsal valve is protracted (chapter 3). In chapter 4, we showed that curving of the ovipositor relies mostly on the interactions between the valves, although interactions with the substrate probably also play a role-they seem to enhance dorsal curving of the ventral valve, while opposing dorsal curving in the dorsal valve. The substrate can therefore still strongly influence the curving of the probe. For example, the shape changes of the probe tip might be reduced in very stiff substrates, which could decrease the steering of the probe. The above described bending mechanism in D. longicaudata only partly explains the probing capabilities of this wasp. During probing, the azimuth of the wasp's body does not significantly change and curving of the ovipositor in only one (dorsal) direction cannot explain how the animals are able to probe in any direction with respect to their body orientation (chapter 3).

How the wasps change the direction of bending up to $\sim 180^{\circ}$ during probing, sometimes in a single insertion, is still unclear. One possible explanation is that wasps rotate their entire ovipositor around its longitudinal axis. This has been suggested for both hymenopteran ovipositors and stingers, ${ }^{77,78}$ as well as hemipteran mouthparts. ${ }^{74}$ However, our own anatomical analysis of the ovipositor actuation unit suggests that rotations at the base are limited to a couple of degrees at most (chapter 5) and cannot explain the observed probing capabilities of D. longicaudata. Another possible explanation is that the wasp induces twisting of its ovipositor, which may be possible if changing the ventral valve offset induces torsional moments within the ovipositor. During our kinematics study (chapter 3), we at times observed what appeared like twisting or rotation of the ovipositor as indicated by the relative locations of the dorsal and ventral valves during probing. For example, the dorsal valve appeared on different sides at the beginning and towards the end of insertion 
(Fig. S5). The rotation or twisting of the ovipositor are currently the only working hypotheses that could explain the probing range of D. longicaudata. I expect that a future in-depth mechanical analysis could produce fascinating results, which will add to the knowledge of probing insects and might be adapted for man-made probes.
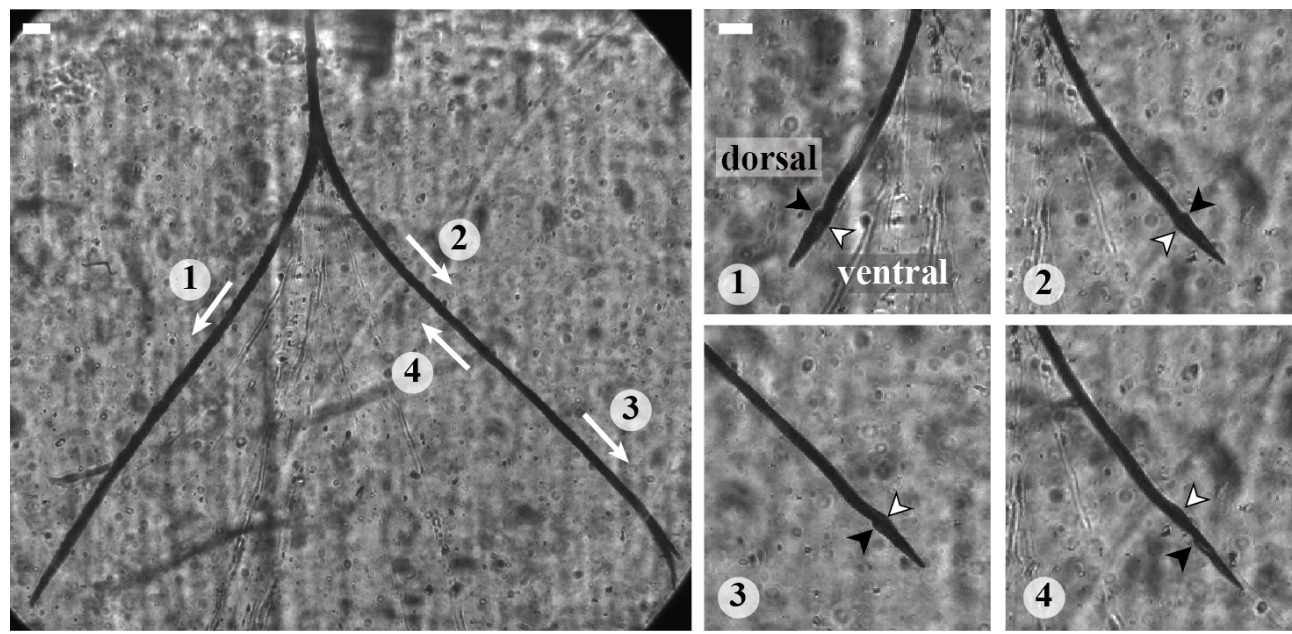

Fig. S5. Two ovipositor insertions of D. longicaudata in a soft substrate. Left image is the minimum intensity overview of both insertions. Numbers denote sequence of probing and the approximate locations of the details shown on the right. Arrows denote the movement of the ovipositor in the image on the right, while arrowheads denote the bulge of the dorsal valve (black) and slight enlargement of the ventral valves (white). The dorsal valve is on top in both (1) and (2), despite these being inserted in opposite directions. Furthermore, the dorsal valve appears on the bottom of the ovipositor in (3), which is part of the same insertion as (2), and presumably allowed the wasp to make a downwards curved trajectory before retracting the ovipositor (4). In (4) the valve offset (retracted ventral valves) is clearly visible. Scale bars: $100 \mu \mathrm{m}$.

Ovipositor characteristics reflect the phylogenetic origin of the animals, their reproductive strategy, and presumably also their preferred probing substrate. However, studies linking the morphology of the ovipositors with the substrates are few. It appears that insects exploit both the interactions with the substrate and the asymmetry-enhancing mechanisms to control the direction of probing, which might be important for the understanding of the wasp-host interactions. A reduced probing range in stiff substrates might, for example, lead to a smaller number of laid eggs (and lower/reduced fitness) compared the situation where wasps probe in soft substrates. Knowledge on probing capabilities can therefore bring insights into the life-cycle of the parasitoids and their ecological roles.

Steering of MAN-MAde Probes. The above described IC London and TU Delft needle prototypes markedly differ in their steering capabilities. To compare their curving capability with that of D. longicaudata, I converted the maximal reported 
curvature of the prototypes to dimensionless curvature. As in chapter 3, this was done by multiplying the curvature with the diameter of the curved structure. To my knowledge, the highest dimensionless curvatures can be obtained with the IC London prototype measuring $4 \mathrm{~mm}$ in diameter, which can be steered only in one plane $(0.057),{ }^{60}$ whereas the prototype of the same diameter that can be steered in $3 \mathrm{D}$ achieved a slightly lower dimensionless curvature (around 0.45). ${ }^{61}$ These values are comparable to the maximal dimensionless curvature of the ovipositor of D. longicaudata (0.048, chapter 3), but are markedly higher than that of the TU Delft prototype (0.002). ${ }^{57}$ These differences can presumably at least in part due to the different material properties of the probes and the substrates. The IC London prototype was 3D printed using two materials of different Young's moduli ( $E$; $0.05 \mathrm{GPa}$ and $0.006 \mathrm{MPa}$ ) and tested in $6 \%$ gelatine with $E=7 \mathrm{kPa} .{ }^{60}$ The TU Delft prototype consists of metal (Nickel Titanium) wires and was tested in $4 \%$ gelatine $(E=4.65 \mathrm{kPa})$, while the wasps probed with their ovipositor $\left(E_{\mathrm{eff}} \approx 3.6 \mathrm{GPa}\right.$; chapter 4$)$ in $4 \%$ phytagel ( $E=0.2 \mathrm{GPa}$; chapter 3$)$. Additionally, the variation in the probe curving capabilities may also be partly due to differences in probe element interconnections. These are strong tongue-and-groove mechanism in the IC London prototype and wasp ovipositor, while the elements of the TU Delft prototype are held together only at certain locations along their length. Any change in the direction of an individual element is therefore more likely to be transferred to the other elements in the IC London prototype and the wasp ovipositor, than in the TU Delft prototype. For a medical applications, it is important that the needle is both thin and can attain high curvatures. The IC London prototypes for which high curvatures are reported are still quite thick and might have limited use. The TU Delft prototype, is very thin, but currently cannot achieve high curvatures.

Curving of the needle prototypes could be improved by bevel enhancement mechanism as observed in insects. This has been partially done in the TU Delft prototype by adjusting the shape of the most distal holder that keeps the needle elements together. The holder was made conical and, depending on its orientation, either caused the elements to converge or diverge. ${ }^{79}$ Element offset using a conical holder led to more pronounced bevels and improved steering when compared to the prototype with a parallel holder, although the curvatures achieved with the conical holder are not reported. ${ }^{57,79}$

Next to bevel enhancement mechanisms, some insect probes also possess a soft region that might facilitate bending near their distal ends (chapter 4). A hinge in the distal end of the needle has been implemented in a non-bioinspired needle, which resulted in enhanced bending during insertion in the substrate. ${ }^{80}$ Control of curvature can be achieved by rotating the needle around its longitudinal axis during 
insertion. ${ }^{81}$ Such needle rotations might not be suitable when complex trajectories inside the tissue are needed, although this is probably dependent on the tissue stiffness. For example, if rotation is used to control the probing direction and the probe is inserted in an S-shaped trajectory, the opposing curvatures along the probe might straighten out, causing tearing of the tissue. Additionally, rotation at the needle base can cause a lag between its base and the tip, which might hinder the control of needle insertion (chapter 2). Rotations of the entire needle can be avoided if they are confined to the distal end of the probe. Such twisting might even occur in insect probes as discussed above. However, a biomimetic needle that exploits both the push-pull insertion mechanism and a soft region in its distal end for steering, is yet to be developed.

\section{The use of sensors during probing}

An important part of probing that has so far not been addressed, is how insects detect their targets. Finding the targets is, of course, essential and requires a number of different sensors. First, the insects need to determine if a substrate contains a target, which is done by the use of long-range olfactory, visual, and thermal cues. ${ }^{82-86}$ These are detected by sensors that are generally concentrated on the head of the animal, such as in the eyes, antennae, and mouthparts. ${ }^{84,86-88}$ After settling on a substrate, the insect needs to determine the location of the host and a suitable probing site. This is done by contact chemoreception (taste) and mechanoreception (touch). Both types of receptors are generally found on the structures enveloping the probe, namely the labium in the mosquitoes and hemipterans and the sheaths in wasps. ${ }^{84,87,89}$ Before initiation of probing, insects usually brush the substrate with the tips of the structures protecting the probe, presumably to decide on a suitable probing spot. ${ }^{1,23,90}$

Additionally, parasitic wasps often use very sensitive mechanoreceptors on their legs, the subgenual organs, to detect vibrations induced by their hosts in the substrate. ${ }^{20,91-93}$ This can be either done by active echolocation or passive 'listening' to the hosts. Active echolocation involves the wasps touching the substrate with their antennae in what has been described as 'drumming'. ${ }^{94}$ These motions are thought to transmit the vibrations of the body of the insects into the substrate. ${ }^{95}$ The vibrations get reflected by the host and detected by the wasps with their subgenual organs. ${ }^{94,95}$ Alternatively, the wasps use the subgenual organs to detect the host vibrations induced when, for example, chewing the substrate. ${ }^{20,91}$ In the kinematic experiments (chapter 3), we observed that the wasps were more likely to probe in substrates that were hiding a live larva than a dead or an immobile one, which agrees with earlier observations of probing in D. longicaudata. ${ }^{96}$ It has been shown that, at least in lepidopteran leaf-mining hosts, the vibrational cues depend on the 
stage and activity of the host. ${ }^{97,98}$ The parasitoids can potentially use this information when deciding whether to probe or not. However, the vibrational cues are influenced by the substrate they pass through. Complex (heterogeneous) substrates might introduce too much noise which would occlude detailed host information and further studies elucidating the effect of substrates on vibrational host sensing. ${ }^{92}$ The wasps might use the vibrations only to ascertain that living hosts are hiding within the substrate. ${ }^{96}$ For example, in chapter 3 the larvae were always out of the wasps' reach, but that did not stop them from probing. This suggests that the olfactory, visual, and vibrational cues are used to determine the rough location of the target, while its exact location and quality are assessed during the insertion of the probe inside the substrate.

To secure the food or to lay the egg, probes need to be guided to the correct location. The chemical and thermal cues produced by the targets presumably disperse and form gradients within the substrate, which can be detected by the probing insects. Probes of mosquitoes, hemipterans, and wasps possess specialized sensors that respond to chemical (gustatory or olfactory) or mechanical cues. ${ }^{97,99-107}$ The chemo- and mechanoreceptors are anatomically distinct, although a dual function for receptors found on the ovipositor has been suggested. ${ }^{100,108,109}$ Parasitic wasps ovipositors seem well equipped with sensors used to detect various cues, including inorganic chemicals such as $\mathrm{CO}_{2},{ }^{110}$ various organic compounds emitted by the hosts or conspecifics, $86,108,111-114$ and potentially also the stresses and strains within the ovipositor walls, ${ }^{109}$ and stiffness of the substrate. ${ }^{50}$

Receptors can be found in both outer (mosquitoes, wasps) and inner walls (hemipterans, wasps) of the probes. ${ }^{84,88,105,106,115-118}$ While the inner-wall receptors are used to monitor the transport through the probe, those in the outer walls of the probe presumably help directing the probe to the target. In the ovipositors of parasitic wasps, the density and distribution of the outer-wall receptors depends on the sensor type and varies between the valves and along their length.

The chemoreceptors are generally concentrated on the distal part of the dorsal ovipositor valve, although some sensors are also present in the tips of the ventral valves. e.g. $88,100,118$ This arrangement indicates, that the dorsal valve is the main sensing element, which might be because this valve presumably moves less during probing. The valve appears stationary with respect to the body (chapter 4), and might be moved at lower frequencies and amplitudes in the substrate compared to the ventral valves. The smaller movements might reduce both the potential risk of damage to the sensory neurons and noise in the sensory system. The concentration of the chemoreceptors at the tip indicates that the wasps detect the gradients of sensory cues during probing, but not when the probe is stationary in the substrate. 
The probes are presumably steered towards the highest concentration of the cue by bending within the substrate. In case the target is not reached, the insects (wasps, mosquitoes, and hemipterans) retract their probe and reinserted it into the substrate following a different pathway ${ }^{55,119}$ (chapter 3). This indicates that probing insects might remember the temporally distributed sensory input during probing, which would facilitate finding of the target.

The mechanoreceptors are located along the entire length of the ovipositor and might be used to monitor the state (stress and strains due to bending) of the probe. .e. ${ }^{39,101}$ If wasps can remember also the mechanosensory output, they might use this information to create a spatial map of the relevant sensory cues inside the substrate. Such a map might further facilitate the finding of the target.

Little is known about how insects actually locate their targets. To my knowledge, temporal insertion patterns of probing insects have never been investigated, while our kinematic study is the only quantitative study on the spatial distribution ovipositor insertions (chapter 3). Individual wasps showed no directional preferences, presumably because we did not offer them a localized target, but hid several moving hosts underneath the substrate. When the target was not reached, the wasps generally reinserted their ovipositors in a new direction (i.e. did not follow the same trajectory of insertion). This indicates that the insects might indeed remember the cue intensity and location in the substrate during probing. This is not unlikely, as parasitic wasps are capable of associative learning that facilitates the finding of hosts. ${ }^{120,121}$ To better understand the role of sensors during probing, future studies should explore the physiological sensitivity of the receptors and the possible role of memory during probing. This might also bring insights in the functioning of the invertebrate brain and the evolution of memory.

SENSORS IN MAN-MADE PROBES. The target location is in surgical procedures known in advance, so sensors are not needed for this purpose, but rather for diagnosing the tissue. Similar to insects, the man-made probes can be equipped with sensors for detecting the chemical (e.g. $\mathrm{pH}$, oxygen, lipids) and physical parameters (e.g. temperature, blood flow, force). Such sensors might be made from optical fibres, which are already used in medical diagnostics tools. ${ }^{122}$ Additionally, the optical fibres can be made very thin and would therefore not hinder further minimization of the needle prototypes. Currently, up to four sensory channels could be used in the IC London needle prototype, ${ }^{60,62,123}$ while the TU Delft prototype does not possess any physical channels that could fit a sensors. ${ }^{57}$ However, as the TU Delft prototype consists of wires, perhaps some (or most) of them could be substituted with equally thin optical fibres without the loss of functionality. The sensors have 
to my knowledge only been implemented in the IC London prototype in a proofof-concept study ${ }^{62}$ and further work needs to be done in this direction. I expect that the functionality of the man-made probes will be greatly expanded with novel sensors that allow for characterization of the tissue and thus facilitate the diagnostics.

\section{Retraction of the ovipositor}

We can distinguish two types of retractions-a partial retraction where the animal keeps the distal part of the probe inside the substrate, and a full retraction where the probe is completely removed from the substrate before reinsertion. A partial retraction has been directly observed in wasps (chapter 3) and mosquitoes, ${ }^{\text {e.g. }} 55$ but not in hemipterans. Nevertheless, the substrate insertion trajectories of hemipterans suggest that these insects also partially retract their probes to explore a different region of the substrate. ${ }^{1,119}$ The discussed probe tip serrations that increase friction between the probe and the substrate, do not appear to hinder probe retraction in mosquitoes, parasitic wasps, or hemipterans. These animals are strong enough to retract (and extract) their probes at will. However, the friction between the substrate and the probe might slow down the speed of retraction. As mentioned, during probing the insects are stationary for long periods of time and might be an easy prey. For example, parasitic wasps have been observed to be preyed upon by ants, ${ }^{21}$ while mosquitoes and assassin bugs run the risk of being swatted by their hosts. It might therefore be important for the insect to be able to quickly disengage from the substrate if danger is perceived. In our kinematic study, we observed that the wasps always retract the ovipositor with the ventral valves fully retracted (Fig. S5). This presumably reduced the friction between the substrate and the serrated tips of the ventral valves and eased the retraction process. I hypothesize that this might be an adaptation to speed up the retraction of the probe and that similar friction-reducing mechanisms might also be present in other probing insects.

\section{Perspectives}

In this thesis, I elucidated the versatility of slender, multi-element probes, by studying the parasitic wasp D. longicaudata. Its probing capabilities and the kinematics and actuation of the support that insects employ the push-pull mechanism, which reduces the risk of buckling and damage to the probe. Furthermore, the study of the structural and material properties of the valves revealed a novel implementation of a previously hypothesized steering mechanism. The mechanism that evolved in D. longicaudata might be particularly suited for both probing in stiff substrates and steering within them. The steering capabilities of D. longicaudata were, how- 
ever, only partially explained in this thesis. It is still not completely clear how the wasps insert their ovipositors in any direction with respect to their body orientation or follow complex trajectories within the substrate. We hypothesize, this may be achieved by rotation or twisting of the ovipositor and further biomechanical studies are needed to test this hypothesis.

An aspect of the probing process that was only partially addressed in this thesis, is the role of the substrate in the probing process. The probe interaction with the substrate can either facilitate or hinder the insect probing capabilities, particularly steering. Additional studies are needed to bring insights into how the probe shape and kinematics depends on substrate properties such as stiffness.

Understanding the push-pull mechanism, its dependence on the substrate properties, and the steering mechanisms of insect probes might be important for the development of slender and steerable man-made probes. Such probes could be in the future used in minimally invasive surgical procedures. Current needle prototypes are multi-element and implement the reciprocal movements to reduce the risk of buckling, but they are either not sufficiently slender or cannot achieve sufficient curvatures to be used in practice. Studies of probing in insects may help in combining the high slenderness and curvature demands on man-made needles.

Another aspect that has not been addressed in this thesis is the sensory function of the insect probes. To successfully reach the targets, insects may benefit from the ability to remember the sensory output during probing. Investigating the sensory perception during probing might therefore reveal insect memory capabilities and could lead to advances in the arthropod neurobiology.

Sensors are present on most insect probes and are presumably important to reach the target hidden within the substrates. In man-made probes, sensors are not needed to reach the target, but probes with sensory capabilities would be useful in diagnostics. Currently, sensor implementation in steerable needles is still in its infancy, but I foresee a rapid development of multifunctional sensory needles in the coming decade. 


\section{References}

[1] Pollard DG (1973). Plant penetration by feeding aphids (Hemiptera, Aphidoidea): a review. Bulletin of Entomological Research 62, 631.

[2] KRenN HW \& Aspöck H (2012). Form, function and evolution of the mouthparts of blood-feeding Arthropoda. Arthropod Structure \& Development 41, 101-118.

[3] Pennacchio F \& Strand MR (2006). Evolution of developmental strategies in parasitic Hymenoptera. Annual Review of Entomology 51, 233-258.

[4] Brożé J, Mróz E, Wylȩżé D, Depa $€$, \& WȩGierek P (2015). The structure of extremely long mouthparts in the aphid genus Stomaphis Walker (Hemiptera: Sternorrhyncha: Aphididae). Zoomorphology 134, 431-445.

[5] Le LANNIC J \& NÉNON JP (1999). Functional morphology of the ovipositor in Megarhyssa atrata (Hymenoptera, Ichneumonidae) and its penetration into wood. Zoomorphology 119, 73-79.

[6] Heatwole H, Davis DM, \& Wenner AM (1962). The behaviour of Megarhyssa, a genus of parasitic hymenopterans (Ichneumonidae: Ephialtinae). Zeitschrift fur Tierpsychologie - Journal of Comparative Ethology 19, 652-664.

[7] Montoya P, Benrey B, Barrera JF, Zenil M, Ruiz L, \& Liedo P (2003). Oviposition Behavior and Conspecific Host Discrimination in Diachasmimorpha longicaudata (Hymenoptera: Braconidae), a Fruit Fly Parasitoid. Biocontrol Science and Technology 13, 683-690.

[8] Auclair JL (1963). Aphid feeding and nutrition. Annual Review of Entomology 8, 439-490.

[9] Quicke DLJ, Fitton MG, Tunstead JR, Ingram SN, \& Gaitens PV (1994). Ovipositor structure and relationships within the Hymenoptera, with special reference to the Ichneumonoidea. Journal of Natural History 28, 635-682.

[10] Robinson GG (1939). The mouthparts and their function in the female mosquito, Anopheles maculipennis. Parasitology 31, 212.

[11] Scali M, Pusch TP, Breedveld P, \& Dodou D (2017). Needle-like instruments for steering through solid organs: A review of the scientific and patent literature. Proceedings of the Institution of Mechanical Engineers, Part H: Journal of Engineering in Medicine 231, 250-265.

[12] Dogangil G, Davies BL, \& Rodriguez Y Baena F (2010). A review of medical robotics for minimally invasive soft tissue surgery. Proceedings of the Institution of Mechanical Engineers, Part H: Journal of Engineering in Medicine 224, 653-679.

[13] Krenn HW, Plant JD, \& Szucsich NU (2005). Mouthparts of flower-visiting insects. Arthropod Structure and Development 34, 1-40.

[14] Raven JA (1983). Phytophages of xylem and phloem: a comparison of animal and plant sapfeeders. Advances in Ecological Research 13, 135-234.

[15] Sмiтн EL (1963). Biosystematics and morphology of Symphyta - II Biology of gall-making nematine sawflies in the California region. Annals of the Entomological Society of America 63, 36-51.

[16] LeE SJ, KIM BH, \& LeE JY (2009). Experimental study on the fluid mechanics of blood sucking in the proboscis of a female mosquito. Journal of biomechanics 42, 857-864.

[17] KrenN HW \& KRIstensen NP (2004). Evolution of proboscis musculature in Lepidoptera. European Journal of Entomology 101, 565-575.

[18] Krenn HW (2000). Proboscis musculature in the butterfly Vanessa cardui (Nymphalidae, Lepidoptera): settling the proboscis recoiling controversy. Acta Zoologica 81, 259-266.

[19] Bauder JAS, Handschuh S, Metscher BD, \& Krenn HW (2013). Functional morphology of the feeding apparatus and evolution of proboscis length in metalmark butterflies (Lepidoptera: Riodinidae). Biological Journal of the Linnean Society 110, 291-304.

[20] Vilhelmsen L, Isidoro N, Romani R, Basibuyuk HH, \& Quicke DLJ (2001). Host location and oviposition in a basal group of parasitic wasps: the subgenual organ, ovipositor apparatus and associated structures in the Orussidae (Hymenoptera, Insecta). Zoomorphology 121, 63-84. 
[21] Kundanati L \& Gundiah N (2014). Biomechanics of substrate boring by fig wasps. Journal of Experimental Biology 217, 1946-1954.

[22] SAKes A, Dodou D, \& Breedveld P (2015). Buckling prevention strategies in nature as inspiration for improving percutaneous instruments: a review. Bioinspiration \& Biomimetics 11.

[23] Gordon RM \& Lumsden WHR (1939). A study of the behaviour of the mouth-parts of mosquitoes when taking up blood from living tissue together with some observations on the ingestion of microfiliarie. Annals of tropical medicine and parasitology 33, 259-278.

[24] Foster WA \& Walker ED (2002). Mosquitoes (Culicidae). In G Mullen \& D LanCe, eds., Medical and veterinary entomology, 597, Elsevier Science.

[25] Vilhelmsen L (2003). Flexible ovipositor sheaths in parasitoid Hymenoptera (Insecta). Arthropod Structure \& Development 32, 277-287.

[26] Jones JC \& PILITT DR (1973). Blood-feeding behavior of adult Aedes aegypti mosquitoes. Biological Bulletin 145, 127-139.

[27] Izumi H, Yajima T, Aoyagi S, Tagawa N, Arai Y, Hirata M, \& Yorifuji S (2008). Combined harpoonlike jagged microneedles imitating mosquito's proboscis and its insertion experiment with vibration. IEEJ transactions on electrical and electronic engineering 3, 425-431.

[28] Okamura AM, Simone C, \& O'Leary MD (2004). Force modeling for needle insertion into soft tissue. IEEE Transactions on Biomedical Engineering 51, 1707-1716.

[29] Wang Y, Chen RK, Tai BL, McLaughlin PW, \& Shin AJ (2014). Optimal needle design for minimal insertion force and bevel length. Medical Engineering and Physics 36, 1093-1100.

[30] Casanova F, Carney PR, \& Sarntinoranont M (2014). In vivo evaluation of needle force and friction stress during insertion at varying insertion speed into the brain. Journal of Neuroscience Methods 237, 79-89.

[31] SCHUdDER GCE (1971). Comparative morphology of insect genitalia. Annual Review of Entomology 16, 379-406.

[32] Pollard DG (1969). Directional control of the stylets in phytophagous Hemiptera. Proceedings of the Royal Entomological Society of London. Series A, General Entomology 44, 173-185.

[33] Sмітн EL (1970). Evolutionary morphology of the external insect genitalia. 2. Hymenoptera. Annals of the Entomological Society of America 63, 1-27.

[34] VINCENT JFV \& KING MJ (1995). The mechanism of drilling by wood wasp ovipositors. Biomimetics 3, 187-201.

[35] Spradbery JP (1970). Host finding by Rhyssa persuasoria (L.) in ichneumonid parasite of siricid woodwasps. Animal Behaviour 18, 103-114.

[36] Leyva JL, Browning HW, \& Gilstrap FE (1991). Effect of host fruit species, size, and color on parasitization of Anastrepha ludens (Diptera: Tephritidae) by Diachasmimorpha longicaudata (Hymenoptera: Braconidae). Environmental Entomology 20, 1469-1474.

[37] Griffiths RB \& GoRdon RM (1952). An apparatus which enables the process of feeding by mosquitoes to be observed in the tissues of a live rodent; together with an account of the ejection of saliva and its significance in malaria. Annals of tropical medicine and parasitology 46, 311-319.

[38] Aoyagi S, Izumi H, \& Fukuda M (2007). Biodegradable polymer needle with various tip angles and effect of vibration and surface tension on easy insertion. 2007 IEEE 20th International Conference on Micro Electro Mechanical Systems (MEMS) 397-400.

[39] Ghara M, Kundanati L, \& Borges RM (2011). Nature's Swiss Army knives: ovipositor structure mirrors ecology in a multitrophic fig wasp community. PloS one 6, e23642.

[40] Elias LG, Kjellberg F, Antoniolli Farache HF, Almeida EAB, Rasplus Jy, Cruaud A, Peng YQ, Yang DR, \& Pereira Santinelo AR (2018). Ovipositor morphology correlates with life history evolution in agaonid fig wasps. Acta Oecologica 90, 109-116.

[41] Hudson A (1970). Notes on the piercing mouthparts of three species of mosquitoes (Diptera: Culicidae) viewed with te scanning electron microscope. The Canadian Entomologist 102, 501-9. 
[42] Pollard DG (1970). The mechanism of stylet movement in Psylla mali Schmidberger (Homoptera: Psyllidae). Zoological Journal of the Linnean Society 49, 295-307.

[43] Polidori C, García AJ, \& Nieves-Aldrey JL (2013). Breaking up the wall: metal-enrichment in ovipositors, but not in mandibles, co-varies with substrate hardness in gall-wasps and their associates. PloS one 8, e70529.

[44] Quicke DLJ, Wyeth P, Fawke JD, Basibuyuk HH, \& Vincent JFV (1998). Manganese and zinc in the ovipositors and mandibles of hymenopterous insects. Zoological Journal of the Linnean Society 124, 387-396.

[45] Belshaw R, Grafen A, \& Quicke DLJ (2003). Inferring life history from ovipositor morphology in parasitoid wasps using phylogenetic regression and discriminant analysis. Zoological Journal of the Linnean Society 139, 213-228.

[46] GAULD ID (1988). Evolutionary patterns of host utilization by ichneumonoid parasitoids (Hymenoptera: Ichneurnonidae and Braconidae). Biological Journal of Linnean Society 35, 351-377.

[47] Greany P (1986). In vitro culture of hymenopterous larval endoparasitoids. Journal of Insect Physiology 32, 409-419.

[48] Quicke DLJ (2015). The braconid and ichenumonid parasitoid wasps: biology, systematics, evolution and ecology. John Wiley \& Sons, Ltd, first edn.

[49] Baltazar BCR (1964). The genera of prasitic Hymenoptera in the Philippines, part 2. Pacific Insects 6, 15-67.

[50] Gal R, Kaiser M, Haspel G, \& Libersat F (2014). Sensory arsenal on the stinger of the parasitoid jewel wasp and its possible role in identifying cockroach brains. PLoS one 9, e89683.

[51] Hocking H (1968). Studies on the biology of Rhyssa persuasoria (L.) (Hymenoptera: Ichenumonidae) incorporating and X-ray technique. Journal of the Australian Entomological Society 7, 1-5.

[52] RoBerTson PL (1968). A morphological and functional study of the venom apparatus in representatives of some major groups of Hymenoptera. Australian Journal of Zoology 16, 133.

[53] Hermann HR (1971). Sting autotomy, a defensive mechanism in certain social Hymenoptera. Insectes Sociaux 18, 111-120.

[54] Zhao ZL, Zhao HP, Ma GJ, Wu CW, Yang K, \& Feng XQ (2015). Structures, properties, and functions of the stings of honey bees and paper wasps: a comparative study. Biology open 4, 921928.

[55] Choumet V, Attout T, Chartier L, Khun H, Sautereau J, Robbe-Vincent A, Brey P, Huerre M, \& BAIN O (2012). Visualizing non infectious and infectious Anopheles gambiae blood feedings in naive and saliva-immunized mice. PloS one 7 , e50464.

[56] Powell JA \& Turner WJ (1975). Observations on oviposition behavior and host selection in Orussus occidentalis (Hymenoptera: Siricoidea). Journal of the Kansas Entomological Society 48, 299-307.

[57] Scali M, Pusch T, Breedveld P, \& Dodou D (2017). Ovipositor-inspired steerable needle: design and preliminary experimental evaluation. Bioinspiration \& Biomimetics 13, 016006.

[58] Frasson L, Ko Sy, Turner A, Parittotokkaporn T, Vincent JF, \& Rodriguez y Baena F (2010). STING: a soft-tissue intervention and neurosurgical guide to access deep brain lesions through curved trajectories. Proceedings of the Institution of Mechanical Engineers, Part H: Journal of Engineering in Medicine 224, 775-788.

[59] Frasson L, Ferroni F, Ko SY, Dogangil G, \& Rodriguez y Baena F (2011). Experimental evaluation of a novel steerable probe with a programmable bevel tip inspired by nature. Journal of Robotic Surgery 6, 189-197.

[60] Ko SY \& y BAena F (2013). Toward a miniaturized needle steering system with path planning for obstacle avoidance. IEEE Transactions on Biomedical Engineering 60, 910-917.

[61] Burrows C, Liu F, Leibinger A, Secoli R, \& Rodriguez y Baena F (2017). Multi-target planar needle steering with a bioinspired needle design. In G Boschetti \& A GASPARETto, eds., Advances in Italian Mechanism Science, 51-60, Cham: Springer International Publishing. 
[62] Virdyawan V, Oldfield M, \& Rodriguez y Baena F (2018). Laser Doppler sensing for blood vessel detection with a biologically inspired steerable needle. Bioinspiration \& biomimetics 13, 026009.

[63] Parittotokkaporn T, Frasson L, Schneider A, Huq S, Davies BL, Degenaar P, Biesenack J, \& Rodriguez y BAena FM (2009). Soft tissue traversal with zero net force: Feasibility study of a biologically inspired design based on reciprocal motion. Proceedings of the 2008 IEEE International Conference on Robotics and Biomimetics 80-85.

[64] Yamaguchi H, Tanaka H, Hasegawa M, Tokuda M, Asami T, \& Suzuki Y (2012). Phytohormones and willow gall induction by a gall-inducing sawfly. New Phytologist 196, 586-595.

[65] Hajek AE, Nielsen C, Kepler RM, Long SJ, \& Castrillo L (2013). Fidelity among Sirex woodwasps and their fungal symbionts. Microbial ecology 65, 753-762.

[66] GiLmour JW (1964). The life cycle of the fungal symbiont of Sirex noctilio. New Zealand Forest Service 80-89.

[67] Coutts MP \& Dolezal JE (1969). Emplacement of fungal spores by the woodwasp, Sirex noctilio, during oviposition. Forest Science 15, 412-416.

[68] Stillwell MA (1966). Woodwasps (Siricidae) in conifers and the associated fungus, Stereum chailletii, in eastern Canada. Forest Science 12, 121-128.

[69] Greany PD, Hawke SD, Carlysle TC, \& Anthony DW (1977). Sense organs in the ovipositor of Biosteres (Opius) longicaudatus a parasite of the Caribbean fruit fly Anastrepha suspensa. Annals of the Entomological Society of America 70, 319-321.

[70] Elias LG, Teixeira SP, KJellberg F, \& Santinelo Pereira RA (2012). Diversification in the use of resources by Idarnes species: bypassing functional constraints in the fig-fig wasp interaction. Biological Journal of the Linnean Society 106, 114-122.

[71] Compton S \& NefDt R (1988). Extra-long ovipositors in chalcid wasps: some examples and observations. Antenna 12, 102-105.

[72] Roesthuis RJ, Van Veen YR, Jahya A, \& Misra S (2011). Mechanics of needle-tissue interaction. 2011 IEEE/RSJ International Conference on Intelligent Robots and Systems 2557-2563.

[73] Dai W, PAn L, Lu Y, Jin L, \& Zhang C (2014). External morphology of the mouthparts of the whitebacked planthopper Sogatella furcifera (Hemiptera: Delphacidae), with special reference to the sensilla. Micron 56, 8-16.

[74] Coвben RH (1978). Evolutionary trends in heteroptera: Part II. Mouthpart-structures and feeding strategies. Wageningen, The Netherlands: H. Veenman \& Zonen B. V.

[75] Quicke DLJ (1991). Ovipositor mechanics of the braconine wasp genus Zaglyptogastra and the ichneumonid genus Pristomerus. Journal of Natural History 25, 971-977.

[76] Quicke DLJ, Fitton M, \& Harris J (1995). Ovipositor steering mechanisms in braconid wasps. Journal of Hymenoptera Research 4, 110-120.

[77] Hermann HR \& Blum MS (1967). The morphology and histology of the hymenopterous poison apparatus. II. Pogonomyrmex badius (Formicidae). Ann. Entomol. Soc. Am. 60, 661-668.

[78] Copland MJW \& KIng PE (1972). The structure of the female reproductive system in the Torymidae (Hymenoptera: Chalcidoidea). Transactions of the Royal Entomological Society of London 124, 191-212.

[79] Scali M, Kreeft D, Breedveld P, \& Dodou D (2017). Design and evaluation of a wasp-inspired steerable needle. Proc. SPIE 10162, Bioinspiration, Biomimetics, and Bioreplication 2017 10162, 1016207.

[80] Swaney PJ, Burgner J, Gilbert HB, \& Webster RJ (2013). A flexure-based steerable needle: high curvature with reduced tissue damage. IEEE Transactions on Biomedical Engineering 60, 906-909.

[81] Minhas DS, Engh JA, Fenske MM, \& Riviere CN (2007). Modeling of needle steering via dutycycled spinning. Annual International Conference of the IEEE Engineering in Medicine and Biology Proceedings 2756-2759.

[82] TAKKen W \& Verhulst NO (2013). Host preferences of blood-feeding mosquitoes. Annual Review of Entomology 58, 433-453. 
[83] Segura DF, Nussenbaum AL, \& Viscarret MM (2016). Innate host habitat preference in the parasitoid Diachasmimorpha longicaudata: functional significance and modifications through learning. PloS One 11, 0152222.

[84] Brozek J \& Bourgoin T (2013). Morphology and distribution of the external labial sensilla in Fulgoromorpha (Insecta: Hemiptera). Zoomorphology 132, 33-65.

[85] Maekawa E, Aonuma H, Nelson B, Yoshimura A, Tokunaga F, Fukumoto S, \& Kanuka H (2011). The role of proboscis of the malaria vector mosquito Anopheles stephensi in host-seeking behavior. Parasites and Vectors 4, 10.

[86] Vinson SB (1976). Host selection by insect parasitoids. Annual Review of Entomology 21, 109-133.

[87] Lee RMKW \& Craig DA (2009). Fine structure of the sense organs on the labella and labium of the mosquito Aedes aegypti (L .). The Open Entomology Journal 3, 7-17.

[88] Consoli FL, Kitajima EW, \& Postali Parra JR (1999). Sensilla on the antenna and ovipositor of the parasitic wasps Trichogramma galloi Zucchi and T. pretiosum Riley (Hym., Trichogrammatidae). Microscopy Research and Technique 45, 313-324.

[89] Hatfield LD \& Frazier JL (1980). Ultrastructure of the labial tip sensilla of the tarnished plant bug, Lygus lineolaris (P. de Beauvois) (Hemiptera: Miridae). International Journal of Insect Morphology and Embryology 9, 59-66.

[90] NéNON JP, Kacem N, \& LANNic LJ (1997). Structure, sensory equipment, and secretions of the ovipositor in a giant species of Hymenoptera: Megrahyssa atrata F. (Ichneumonidae, Pimplinae). The Canadian Entomologist 129, 789-799.

[91] BROAD GR \& Quicke DL (2000). The adaptive significance of host location by vibrational sounding in parasitoid wasps. Proceedings of the Royal Society B 267, 2403-2409.

[92] MeYhÖFER R \& CASAS J (1999). Vibratory stimuli in host location by parasitic wasps. Journal of Insect Physiology 45, 967-971.

[93] Vilhelmsen L, Turrisi GF, \& Beutel RG (2008). Distal leg morphology, subgenual organs and host detection in Stephanidae (Insecta, Hymenoptera). Journal of Natural History 42, 1649-1663.

[94] Henaut A \& Guerdoux J (1982). Location of a lure by the drumming insect Pimpla instigator (Hymenoptera, Ichneumonidae). Experientia 38, 346-347.

[95] Henaut A (1990). Study of the sound produced by Pimpla instigator (Hymenoptera, Ichneumonidae) during host selection. Entomophaga 35, 127-139.

[96] LaWrenCE PO (1981). Host vibration - A cue to host location by the parasite Biosteres longicaudatus. Oecologia 48, 249-251.

[97] MeYhöFER R, CASAS J, \& Dorn S (1994). Host location by a parasitoid using leafminer vibrations: characterizing the vibrational signals produced by the leafmining host. Physiological Entomology 19, 349-359.

[98] MeYhofer R, CASAS J, \& Dorn S (1997). Vibration-mediated interactions in a host-parasitoid system. Proceedings of the Royal Society B: Biological Sciences 264, 261-266.

[99] Dweck HKM, Gadallah NS, \& Darwish E (2008). Structure and sensory equipment of the ovipositor of Habrobracon hebetor (Say) (Hymenoptera: Braconidae). Micron 39, 1255-1261.

[100] Sнан ZA (2012). Morphology, ultrastructure, and probable functions of the sense organs on the ovipositor stylets of the hymenoptran parasitoid, Venturia canescens (Gravenhorst). Microscopy research and technique 75, 876-883.

[101] Zhang L, Feng YQ, Ren LL, Luo YQ, Wang F, \& Zong Sx (2014). Sensilla on antenna, ovipositor and leg of Eriborus applicitus (Hymenoptera: Ichneumonidae), a parasitoid wasp of Holcocerus insularis staudinger (Lepidoptera: Cossidae). Acta Zoologica 96, 253-263.

[102] BRown PE \& ANDERson M (1998). Morphology and ultrastructure of sense organs on the ovipositor of Trybliographa rapae, a parasitoid of the cabbage root fly. Journal of Insect Physiology 44, 1017-1023. 
[103] Crnjar R, Angioy A, Pietra P, J S, Liscia A, \& Barbarossa Tomassini I (1989). Electrophysiological studies of gustatory and olfactory responses of the sensilla on the ovipositor of the apple maggot fly, Rhagoletis pomonella Walsh. Italian Journal of Zoology 56, 41-46.

[104] Сноо YM, Buss GK, TAN K, \& Leal WS (2015). Multitasking roles of mosquito labrum in oviposition and blood feeding. Frontiers in Physiology 6, 1-11.

[105] Won Jung J, Baeck SJ, Perumalsamy H, Hansson BS, Ahn YJ, \& Kwon HW (2015). A novel olfactory pathway is essential for fast and efficient blood-feeding in mosquitoes. Scientific Reports $5,1-10$.

[106] BACKUS EA (1988). Sensory systems and behaviours which mediate hemipteran plant-feeding: a taxonomic overview. Jouornal of Insect Physiology 34, 151-165.

[107] Oten KLF, Cohen AC, \& Hain FP (2014). Stylet bundle morphology and trophically related enzymes of the hemlock woolly adelgid (Hemiptera: Adelgidae). Annals of the Entomological Society of America 107, 680-690.

[108] Van Lenteren JC, Ruschioni S, Romani R, van Loon JJA, Qiu YT, SMid HM, Isidoro N, \& Bin $\mathrm{F}$ (2007). Structure and electrophysiological responses of gustatory organs on the ovipositor of the parasitoid Leptopilina heterotoma. Arthropod Structure \& Development 36, 271-276.

[109] Hawke SD, Farley RD, \& Greany PD (1973). The fine structure of sense organs in the ovipositor of the parasitic wasp, Orgilus lepidus Muesebeck. Tissue and Cell 5, 171-184.

[110] YAdAV P \& Borges RM (2017). The insect ovipositor as a volatile sensor within a closed microcosm. Journal of Experimental Biology 220, 1554-1557.

[111] VAN Lenteren JC (1975). The development of host discrimination and the prevention of superparasitism in the parasite Pseudeucoila bochei Weld (Hym.: Cynipidae). Netherlands Journal of Zoology 26, 1-83.

[112] Goubault M, Cortesero AM, Paty C, Fourrier J, Dourlot S, \& Le Ralec A (2011). Abdominal sensory equipment involved in external host discrimination in a solitary parasitoid wasp. Microscopy research and technique 74, 1145-1153.

[113] Ruschioni S, Van Loon JJA, Smid HM, \& Van Lenteren JC (2015). Insects can count: sensory basis of host discrimination in parasitoid wasps revealed. PLoS One 10, e0138045.

[114] Rogers D (1972). The ichneumon wasp Ventura canescens: oviposition and avoidance of superparasitism. Entomologia Experimentalis et Applicata 15, 190-194.

[115] Zhao L, Dai W, Zhang C, \& Zhang Y (2010). Morphological characterization of the mouthparts of the vector leafhopper Psammotettix striatus (L.) (Hemiptera: Cicadellidae). Micron 41, 754-759.

[116] Ahmad A, Kaushik S, Ramamurthy VV, Lakhanpaul S, Ramani R, Sharma KK, \& VidYARTHI AS (2012). Mouthparts and stylet penetration of the lac insect Kerria lacca (Kerr) (Hemiptera:Tachardiidae). Arthropod Structure \& Development 41, 435-441.

[117] Ahmed T, Zhang Tt, He Kl, Bai Sx, \& Wang Zy (2013). Sense organs on the ovipositor of Macrocentrus cingulum Brischke (Hymenoptera: Braconidae): their probable role in stinging, oviposition and host selection process. Journal of Asia-Pacific Entomology 16, 343-348.

[118] Ganesalingam VK (1972). Anatomy and histology of the sense organs of the ovipositor of the Ichneumonid wasp, Devorgilla canescens. Journal of Insect Physiology 18, 1857-1867.

[119] Leopold RA, Freeman TP, Buckner JS, \& Nelson DR (2003). Mouthpart morphology and stylet penetration of host plants by the glassy-winged sharpshooter, Homalodisca coagulata, (Homoptera: Cicadellidae). Arthropod Structure \& Development 32, 189-199.

[120] Rehman A (2010). Host selection behaviour of aphid parasitoids (Aphidiidae: Hymenoptera). Journal of Plant Breeding and Crop 2, 299-311.

[121] Turlings TC, Tumlinson JH, Lewis WJ, \& Vet LE (1989). Beneficial arthropod behavior mediated by airborne semiochemicals. VIII. Learning of host-related odors induced by a brief contact experience with host by-products in Cotesia marginiventris (Cresson), a generalist larval parasitoid. Journal of Insect Behavior 2, 217-225. 
[122] Mignani AG \& Baldini F (1999). Biomedical sensors using optical fibres. Reports on Progress in Physics 59, 1-28.

[123] Frasson L, Neubert J, Reina S, Oldfield M, Davies BL, \& Rodriguez Y Baena F (2010). Development and validation of a numerical model for cross-section optimization of a multi-part probe for soft tissue intervention. 32nd Annual International Conference of the IEEE EMBS 32, 3202-3205. 



\section{Summaries}

\section{Acknowledgements}

About the author

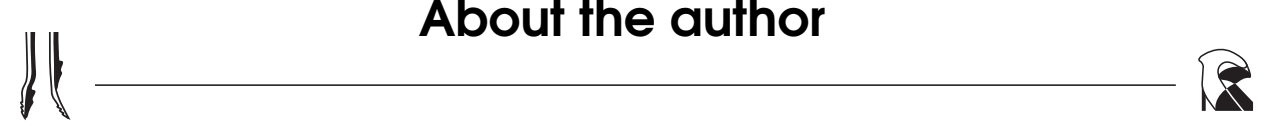




\section{Summary}

Insects such as mosquitoes, true bugs, and parasitic wasps, probe for resources hidden in various substrates. The resources are often, located deep within the substrate and can only be reached with long and thin (slender) probes. Such probes can, however, easily bend or break (buckle) when pushed inside the substrate, which makes probing a challenging task. Nevertheless, the mentioned insects use their probes repeatedly throughout their lifetime without apparent damage. Furthermore, the probes are also used for sensing the targets, can be steered during insertion, and can transport both fluids (e.g. blood, phloem sap) and eggs. Insect probes seem highly versatile structures that satisfy many functional requirements, including buckling avoidance, steering, sensing, and transport. Similar requirements also hold for minimally invasive medical procedures, where slender tools are used to minimize damage to the patient. Understanding the probing process in insects can bring insights in the insect ecology and evolution and it may also help in the development of novel surgical tools. In this thesis, I focus on the mechanical and motor adaptations of insect probing, while other aspects are only briefly discussed.

In chapter 2, we review the literature on the probing structures and their operating principles across mosquitoes, parasitic wasps, and hemipterans. Probes are either modified mouthparts (mosquitoes, true bugs) or special tubular outgrowths of the abdomen (parasitic wasps). Despite having different developmental origins, the probes share three major morphological characteristics, which may reflect the shared functional requirements of buckling avoidance and steering: (i) the probes consist of multiple, interconnected elements that can slide along each other, (ii) the probe diameters are very small, which leaves no space for internal musculature, and (iii) the distal ends (tips) of the probe elements are asymmetric and often bear various serrations, hooks, bulges, or notches.

How such slender multi-element probes avoid buckling during insertion has been hypothesized in the so-called push-pull mechanism. According to this mechanism, the probe is inserted into the substrate by reciprocal movements of the elements. The insects therefore simultaneously push on some of the probe elements, while pulling on the others. The tip serrations are directed such, that they primarily increase the friction upon pulling of the elements. This puts the pulled elements under tension and makes them effectively stiffer in bending (like when pulling a rope). The elements under tension can serve as guides along which the other elements are pushed inside the substrate without the risk of buckling. The insect alternates the pushing and pulling between the elements to incrementally insert the probe in the substrate. This mechanism has, however, never been quantified in insects and it was hitherto unknown whether the animals rely on it during probing. 
The probe tip asymmetry presumably facilitates steering. The asymmetric tip geometry leads to asymmetric reaction forces from the substrate on the tip during insertion, which push the probe tip sideways into a curved path. Controlling the tip geometry therefore allows for control of probing direction. Although offsetting the elements by sliding already changes the shape of the probe tip, these changes might be too small to induce the necessary change of probing direction. A number of mechanisms that enhance the tip asymmetry during the sliding of the elements have been suggested. However, few mechanisms have been observed or studied in vivo, so it is not completely clear how insects steer with their probes. Additionally, the effect of the substrate on both the steering and insertion mechanisms is unknown.

To understand the biomechanics of insect probing, we investigated the probing behaviour of the braconid parasitic wasp Diachasmimorpha longicaudata. This is an ideal species for studying the buckling avoidance and steering, because it: (i) possess a slender ovipositor several millimetres in length, (ii) probes into solid material (e.g. citrus fruits), and (iii) attack fruit-fly larvae that are freely moving within the substrate (i.e. steering can be expected). The ovipositor of $D$. longicaudata is similar to other hymenopterans and consists of three interconnected elements (valves), one dorsal and two ventral ones. The interconnection is a tongue-and-groove mechanism, which allows for sliding of the valves, but prevents their separation. The ovipositor has an asymmetric tip-the distal end of the dorsal valve is enlarged (bulge), while the ventral valve tips have harpoon-like serrations. Additionally, just proximal to the bulge of the dorsal valve, the ovipositor is characteristically bent in an S-shape. This seems to be a feature present only in D. longicaudata and closely related species. The wasps also possess a pair of sheaths that envelop the ovipositor at rest and throughout most of the probing process, but do not penetrate into the substrate.

In chapter 3, we studied the kinematics of ovipositor insertion into translucent, artificial substrates of various stiffnesses. Ovipositor insertion was filmed in a threecamera setup, which allowed us to reconstruct the ovipositor insertion in $3 \mathrm{D}$, while also monitoring the orientation of the insect's body. We discovered that the wasps can explore a wide range of the substrate by probing in any direction with respect to their body orientation from a single puncture point. Probing range and speed decreased with increasing substrate stiffness. Wasps used two strategies of ovipositor insertion. In soft substrates, all ovipositor valves were pushed inside the substrate at the same time. In stiff substrates, wasps always moved the valves alternatively, presumably employing the hypothesized push-pull mechanism. We observed that ovipositors can follow curved trajectories inside the substrate. Detailed kinematic 
analysis revealed that the ovipositors followed a curved path during probing with protracted ventral valve(s). In contrast, probing with protracted dorsal valve resulted in straight trajectories. We linked the changes in the probing direction to the shape changes in the ovipositor tip. When the ventral valves were protracted, they curved towards the dorsal valve, resulting in an enhanced bevel which presumably caused a change in insertion direction.

In chapter 4, we investigated the above described steering mechanism by quantifying the bending stiffness (three point bend test) and the geometry (high-resolution computer tomography) of the ovipositor in D. longicaudata. Additionally, we qualitatively assessed the material composition of the valves using fluorescence imaging. The thick dorsal valve bulge might be stiff and could straighten the S-shaped region of the ovipositor during the valve offset, causing bending of the tip. We discovered that the S-shaped region of the ovipositor is significantly softer than its neighbouring regions, which is mostly due to the presence of resilin in the S-shaped region of the ventral valve. Resilin is a rubber-like protein and reduces the stiffness of the otherwise heavily sclerotized valves. Additionally, we showed that the ventral valves have a higher bending stiffness than the dorsal valve along most of their length. The exception is presumably the bulge on the dorsal valve-although we could not directly measure its bending stiffness, its geometrical properties show that it is the thickest (and therefore stiffest) region in the distal end of the ovipositor.

Outside the substrate, offsetting of the valves in any direction (i.e. pro- or retraction of the ventral valves) caused a straightening of the S-shaped region of the ovipositor and a curving towards the dorsal side. However, during probing in a substrate, such curving was only observed upon protraction of the ventral valves. We hypothesize this is due to the interaction of the ovipositor with the substrate. Namely, the bevelled ventral valve tips generate substrate reaction forces that promote dorsal curving, while the bevelled tip of the dorsal valve generates substrate forces that promote ventral bending. The interaction between the ventral and dorsal valves straightens the S-shaped region of the ovipositor and enhances dorsal curving. This therefore facilitates strong shape changes of the tip only upon protraction of the ventral valves, while counteracting the ventral curving of the dorsal valve. These opposing mechanisms presumably result in an approximately straight protraction of the dorsal valve.

In chapters 2ãnd 3 we describe how the wasps use the reciprocal valve movements when probing in stiff substrates. As such substrates presumably require strong forces during insertion, the reciprocal valve movements may indeed serve to avoid buckling. However, how the valves are actuated or the forces generated during probing have never been quantified. In chapter 5, we therefore investigated the 
ovipositor base and the muscles driving the movements of the valves. At the base, the valves attach to plate-like structures that are interconnected with a series of linkages. The muscles attach to these plates and can move them with respect to each other. Such movements also result in the movements of the valves. To analyse the mechanics of this linked system, we performed high-resolution computer tomography scans of wasps in different stages of the probing cycle. This allowed us to compare the configurational changes of the basal plates to the valve offset, and measure the muscle cross-sections and attachment sites. We also calculated the muscle moment arms and estimated the forces and moments of the most relevant musculature actuating the ovipositor movements, by assuming a tensile muscle stress previously reported for insect muscles. For the ventral valves only, we also calculated the forces the valves can exert onto the substrate. The dorsal valve can only be moved by moving the base that is linked inside the abdomen, and therefore force estimation could not be made.

The displacement magnitude of the basal plates corresponded to the valve offset, indicating that the valves are indeed moved due to the changes in the arrangement of the basal plates. We also showed that the ventral valve plates move most during the probing cycle, while the magnitude of the dorsal valve plate movements is much smaller. This suggests that the ventral valves move along the dorsal valve, while the dorsal valve moves together with the abdomen during probing. Additionally, in the situation where the animal keeps its abdomen stationary, we estimated the maximal forces actuating the ventral valves. The estimated maximal pushing forces can be higher than the estimated buckling load of the unsupported ovipositor outside the substrate. Assuming the maximal pushing forces are required during probing, antibuckling mechanisms are needed to avoid damaging the ovipositor. Buckling can be limited (prevented) by either supporting the ovipositor outside the substrate with additional sheaths, employing the push-pull mechanism, or both. Subtracting the maximal estimated pushing and pulling forces on the ventral valves, results in a net pushing force that is very close to the buckling threshold of the ovipositor, albeit still slightly higher. The sheaths, although being flexible, might provide the additional support if needed.

In this thesis, I show that multi-element probes are inserted into the substrate using reciprocal movements of the individual elements. These movements appear to be necessary in stiff substrates, which presumably require high pushing forces on a single element during probing. This is in accordance with the hypothesis that reciprocal valve movements serve as an anti-buckling mechanism. Additionally, such valve movements are also important for steering of the probe during insertion. The valve offset controls the shape of the probe tip and therefore the net substrate re- 
action forces that result in bending of the probe. Wasps evolved special structures that enhance the shape changes of their ovipositor tips and facilitate steering. Our findings may be interesting for a broad range of audiences. Entomologists, evolutionary biologists, and ecologists may find them useful when studying the diversification of probing insects, their evolutionary success, or their ecological interactions (e.g. insect-plant, parasite-host). The anti-buckling and steering mechanisms may be helpful when developing novel, man-made probes. These mechanisms allow for minimization of the probe thickness and accurate steering control, which minimizes substrate damage during probing. Our findings may be particularly useful in the development of slender, steerable needles for minimally invasive surgery. 


\section{Povzetek}

Komarji, polkrilici (npr. listne uši) in parazitske ose so žuželke, ki iščejo hrano zase ali za svoje potomce $\mathrm{v}$ raznovrstnih substratih s pomočjo podolgovatih struktur - sond. Ker je hrana pogosto skrita globoko v substratu, morajo biti sonde zelo dolge in tanke. Zato se $\mathrm{z}$ lahkoto nepovratno zvijejo (v nadaljevanju uklonijo, angl. buckle) ali celo zlomijo. Vse to pomeni, da je iskanje hrane za te žuželke precej zahtevno opravilo. Kljub temu pa sonde uporabljajo vse življenje, večinoma brez opaznih poškodb. Žuželke lahko s sondami med raziskovanjem substratov krmilijo (to je: določajo smer vrtanja) in sonde uporabljajo tudi za zaznavanju tarč znotraj substratov. Uporabljajo jih tudi za transport tekočin (npr. kri, floemski sok) ali jajčec. To pomeni, da so sonde vsestransko uporabne in izpolnjujejo veliko funkcionalnih potreb, kot so, na primer: preprečevanje uklona (angl. buckling avoidance), krmiljenje (angl. steering), služijo pa tudi kot čutilo in za transport snovi. Podobne funkcionalne zahteve srečamo tudi pri minimalno invazivnih kirurških posegih, kjer se za omejevanje poškodb pri bolnikih med operacijami uporabljajo tanka orodja. Razumevanje uporabe tankih sond za raziskovanje različnih substratov pri žuželkah je zanimivo tako iz biološkega vidika, saj lahko prinese nova spoznanja o ekologiji in evoluciji žuželk, kot tudi za inženirstvo, saj lahko pripomore k razvoju novih kirurških orodij. V doktorski disertaciji sem se osredotočil predvsem na mehanske in motorične prilagoditve žuželčjega raziskovanja substratov, medtem ko se ostalih vidikov razprava le bežno dotakne.

V 2. poglavju smo pregledali obstoječo literaturo o strukturah, ki jih za sondiranje uporabljajo komarji, polkrilci in parazitske ose. Kot sonde se uporabljajo: ali prilagojeni obustni aparat (komarji, polkrilci) ali pa posebni cevasti izrastki na zadku žuželke (parazitske ose). Čeprav imajo sonde žuželk različne evolucijske in razvojne izvore, so si podobne $\mathrm{v}$ treh glavnih morfoloških značilnostih, ki mogoče odražajo skupne funkcionalne zahteve o omejevanju uklona in krmiljenju: (i) sonde so sestavljene iz več medsebojno povezanih preobraženih okončin, v nadaljevanju elementov, ki lahko drsijo drug ob drugem, (ii) zaradi zelo majhnih premerov v sondah ni prostora za mišičje, in (iii) distalni deli posameznih elementov (konice) so asimetrični in pogosto nazobčani, odebeljeni, ali pa opremljeni z utori.

Hipoteza, kako se tanke strukture, sestavljene iz več elementov med vstavljanjem $\mathrm{v}$ substrat izognejo uklonu/zlomu, je bila postavljena $\mathrm{v}$ tako imenovanem potisnovlečnem (angl. push-pull) mehanizmu. Glavna značilnost tega mehanizma je, da je sonda vstavljena v substrat $\mathrm{z}$ recipročnim premikanjem posameznih elementov. Žuželke tako nekatere od elementov potiskajo, istočasno pa vlečejo preostale. Zobci na konicah elementov so obrnjeni tako, da najbolj pripomorejo k trenju ob njihovem potegu. Zato so elementi ob potegu izpostavljeni nateznim silam, ki jih, podobno 
kot $\mathrm{v}$ napeti vrvi, naredijo bolj toge (angl. stiffer) in odporne proti upogibanju. Elementi pod natezno napetostjo služijo tudi kot vodila za preostale elemente, ki jih žuželka potisne globlje v substrat. Slednji se zato izognejo uklonu ali zlomu. Žuželke naj bi izmenično potiskale in vlekle posamezne elemente in tako sonde vstavljale v substrate postopoma. Do zdaj ni bilo znano, ali se žuželke poslužujejo zgoraj opisanega mehanizma, saj pri teh živalih še nikoli ni bil kvantificiran.

Asimetrična oblike konice sonde žuželk predvidoma olajša njeno krmiljenje, saj med njenim vstavljanjem vzpostavi asimetrične reakcijske sile znotraj substrata. Te asimetrične sile vodijo konico po ukrivljeni poti proč od ravne smeri. Nadzorovanje oblike konice sonde tako omogoča nadzorovanje smeri vrtanja. Vzdolžen zamik elementov sonde (angl. offset) spremeni njeno konico. Možno je, da sam zamik elementov ne zadostuje za spremembo smeri vrtanja. V literaturi predlagajo kar nekaj mehanizmov, ki povečajo asimetrijo sondne konice med zamikom njenih elementov. Le nekaj od teh mehanizmov pa so opazili in preučili in vivo in zato ni čisto jasno, kako žuželke s svojimi sondami pravzaprav krmilijo. Poleg tega pa tudi ni znano, kako na mehanizme vstavljanja in krmiljenja vpliva sam substrat.

Da bi razumeli biomehaniko vstavljanja žuželčjih sond v substrat, smo preučili vrtanje pri parazitski osi Diachasmimorpha longicaudata (Braconidae). Samice os te vrste so odlične za preučevanje tega, kako se izogniti uklonu sonde in z njo krmiliti saj: (i) imajo sondo (leglico) dolgo več milimetrov pri kateri je razmerje med dolžino in debelino zelo veliko, (ii) vrtajo $\mathrm{v}$ trdno sadje, na primer, $\mathrm{v}$ citruse, in (iii) napadajo ličinke sadnih muh, ki se svobodno gibljejo znotraj substratov. Tako lahko pričakujemo, da ose med vrtanjem z leglico tudi krmilijo. Leglica pri $D$. longicaudata je podobna leglicam ostalih os in je sestavljena iz treh medsebojno povezanih elementov, enega hrbtnega in dveh trebušnih. Povezava med elementi ima v prerezu obliko lastovičjega repa, ki omogoča drsenje elementov drug ob drugem in hkrati preprečuje njihovo razdvojitev. Konica leglice je asimetrična, saj je distalni del hrbtnega elementa odebeljen (angl. bulge), medtem ko sta konici trebušnih elementov nazobčani s harpuni podobnimi zobci. Poleg tega pa je celotna leglica tik pred odebelitvijo hrbtnega elementa zavita v značilno obliko črke S. Tak zavoj je prisoten le pri D. longicaudata in njej sorodnih vrstah. Leglico objema par struktur, ki jima skupno pravimo nožnica (angl. sheath). Ta leglico objema, ko je ose ne uporabljajo in tudi med samim vrtanjem. Ob vrtanju se nožnica zvije in ostane na površini substrata.

V 3. poglavju, smo preučili kinematiko vstavljanja leglice v umetne, prosojne substrate različnih trdnosti (angl. stiffness). Vstavljanje leglice smo posneli s tremi kamerami, kar nam je omogočilo rekonstrukcijo vrtanja $v$ treh dimenzijah in spremljanje položaja osinega telesa med vrtanjem v substrat. Odkrili smo, da lahko 
ose z leglico vrtajo v katerokoli smer proč od svojega telesa. Iz ene same točke na površini lahko tako raziščejo velik del substrata. Velikost območja vrtanja in hitrost vstavljanja leglice sta se s povečanjem trdnosti substrata zmanjšali. Trdnost substrata pa je vplivala tudi na strategijo vrtanja z leglico. V mehkih substratih so ose leglico vstavile tako, da so hkrati potiskale vse elemente leglice globlje v substrat. V trdnih substratih pa so ose posamezne elemente leglice vedno premikale posamično in sklepamo, da so uporabile zgoraj opisani potisno-vlečni mehanizem. Opazili smo tudi, da lahko ose z leglico vrtajo po ukrivljenih poteh znotraj substrata. Podrobna analiza vrtanja je razkrila, da je leglica sledila ukrivljeni poti, ko sta bila med vrtanjem v ospredju njena trebušna elementa. Vrtanje s hrbtnim elementom $\mathrm{v}$ ospredju je vodilo $\mathrm{k}$ ravnim potem vstavljanja leglice. $\mathrm{S}$ to raziskavo smo povezali spremembo smeri vrtanja z leglico s spremembo oblike njene konice. Ob potisku trebušnih elementov pred hrbtnega sta se trebušna elementa ukrivila proti hrbtnemu, kar je povečalo asimetrijo konice in predvidoma spremenilo smer vrtanja.

V 4. poglavju smo preučili zgoraj opisani mehanizem krmiljenja pri vrsti D. longicaudata. Izmerili togost leglice s tritočkovnim ukrivitvenim testom (angl. three point bending test) in kvantificirali obliko leglice s pomočjo visoko-ločljive računalniške tomografije (angl. high-resolution computer tomography). Poleg tega smo tudi kvalitativno ocenili materialno sestavo leglice s fluorescenčno mikroskopijo. Odebeljen del hrbtnega elementa leglice je verjetno tog in lahko izravna ukrivljeno S-obliko leglice ob zamiku elementov, kar verjetno povzroči ukrivitev konice leglice. Odkrili smo, da je del leglice oblikovan v črko $S$ veliko mehkejši od preostalih delov leglice, najverjetneje zaradi prisotnosti posebnega proteina, rezilina (angl. resilin), $\mathrm{v}$ trebušnih elementih. Rezilin je protein podoben gumi, ki zmanjša togost visoko sklerotiziranih (in zato togih) elementov leglice. Poleg tega smo pokazali, da so trebušni deli leglice bolj togi od večjega dela hrbtnega elementa. Izjema je verjetno le odebeljena konica hrbtnega elementa, ki nam je sicer ni uspelo izmeriti s tritočkovnim ukrivitvenim testom. Iz njene oblike pa lahko sklepamo, da je ta del leglice najbolj tog, saj je tudi najdebelejši.

Izven substrata, zamik trebušnih elementov leglice izravna del leglice oblikovan v obliki črke $S$ in povzroči ukrivitev celotne leglice v hrbtni smeri. Tako ukrivitev leglice med vrtanjem znotraj substrata pa smo opazili le ob potisku trebušnih elementov pred hrbtnega. Sklepamo, da je to zaradi vpliva substrata na leglico. Poševna konica trebušnih elementov verjetno ustvari reakcijske sile substrata v smeri, ki ojača hrbtno ukrivitev leglice. Zaradi medsebojnega vpliva hrbtnega in trebušnih elementov, se del leglice, ki je oblikovan v črko $S$, ob zamiku elementov izravna in tudi povzroči hrbtno ukrivitev. Oba mehanizma skupaj olajšata spremembo oblike 
konice leglice, toda le med potiskanjem trebušnih elementov. Zaradi poševne oblike konice hrbtnega elementa, ta ob potisku v substratu povzroči reakcijske sile, ki vodijo v trebušno zvijanje leglice. Proti tej smeri pa deluje hrbtno ukrivljanje leglice zaradi samega zamika elementov. Potisk hrbtnega elementa tako vodi v približno raven potisk hrbtnega elementa.

V 2. in 3. poglavju smo opisali, kako ose recipročno premikajo posamezne elemente leglice med vrtanjem $\mathrm{v}$ trdne substrate. Ker so za vrtanje $\mathrm{v}$ takšne substrate predvidoma potrebne velike sile, je mogoče, da recipročno premikanje posameznih elementov leglice res omeji uklon leglice in tako prepreči morebitne poškodbe. Kako in s kakšnimi silami ose sploh premikajo posamične elemente, še nikoli ni bilo kvantificirano. V 5. poglavju smo zato preučili proksimalen del leglice (bazo) in mišičje, s katerimi ose upravljajo posamezne elemente. Slednji so v bazi pritrjeni na več ploščatih in medsebojno povezanih struktur (bazalnih plošč), na katere je pripeto tudi mišičje. Zaradi medsebojnih povezav premik posamezne plošče verjetno premakne tudi z njo povezan element. Mehaniko tega povezanega sistema smo analizirali z računalniško tomografijo visoke ločljivosti. Ose smo posneli v različnih položajih vstavljanja leglice v substrat. Tako smo lahko primerjali spremembo lege bazalnih plošč z zamikom posameznih elementov leglice. Poleg tega pa smo izmerili, kje se mišičje pripenja na zunanji skelet žuželke in kvantificirali presek mišičja. To nam je omogočilo, da smo izračunali velikost navornih ročic in ocenili sile in navore najbolj pomembnih mišic, ki premikajo leglico. V izračunih smo domnevali, da je specifična napetost žuželčjih mišic enaka tisti, najdeni v literaturi. Samo za trebušne elemente smo izračunali tudi silo, s katero delujejo na substrat. Sile na hrbtni element ni bilo mogoče oceniti, saj ga ose lahko premikajo samo s premikanjem celotnega zadka.

Velikost premikanja bazalnih plošč se je skladala z velikostjo zamika posameznih elementov, kar nakazuje, da se elementi res premikajo zaradi premikanja bazalnih plošč. Pokazali smo tudi, da imajo največjo amplitudo premikanja plošče, povezane $\mathrm{s}$ trebušnima elementoma, medtem ko je velikost premika plošč, povezanih s hrbtnim elementom veliko manjša. To nakazuje, da trebušna elementa prosto drsita ob hrbtnem, ki se premika skupaj z zadkom žuželke. Ocenili smo tudi najvišje sile, $\mathrm{s}$ katerimi žuželka premika trebušna elementa v primeru, ko njen zadek miruje. Sile, s katerimi so trebušni elementi lahko potisnjeni v substrat, so višje od ocenjenega praga uklona (angl. buckling threshold) leglice, ki je zunaj substrata. Če domnevamo, da so ravno te najvišje sile nujne med vrtanjem, potem ose potrebujejo posebne mehanizme, ki omejijo ali preprečijo uklon/zlom leglice. To lahko dosežejo z oporo dela leglice, ki je zunaj substrata (nožnica), z uporabo potisno-vlečnega mehanizma ali z obema mehanizmoma hkrati. Če odštejemo najvišje potisne in vlečne 
sile, ki delujejo na trebušne elemente, je velikost rezultante zelo blizu ocenjenemu pragu uklona, čeravno še zmeraj malo višja. Mogoče je potrebna dodatna opora dosežena z nožnico, čeprav se slednja ob vrtanju zvije in ostane na površju.

V disertaciji sem pokazal, da žuželke sonde $\mathrm{z}$ več elementi vstavljajo v substrate s pomočjo recipročnega premikanja posameznih elementov. Tako (recipročno) gibanje je očitno nujno $\mathrm{v}$ trdnih substratih, kjer so potrebne visoke potisne sile na posamezne elemente. Te ugotovitve so $\mathrm{v}$ skladu s hipotezo, da recipročno gibanje elementov služi za preprečevanje neželenega upogibanja sonde. Poleg tega sem pokazal, da je recipročno gibanje elementov pomembno tudi za krmiljenje sonde žuželk. Zamik elementov nadzira obliko konice sonde in s tem tudi reakcijske sile substrata, ki določajo smer vrtanja. Med evolucijo so ose razvile posebne strukture, ki okrepijo spremembe oblike konice njihovih leglic in olajšajo krmiljenje med vrtanjem. Odkritja, predstavljena v disertaciji, so zanimiva za širok krog ljudi. Uporabna so lahko za entomologe, evolucijske biologe in ekologe, ki raziskujejo biodiverziteto vrtajočih žuželk, njihov evolucijski uspeh, ali pa njihove interakcije $\mathrm{z}$ rastlinami ali gostitelji. Poleg tega so opisani mehanizmi krmiljenja in izogibanja uklonu lahko koristni pri razvoju umetnih sond. Omogočajo miniaturizacijo in natančno krmiljenje sonde, kar zmanjšuje poškodbe substrata med vrtanjem. Vsa ta odkritja bi lahko bila koristna pri razvoju tankih igel za minimalno invazivne kirurške posege. 


\section{Samenvatting}

Verschillende insecten, zoals muggen, wantsen en parasitaire wespen, boren naar voedselbronnen in diverse substraten. De voedselbronnen zitten vaak diep verborgen in het substraat en kunnen alleen bereikt worden met een lange en dunne (slanke) boor. Slanke boren kunnen echter makkelijk buigen of knikken, en daardoor beschadigen, wanneer ze in het substraat worden geduwd. Dit maakt het boren met een slanke boor dan ook een lastige opgave. De eerder genoemde insecten zijn echter in staat om zonder schade hun slanke boor herhaaldelijk te gebruiken. Daarnaast bevatten de boren ook sensoren om het doel te vinden en kunnen de boren naar het doel gestuurd worden. Bovendien kunnen de boren gebruikt worden om vloeistoffen (bloed of floëemvloeistof) uit te zuigen of om eieren af te zetten. Deze insectboren zijn dus zeer veelzijdig en voldoen aan heel veel functie-eisen zoals stevigheid, om knikken te voorkomen, stuurbaarheid, waarnemingsvermogen en transportmogelijkheden. Vergelijkbare eisen worden ook gesteld aan instrumenten die gebruikt worden bij minimaal-invasieve medische ingrepen, waarbij slanke instrumenten worden gebruikt om schade aan de patiënt te minimaliseren. Als we begrijpen hoe de boren van deze insecten werken, begrijpen we niet alleen de ecologie en de evolutionaire geschiedenis van deze dieren beter, maar kunnen we deze kennis ook gebruiken voor de ontwikkeling van nieuwe minimaal-invasieve medische instrumenten. In dit proefschrift ligt de nadruk voornamelijk op de mechanische eigenschappen van de legboor van een parasitaire wesp en hoe deze wordt aangedreven. Andere elementen die belangrijk zijn voor het functioneren van de legboor worden zijdelings behandeld.

In hoofdstuk 2 geven we een overzicht over wat er op dit moment in de literatuur bekend is over de borende structuren van muggen, parasitaire wespen en wantsen, en hoe zij werken. Muggen en wantsen boren met speciaal aangepaste monddelen terwijl parasitaire wespen een specifieke buisvormige structuur (legboor) aan het achterlijf (abdomen) gebruiken. Ondanks dat de monddelen en de legboor uit totaal verschillende delen van het lichaam ontwikkelen zijn ze op drie kenmerken vergelijkbaar. Dit kan er op duiden dat deze kenmerken bepaald worden door de functie-eisen die aan deze borende structuren worden gesteld zoals het voorkomen van knikken en de stuurbaarheid van de boor. Deze kenmerken zijn: (i) de boren bestaan uit meerdere, onderling verbonden delen die over de lengteas langs elkaar kunnen schuiven, (ii) de diameter van de boren is zeer klein, waardoor er geen ruimte is voor interne spieren, en (iii) de punt van de boren zijn asymmetrisch en hebben over het algemeen kartelingen, haakjes, of knobbels.

Eén manier waarop knikken in een slanke borende structuur met meerdere elementen voorkomen kan worden is beschreven in het zogenaamde 'push-pull me- 
chanism' (druk-en-trek mechanisme). Volgens de theorie achter dit mechanisme beweegt de boor in het substraat als gevolg van het heen-en-weer bewegen van de individuele elementen, waarbij op het zelfde moment sommige elementen naar voren worden geduwd, terwijl aan andere elementen wordt getrokken. De uitsteeksels op de punt van de boor zijn dusdanig gericht dat zij ervoor zorgen dat de elementen waaraan getrokken wordt vast blijven zitten. Hierdoor komen deze elementen onder spanning te staan waardoor dit deel van de boor stijver wordt, net als bij een touw waar aan getrokken wordt. Deze stijve elementen kunnen nu als een geleide worden gebruikt voor de elementen die dieper het substraat in worden geduwd, waardoor het risico op knikken sterk vermindert. Door vervolgens het deel wat het diepst in het substraat gestoken is onder spanning te zetten en te gebruiken als geleide voor een ander legboordeel kan de boor stapsgewijs veilig verder het substraat in worden geduwd. Tot nu toe is dit mechanisme puur theoretisch en nog niet getoetst. Het is daarom onzeker of insecten inderdaad gebruik maken van dit mechanisme.

De asymmetrische vorm van de punt van de boor draagt hoogstwaarschijnlijk bij aan het sturingsmechanisme. Deze vorm leidt tot asymmetrische reactiekrachten van het substraat op de punt waardoor de punt zijdeling wordt weggeduwd wat resulteert in een gebogen pad. Als de asymmetrie van de punt gecontroleerd verandert kan worden, kan dus ook de richting van boren worden gecontroleerd. Hoewel de vorm van de punt al enigszins verandert door alleen de voor-achterwaartse verschuiving van de legboordelen, is het te verwachten dat deze veranderingen te klein zijn om de boor in een scherpe bocht te duwen. Er zijn een aantal hypotheses over hoe de boor wel in scherpere bochten kan worden gestuurd, maar deze zijn zelden in levende dieren gezien of geanalyseerd. Het is dus nog in hoge mate onduidelijk hoe de insecten hun boren precies sturen. Daarnaast is het ook onbekend of het materiaal waarin geboord wordt invloed heeft op hoe goed de dieren kunnen boren en sturen in het substraat.

Om de werking van het boren door insecten beter te begrijpen hebben wij het boren van de braconide, parasitaire wesp Diachasmimorpha longicaudata bestudeerd. Dit is een ideale soort om het boren te bestuderen omdat deze wespen (i) een slanke legboor hebben van enkele millimeters lengte, (ii) boren in massief substraat (citrusvruchten) en (iii) hun eieren leggen in fruitvlieglarven die vrij door de vruchten kruipen, waardoor verwacht kan worden dat ze de legboor moeten kunnen sturen. De legboor van $D$. longicaudata lijkt op die van andere dieren uit de orde van de vliesvleugeligen (Hymenoptera) en bestaat uit drie onderling verbonden delen, een aan de rugzijde (dorsaal) en twee aan de buikzijde (ventraal). De legboordelen zijn verbonden via een soort messing-en-groef verbinding, die het glijden van de ele- 
menten langs elkaar mogelijk maakt, maar losraken voorkomt. De legboor heeft een asymmetrische punt met een knobbel op de dorsale zijde en zaagtanden aan de ventrale zijde. Net achter de puntregio zit een karakteristieke S-vormige bocht in de legboor, die alleen bij $D$. longicaudata en nauw verwante soorten wordt gezien. Buiten het lichaam is de legboor omgeven door een tweeledige schede die de legboor beschermd en ondersteund, maar die niet in het substraat wordt geduwd.

In hoofdstuk 3 beschrijven we de kinematica (bewegingen) van de legboor tijdens het boren in doorzichtige, kunstmatige substraten van verschillende stijfheid (stevigheid). De boorbewegingen (inserties) werden gefilmd met drie camera's, wat het mogelijk maakte om de bewegingen tijdens de insertie drie dimensionaal te reconstrueren. Tegelijkertijd werd met een derde camera ook de oriëntatie van het lichaam van de wesp gefilmd. Hiermee hebben we aangetoond dat de wespen vanuit één insertiepunt een zeer groot deel van het substraat kunnen bereiken en ongeacht hun lichaamspositie in alle richtingen kunnen boren. Het bereik en de snelheid van boren nam af met toegenomen stijfheid van het substraat. We namen ook waar dat de wespen twee manieren van boren gebruikte. In zachte substraten werden alle legboordelen tegelijkertijd in het substraat geduwd. In stijve substraten bewogen de wespen de legboordelen alternerend, wat wijst op het gebruik van het 'push-pull' mechanisme. Bovendien werd duidelijk dat de wespen de legboor in gebogen paden konden sturen in het substraat. Gedetailleerde analyse van de bewegingspatronen toonde aan dat de legboor een gebogen pad ging volgen als de ventrale legboordelen gemiddeld vaker het meest vooruitgestoken waren dan het dorsale legboordeel. In het geval dat het dorsale legboordeel gemiddeld het meest vooruitgestoken was leidde dit tot een rechte, niet gebogen, boorpad. We hebben deze verschillen in boorrichting gekoppeld aan een vormverandering van de legboorpunt. Als de ventrale legboordelen voor het dorsale legboordeel uitsteken, buigt hun punt in de richting van de dorsale zijde, wat resulteert in een grotere asymmetrie die waarschijnlijk resulteert in de door ons waargenomen richtingsverandering.

In hoofdstuk 4 hebben we het hierboven beschreven sturingsmechanisme van D. longicaudata bestudeerd door de stijfheid (buigzaamheid) te meten van de legboor en de individuele legboordelen. Dit is gedaan met behulp van een zogenaamd driepuntsbuigexperiment. Daarnaast hebben we de geometrie van de legboor bepaald met behulp van hoge resolutie computer tomografie (CT). Tot slot hebben we kwalitatief de materiaalsamenstelling van de legboor bepaald met behulp van fluorescentiemicroscopie. De dikke knobbel op de dorsale zijde van de legboor zorgt ervoor dat de legboor op die plek moeilijk buigt. Als de knobbel langs de S-bocht komt te liggen door verplaatsing van de legboordelen zorgt dat ervoor dat de to- 
tale legboor zich strekt en de S-bocht verdwijnt. Omdat het middelste deel van de S-bocht gestrekt wordt kan dit leiden tot een de buiging van de punt van de ventrale legboordelen in dorsale richting. Het gedeelte van de legboor met de S-bocht is significant flexibeler dan de punt en de rest van de legboor, wat voornamelijk veroorzaakt wordt door de aanwezigheid van resiline, een rubberachtig eiwit wat het normaal gesproken zeer stijve exoskelet soepeler maakt. Daarnaast was het ook duidelijk dat de ventrale legboordelen over vrijwel de gehele lengte stijver zijn dan het dorsale legboordeel. De uitzondering hierop is waarschijnlijk de dikke knobbel op het dorsale legboordeel, hoewel we dit niet direct konden meten. De geometrie toont echter aan dat dit het dikste en daarom stijfste deel is van het uiteinde van de legboor.

In lucht resulteert het verschuiven van de ventrale delen naar voren of naar achteren altijd in het strekken (en dus verdwijnen) van de S-bocht en het naar dorsaal buigen van de punt van de legboor. Tijdens boren in een substraat zien we de buiging omhoog echter alleen bij het naar voren schuiven van de ventrale delen. We veronderstellen dat dit het gevolg is van de interactie tussen de legboor en het substraat. Het schuin aflopende uiteinde van de ventrale delen zorgt voor reactiekrachten van het substraat die de punt naar dorsaal duwen, terwijl het schuin aflopende uiteinde van het dorsale deel juist zorgt voor krachten die de punt naar ventraal duwen. De interactie tussen de ventrale en dorsale delen van de legboor zorgt dat de S-bocht verdwijnt en dat de legboorpunt naar dorsaal wordt geduwd. Als de ventrale delen vooruit worden geschoven zorgt de asymmetrische punt voor een versterking van de dorsale buiging, terwijl als het dorsale deel het meeste uitsteekt de reactiekrachten de dorsale buiging juist tegenwerken, wat resulteert in een recht pad van de legboor.

In hoofdstuk 2 en 3 beschrijven we hoe wespen gebruik maken van alternerende bewegingen van de legboordelen tijdens het boren in stijve substraten. Aangezien het kan worden aangenomen dat er bij gewoon duwen grote krachten nodig zijn om in deze stijve substraten te boren, is het aannemelijk dat de alternerende bewegingen bijdragen aan het voorkomen van knikken van de legboor. Hoe groot de krachten zijn die de wesp kan uitoefenen is echter nog nooit bepaald. In hoofdstuk 5 hebben we daarom gekeken naar de basis van de legboor die zorgt voor de beweging van de legboordelen en de spieren die daar aanhechten. Aan de basis van de legboor zitten de schuifbare delen van de legboor via een serie van gewrichten verbonden aan plaatvormige structuren. De spieren hechten aan op de platen. Door de platen te bewegen, bewegen ze indirect de schuivende delen van de legboor zelf. Om goed inzicht te krijgen in de werking van dit mechanisme hebben we hoge resolute computer tomografie (CT) afbeeldingen gemaakt van wespen met de schuifbare delen 
van de legboor in verschillende posities, zodanig dat we een overzicht kregen van de mogelijke bewegingen binnen de basis van de legboor. Hierdoor waren we in staat om de veranderingen in het systeem waar te nemen en de dwarsdoorsnede door de spieren en hun aanhechtingen te bepalen. Daarnaast bepaalden we de momentarmen, krachten en momenten van de spieren die een rol spelen in de aansturing van de legboor en zijn onderdelen. De kracht werd bepaald door schaling van reeds bekende treksterktes van andere insectspieren. Alleen voor de ventrale delen van de legboor konden de krachten die de wesp kan uitoefenen op het substraat bepaald worden. Onze analyse toonde aan dat het dorsale legboordeel alleen verplaatst kan worden samen met de basis van de legboor die verbonden zit aan het achterlijf (abdomen). Aangezien het abdomen op verschillende manieren verplaatst kan worden konden we geen eenduidige schatting maken van de kracht die door het dorsale deel kan worden uitgeoefend.

De afstand waarover de platen van de basis verplaatst kunnen worden correleert sterk met de afstand waarover de schuivende delen van de legboor kunnen verplaatsen, wat aangeeft dat deze inderdaad gekoppeld zijn. We laten zien dat vooral de ventrale delen van de legboor bewegen tijdens het boren en dat het dorsale element nauwelijks verplaatst ten opzichte van het lichaam. Dit suggereert dat de ventrale elementen langs het dorsale element schuiven, terwijl het dorsale element alleen met het achterlijf meebeweegt. Voor de situatie waarbij de wesp het achterlijf volkomen stil houdt hebben we de maximale kracht berekend die door de ventrale delen kan worden uitgeoefend. De maximale krachten die op de legboor kunnen worden uitgeoefend overschrijden de kracht die kan leiden tot het knikken van de legboor als deze niet ondersteund wordt. Als we ervan uitgaan dat deze maximale krachten worden uitgeoefend tijdens het boren, zijn ondersteunende structuren of mechanismen nodig om schade aan de legboor te voorkomen. In het geval van de legboor kan deze buiten het substraat ondersteund worden door de schedes, maar ook door het gebruiken van het 'push-pull' mechanisme, of beide. De netto drukkracht die we berekenen als we de geschatte maximale trek- en duwkrachten van de ventrale delen van elkaar aftrekken is iets hoger dan de kracht die leidt tot knikken en dus is de combinatie van het 'push-pull' mechanisme en gebruik van de schede waarschijnlijk voldoende om schade aan het externe deel van de legboor te voorkomen.

In dit proefschrift laat ik zien hoe boren met meerdere schuivende legboordelen in het substraat kunnen worden gestoken door deze alternerend naar voren te schuiven. Deze manier van boren lijkt essentieel voor het boren in stijve substraten, waar waarschijnlijk anders hoge duwkrachten op de elementen voor vereist zijn. Dit is in overeenstemming met de hypothese dat de alternerende beweging het knikken van 
de legboor kan voorkomen. Daarnaast is de alternerende beweging ook van belang voor het sturen van de legboor tijdens het boren. Het vooruitsteken van een of enkele elementen zorgt voor een vormverandering in de legboorpunt wat in combinatie met reactiekrachten van het substraat leidt tot het buigen van de legboor. Tijdens de evolutie van de wespen zijn verschillende structuren ontstaan die de vormverandering en het effect hiervan op de baan van de legboor vergroten.

Onze resultaten zijn relevant voor een breed publiek. Entomologen, evolutionair biologen en ecologen kunnen onze resultaten gebruiken als zij kijken naar de adaptieve radiatie van borende insecten, hun evolutionaire succes en de ecologische interacties (bv insect-plant, parasiet-gastheer). Daarnaast kan onze analyse van antiknik- en stuurmechanismen bijdragen aan de ontwikkeling van nieuwe kunstmatige sondes, aangezien onze bevindingen bij kunnen dragen aan het minimaliseren van de dikte van de sonde en de verbetering van de sturingsmechanismen, wat beide bijdraagt aan verminderde weefselschade tijdens inserties. Onze bevindingen zijn met name interessant voor de ontwikkeling van slanke, stuurbare naalden voor minimaal-invasieve chirurgie. 


\section{Acknowledgements}

We all know that a PhD project is not a one-person endeavour. The research presented in this thesis could not have been done without the help and dedication of many people. Even though I might forget some, I would like to thank everyone I had the privilege to work with and those that contributed indirectly to my work by supporting me. You made these past four years an amazing experience.

To start, I would like to express my gratitude to my promotor Johan van Leeuwen. Thank you for giving me the opportunity to work at the Experimental Zoology Group (EZO) on the fascinating topic of probing insects. Your guidance, insightful suggestions, and advice on all aspects of my work are greatly appreciated. Thank you for believing in me and my capabilities. Next, I would like to thank my copromotor Sander Gussekloo, who patiently helped me taking my first awkward steps as a PhD candidate, fresh out of my MSc study, to becoming an independent researcher. I cannot express how amazing you are as a mentor. Thank you for always taking the time to address my questions, despite me barging in your office at any time of the day. I apologize for often being ever-so-slightly late to our weekly meetings, but you know how we 'Mediterraneans' are :). In addition to guiding my scientific development, we also shared our passion for climbing. Thank you for all the climbing advice, help with learning lead climbing, and opening up your home to me for the occasional stay over. Of course, my gratitude is also extended to your wife Irene, who made me feel welcome at your home and who sometimes had to change her evening plans because you were busy helping me with my PhD.

My sincere thanks also go to the collaborators from other universities, companies, and the national museum of natural history in Leiden (Naturalis Biodiversity Center). From the Technical University in Delft, I would like to thank Dimitra Dodou, Paul Breedveld, and Marta Scali. Your feedback, insights, and discussions on both the biological aspects of the project and the cutting-edge engineering developments greatly helped me during my work. Thank you for your contributions, it was a pleasure working with you. I should not forget the NWO WASP user committee. Jules and Maurits, thank you for your enthusiasm, support, and out of the box thinking during our progress meetings. Special thanks go to Prof. Dr Markus W. Hollmann for inviting me and Johan to the Amsterdam UMC and showing us some of the medical procedures that may benefit from novel surgical tools. I would also like to thank my collaborators and co-authors from Kiel University (Germany). Thank you Prof. Dr Stanislav Gorb, Dr Alexander Kovalev, and Dr Yoko Matsumura for the opportunity to conduct my experiments in Kiel. Your help with setting up the experimental setup, analysis, and discussions on the outcomes of my research 
are much appreciated. Special thanks go also to Frédérique Bakker from Naturalis Biodiversity Center, who allowed us to use the parasitic wasps from the museum collection in our measurements. I am also grateful to Dr Christian Schlepütz from the Paul Scherrer Institute (Switzerland) for his advice and help with the long measurements at the synchrotron facilities.

Many thanks go, of course, to the backbone of every research group: the lab technicians and the secretary. Thank you Karen, Remco, Henk, the new arrivals Frank and Kas, and Annemarie. I still remember the first year and a half (yes, no results to speak of for the first year and a half!) of testing, troubleshooting, and improving my first experimental setup, while at the same time trying to establish a breeding colony of parasitoid wasps. Karen, I am grateful for your help with taking care of the animals, making sure the colony did not collapse (it was fairly close a couple of times), and help with my measurements. Thank you Remco, for all the help with developing and troubleshooting the experimental setups, mounting and re-mounting all the cameras, and connecting everything so it worked as desired. And thank you Henk, for providing IT support, histological staining, the use of the numerous microscopes placed around the Zodiac building, and (do I dare mention it?) numerous external hard drives needed to store my data. Thank you Frank for your dedicated work on sectioning the probes of mosquitoes and parasitic wasps and thank you Kas for your taking care of the Naturalis Biodiversity Center collection. Dear Annemarie, I am grateful to you for taking care of me throughout both my MSc and PhD projects. You always say that we $\mathrm{PhD}$ candidates are like your children and you manage to make us feel like part of the big EZO family. Thank you for you kind words, support, and help when I tried to make sense of conference applications, administrative paperwork, dealing with the HR department, and making sure I had enough time to discuss my project with my supervisors, even though I sometimes requested additional meetings on a very short notice :).

The four years it officially takes to finish a $\mathrm{PhD}$ seem long and yet we ( $\mathrm{PhD}$ candidates) are always in need of more time. I am no different and right now, I would simply like more time to spend with all my friends and colleagues from EZO, neighbouring departments, and those I met outside the university setting.

Dear Julian and Pim, my paranymphs, thank you for all the support both in work and in personal matters. I could always count on you for sound advice and for cheering me up. I appreciate your genuine interest in science that sparked some nice discussions and your patience when I felt the need to complain. These thanks also extend to Annika, who always welcomed me with open arms. Julian and Annika, thanks for the nice evenings spent in Arnhem and I wish you all the best with your little Sophie. I have no doubt you will be awesome parents :). 
'Knock, knock... Cees, do you have a minute? I need some help with Matlab!' This is probably the most frequently enacted scene at EZO :D. Thank you Cees for your having the patience and always giving me (and many others) a helping hand when I struggled with analysis or programming.

EZO is a great place to work where everyone is glad to help each other out. Thank you Bart, Florian, Kees S., Martin, and Sander K. for the advice that steered me in my research and useful feedback during our weekly lunch meetings and journal clubs.

I was likewise fortunate to be surrounded by awesome colleagues that made work enjoyable and who made the coffee break conversations everything from bizarre to extremely funny. I will never forget the fun we had on lab-outings, WE-days, PhD-weekends, Friday afternoon drinks, 5th of May festivities, dinners, and many more occasions. Thanks you my colleagues at EZO (Andres, Antoine, Cees, Elsa, Henri, Gauthier, Lana, Maurijn, Mike, Myrthe, Pulkit, Sebastian, and Wouter), Cell Biology and Immunology Group (Adrià, Annelieke, Carmen, Christine, Esther, Éva D., Danilo, Joeri, Jules, Lieke, Mark, Marloes, Mirelle, Mojtaba, Olaf, Paulina, Sylvia, and Sem), Host-Microbe Interactomics Group (Bruno, Edoardo, Laura, Linda and Marcel, Marcela, Nadya, Raka, and Soumya), and at Berkeley CA, USA (Sofia). I was happy to be part of the 'core' climbing group together with Cees 'The Clamps' Voesenek :), Antoine 'The Smooth-talker' Cribellier :), Annelieke, and Sander G. who tried and sometimes succeeded in persuading others to join us in our fun weekly bouldering sessions where we put our elbows, knees, ankles (:/), and also faces on the line. Elsa, thank you for everything. We got to know each other in a course during my MSc studies. We often ended up in the same team for practicals and I like to think that is because we both shared the same interest and passion for experimental research. I still remember our friendly banter and how we complained about our projects to each other. Thank you for your support and I hope I did the same for you.

During my project, I had the opportunity to supervise and guide many BSc and MSc students. Thank you all! I learned a lot while working with you. Special thanks go to Bram and Noraly, who did outstanding work, which earned them co-authorship of parts of my thesis. Thank you for your dedication, long experimental hours trying to capture either the animals during probing or CT scanning innumerous specimens, and for your rigorous analysis of acquired data. It was a pleasure working with you.

I made many, many good friends in Wageningen and it would be too much to name them all. I would like to say special thanks to Aart-Jan, Bram, Corien, Dimitra M., Eva T., Fay, Ferdie, Iskra, Jose, Jimmy and Kathy, Julian Z., Kadi, Leah, Marlies, 
Meret, Michelle, Santos and Lucia, and Tim. Thanks for all the potluck dinners, nights out, salsa lessons, dance evenings, holiday trips (thank you Kadi for the lovely time we spent in Estonia :D), festivals, and many other things. Wageningen is a small town and many say that there is not much going on. However, with you in my life, there was always something interesting to do in Wageningen and I could relax and take my mind off the challenges I faced at work.

And last but not least, I would like to thank my family and friends. Najlepša hvala mami Nataši, očetu Daretu in sestri Nini. Hvala, da že od nekdaj verjamete vame in me podpirate v moji želji po znanju. Pomagali ste mi uresničiti svoje sanje, ne glede na to ali je bilo to glasbeno udejstvovanje, treniranje najljubšega športa, potovanje na drugo stran planeta, ali pa študij v tuji državi. Na Nizozemskem sem preživel dolgih sedem (skoraj osem) let. Hvala za obiske, izlete v bližnji in daljni okolici Wageningena, prijetne večere, redne telefonske in skajp pogovore in še in še. Ni bilo vedno lahko in brez vaše pomoči mi ne bi uspelo uspešno zaključiti najprej magisterija, sedaj pa še doktorata. Zelo vas imam rad in upam, da vam bom lahko kdaj povrnil vse, kar sem od vas prejel.

Hvala mojim prijateljem Aleksandru, Aljoši, Aneju, Borutu, Boštjanu, Črtu, Karlu, Klemenu in Urški, Rastku, Vikiju in Zoltanu, ki so me podpirali in spodbujali od doma. Čeprav so se naše poti oddaljile druga od druge, me veseli, da si še vedno redno vzamemo čas in se dobimo skupaj ob najrazličnejših priložnostih. Želim nam še veliko skupnih dogodivščin! :)

Posebna zahvala gre tudi Dr Gregorju Belušiču, za izkazano podporo, zaupanje in pomoč pri moji nadaljnji znanstveni karieri.

Thank you everyone for making my stay in Wageningen full of happy memories, laughter, excitement, interesting discussions and debates that helped me grow both as a scientist and as a person. My door is always open for anyone who decides to visit me wherever I end up living. Currently this is Slovenia, so not that far away :). 


\section{About the author}

\section{Biography}

Uroš Cerkvenik was born in Kranj, Slovenia, on 7th of August 1988. After finishing the gymnasium programme at the Srečko Kosovel secondary school in his home town Sežana, he enrolled in the Biology BSc programme at the Biotechnical Faculty of the University of Ljubljana (Slovenia). During his BSc studies, he became interested in animal sensory physiology and biomimetics. As no MSc study programmes focus on both of these research fields in Slovenia, Uroš looked for a suit-

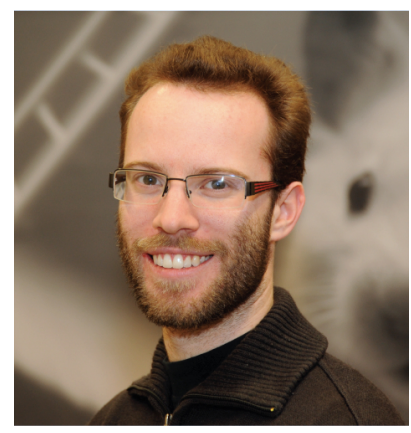
able study abroad. He discovered the Experimental Zoology Group (EZO) at Wageningen University \& Research (WUR) in the Netherlands and was immediately intrigued by the field of biomechanics. Uroš acquired a grant from the Slovenian Public Fund for Development of Human Resources and Scholarships and enrolled in the Biology MSc programme at WUR.

In the Netherlands, Uroš expanded his knowledge on animal sensory systems. He did an internship at the Radboud University in Nijmegen (the Netherlands), studying the tuning of neurons in the primary visual cortex in mice under the supervision of Prof. Dr Richard J.A. van Wezel at the Visual Neuroscience Research Group. Uroš combined his interests in sensory biology and biomechanics in his MSc thesis project by investigating the hearing system in field crickets under the supervision of Dr Martin Lankheet (EZO). For his MSc thesis project, Uroš was awarded the 2015 thesis prize by the Wageningen Alumni Network (WUF-KLV). His thesis was later published in a revised form as a peer-reviewed paper in the $J$. Royal Soc. Interface.

Uroš graduated from his MSc cum laude and soon after started his PhD project at EZO. The aim of this project, which was funded by the Netherlands Organization for Scientific Research Division Applied and Engineering Sciences (NWO AES), was to investigate the probing biomechanics of parasitic wasps in relation to the development of steerable needles for minimally invasive surgery. Under the supervision of Prof. Dr Johan L. van Leeuwen and Dr Sander W.S. Gussekloo, Uroš studied key aspects of insect probing, including the kinematics of probe insertion, probe material properties, and its actuation system. Uroš worked closely with the PhD Candidate Marta Scali, Dr Dimitra Dodou, and Prof. Dr. Paul Breedveld from the Delft University of Technology (Netherlands), who developed a new prototype of 
a steerable needle based on the probe of the wasps. Additionally, Uroš collaborated with researchers from Kiel University (Germany) and Paul Scherrer Institute (Switzerland), where he conducted part of his experiments. Uroš published his research in journals including PNAS and Biol. Rev., and also received the WIAS publication prize 2017. The findings of his PhD research can be found in this thesis entitled Biomechanics of the wasp ovipositors: Probing for answers. 


\section{List of publications}

\section{Peer-reviewed scientific publications}

1. Cerkvenik U, Dodou D, van Leeuwen JL, \& Gussekloo SWS (2018) Functional principles of steerable multi-element probes in insects. Biological Reviews of the Cambridge Philosophical Society. doi: 10.1111/brv.12467

2. Cerkvenik U, van de Straat B, Gussekloo SWS, \& van Leeuwen JL (2017) Mechanisms of ovipositor insertion and steering of a parasitic wasp. Proceedings of the National Academy of Sciences of the United States of America 114, E7822-E7831. doi: 10.1073/pnas.1706162114

3. Lankheet MJ, Cerkvenik U, Larsen ON, \& van Leeuwen JL (2017) Frequency tuning and directional sensitivity of tympanal vibrations in the field cricket Gryllus bimaculatus. Journal of the Royal Society Interface 14, 20170035. doi: $10.1098 /$ rsif.2017.0035

\section{Conference and symposia proceedings}

1. Cerkvenik U, van Leeuwen JL, \& Gussekloo SWS. Ovipositor stiffness gradients facilitate its steering. Annual meeting of the Society of Experimental Biology (SEB). July $3^{\text {rd }}-7^{\text {th }} 2018$, Florence, Italy.

2. Cerkvenik U, van de Straat B, Gussekloo SWS, \& van Leeuwen JL. How parasitic wasps steer ovipositors and avoid buckling during probing. Annual meeting of the Society for Integrative and Comparative Biology (SICB). January $3^{\text {rd }}$ $7^{\text {th }} 2018$, San Francisco, United States of America.

3. van Meer NMME, Cerkvenik U, \& Gussekloo SWS. High resolution 3D imaging of a parasitic wasp ovipositor base shows its role in probing. Annual meeting of the Society of Experimental Biology (SEB). July $3^{\text {rd }}-6^{\text {th }} 2017$, Gothenburg, Sweden.

4. Cerkvenik U, van de Straat B, Gussekloo SWS, \& van Leeuwen JL. How parasitic wasps explore the unknown with their steerable ovipositors. Annual meeting of the Society of Experimental Biology (SEB). July $4^{\text {th }}-7^{\text {th }} 2016$, Brighton, United Kingdom.

5. Cerkvenik U, van de Straat B, Gussekloo SWS, \& van Leeuwen JL. Exploration capabilities of parasitic wasp ovipositors, Joint conference of the international Society for Medical Innovation and Technology (iSMIT) and Design of Medical Devices Europe (DMD-EU). October $5^{\text {th }}-8^{\text {th }} 2016$, Delft, Netherlands. 
6. Gussekloo SWS, Heinen R, \& Cerkvenik U. Adaptations to substrate properties in the ovipositors of parasitic wasps (Ichneumonoidea). Annual meeting of the Society for Integrative and Comparative Biology (SICB). January $3^{\text {rd }}-7^{\text {th }} 2016$, Portland, United States of America.

7. CeRKVEnik U, Gussekloo SWS, \& van LeeuWen JL. Biomechanics of ovipositor insertion into artificial homogeneous substrate: Towards the quantification of the insertion and steering mechanisms. Annual meeting of the Society of Experimental Biology (SEB). June $29^{\text {th }}-J u l y 3^{\text {rd }} 2015$, Prague, Czech Republic.

8. Cerkvenik U, Gussekloo SWS, \& van Leeuwen JL. Parasitic wasps as inspiration for ultra-thin steerable needles. Design of Medical Devices Europe (DMDEU) Conference. October $22^{\text {nd }}-24^{\text {th }} 2014$, Delft, Netherlands. 


\section{Overview of completed training activities}

\begin{tabular}{lcc}
\hline Activity & Year & ECTS \\
\hline The basic package & & 3 \\
\hline WIAS Introduction Course & 2014 & \\
Ethics and Philosophy in Life Sciences & 2014 \\
Scientific exposure & & $\mathbf{1 4}$ \\
\hline
\end{tabular}

International conferences

Design of Medical Devices Europe (DMD-EU)

Delft, Netherlands

Society of Experimental Biology (SEB)

Prague, Czech Republic

2016

Brighton, United Kingdom

international Society for Medical Innovation

and Technology (iSMIT) and

Design of Medical Devices (DMD-EU)

Delft, Netherlands

Society of Integrative and Comparative Biology (SICB)

San Francisco, CA, United States of America

Society of Experimental Biology (SEB)

Florence, Italy

Seminars and workshops

WIAS Science Day

WGS Carousel

Medical Delta MedTechWest

instruments for Minimally Invasive Procedures (iMIT)

consortium meetings

Poster presentations

Society of Experimental Biology (SEB)

Prague, Czech Republic

2015

Design of Medical Devices

Oral presentations

Design of Medical Devices Europe (DMD-EU)

Delft, Netherlands

Society of Experimental Biology (SEB)

Brighton, United Kingdom

international Society for Medical Innovation

and Technology (iSMIT) and

Design of Medical Devices (DMD-EU)

Delft, Netherlands

Society of Integrative and Comparative Biology (SICB)

San Francisco, CA, United States of America

Society of Experimental Biology (SEB)

Florence, Italy 
In-depth studies

$8^{\text {th }}$ Zürich Summer School on Biomedical Imaging

Zürich, Switzerland

2014

WIAS Course 'Statistics for the Life Sciences'

2015

Linear Modelling (including FEM)

2015

Statutory courses

Radiation protection level 5B

Leiden, Netherlands

Professional skills support courses

WIAS Course: High-Impact Writing in Science

WIAS Course: Survival Guide to Peer Review

WIAS Course: Scientific Publishing

WIAS Course: Reviewing a Scientific Paper

2016

Educational Staff Development Course:

Supervising BSc and MSc thesis students

WIAS Course:

The Final Touch: Writing the General Introduction and Discussion

Research skills training

PhD Research Proposal

MATLAB Programming Techniques

2014

Didactic skills training

Supervision of $3 \mathrm{BSc}$ theses

$2015,2016,2018$

Supervision of $6 \mathrm{MSc}$ theses/internships

2014-2018

Total

One ECTS credit equals a study load of approximately 28 hours. 

The research described in this thesis was financially supported by the Netherlands Organization for Scientific Research Division Applied and Engineering Sciences (NWO TTW/NWO AES), grant number 12712.

Financial support from the Experimental Zoology Group of Wageningen University for printing this thesis is gratefully acknowledged.

Design of the cover and the artwork accompanying chapter title-pages:

Gaja Hanzel, mag. graf. inž. (MFA)|Malina Design|www.malinadesign.si 



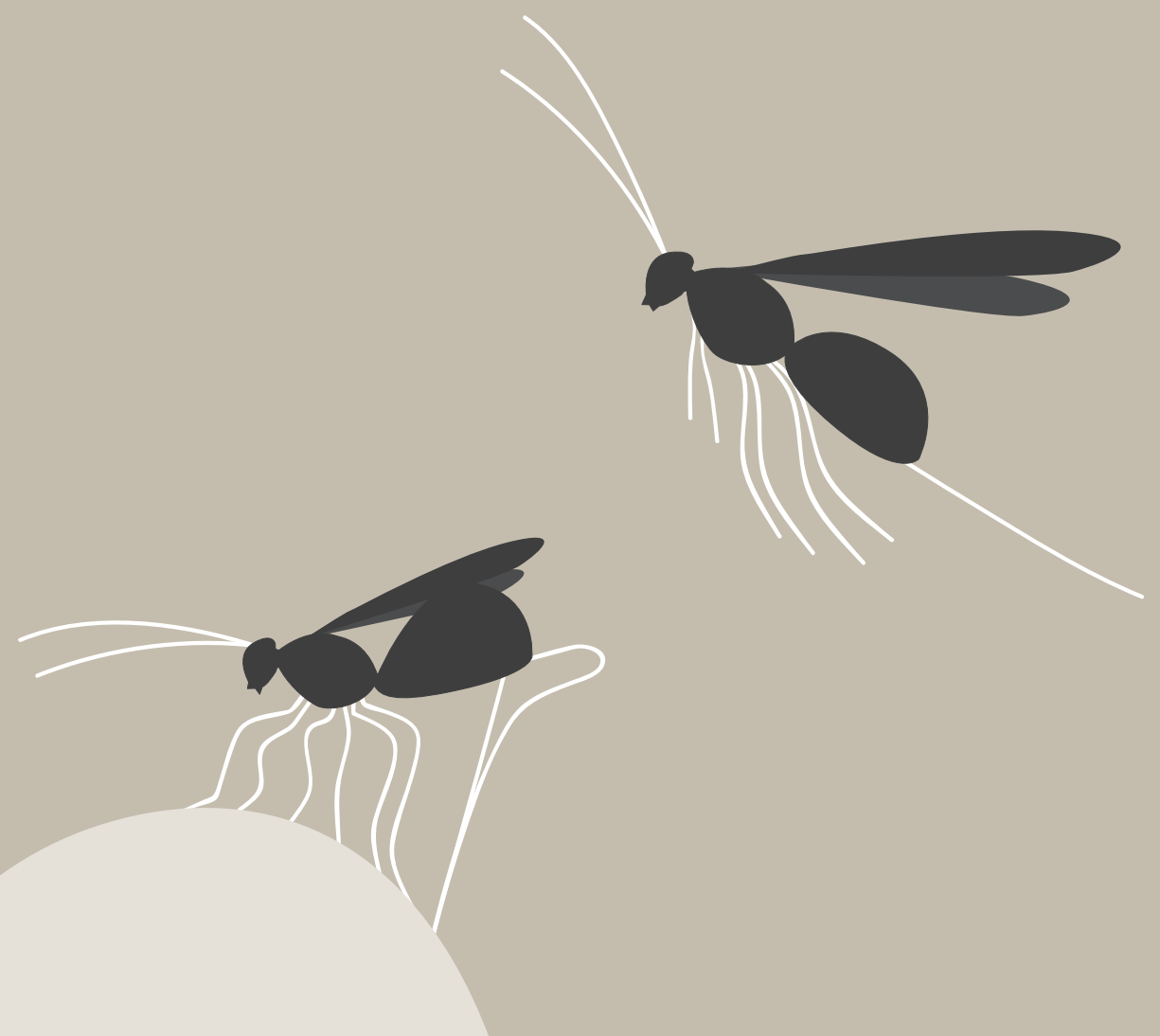

